\title{
Norepinephrine ignites local hotspots of neuronal excitation: How arousal amplifies selectivity in perception and
} memory

\author{
Mara Mather \\ Davis School of Gerontology, University of Southern California, Los Angeles, \\ CA 90089-0191 \\ mara.mather@usc.edu

\section{David Clewett} \\ Neuroscience Graduate Program, University of Southern California, \\ Los Angeles, CA 90089-2520 \\ clewett@usc.edu \\ Michiko Sakaki \\ School of Psychology and Clinical Language Sciences, University of Reading, \\ Reading RGX 7BE, United Kingdom; and Kochi University of Technology, \\ Kami, 782-0003, Japan \\ m.sakaki@reading.ac.uk
}

Carolyn W. Harley

Professor Emeritus, Memorial University of Newfoundland, St. John's, NL A1C 5S7, Canada

charley@play.psych.mun.ca

\begin{abstract}
Emotional arousal enhances perception and memory of high-priority information but impairs processing of other information. Here, we propose that, under arousal, local glutamate levels signal the current strength of a representation and interact with norepinephrine (NE) to enhance high priority representations and out-compete or suppress lower priority representations. In our "glutamate amplifies noradrenergic effects" (GANE) model, high glutamate at the site of prioritized representations increases local NE release from the locus coeruleus (LC) to generate "NE hotspots." At these NE hotspots, local glutamate and NE release are mutually enhancing and amplify activation of prioritized representations. In contrast, arousal-induced LC activity inhibits less active representations via two mechanisms: 1) Where there are hotspots, lateral inhibition is amplified; 2) Where no hotspots emerge, NE levels are only high enough to activate low-threshold inhibitory adrenoreceptors. Thus, LC activation promotes a few hotspots of excitation in the context of widespread suppression, enhancing high priority representations while suppressing the rest. Hotspots also help synchronize oscillations across neural ensembles transmitting high-priority information. Furthermore, brain structures that detect stimulus priority interact with phasic NE release to preferentially route such information through large-scale functional brain networks. A surge of NE before, during, or after encoding enhances synaptic plasticity at NE hotspots, triggering local protein synthesis processes that enhance selective memory consolidation. Together, these noradrenergic mechanisms promote selective attention and memory under arousal. GANE not only reconciles apparently contradictory findings in the emotion-cognition literature but also extends previous influential theories of LC neuromodulation by proposing specific mechanisms for how LC-NE activity increases neural gain.
\end{abstract}

Keywords: arousal; attention; emotion; locus coeruleus; long-term consolidation; memory; norepinephrine; perception

\section{Introduction}

When jolted by a rough skydiving landing, psychologist James Easterbrook observed that his sense of space and time shrank and slowly re-expanded (Easterbrook 1982). This sparked his curiosity about how arousal influences attention. Later he published a review article in which he argued that under arousal, people rely more on central or immediately relevant information and less on peripheral information (Easterbrook 1959). Since his seminal paper, researchers have accumulated many more observations that arousal evoked by emotional events enhances some aspects of perception and memory but impairs others (for reviews, see Mather \& Sutherland 2011; Reisberg \& Heuer 2004). For example, victims of a crime tend to remember the weapon vividly but forget the perpetrator's face (Steblay 1992). People also pay attention 
to emotional information at the expense of neutral information (Dolcos \& McCarthy 2006; Knight et al. 2007). These examples fit with Easterbrook's formulation that arousal impairs attention to peripheral information. But arousing stimuli can sometimes enhance memory of peripheral neutral information (Kensinger et al. 2007; Knight \& Mather 2009). Thus, although it is clear that arousal shapes attention and memory, knowing that something is neutral or spatially peripheral is not enough to predict how it will fare under emotional conditions.

So, then, how does arousal influence the brain's selection of features to highlight versus suppress? An initial answer to this puzzle was provided by the arousal-biased competition (ABC) model, which posits that arousal does not have fixed rules about which types of stimuli to enhance or suppress. Instead, arousal amplifies the stakes of ongoing selection processes, leading to "winner-take-more" and "loser-take-less" effects in perception and memory (Mather \& Sutherland 2011). The ABC model builds on biased competition models proposing that stimuli must compete for limited mental resources (Beck \& Kastner 2009; Desimone \& Duncan 1995; Duncan 2006). As conceptualized by Desimone and Duncan (1995), both bottom-up and top-down neural mechanisms help resolve competition.

David Clewet, who received his Ph.D. in Neuroscience, has been the recipient of two Endowed Fellowships from the University of Southern California. His research focuses on the role of the noradrenergic system in mediating the influence of arousal on memory and healthy cognitive aging. In 2014, he was recognized as the program's Neuroscience Student of the Year.

Carolyn Harley, Professor Emeritus in Psychology and Neuroscience at Memorial University of Newfoundland, is the author of more than 95 publications in learning and memory and brain plasticity with a special focus on norepinephrine and the locus coeruleus. She has served as President of the Canadian Society for Brain, Behavior and Cognitive Science. Her ongoing research program is concerned with behavioral functions of noradrenergic locus coeruleus activation and is funded by the Natural Sciences and Engineering Research Council of Canada.

Mara Mather, Professor of Gerontology and Psychology at the University of Southern California, conducts research focusing on emotion-cognition interactions with a particular focus on how emotional arousal shapes perception and memory. She has received the Distinguished Scientific Award for Early Career Contribution to Psychology from the American Psychological Association, an Alexander von Humboldt fellowship, and a National Institutes of Health K02 Career Development award.

Michiko SaKaki is a Senior Research Fellow in Psychology at the University of Reading, where she examines the effects of emotion on cognitive processing across younger and older adults. Her research has been supported by the European Commission and the British Academy, and in 2014 she was awarded the faculty research output prize by the Faculty of Life Sciences, University of Reading.
Bottom-up processes are largely automatic, determined by the perceptual properties of a stimulus, and do not depend on top-down attention or task demands. For example, stimuli that contrast with their surroundings, such as a bright light in a dark room, engage attention automatically even if they are currently goal irrelevant (Itti \& Koch 2000; Parkhurst et al. 2002; Reynolds \& Desimone 2003). Top-down goals can also bias competition in favor of particular stimuli that otherwise would not stand out. Although not included in the original biased competition models, past history with particular stimuli is also a source of selection bias (Awh et al. 2012; Hutchinson \& Turk-Browne 2012). For example, one's name or a novel stimulus tends to engage attention (Moray 1959; Reicher et al. 1976). In addition, faces, text, and emotionally salient stimuli all grab attention (e.g., Cerf et al. 2009; Knight et al. 2007; MacKay et al. 2004; Niu et al. 2012).

A core aspect of most current theories of visual attention is that these different signals are integrated into maps of the environment that indicate the priority or salience of stimuli across different locations (Itti \& Koch 2000; Soltani \& Koch 2010; Treisman 1998). Regions in frontoparietal cortex integrating sensory and top-down signals help represent such priority maps (Ptak 2012). Moreover, having both feedforward and feedback connections between sensory regions and cortical priority maps enables distributed representations of prioritized information to modulate their own processing (e.g., lower-level visual features) even further (Klink et al. 2014; Ptak 2012; Serences \& Yantis 2007; Soltani \& Koch 2010). Thus, priority signals are self-biasing to enhance efficient information processing in the brain.

In the ABC model, arousal further biases mental processing to favor high- over low-priority representations, regardless of whether initial priority is determined by bottom-up salience, emotional salience, or top-down goals. Thus, because spatially peripheral information is usually lower priority than central information, arousal usually impairs memory for it (Steblay 1992; Waring \& Kensinger 2011). Yet, when peripheral information is perceptually salient or goal relevant, arousal instead enhances memory for it (e.g., Kensinger et al. 2007, Experiment 4). But the ABC model does not tackle how this works in the brain. Previous brain-based models of emotion and cognition also do not account for the dual role of arousal. Most models posit that the amygdala enhances perception and memory consolidation of emotionally salient stimuli, but fail to address how arousal sometimes enhances and sometimes impairs information processing.

In this article we propose the glutamate amplifies noradrenergic effects (GANE) model, in which arousal amplifies the activation difference between high- and low-priority representations via local synaptic self-regulation of the locus coeruleus-norepinephrine (LC-NE) system. According to the GANE model, hearing an alarming sound or seeing something exciting leads to a surge in $\mathrm{NE}$ release, which, in turn, enhances activity of neurons transmitting high-priority mental representations and suppresses activity of neurons transmitting lower-priority mental representations. As already outlined, priority is determined by top-down goals, bottom-up factors, and high-level stimulus features (Beck \& Kastner 2009; Desimone \& Duncan 1995; Fecteau \& Munoz 2006).

According to the GANE model, the brain's primary excitatory neurotransmitter, glutamate, signals priority. 
Under arousal, elevated glutamate associated with highly active neural representations stimulates greater $\mathrm{NE}$ release, which then further increases glutamate via positive feedback loops. Thus, in these local "NE hotspots," glutamate signals are amplified. At the same time, wherever $\mathrm{NE}$ is released and fails to ignite a local hotspot, inhibitory adrenoreceptors with lower thresholds of activation suppress activity. Higher NE concentration at hotspots also enhances delivery of energy resources to the site of active cognition, synchronizes brain oscillations, and modulates activity in large-scale functional networks. Thus, under arousal, local NE hotspots contrast with widespread NE suppression to amplify priority effects in perception and memory, regardless of how priority was instantiated.

\section{Arousal-biased competition in perception and memory}

We start by reviewing recent findings supporting Mather and Sutherland's (2011) ABC model and its novel predictions. Next, we turn to the question of how these arousal effects operate in the brain. A fundamental challenge in understanding how arousal influences cognition is that it sometimes enhances and sometimes impairs information processing. Although most emotion research focuses on how processing of emotional stimuli is enhanced compared with neutral stimuli, emotional arousal can also influence processing of neutral stimuli, and across studies, opposing effects are often seen. How can emotionally salient stimuli sometimes enhance memory for what just happened, but other times impair it? When do arousing stimuli enhance perception and when do they impair perception of subsequent stimuli? Many studies report that emotion increases selectivity (for reviews, see Levine \& Edelstein 2009; Mather \& Sutherland 2011; Murray et al. 2013), but how do we predict what gets selected?

\subsection{Arousal enhances perception of salient stimuli, but impairs perception of inconspicuous stimuli}

In previous research on how arousal influences subsequent perception, two types of findings were hard to reconcile. First, arousing stimuli impair perception of subsequent stimuli. For example, people preferentially perceive arousing stimuli (e.g., Anderson 2005; Keil \& Ihssen 2004) but fail to perceive or encode neutral stimuli close to arousing stimuli either in time (e.g., embedded in a rapid series of images after an arousing image) (Smith et al. 2006) or in space (Kensinger et al. 2007; Tooley et al. 1987). Second, hearing or seeing an arousing stimulus enhances visual perception of a subsequent Gabor patch (Lee et al. 2014a; Padmala \& Pessoa 2008; Phelps et al. 2006).

How can we explain both the enhancing and impairing effects of arousing stimuli on perception of stimuli that appear close in time or space? Initial evidence supports the $\mathrm{ABC}$ hypothesis that inducing arousal should have two opposing effects on perception: Arousal should enhance processing of high-priority (more salient) stimuli but impair processing of lower-priority (less salient) stimuli. When asked to report as many letters as they could from a briefly flashed array (Fig. 1), participants reported more of the high-salience letters and fewer of the low-salience letters after hearing an arousing emotionally negative sound than after hearing a neutral sound (Sutherland \& Mather 2012). Similar results were obtained when arousal was induced by emotionally positive sounds (Sutherland \& Mather, under review). These results indicate that arousal makes salient stimuli stand out more than they would otherwise.

The ABC model also explains the enhanced processing of emotional stimuli, the focus of most previous theoretical accounts (e.g., Kensinger 2004; LaBar \& Cabeza 2006; Mather 2007; Murty et al. 2010; Phelps 2004). People tend to prioritize emotional stimuli due to top-down goals (e.g., increasing pleasure and avoiding pain), their emotional saliency (e.g., associations with reward/punishment), and/or bottom-up salience (e.g., a gunshot is loud as well as a threat to safety [Markovic et al. 2014]). Thus, arousing stimuli should dominate competition for representation at their particular spatiotemporal position (Wang et al. 2012).

If the arousing stimulus appears in the exact same location as a neutral stimulus presented less than a second later, it will impair perception of that neutral stimulus, an effect known as emotion-induced blindness (Kennedy \& Most 2012; Most et al. 2005). On the other hand, arousing stimuli tend to enhance the dominance of high-priority stimuli that are nearby but not competing for the same
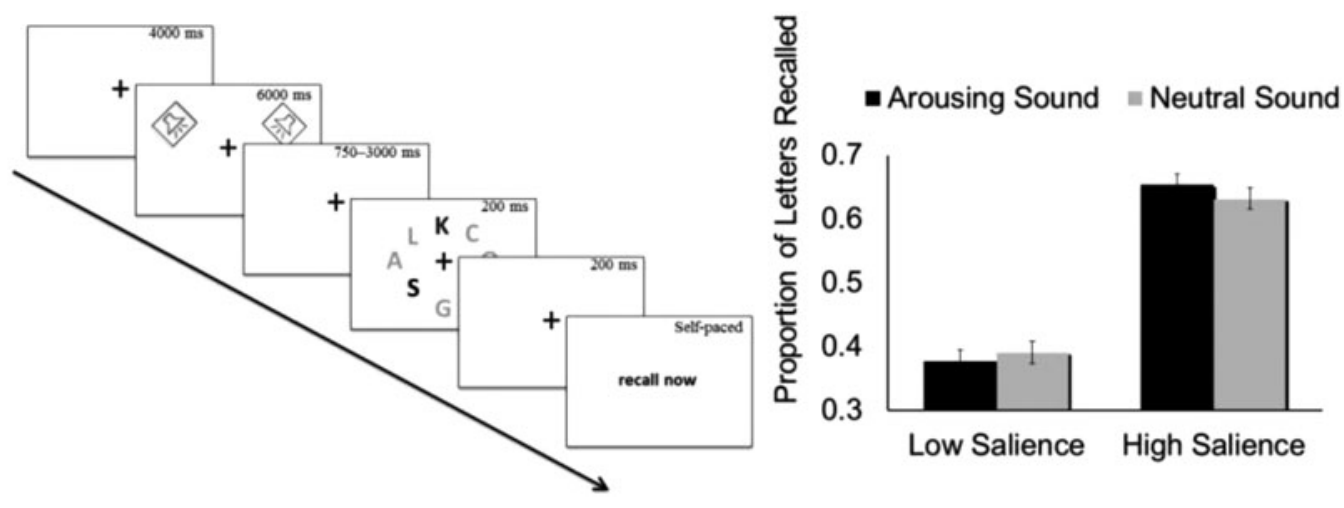

Figure 1. Participants heard an arousing or neutral sound before a letter array was flashed briefly. They then reported as many of the letters as they could. Some of the letters were shown in dark gray (high contrast and, therefore, salient) and some in light gray (low contrast and less salient). Participants reported a greater proportion of the salient letters than the nonsalient letters, but this advantage for salient letters was significantly greater on arousing trials than on neutral trials, and the disadvantage for the nonsalient letters was significantly greater on arousing than on neutral trials (Sutherland \& Mather 2012). 
Mather et al.: Norepinephrine ignites local hotspots of neuronal excitation

spatiotemporal spot. An emotionally salient word that impairs perception of a subsequent target word flashed in the same location 50 or 500 ms later can instead enhance perception of a target word flashed 1,000 ms later (Bocanegra \& Zeelenberg 2009), because after the longer interval, the priority of the target word is no longer overshadowed by the emotionally salient word.

\subsection{Arousal enhances perceptual learning about salient stimuli but impairs learning about nonsalient stimuli}

Interspersing emotional or neutral pictures with a visual search task had opposite effects on perceptual learning of salient and nonsalient targets (Lee et al. 2012). In this study, the targets were always the same, but in one condition they were salient because they differed from the distractors, and in the other condition they were not salient because they were quite similar to distractors. Emotional images enhanced perceptual learning about the salient target lines but impaired learning of nonsalient targets (Fig. 2). Thus, whether arousal enhanced or impaired learning depended on the target's salience.

\subsection{How arousal modulates neural representations depends on salience}

A recent study took advantage of the fact that faces and scenes activate distinct representational regions in the brain to test the $\mathrm{ABC}$ hypothesis that arousal increases brain activation associated with processing of salient stimuli, whereas it decreases brain activation associated with processing of less salient stimuli (Lee et al. 2014b). On each trial, one yellow-framed face and one scene image appeared briefly side-by-side and then a dot appeared in the former location of one of the images (Fig. 3A). The participants' task was to indicate the side on which the dot appeared. Participants responded fastest to dots that appeared behind the salient faces on trials preceded by a tone conditioned to predict shock and thereby induce arousal. In a follow-up functional magnetic resonance imaging (fMRI) study, there was an arousal $\times$ saliency interaction in visual category-specific brain regions, such that arousal enhanced brain activation in the region processing the salient stimulus (i.e., fusiform face area) but suppressed brain activation in the region processing the nonsalient stimulus (i.e., parahippocampal place area) (Fig. 3B) (Lee et al. 2014b).

\subsection{Arousal enhances or impairs memory consolidation of representations depending on their priority}

So far, we have focused on how arousal enhances processing of subsequent inputs; however, arousal should have similar effects on mental representations currently active at the moment arousal is induced. Previous research has indicated that arousal induced after initial encoding sometimes impairs and sometimes enhances memory of preceding information (Knight \& Mather 2009). The critical ABC hypothesis is that experimental manipulation of priority of information should alter the effect of subsequent arousal on memory consolidation.

In the first study testing this hypothesis, participants viewed lists of objects one object at a time, with one perceptual oddball in each list (Fig. 4) (Sakaki et al. 2014a). The oddball was either emotionally salient or neutral. Some participants were asked to recall the name of the oddball picture as soon as the list presentation ended. In this condition, the object shown just before the oddball (e.g., the cabbage in Fig. 4) was low priority. Other participants were asked to recall the name of the object shown just before the oddball (oddball-minus-1 object). Thus, in this condition, the oddball-minus-1 object (e.g., the cabbage) was high priority. After a series of lists, memory for details of all oddball-minus-1 objects was tested. As predicted, positively or negatively emotionally salient oddball pictures enhanced memory for prioritized oddball-minus-1 objects and impaired memory for nonprioritized oddball-minus-1 objects.

Although the brain mechanisms underlying this priority $\times$ arousal interaction in memory have yet to be tested, fMRI evidence indicates that arousal enhances activity in regions processing a high-priority stimulus. For example, pairing shock with certain high-priority (i.e., standalone) neutral scenes enhances successful encoding-related activity in the parahippocampal place area (PPA), the brain region specialized to process scene information (Schwarze et al. 2012). Thus, arousal-induced enhancement of brain
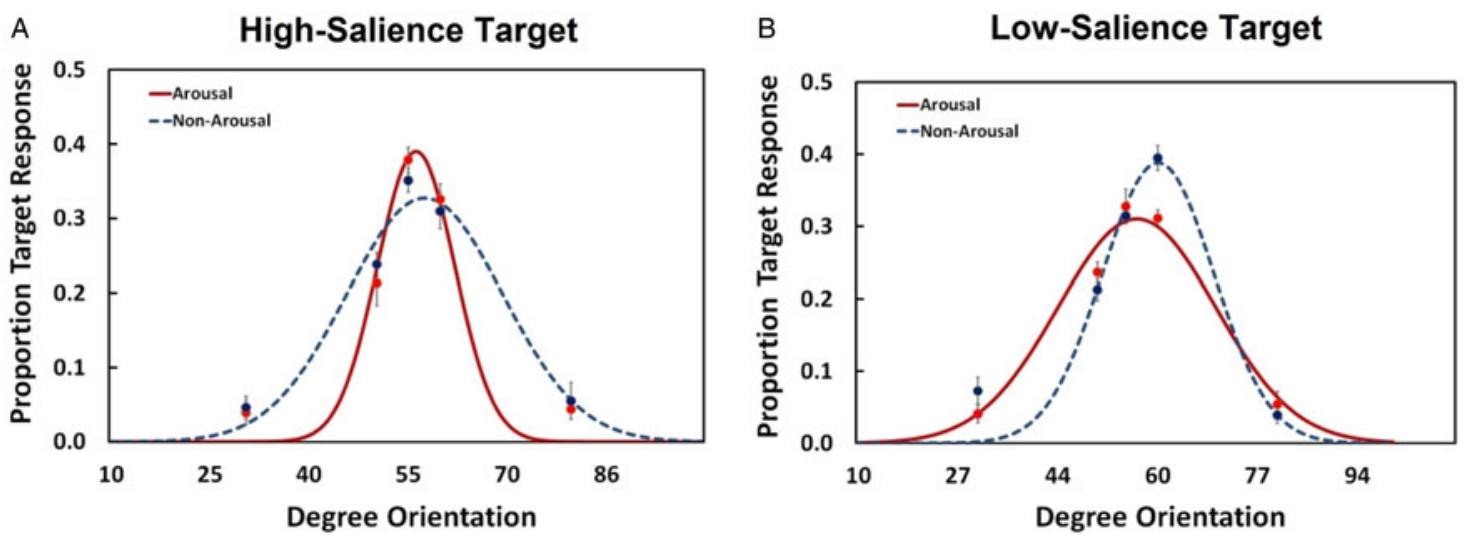

Figure 2. Estimated tuning curves for averaged "target" responses as a function of emotion in the high-salience condition (A) and lowsalience condition (B). In the high-salience condition, having interspersed emotional pictures enhanced perceptual learning of the exact tilt of the target $\left(55^{\circ}\right)$, whereas in the low-salience condition, emotion impaired learning of the exact tilt of the same target. Figure adapted from Lee et al. (2012). 
A Dot Probe Task
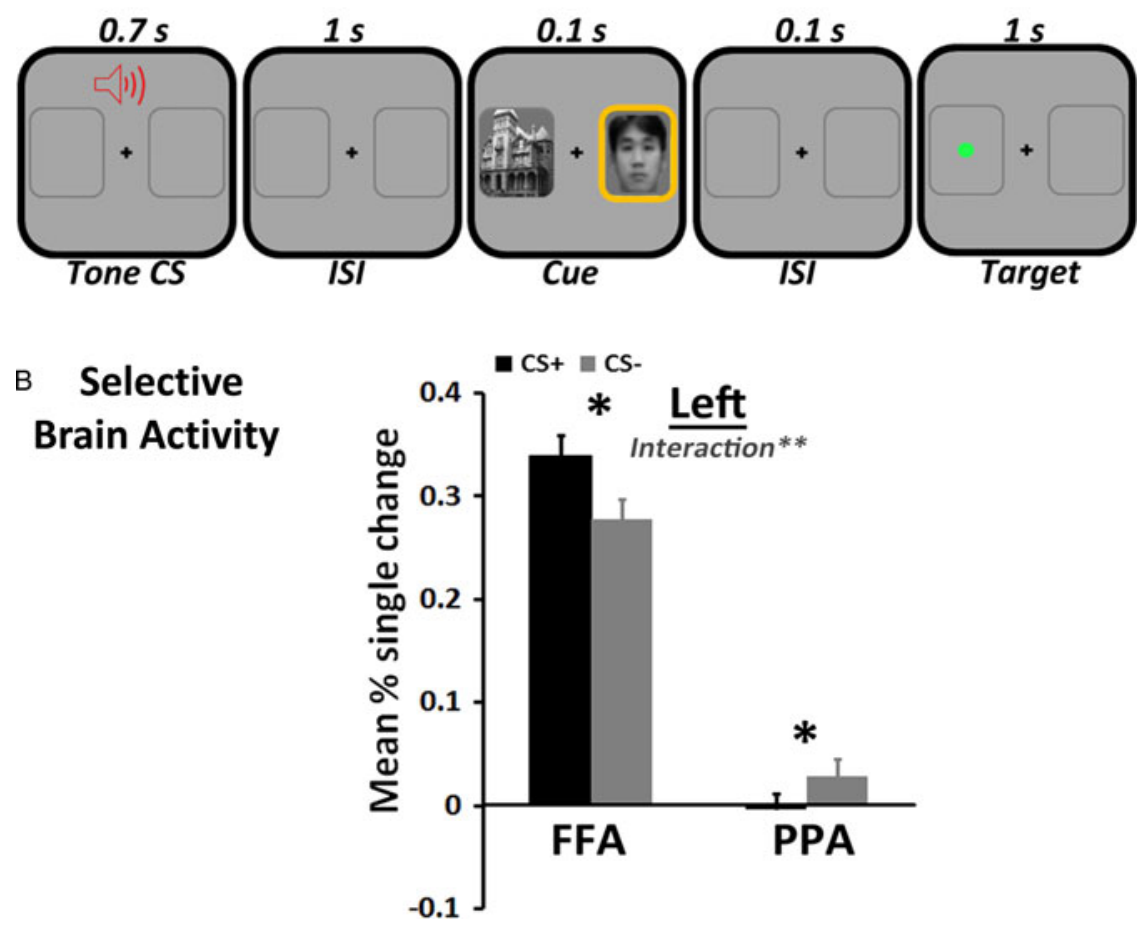

Figure 3. In the functional magnetic resonance imaging study by Lee et al. (2014b), tones conditioned to predict shock (CS + tones) played before the display of a salient face, and a less salient scene (A) increased activity in the left fusiform face area (FFA) associated with face processing, while decreasing activity in the left parahippocampal place area (PPA) associated with scene processing, compared with tones conditioned not to predict shock $($ CS- tones $)(\mathbf{B}) .{ }^{*} \mathrm{p}<0.05,{ }^{* * *} \mathrm{p}<0.005$. CS $=$ conditioned stimulus; ISI $=$ interstimulus interval.

activity processing prioritized information not only occurs during perception (e.g., Lee et al. 2014b), but also predicts memory for such items.

\subsection{Summary}

Mather and Sutherland's (2011) ABC model accounts for both the enhancement and impairment effects of arousal on neutral stimuli across a wide variety of experimental contexts. It makes novel predictions: (1) Arousal before exposure to stimuli should amplify the effects of salience on perception and memory encoding; and (2) Arousal shortly after encoding information should amplify the effects of its goal relevance on memory consolidation. Both effects result from arousal's differential modulation of representations depending on priority. Other models also highlight the importance of interactions between arousal, attention, and goals (Kaplan et al. 2012; Levine \& Edelstein 2009; Montagrin et al. 2013; Talmi 2013). However, so far there has been no account of how arousal amplifies the effects of priority in the brain.

\section{Current brain-based models of arousal's modulatory effects}

Before we present our account of how arousal can modulate neural representations differently depending on their priority, we outline how existing brain-based models of arousal and cognition fail to adequately address how arousal has opposite effects depending on representational priority (see Table 1 for an overview).

\subsection{Modular vs. "multiple waves" of emotion enhancement in perception}

Noticing things like snakes and guns can increase the odds of survival. Consistent with this adaptive importance, emotionally salient stimuli are often detected more rapidly than neutral stimuli (Leclerc \& Kensinger 2008; Mather \& Knight 2006; Öhman et al. 2001). Explaining the privileged status of emotional stimuli has been the focus of brain models of emotion perception. One common assumption is that the evolutionary value of noticing emotional stimuli led to a specialized emotion module or pathway to evaluate emotional salience (Tamietto \& de Gelder 2010). For example, in their multiple attention gain control (MAGiC) model, Pourtois et al. (2013) argue that emotional salience shapes perception via amplification mechanisms independent of other attentional processes. In the MAGiC model, the amygdala and other modulatory brain regions amplify neural responses to emotional relative to neutral stimuli along sensory pathways. The model also posits that these modulations occur parallel to and sometimes in competition with signals from bottom-up 
Mather et al.: Norepinephrine ignites local hotspots of neuronal excitation
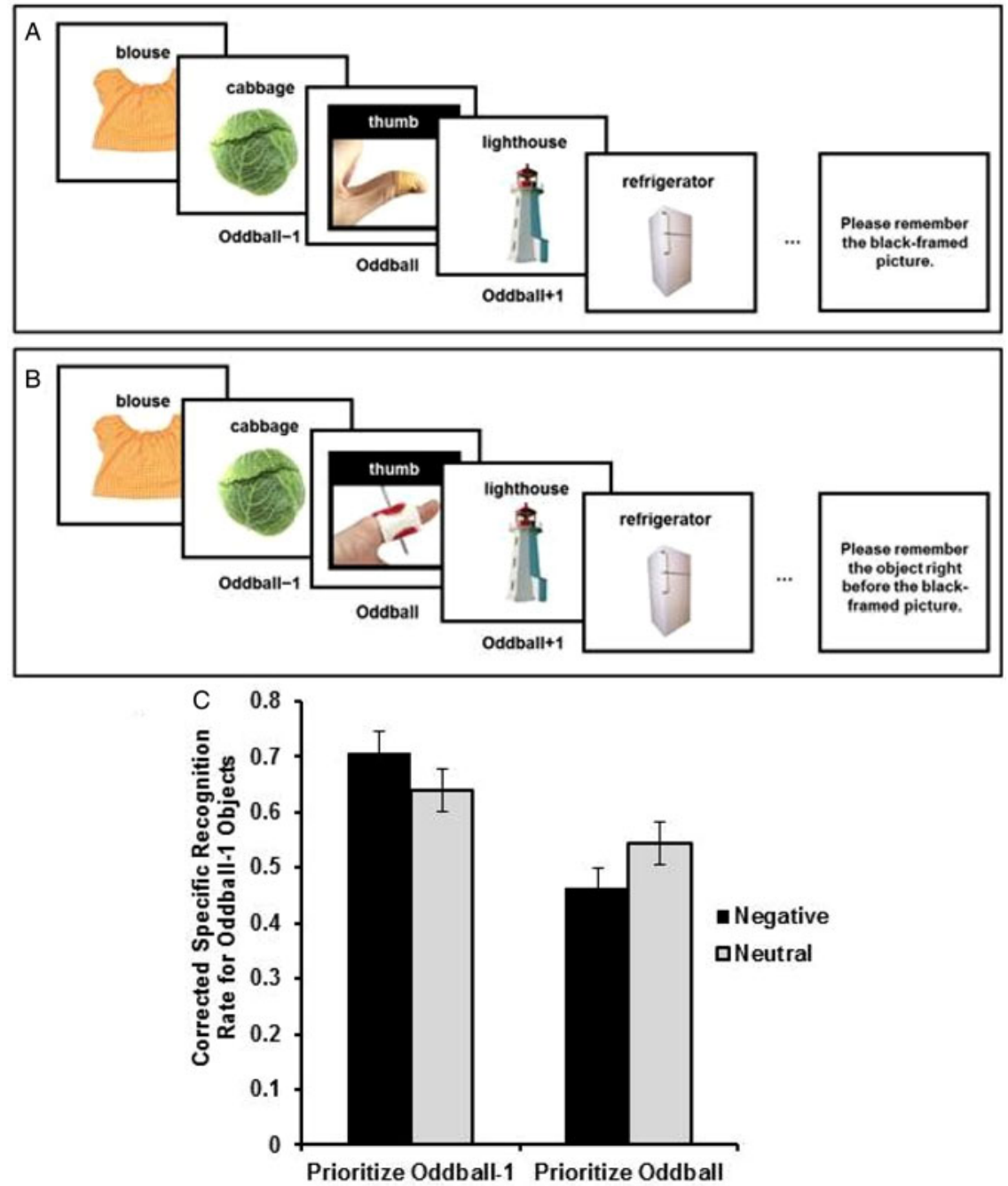

Figure 4. Schematic representations of a neutral trial in the prioritize-oddball condition $(\mathbf{A})$ and a negative trial in the prioritize-oddballminus-1 condition (B). Memory performance for oddball-minus-1 objects differed as a function of their priority and the valence of oddball pictures $(\mathbf{C})$. Oddball pictures depicted here were obtained from iStockPhoto for illustration purposes and differ from those used in the experiments. Figures from Sakaki et al. (2014a).

(exogenous) and top-down (endogenous) attentional control systems (see also Vuilleumier 2005b).

In contrast, Pessoa and Adolphs (2010) argue against a modular approach to emotion enhancement in perception. In their multiple waves model, affectively and motivationally significant visual stimuli rapidly engage multiple brain sites, including the amygdala, orbitofrontal cortex, anterior insula, and anterior cingulate cortex, that then bias processing to favor these stimuli. From their perspective, the amygdala helps prioritize emotional aspects of information processing by coordinating activity in other regions involved in selective attention. Thus, in the multiple waves model, emotion influences general-purpose perceptual and attention systems rather than harnessing independent brain mechanisms to enhance perception of emotional items.

The latter perspective is more compatible with our findings than are separate-system models; if emotional stimuli were processed via a system separate from that processing neutral stimuli, it is not clear how emotional arousal could have both enhancing and impairing effects on neutral stimuli depending on their priority. However, even this modulatory multiple waves approach to emotion-cognition interactions fails to explain the full picture of how emotional arousal influences cognitive processing, as it focuses only on the enhanced perception of arousing stimuli and ignores how arousal affects perceptual selectivity more generally.

\subsection{Canonical amygdala modulation model of emotional memory enhancement}

The act of noticing something creates initial trace representations that require additional resources over the next few minutes, hours, and days to consolidate into a longerlasting memory. Much research indicates that emotional arousal experienced before, during, or after an event can enhance these memory consolidation processes (Hermans et al. 2014). The prevailing view of how emotion affects memory consolidation is that the amygdala enhances processes in the hippocampus and other memory-related brain regions in the medial temporal lobes, such that memory for emotional events is enhanced compared with memory for neutral events (e.g., McGaugh 2004). 
Mather et al.: Norepinephrine ignites local hotspots of neuronal excitation

Table 1. Brain-based emotion-cognition theories.

\begin{tabular}{|c|c|c|}
\hline & Description & Inconsistent/unexplained findings \\
\hline \multicolumn{3}{|c|}{ Models that focus on enhancement of emotionally salient stimuli } \\
\hline $\begin{array}{l}\text { Multiple attention gain control model } \\
\quad \text { (Pourtois et al. 2013) }\end{array}$ & $\begin{array}{l}\text { The amygdala and other modulatory } \\
\text { regions amplify emotionally salient } \\
\text { signals in the sensory pathway in } \\
\text { parallel with bottom-up and top- } \\
\text { down systems. }\end{array}$ & $\begin{array}{l}\text { Emotional arousal can enhance } \\
\text { perception of not only emotional } \\
\text { information, but also non-emotional } \\
\text { information. }\end{array}$ \\
\hline $\begin{array}{l}\text { Multiple waves model (Pessoa \& Adolphs } \\
\text { 2010) }\end{array}$ & $\begin{array}{l}\text { The amygdala and other modulatory } \\
\text { regions coordinate activity in } \\
\text { attention systems to enhance } \\
\text { perception. }\end{array}$ & $\begin{array}{l}\text { Emotional arousal does not always } \\
\text { enhance perception. }\end{array}$ \\
\hline $\begin{array}{l}\text { Amygdala modulation hypothesis } \\
\text { (McGaugh 2004) }\end{array}$ & $\begin{array}{l}\text { The amygdala enhances processing in } \\
\text { other memory-related regions to } \\
\text { enhance memory for emotional } \\
\text { events via noradrenergic } \\
\text { mechanisms. }\end{array}$ & $\begin{array}{l}\text { Norepinephrine-amygdala interactions } \\
\text { enhance memory not only for } \\
\text { emotional stimuli, but also for non- } \\
\text { emotional stimuli. }\end{array}$ \\
\hline \multicolumn{3}{|c|}{ Models that address selective effects of emotion } \\
\hline $\begin{array}{l}\text { Biased attention via norepinephrine } \\
\text { (BANE) model (Markovic et al. 2014) }\end{array}$ & $\begin{array}{l}\text { The anterior affective system detects } \\
\text { emotional saliency and recruits the } \\
\text { locus coeruleus-norepinephrine } \\
\text { system to bias attention and memory } \\
\text { in favor of emotionally salient } \\
\text { stimuli. }\end{array}$ & $\begin{array}{l}\text { Emotional information sometimes } \\
\text { enhances perception and memory } \\
\text { for nearby neutral information. }\end{array}$ \\
\hline Dual competition model (Pessoa 2009) & $\begin{array}{l}\text { Emotional stimuli compete for } \\
\text { resources with other stimuli, leaving } \\
\text { fewer resources available for non- } \\
\text { emotional stimuli. }\end{array}$ & $\begin{array}{l}\text { Emotional information sometimes } \\
\text { enhances perception and memory } \\
\text { for nearby neutral information. }\end{array}$ \\
\hline $\begin{array}{l}\text { Ruthless competition model (Diamond } \\
\text { et al. 2005) }\end{array}$ & $\begin{array}{l}\text { Encoding new emotional information } \\
\text { suppresses recently potentiated } \\
\text { synapses, resulting in enhanced } \\
\text { memory for emotional information at } \\
\text { the cost of preceding events. }\end{array}$ & $\begin{array}{l}\text { Emotional arousal enhances memory } \\
\text { for what occurred earlier if the } \\
\text { preceding event is emotional. }\end{array}$ \\
\hline $\begin{array}{l}\text { Emotional-tagging hypothesis (Richter- } \\
\text { Levin \& Akirav 2003) }\end{array}$ & $\begin{array}{l}\text { Memories for emotional events are } \\
\text { tagged, which allows for subsequent } \\
\text { arousal to selectively enhance } \\
\text { memory for preceding emotional } \\
\text { events. }\end{array}$ & $\begin{array}{l}\text { Emotional arousal can produce } \\
\text { retrograde enhancement even when } \\
\text { preceding information is non- } \\
\text { emotional. }\end{array}$ \\
\hline
\end{tabular}

Consistent with this idea, activity in the amygdala during encoding predicts later memory for emotional items, but not memory for neutral items, as does greater amygdala functional connectivity with medial temporal brain regions (Dolcos et al. 2004; Kilpatrick \& Cahill 2003; Richardson et al. 2004; Ritchey et al. 2008).

Converging rodent and human research indicates that NE facilitates the amygdala-mediated enhancement of emotional information. For example, NE released in the amygdala during arousal is associated with enhanced memory for the emotionally arousing event (McIntyre et al. 2002). Infusion of noradrenergic agonists into the basolateral amygdala after training also enhances memory for emotionally arousing events (Hatfield \& McGaugh 1999; LaLumiere et al. 2003). In humans, administration of the $\beta$-adrenergic antagonist propranolol impairs emotional memories, whereas pharmacological agents that increase NE levels, such as a selective NE reuptake inhibitor, tend to enhance them (Chamberlain \& Robbins 2013), and enhanced amygdala activity during encoding emotional stimuli is reduced by propranolol (Strange \& Dolan 2004). Thus, NE-amygdala interactions enhance memory for emotional events.

Activation of the amygdala by NE can also impair memory for neutral information encountered near something emotional. For example, as already described earlier in the context of the Sakaki et al. (2014a) study, people often have worse memory for neutral-low priority information shown immediately before an emotional compared with a neutral "oddball" stimulus. Patients with amygdalar damage do not exhibit decrements in memory for neutral words preceding emotional oddball words, and in normal individuals, a $\beta$-adrenergic antagonist prevents this retrograde memory impairment (Strange et al. 2003).

Although not usually articulated, the amygdala modulation hypothesis presumably explains these impairment effects for neutral stimuli in terms of a trade-off in which the amygdala focuses resources on emotional stimuli, leaving fewer resources available to process and consolidate 
Mather et al.: Norepinephrine ignites local hotspots of neuronal excitation

the neutral stimuli. However, this trade-off explanation fails to explain how NE-amygdala interactions can also enhance memory for nonarousing information (e.g., Barsegyan et al. 2014; Roozendaal et al. 2008).

\subsection{Biased attention via norepinephrine model}

In the biased attention via norepinephrine (BANE) model, Markovic et al. (2014) propose that affectively salient stimuli activate the LC-NE system to optimize their own processing. Like the ABC model (Mather \& Sutherland 2011), the BANE model builds on biased competition models of attention (Markovic et al. 2014). The BANE model proposes that affect-biased attention "is distinct from both 'classic' executive top-down and bottom-up visual attention and is at least in part circumscribed by a different set of neural mechanisms" (Markovic et al. 2014, p. 230). In the BANE model, emotional salience is detected by an "anterior affective system," including the amygdala and the orbitofrontal cortex, based on the recent history of reward and punishment. In turn, the amygdala's recruitment of the LC-NE system serves as an additional specialized pathway that further biases attention and memory in favor of the affectively relevant information that triggered $\mathrm{NE}$ release. However, like other models of emotion and cognition, the BANE model focuses exclusively on how affectively salient stimuli outcompete less salient stimuli and does not address how arousal induced by these stimuli sometimes enhances and sometimes impairs processing of proximal neutral information.

\subsection{Emotional attention competes with executive attention for limited mental resources}

Another line of work focuses on how emotional stimuli compete for executive resources (Bishop 2007; Choi et al. 2012; Eysenck et al. 2007), with some researchers positing that a ventral affective system competes with a dorsal executive system (Bush et al. 2000; Dolcos et al. 2011). For example, when task-irrelevant emotional stimuli capture attention, they diminish dorsal executive brain region function and therefore disrupt working memory for neutral faces that were just seen (Dolcos \& McCarthy 2006; Dolcos et al. 2008). However, metaanalyses indicate that emotional responses are associated with both the ventral and dorsal prefrontal cortical regions (Phan et al. 2002; Shackman et al. 2011), and so the notion that emotional distractors lead the ventral prefrontal cortical region to inhibit the dorsolateral prefrontal cortical region (Dolcos et al. 2008) is unlikely to be universal across different contexts.

Instead of a ventral/dorsal antagonism model, the dual competition model posits that emotional stimuli compete for resources at both perceptual and executive levels of processing (Pessoa 2009; 2013). For example, when participants heard tones predicting shock, regions within the frontoparietal network were activated (Lim et al. 2009). Recruitment of these regions during intense emotional arousal should make them less available for concurrent neutral task-related processing and lead to behavioral impairments. At the perceptual level of the dual competition model, both cortical and subcortical structures help amplify visual cortex responses to emotional stimuli, again leading to the impaired perception of other concurrent stimuli.

As in the $\mathrm{ABC}$ framework, competition is a core feature of these models. These models, however, consider only one type of competition: that between arousing and neutral stimuli/tasks. Critically, our empirical results indicate that arousal also influences competition between two neutral stimuli, such that processing of high-priority stimuli is enhanced, whereas processing of lower-priority stimuli is impaired. It is not clear how, in competition models that focus on competition between arousing and neutral stimuli, arousal would interact differently with low- and high-priority neutral information. For example, such models cannot account for the differential effects of arousing sounds on subsequent perceptually salient versus nonsalient letters (Fig. 1).

\subsection{Competition between items for memory consolidation}

In a different type of competition account, Diamond et al. (2005) propose that there is "ruthless competition" between novel and existing memory representations, such that encoding a new emotional experience suppresses recently potentiated synapses, creating memory for emotional events at the cost of memory for information learned just before the emotional event (Diamond et al. 2005).

This ruthless competition hypothesis argues that the acquisition of new information via the hippocampus depotentiates the most recently activated synapses and that this suppression of recently formed memories is greater when the new information induces emotion or stress. Thus, inducing arousal should impair memory for a preceding sequence of items, regardless of whether those preceding items were themselves emotional or not. That is not the case, however. Inducing arousal via emotional or coldpressor stress immediately after participants study a mixed list of emotional and neutral pictures selectively enhances memory for preceding emotional, but not neutral, pictures (Cahill et al. 2003; Liu et al. 2008).

\subsection{An arousing stimulus sometimes impairs and sometimes enhances memory of what just happened}

How can inducing arousal enhance memory for preceding emotional items but not neutral items? Investigators proposed that emotional arousal "tags" synapses associated with representations of emotional items, making these synapses the selective target of protein synthesis-dependent long-term potentiation (Bergado et al. 2011; RichterLevin \& Akirav 2003; Segal \& Cahill 2009; Tully \& Bolshakov 2010). The emotional tagging hypothesis predicts that emotionally salient stimuli are remembered better than neutral stimuli because emotional tags allow those particular synapses to capture the plasticity-related proteins released with subsequent inductions of arousal.

A problem for the emotional tagging model is that inducing emotional arousal sometimes enhances memory for preceding neutral stimuli (Anderson et al. 2006; Dunsmoor et al. 2015; Knight \& Mather 2009; Nielson \& Powless 2007; Sakaki et al. 2014a). Neither the emotional tagging hypothesis nor any of the other hypotheses outlined 
earlier can account for this retrograde enhancement of something neutral. In contrast to the emotional tagging hypothesis, behavioral studies demonstrate that whether something arousing will yield retrograde enhancement or impairment depends on the priority of the preceding information (sect. 2.5) (Ponzio \& Mather 2014; Sakaki et al. 2014a).

\subsection{Summary}

Although there are many models describing how emotion enhances perception, attention, and memory in the brain, these theories fail to account for both the enhancing and impairing effects of emotional arousal (see Table 1 for a summary). In the following sections, we make the case for GANE, a model of how NE released under arousal can impact high- and low-priority representations differently despite its diffuse release across the brain.

\section{Locus coeruleus, NE, and arousal}

Like the GANE model, other theories also argue that the LC-NE system is important for emotion-cognition interactions (Markovic et al. 2014; McGaugh 2000; 2004; McIntyre et al. 2012). However, they have focused mostly on how NE interacts with the amygdala to enhance processing and consolidation of emotional stimuli at the expense of processing neutral stimuli (e.g., Strange \& Dolan 2004; Strange et al. 2003). In contrast, we argue that the LC$\mathrm{NE}$ system promotes selectivity for any prioritized stimuli, irrespective of whether they are emotional or nonemotional.

In this section, we review the functional anatomy of the LC-NE system. A small nucleus in the brainstem known as the locus coeruleus (LC) releases NE when people are aroused - whether by a reward or punishment, a loud noise, or a disturbing image. LC axons are distributed throughout most of the brain (Gaspar et al. 1989; JavoyAgid et al. 1989; Levitt et al. 1984; Swanson \& Hartman 1975), enabling NE to modify neural processing both locally and more globally in large-scale functional brain networks. How does the LC influence information processing in most cortical and subcortical regions? One might think that a hormone released under conditions of arousal would amp up brain activity. But instead, NE quiets most neuronal activity. In turn, this quiet backdrop makes those select few representations that NE amplifies stand out even more.

\subsection{Functional neuroanatomy of the LC-NE system}

The LC is the primary source of cortical NE and helps determine arousal levels (Berridge \& Waterhouse 2003; Berridge et al. 2012; Samuels \& Szabadi 2008a; 2008b). Tonic, or background, levels of LC activity help regulate levels of wakefulness (Carter et al. 2010). Phasic, or transient, bursts of LC activity occur in response to novel, stressful, or salient stimuli (Aston-Jones \& Bloom 1981; Foote et al.1980; Grant et al. 1988; Sara \& Bouret 2012; Sara \& Segal 1991; Vankov et al. 1995) or to top-down signals associated with decision outcomes or goal relevance (Aston-Jones \& Cohen 2005; Aston-Jones et al. 1999). Emotionally salient stimuli also induce LC phasic activity irrespective of whether stimuli are positive (Bouret \& Richmond 2015; Grant et al. 1988) or aversive (Chen \& Sara 2007; Grant et al. 1988).

With highly divergent branching axons, the LC projects to every major region of cortex, despite its relatively small number of neurons (13,000 per hemisphere in humans) (Foote \& Morrison 1987). Subcortical regions that underlie memory, attention, and emotional processing, including the hippocampus, frontoparietal cortex, and amygdala, are also innervated by the LC (Berridge \& Waterhouse 2003). LC axon varicosities release NE into extracellular space, allowing it to activate a broad swath of receptors within a diffusion zone (Beaudet \& Descarries 1978; Descarries et al. 1977; O’Donnell et al. 2012).

In target brain sites, $\mathrm{NE}$ binds to multiple receptor subtypes (i.e., $\alpha_{1}, \alpha_{2}$, and $\beta$ receptors) that are located both pre- and postsynaptically on neurons and astrocytes (Berridge \& Waterhouse 2003; O'Donnell et al. 2012; Terakado 2014; Tully \& Bolshakov 2010). Whereas $\alpha_{2}$ adrenoreceptors limit global and local NE release by acting as autoreceptors and decrease cell excitability, $\beta$ adrenoreceptor activation generally increases cell excitability, network activity, and synaptic plasticity (Berridge \& Waterhouse 2003; Marzo et al. 2009; Nomura et al. 2014; Starke 2001). $\alpha_{1}$-Adrenoreceptors recruit phospholipase activation and typically increase cell excitability via the inhibition of potassium channels (Wang \& McCormick 1993). Thus, the relative density and localization of adrenoreceptor subtypes help determine how arousal-induced NE release will affect neural processing in different brain regions.

\subsection{NE decreases neuronal noise in sensory regions during arousal}

In the 1970s, researchers proposed that LC-NE activity enhances signal-to-noise ratios in target neurons in sensory regions (Foote et al. 1975; Freedman et al. 1977; Segal \& Bloom 1976; Waterhouse \& Woodward 1980). For example, recording from individual neurons in awake squirrel monkeys revealed that NE application reduced spontaneous activity more than it reduced activity evoked by species-specific vocalizations (Foote et al. 1975). Noradrenergic regulation of signal-to-noise ratios is characterized by two simultaneous effects: (1) most neurons in a population decrease spontaneous firing, and (2) the few neurons that typically respond strongly to the specific current sensory stimuli either show no decrease or an increase in firing, unlike the majority of neurons for which the stimuli typically evoke weak responses (Foote et al. 1975; Freedman et al. 1977; Hasselmo et al. 1997; Kuo \& Trussell 2011; Livingstone \& Hubel 1981; O’Donnell et al. 2012; Oades 1985; Waterhouse \& Woodward 1980).

Intracellular recording data in awake animals support and extend these early observations. Both inhibitory and excitatory neurons are depolarized in aroused cortex when mice run (Polack et al. 2013). Yet, consistent with earlier reports of a quieter cortex under arousal, inhibitory neurons are more depolarized than excitatory neurons (Polack et al. 2013). Moreover, surround inhibition dominates sensory responses during wakefulness compared with anesthesia, increasing the speed and selectivity of responses to stimuli in the center of the receptive field (Haider et al. 2013). NE mediates the increase in widespread 
Mather et al.: Norepinephrine ignites local hotspots of neuronal excitation

depolarization and the increase in inhibitory activity in visual cortex that together increase the signal-to-noise ratio (Polack et al. 2013). The effect of NE has also been characterized as increasing the gain on the activation function of neural networks (Fig. 5) (Aston-Jones \& Cohen 2005).

Arousal is also characterized by cortical desynchronization, both globally when comparing wakefulness with anesthesia (Constantinople \& Bruno 2011) or locomotion with being stationary (Polack et al. 2013) and locally among neurons corresponding to attended representations (Fries et al. 2001). Such decreases in cortical slow wave synchrony under arousal are likely mediated by LC activity (Berridge \& Foote 1991; Berridge et al. 1993). Synchronous slow wave neural activity may gate sensory inputs, whereas desynchronized activity permits communication of cortical representations of stimuli across the brain (Luczak et al. 2013). Cortical cell depolarization, desynchronization, and increased responsiveness to external input also occur with pupil dilation (Reimer et al. 2014; Vinck et al. 2014), and pupil dilation tracks LC activity (Murphy et al. 2014).

\subsection{Summary}

Years of research indicate that NE suppresses weak or random neuronal activity, but not strong activity. This is consistent with the increased selectivity seen under arousal (sect. 2). In the next section, we outline a model of how NE has such different outcomes depending on activity level.

\section{Glutamate amplifies noradrenergic effects: The core noradrenergic selectivity mechanism under arousal}

Now we turn to our GANE model, a novel brain-based account of how arousal amplifies priority effects in perception and memory. We propose that local glutamate-NE interactions increase gain under arousal. Glutamate is the most prevalent excitatory neurotransmitter in the brain (Meldrum 2000). Glutamate receptors such as $\alpha$-amino-3hydroxy-5-methyl-4-isoxazolepropionic acid (AMPA) and $N$-methyl-D-aspartate (NMDA) receptors mediate rapid

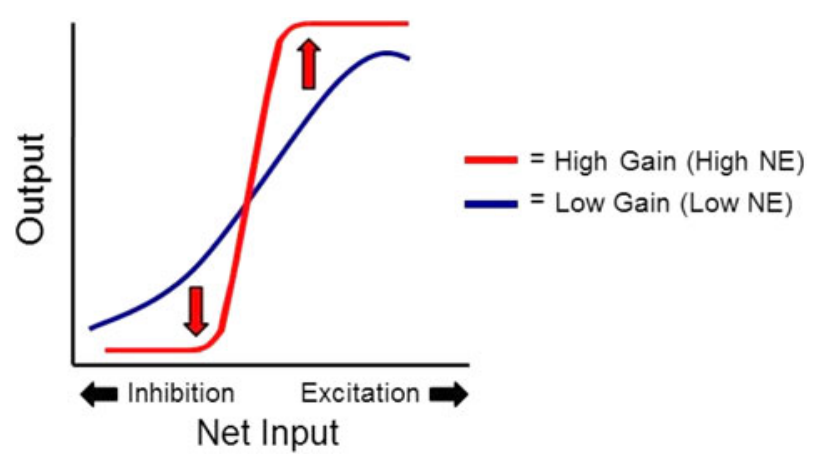

Figure 5. Norepinephrine gain modulation makes the nonlinear input-output function more extreme, increasing the activity of units receiving excitatory input and decreasing the activity of units receiving inhibitory input. Adapted from AstonJones and Cohen (2005). excitatory synaptic transmission, neural network connectivity, and long-term memory (Bliss \& Collingridge 1993; Lynch 2004; Traynelis et al. 2010).

In addition to point-to-point transmission across a synapse, some glutamate escapes the synaptic cleft, resulting in "glutamate spillover" (Okubo et al. 2010). In this section, we outline evidence that glutamate spillover attracts and amplifies local NE release via positive feedback loops. These self-regulating NE hotspots generate even greater excitatory activity in the vicinity of synapses transmitting high-priority representations, in contrast with NE's suppressive effects in the more widespread nonhotspot regions.

\subsection{The NE hotspot: How local NE-glutamate positive feedback loops amplify processing of high-priority information}

5.1.1 High glutamate activity stimulates adjacent NE varicosities to release more NE. The first demonstrations of glutamate-evoked effects on NE found that glutamate increased NE release via NMDA and non-NMDA glutamate receptors on LC axons (Fink et al. 1989; Göthert \& Fink 1991; Lalies et al. 1988; Nelson et al. 1980; Pittaluga \& Raiteri 1990; 1992; Vezzani et al. 1987; Wang et al. 1992; see also Jones et al. 1987). In these studies, glutamateevoked NE release occurred for NE varicosities in all cortical structures investigated in vitro: olfactory bulb, hippocampus, and throughout neocortex. In vivo experiments replicated the effect with targeted glutamate in rodent prefrontal cortex (Lehmann et al. 1992). Other neurotransmitters associated with arousal, such as histamine (Burban et al. 2010) and orexin (Tose et al. 2009), enhance glutamate-evoked NE release. Central to our hypothesis, glutamate-evoked NE release occurs in human neocortex (Fink et al. 1992; Luccini et al. 2007; Pittaluga et al. 1999).

How do these glutamate-NE interactions occur? LC axon varicosities rarely make direct synaptic contacts (e.g., only $\sim 5 \%$ in rat cortex) (Vizi et al. 2010), but the distribution of these varicosities suggests they should often be found near glutamate terminals at excitatory synapses in neocortex (Benavides-Piccione et al. 2005; Gaspar et al. 1989). Another critical point is that LC neurons produce the NMDA receptor subunits needed for glutamate to modulate the release of NE from LC axon varicosities (Chandler et al. 2014; Grilli et al. 2009; Petralia et al. 1994; Zhu et al. 2003).

New technologies enable the visualization of glutamate spillover in cerebellum, neocortex, and hippocampus (Okubo et al. 2010; Okubo \& Iino 2011). Multiple action potentials in a row yield sufficient spillover glutamate to activate nonsynaptic NMDA and group I metabotropic glutamate receptors (mGluRs) (which are co-expressed on NE varicosities and enhance glutamate-evoked NE release in rodent and human cortices [Luccini et al. 2007]), but probably yield insufficient glutamate to recruit lower-affinity AMPA receptors (Okubo et al. 2010). Extracellular concentrations of the spillover rapidly decrease as distance from the synaptic cleft increases (Vizi et al. 2010), and the upper limit of glutamate spillover effects is estimated to be no greater than a few micrometers (Okubo \& Iino 2011).

That spillover glutamate is sufficient to activate NMDA, but not AMPA receptors is another key factor. Unlike 
AMPA receptors, NMDA receptors require synchronized glutamate stimulation and neuron depolarization to activate (Lüscher \& Malenka 2012). Thus, local glutamate spillover must co-occur with phasic depolarizing bursts of activity in LC neurons to recruit additional local NE release. Furthermore, a unique feature of NMDA receptors is that they require a co-agonist, which could be either glycine or Dserine (Wolosker 2007). Glutamate stimulates astrocytes to release these co-agonists (Harsing \& Matyus 2013; Van Horn et al. 2013), and both glutamate and NE stimulate astrocytes to release glutamate (Parpura \& Haydon 2000). These additional glutamate interactions should further enhance NMDA receptor-mediated NE release (Fig. 6) (Paukert et al. 2014). Together, these local glutamate$\mathrm{NE}$ interactions support the emergence and sustainment of hotspots in the vicinity of the most activated synapses when arousal is induced.

Consistent with the existence of glutamate-NE interactions, local NE release in the region of an activated novel representation depends on the coincident timing of the novel event and an arousing event (Rangel \& Leon 1995). For example, when footshock was administered to a rat while it explored a novel environment, NE levels rose substantially higher and remained elevated longer than when

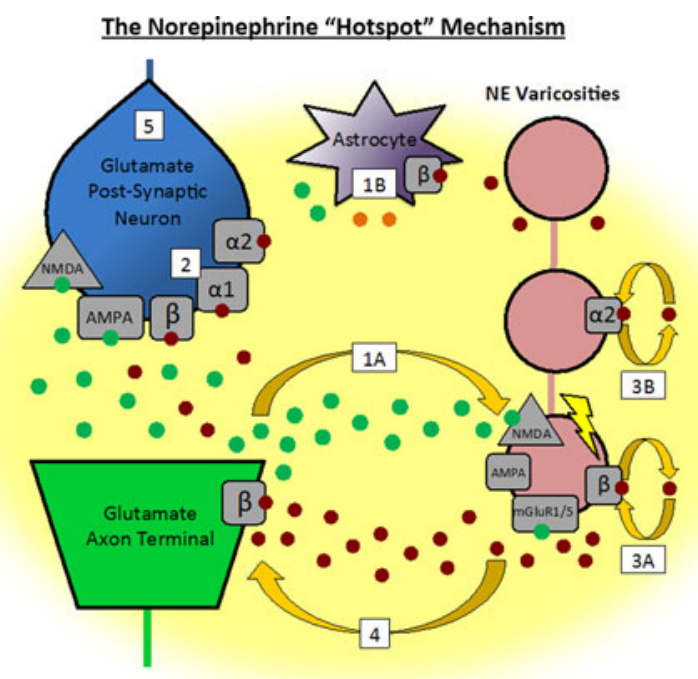

Figure 6. Norepinephrine (NE) "hotspot" mechanism. (1A) Spillover glutamate (green dots) from highly active neurons interacts with nearby depolarized $\mathrm{NE}$ varicosities in a positive feedback loop involving $N$-methyl-D-aspartate (NMDA) and other glutamate receptors that leads to greater local NE release (maroon dots). The glutamatergic NMDA receptors require concomitant depolarization of noradrenergic axons (lightning symbol). Thus, hotspots amplify prioritized inputs most effectively under phasic arousal. (1B) Glutamate also recruits nearby astrocytes to release serine, glycine (orange dots), and additional glutamate. (2) Greater NE release creates concentration levels sufficient to activate low-affinity $\beta$ adrenoreceptors, which enhances neuron excitability. (3) Via activation of $\beta$ - and $\alpha_{2 \mathrm{~A}}$-auto-receptors, $\mathrm{NE}$ can stimulate and inhibit additional NE release, respectively. (4) Within hotspots, $\mathrm{NE}$ engages $\beta$-adrenoreceptors on pre-synaptic glutamate terminals to increase glutamate release. (5) Finally, NE binding to postsynaptic $\beta$-adrenoreceptors also inhibits the slow afterhyperpolarization, enabling the neuron to fire even longer. AMPA $=\alpha$-amino-3-hydroxy-5-methyl-4-isoxazolepropionic acid; $\mathrm{mGluR}=$ metabotropic glutamate receptor. footshock was administered to the rat in its holding cage (Fig. 7) (McIntyre et al. 2002). The amygdala presumably activated in response to the novelty of the new environment (Weierich et al. 2010), and glutamate associated with that representational network amplified the NE release initiated by the shock.

Hotspot effects have also been observed in the bed nucleus of the stria terminalis immediately after training rats on an inhibitory avoidance task (Liu et al. 2009). When infused separately at low doses, glutamate and NE each had no effect. But when infused together at the same low doses, they produced marked memory enhancements. Infusion of a higher dose of glutamate led to memory enhancements that were blocked by propranolol, indicating that the glutamate effect required $\beta$-adrenergic activity, which, as we describe next, is another key feature of our hotspot model.

\subsection{2. $\alpha$ - and $\beta$-adrenoreceptors exert different effects on} neuronal excitability and require different NE concentrations to be activated. To be engaged, $\beta$-adrenoreceptors require relatively high $\mathrm{NE}$ concentrations, $\alpha_{1}$-adrenoreceptors more moderate levels, and $\alpha_{2}$-adrenoreceptors the lowest NE concentrations (Ramos \& Arnsten 2007). Thus, under arousal, $\alpha_{2}$-adrenoreceptor effects should be widespread, whereas $\beta$-adrenoreceptors should be activated only at hotspot regions because the local glutamateevoked NE release there results in higher NE levels. Next, we describe the importance of this distinction for adrenergic autoreceptors.

5.1.3 Adrenergic autoreceptors inhibit or amplify their own NE release. Autoreceptors at NE varicosities serve as neural gain amplifiers by taking opposing action at low and high local levels of NE. The predominant presynaptic noradrenergic autoreceptor in humans is the $\alpha_{2 \mathrm{~A}}$-adrenoreceptor (Starke 2001), which inhibits NE release when it detects low or moderate levels of NE (Delaney et al. 2007; Gilsbach \& Hein 2008; Langer 2008; Starke 2001). In contrast, presynaptic $\beta$-adrenoreceptors amplify $\mathrm{NE}$ release when activated by high levels of NE (Chang et al. 1986; Misu \& Kubo 1986; Murugaiah \& O’Donnell 1995a; 1995b; Ueda et al. 1985). In addition, $\alpha_{2 \mathrm{~A}}$-adrenoreceptors may lose affinity for NE when neurons are depolarized (Rinne et al. 2013), which would remove their inhibitory influence as a region becomes highly active. However, this loss of affinity recovers at saturating levels of NE (Rinne et al. 2013), which should help prevent the runaway excitation that could otherwise emerge because of the NE-glutamate feedback loop. Together with glutamate-evoked NE release (see sect. 5.1.1), the opposing effects of these different autoreceptors at low and high levels of NE provide an elegant way for the LC to modulate signal gain depending on the degree of local excitation.

5.1.4 Elevated local NE at hotspots engages $\beta$-adrenoreceptors on the glutamate terminals transmitting the prioritized representation. This stimulates an even greater release of glutamate, thereby amplifying the high-priority excitatory signal (Ferrero et al. 2013; Gereau \& Conn 1994; Herrero \& Sánchez-Prieto 1996; Ji et al. 2008; Kobayashi et al. 2009; Mobley \& Greengard 1985). That $\beta$-adrenoreceptors require relatively high $\mathrm{NE}$ concentrations to 
Mather et al.: Norepinephrine ignites local hotspots of neuronal excitation

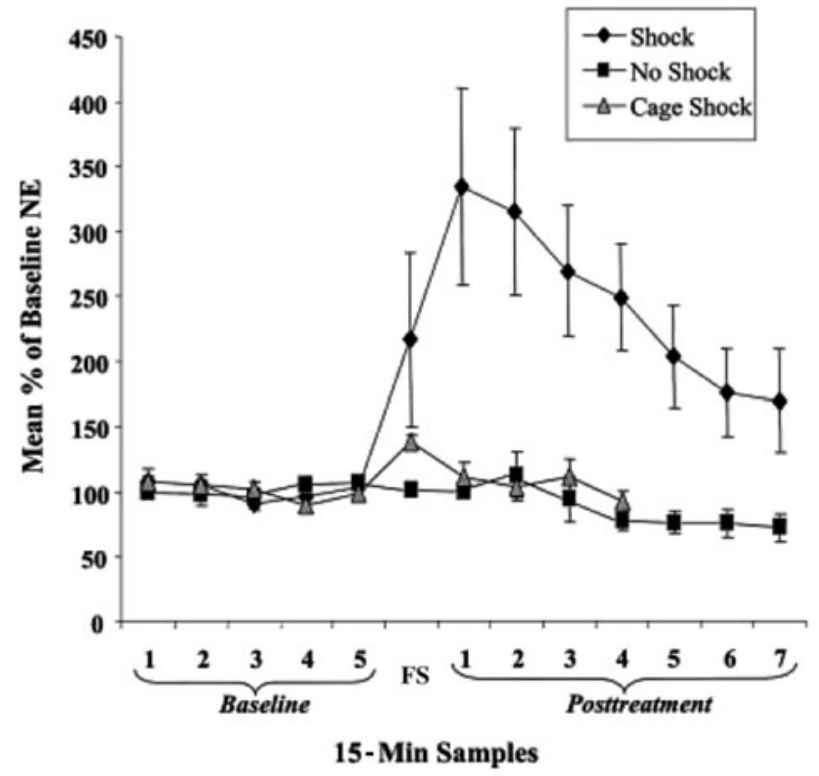

Figure 7. A rat receiving a foot shock (FS) in its home cage exhibits a brief increase in norepinephrine (NE) levels (gray triangles). A novel training environment does not increase $\mathrm{NE}$ on its own (black squares), but NE levels increase dramatically when shock is combined with that novel training environment (black diamonds). Figure reprinted with permission from McIntyre et al. (2002).

be engaged further biases this form of cortical autoregulation towards the most active synapses. Through these feedback processes, high-priority representations are "self-selected" to produce a stronger glutamate message and excite their connections more effectively under arousal. This stronger glutamate message should also promote selective memory of such stimuli (see sect. 6.1). In contrast, activation of lower threshold $\alpha_{2}$-adrenoreceptors inhibits glutamate release (Bickler \& Hansen 1996; Egli et al. 2005), providing a mechanism for inhibiting lower-priority neural activity under arousal.

5.1.5 Higher NE levels at hotspots help prolong the period of neuronal excitation by temporarily inhibiting processes that normalize neuron activity. Under normal conditions, the slow after-hyperpolarization current habituates a postsynaptic neuron's responses following prolonged depolarization (Alger \& Nicoll 1980). However, even here, NE seems to benefit prioritized inputs by prolonging neuronal excitation via $\beta$-adrenoreceptors inhibiting the slow afterhyperpolarization (Madison \& Nicoll 1982; Nicoll 1988).

In summary, different receptor subtypes enable NE to ignite hotspots in regions with high glutamate levels while inhibiting activity elsewhere. As we outline later, this diversity in NE receptor subtypes also plays an important role in shaping synaptic plasticity to favor prioritized representations under phasic arousal.

\subsection{NE hotspots modulate interneurons and GABAergic transmission to increase lateral inhibition of competing representations}

Increases in glutamate and NE at hotspots should also enhance inhibitory activity that mediates competition among neurons. $\gamma$-Aminobutyric acid (GABA) is the most widespread inhibitory transmitter from neurons that suppress the responses of other neurons or neuronal circuits (Petroff 2002). Strong glutamate activity in cortical circuits stimulates local GABAergic activity, which increases the inhibitory effects of highly active regions on neighboring, competing neural circuits (Xue et al. 2014). Increases in $\mathrm{NE}$ also activate inhibition directly, with intermediate concentrations engaging maximal suppression (Nai et al. 2009).

Subtypes of interneurons respond differently to NE in ways that should further increase neural gain. Although LC-NE activity activates interneurons that mediate lateral inhibition (Salgado et al. 2012a), it can also suppress interneurons with feedforward connections (Brown et al. 2005), such that a strong signal will inhibit competing representations while enhancing activity in other neurons within its processing pathway.

\subsection{NE directs metabolic resources to where they are most needed}

To optimize processing of salient events, NE also helps coordinate the delivery of the brain's energy supplies, allowing it to mobilize resources quickly when needed (e.g., Toussay et al. 2013). The brain's most essential energy supplies, oxygen and glucose, are delivered via the bloodstream. One key way that NE coordinates energy delivery is by increasing the spatial and temporal synchronization of blood delivery to oxygen demand within the brain. For example, in mice, as NE levels increase, overall blood vessel diameter in the brain decreases, but the spatial and temporal selectivity of blood distribution to active task-relevant regions increases (Bekar et al. 2012).

In addition to distributing blood flow, NE also interacts with astrocytes locally to mobilize energy resources throughout the cortex. When a particular area of the brain needs more energy, it can obtain fuel not only from glucose, but also from glycogen in astrocytes (Pellerin \& Magistretti 2012). NE speeds up the process of obtaining energy from glycogen (Magistretti et al. 1981; Sorg \& Magistretti 1991; Walls et al. 2009). While $\alpha_{1^{-}}$and $\alpha_{2^{-}}$ adrenoreceptors mediate glutamate uptake and glycogen production in astrocytes, $\beta$-adrenoreceptors stimulate the breakdown of glycogen to provide rapid energy support in highly active local regions (O’Donnell et al. 2012), further amplifying NE hotspot activity.

\subsection{Summary}

At the local neuronal level, NE suppresses most activity, but amplifies the strongest activity as a result of the differential effects of NE on different adrenoreceptor subtypes. The amplification of strong activity occurs via "NE hotspots," where positive feedback loops between local NE and glutamate release increase the strength of activated representations. To sustain higher levels of activity, hotspots also recruit limited metabolic resources. At the circuit level, the increased glutamate and NE produced at hotspots recruit nearby astrocytes that supply additional energy to active neurons. On a broader scale, NE facilitates the redistribution of blood flow towards hotspots and away from areas of lower activity. Thus, by influencing multiple levels of brain function, NE selectively amplifies self- 
regulating processes that bias processing in favor of prioritized information.

\section{Roles of the LC-NE system in memory}

So far we have focused on how arousal increases the gain on prioritization processes in perception, attention, and initial memory encoding. Now we turn to memory consolidation processes. Experiencing an emotionally intense event influences the vividness and longevity of recent memory traces, enhancing or impairing them based on their priority (e.g., Fig. 4) (Knight \& Mather 2009; Sakaki et al. 2014a). Much research has indicated that $\mathrm{NE}$ is involved in memory consolidation effects (for a review, see McGaugh [2013]), but there has been little focus on the interplay between NE's enhancing and impairing effects during memory consolidation.

The durability of memories depends on adjustments in the strength of communication across synapses via processes known as long-term potentiation (LTP) and longterm depression (LTD). Whether neural activity triggers LTP or LTD depends on the relative timing of spikes in pre- and postsynaptic neurons (Nabavi et al. 2014), and whether LTP and LTD are maintained depends on protein synthesis processes (Abraham \& Williams 2008). We propose that two main NE mechanisms modulate LTP and LTD, leading to "winner-take-more" and "losertake-less" outcomes in long-term memory: (1) hotspot modulation of the probability of LTP (higher NE levels engaging LTP) and LTD (relatively lower NE levels promoting LTD), and (2) NE-enhanced protein synthesis supporting long-term maintenance of LTP and LTD.

\subsection{NE gates spike-timing-dependent LTD and LTP}

Long-term potentiation and long-term depression are often studied in brain slices in a petri dish using high-frequency electric stimulation to induce LTP and repeated slow stimulation to induce LTD. But in the brain's natural context involving constant barrages of pre-synaptic activity generating postsynaptic spikes, the relative timing of pre- and postsynaptic activity helps determine whether LTP or LTD occurs. Furthermore, to avoid constant up-and-down adjustment of synapses based on random firing patterns, neuromodulators such as NE and dopamine signal when the relationship between pre-synaptic and postsynaptic activity is likely to be meaningful (Pawlak et al. 2010). In vivo studies indicate that spike-timing-dependent LTP or LTD requires these neuromodulators (Huang et al. 2014; Johansen et al. 2014). In particular, by binding to G-coupled receptors, NE modulates kinases and phosphatases that determine whether LTP or LTD induction occurs (Treviño et al. 2012b; Tully \& Bolshakov 2010).

Different adrenoreceptor subtypes appear to mediate NE's regulation of spike-timing-dependent LTP and LTD. Spike-timing-dependent LTP is initiated primarily by $\beta$-adrenoreceptor activation, whereas $\alpha_{1}$-adrenoreceptors promote spike-timing-dependent LTD (Salgado et al. $2012 b)$. Critically, Salgado and colleagues reported that the LTP promoting activation of $\beta$-adrenoreceptors requires concentrations of $\mathrm{NE} \sim 25$-fold higher $(8.75 \mu \mathrm{M})$ than the NE concentration that promotes $\alpha_{1}$-adrenoreceptor-mediated spike-timing-dependent LTD $(0.3 \mu \mathrm{M})$ in vitro. This agrees with an in vivo estimate of a 30-fold increase in NE associated with LTP in dentate gyrus (Harley et al. 1996). The increase in NE required to support spike-timing-dependent LTP is substantially higher than the increases in NE levels seen when experimenters stimulate LC and measure NE in cortex or hippocampus using microdialysis (e.g., approximately twice baseline [Florin-Lechner et al. 1996], $\sim 0.5 \mu \mathrm{M}$ [Palamarchouk et al. 2000]). Thus, there is a discrepancy between the NE levels needed for spike-timing-dependent LTP to occur and the levels measured in laboratory studies. Our GANE model accounts for this difference, as it posits that LC activation interacts with prioritized representations to elicit much higher NE release in a select few local hotspots than elsewhere, such that the average cortical sampling location would not detect the NE levels needed to support LTP.

The NE hotspot model supports a range of simultaneous NE modulatory actions. At high-priority hotspots, NE levels should be sufficiently high to engage $\beta$-adrenoreceptors and initiate spike-timing-dependent LTP (Salgado et al. 2012b; Treviño et al. 2012b). Conversely, areas with lower glutamate activity, where NE levels are by comparison modestly increased, would undergo LTD as a result of the engagement of relatively higher affinity $\alpha_{1}$-adrenergic receptors (Huang et al. 2014; Salgado et al. 2012b; Treviño et al. 2012a). Variations in NE levels in the alert brain thereby support bidirectional plasticity (Salgado et al. 2012b; Treviño et al. 2012b).

\subsection{NE increases protein synthesis processes that promote memory consolidation: Critical role of $\boldsymbol{\beta}$-adrenoreceptors}

Arousal levels in the minutes and hours before or after an event also influence later memory for it. Here we review evidence that these wider time window effects of arousal depend on NE's enhancement of protein synthesis processes that determine the long-term durability of salient memories. Critically, such regulation of memory processes by NE appears to be mediated by $\beta$-adrenoreceptors, which we propose are selectively activated in high-priority representational networks.

The role of NE in gating the synthesis of plasticityrelated proteins has been recognized for more than a decade (Cirelli et al. 1996; Cirelli \& Tononi 2000). For example, plasticity-related proteins promoted by an LC-NE novelty signal can enhance long-term memory consolidation of another salient, but otherwise poorly consolidated event (i.e., learning that stepping off of a platform leads to a weak shock) that occurs 1 hour later or even 1 hour prior to the novelty experience (Moncada \& Viola 2007; Moncada et al. 2011).

Blocking $\beta$-adrenoreceptors or protein synthesis prior to novelty exposure prevents novelty facilitation of LTP (Straube et al. 2003). What is particularly striking is that $\beta$-adrenoreceptor activation at time 1 primes synapses to induce LTP at time 2 an hour later, even when $\beta$-adrenoreceptors are blocked by propranolol during time 2 (Tenorio et al. 2010). However, if protein synthesis processes are blocked during time 2 , the time 1 priming event does not lead to enhancement. The plasticity marker, Arc protein, is recruited by $\beta$-adrenoreceptor activation in the presence of NMDA receptor activation 
Mather et al.: Norepinephrine ignites local hotspots of neuronal excitation

(Bloomer et al. 2008). Hotspots are characterized by high levels of glutamate release and $\beta$-adrenoreceptor activation; thus, emotional arousal should elevate Arc selectively in NE hotspots.

$\beta$-Adrenergic activation after learning or weak LTP induction can also convert short-term LTP to more lasting protein synthesis-dependent late LTP (Gelinas \& Nguyen 2005; Gelinas et al. 2008). Likewise, stimulating the basolateral amygdala either before or after tetanization of the hippocampus converts early LTP to late LTP via a $\beta$-adrenoreceptor mechanism (Frey et al. 2001). Activation of $\beta$-adrenoreceptors also shields late LTP from subsequent depotentiation (Gelinas \& Nguyen 2005; Katsuki et al. 1997).

Creation of long-lasting memories depends on the protein synthesis cyclic adenosine monophosphate $(\mathrm{cAMP}) /$ protein kinase A (PKA)/cAMP response elementbinding protein (CREB) pro-signaling cascade (Kandel 2012; O'Dell et al. 2010). Neuronal ensembles in which the cAMP/PKA/CREB cascade has been activated, as happens with the engagement of $\beta$-adrenoreceptors, have been found to be selectively allocated to the engram representing a memory (Han et al. 2007). Furthermore, increasing excitability via different methods mimics the effects of CREB overexpression, suggesting that neurons are recruited to an engram based on their neural excitability (Frankland \& Josselyn 2015; Zhou et al. 2009). Thus, by modulating CREB and other aspects of neural excitability, NE hotspots should help determine which neurons are allocated to an engram and stabilized in long-term memory.

\subsection{Summary}

Local NE concentration is the key to understanding how NE mediates arousal's dichotomous effects on memory. Previous research has indicated that different NE levels regulate different forms of spike-timing-dependent plasticity by engaging distinct adrenoreceptors. Whereas $\mathrm{NE}$ binding to moderate-affinity $\alpha_{1}$-adrenergic receptors leads to LTD and memory suppression, NE binding to lower-affinity $\beta$-adrenoreceptors leads to LTP and memory enhancement. We propose that local discrepancies in NE levels arise from self-regulating NE-glutamate interactions. Where NE concentrations become high enough to engage low-affinity $\beta$-adrenoreceptors, a cascade of intracellular events triggers protein synthesis processes that enable long-term memory consolidation of the high-priority trace. In contrast, more modest increases in NE levels at less active regions lead to LTD, ensuring less important events are forgotten. Before or after encoding, the confluence of protein synthesis and $\beta$-adrenoreceptor activation selectively strengthens memory consolidation when these mechanisms are recruited close in time.

\section{Beyond local GANE: Broader noradrenergic circuitry involved in increased selectivity under arousal}

Beyond local effects, NE increases biased competition processes by altering how different brain structures interact. With its widely distributed afferents, the LC-NE system influences neural processing in many brain regions when an arousing event occurs. NE release can translate local hotspot effects to more global winnertake-more effects by modulating neuronal oscillations. Furthermore, cortical and subcortical priority signals modulate glutamate release in sensory regions and the hippocampus as mental representations are formed and sustained. As previously reviewed (see sect. 5.1), glutamate is essential for NE release to selectively amplify the processing of significant information. Thus, by stimulating local glutamate release and recruiting LC firing, key brain structures can optimize synaptic conditions for arousal to ignite hotspots.

\subsection{Activation of inhibitory networks by NE primes neuronal synchronization among high-priority neural ensembles}

So far, we have reviewed evidence that NE hotspots amplify the effects of priority, enhancing salient features while suppressing noisy background activity. In this section, we discuss the possibility that neuronal oscillations communicate activity in local hotspots more globally (Singer 1993).

The first candidate is gamma synchrony $(30-80 \mathrm{~Hz})$. Conceptual frameworks of neural oscillations posit that gamma synchrony supports gain modulation in local networks (Fries 2009), such that a target area can oscillate in phase with only one of two competing inputs. As a result, the synaptic input that more successfully synchronizes its activity with the target region is amplified, whereas the less synchronized input is suppressed. Gamma synchrony is likely a key component of selective attention (Baluch \& Itti 2011; Fries 2009; Fries et al. 2001).

Gamma oscillations are generated by a feedback loop between excitatory pyramidal cells and fast-spiking parvalbumin-positive inhibitory interneurons (Buzsáki \& Wang 2012; Cardin et al. 2009; Carlen et al. 2012; Sohal et al. 2009). Noradrenergic release activates these interneurons (Cox et al. 2008; Huang et al. 2013; Toussay et al. 2013) and increases gamma synchrony in these target regions (Gire \& Schoppa 2008; Haggerty et al. 2013; Marzo et al. 2014). Emotional arousal also modulates gamma oscillations in regions that process motivational significance, such as the amygdala, sensory cortex, and prefrontal cortex (Headley \& Weinberger 2013). These results suggest that arousal-induced NE release selectively biases gamma oscillations in favor of the most activated representations in local neuronal ensembles.

Consistent with the hotspot model, increases in local gamma power during cognitive processing in humans are associated with increases in glutamate levels (Lally et al. 2014). Increases in local gamma power are also associated with successful memory encoding in humans (Burke et al. 2013). Likewise, in rats, fear conditioning increases gamma synchronization in sensory cortex (Headley \& Pare 2013). Increased gamma power predicts retention of tone-shock associations and enhanced representations of the tone associated with shock in the primary auditory cortex (Headley \& Weinberger 2011).

Recent research indicates that $\beta$-adrenoreceptors recruit in-phase oscillations with gamma activity, whereas $\alpha_{1}$-adrenoreceptors recruit out-of-phase oscillations (Haggerty et al. 2013). Given the higher threshold for activating $\beta$-adrenergic than $\alpha_{1}$-adrenergic receptors (see sect. 5.1), 
these results suggest that high NE levels at hotspots engage $\beta$-adrenoreceptors, recruit in-phase oscillations, and increase local network connectivity for prioritized representations. Elsewhere, lower NE levels should only be sufficient to engage $\alpha_{1}$-adrenoreceptors and thereby reduce local gamma power and diminish local synchronization.

In addition to modulating oscillations in local neuronal ensembles, NE also facilitates oscillatory coupling across regions. Current frameworks of neural synchrony posit that long-range/interregional communication between areas is modulated by oscillation in low-frequency bands, such as theta (4-8 Hz), whereas communication within local networks is modulated by high frequencies, including gamma synchrony (Canolty \& Knight 2010; Von Stein \& Sarnthein 2000). New research further suggests that optimal network function occurs when gamma is embedded in, and phasically facilitated by, slower theta (or even delta [Lakatos et al. 2008]) oscillations (Canolty \& Knight 2010; but see Burke et al. 2013). This theta-gamma coupling seems to provide a mechanism for interregional communication and cross-location phase coupling across regions to help translate local NE hotspots into global effects.

Activation of the LC-NE system promotes hippocampal theta (e.g., Berridge \& Foote 1991; Walling et al. 2011) and is linked to enhancement of novelty-related hippocampal theta (Kocsis et al. 2007). In humans, the phase coupling of gamma with slower oscillations has been described primarily for neocortex (Canolty et al. 2006), where the role of LC-NE in slower rhythms is less well studied. However, hippocampal theta entrains prefrontal cortical theta (Paz et al. 2008). Recently, selective LC-NE activation was found to increase neocortical theta in anesthetized animals (Vazey \& Aston-Jones 2014). The parvalbumin neurons modulated by $\mathrm{NE}$ participate in setting not only gamma, but also theta rhythms (Varga et al. 2014; Wulff et al. 2009); thus, parvalbumin interneurons provide a mechanism for LC-NE support of phase-coupled rhythms. Indeed, lesions of NMDA receptors in the parvalbumin neurons result in decreased power of theta oscillations and reduced modulations of gamma oscillation by theta (Korotkova et al. 2010). NE modulation of the hyperpolarization-associated Ih current has also been proposed to support thalamocortical driving of slower neocortical oscillations (Yue \& Huguenard 2001). Thus, by modulating gamma and theta, the LC-NE system can amplify the winner-take-more effects of hotspots.

\subsection{Key brain regions help evaluate priority and modulate NE hotspots}

Here we review how several key brain regions help enhance GANE selectivity mechanisms under arousal. These regions help detect saliency and interact with the LC to fine-tune priority signals via their own hotspot-like effects (e.g., amygdala) and/or other NE mechanisms (e.g., prefrontal cortex and thalamus).

The amygdala plays a central role in enhancing selectivity under arousal. It helps notice and track salient information (Sander et al. 2003) and recruits the LC when activated (e.g., Bouret et al. 2003; Fallon et al. 1978; Jones \& Moore 1977; Price \& Amaral 1981; Van Bockstaele et al. 1998). The LC, in turn, modulates amygdala activity via $\mathrm{NE}$ to further enhance the saliency signal (Sears et al.
2013). Through its strong anatomical projections to sensory cortices (Amaral et al. 2003), the amygdala amplifies cortical processing of behaviorally relevant events (Chau \& Galvez 2012; Pessoa \& Adolphs 2010). Such modulation of other regions may be mediated by amplification of saliency signals by glutamate-NE interactions within the amygdala (Fig. 7) (see Liu et al. 2009), thereby enhancing the amygdala's selective modulatory influence on other regions. In addition, as reviewed previously (see sect. 3.2), $\beta$-adrenoreceptors in the amygdala mediate the selective effects of arousal on memory.

The thalamus helps control the communication of sensory information across the brain (Sherman 2005). Within the thalamus, there are dense NE fibers and high levels of NE in the pulvinar posteriolateral/posteriomedial complex, but very few in the lateral geniculate nucleus (Morrison \& Foote 1986; Oke et al. 1978). Through its widespread reciprocal connections with cortical and subcortical structures (Shipp 2003), the pulvinar helps filter inputs based on behavioral relevance (Fischer \& Whitney 2012), promotes communication across brain regions (Saalmann \& Kastner 2009; Saalmann et al. 2012), modulates gamma oscillations (Shumikhina \& Molotchnikoff 1999), and controls the gain of sensory processing (Purushothaman et al. 2012). In addition, the pulvinar is sensitive to emotional saliency (Liddell et al. 2005; Padmala et al. 2010; Troiani \& Schultz 2013). Thus, anatomically, NE is set up to modulate thalamic signals of priority.

Furthermore, in rats, NE increases signal-to-noise processing within the thalamus. When directly infused with $\mathrm{NE}$, rat ventral posteriomedial thalamus exhibits reduced spontaneous firing, but enhanced firing in response to whisker stimulation (Hirata et al. 2006). When stimulated by phasic or tonic LC activation, ventral posteriomedial thalamus also exhibited increased firing in response to whisker stimulation (Devilbiss \& Waterhouse 2011). However, an intriguing observation was that in sensory barrel field cortex, phasic stimulation of LC enhanced firing to strong whisker stimulation, but slightly impaired firing to weak whisker stimulation, an outcome consistent with the NE hotspot model. This differential response based on stimulus intensity did not, however, occur within the ventral posteriomedial thalamus, where both strong and weak sensory inputs increased firing (Devilbiss \& Waterhouse 2011). This initial finding suggests that $\mathrm{NE}$ influences in sensory thalamus may occur through mechanisms other than NE hotspots. Thus, further work is needed to examine NE's modulatory role in the thalamus. In any case, the thalamus plays a key role in amplifying selectivity under arousal by coordinating responses to salient stimuli across the brain. Such local representations of salient stimuli are then subject to $\mathrm{NE}$ modulatory influences.

The prefrontal cortex (PFC), including the orbital frontal cortex (OFC) and anterior cingulate cortex (ACC), has reciprocal connections with the LC (Arnsten \& GoldmanRakic 1984; Jodo et al. 1998) and is an important regulator of LC output. PFC regions help appraise sensory information and recruit the LC based on goal relevance (AstonJones \& Cohen 2005), motivational relevance (Mohanty et al. 2008), reward (for the OFC; Schoenbaum \& Roesch 2005), conflict (Botvinick et al. 1999; Sheth et al. 2012), monetary loss (Gehring \& Willoughby 2002), and pain (Rainville et al. 1997). The ACC is also a key site for 
integrating task-relevant and arousal inputs (Pessoa 2009; Shackman et al. 2011). In humans, LC innervation of the PFC is relatively sparse, especially in anterior regions (Gaspar et al. 1989; Javoy-Agid et al. 1989), but NE modulates working memory processes in PFC (Arnsten 2011; Wang et al. 2007).

These PFC noradrenergic influences on working memory have different mechanisms than the NE hotspot. First, in our model, $\beta$-adrenoreceptors support positive feedback loops at NE-glutamate hotspots, but $\alpha_{2}$-adrenoreceptors suppress those feedback loops (see sect. 5.1). However, the facilitatory versus inhibitory role of these adrenoreceptors reverses in the context of working memory. $\beta$-Adrenoreceptors stimulate cAMP, whereas $\alpha_{2}$-adrenoreceptors inhibit it (Duman \& Enna 1986; Nomura et al. 2014; Robinson \& Siegelbaum 2003). Inhibition of cAMP via stimulation of postsynaptic $\alpha_{2}$-adrenoreceptors increases input resistance and enhances recurrent network activity and working memory performance (Wang et al. 2007). Thus, by activating via $\alpha_{2}$-adrenoreceptors, moderate levels of arousal should enhance working memory processes that maintain goal-relevant information in mind, whereas by activating $\beta$-adrenoreceptors, high levels of arousal should impair these processes (Arnsten 2011; Kuhbandner \& Zehetleitner 2011). Such impairments may, in turn, disrupt initiation of top-down prioritization goals after exposure to emotionally salient stimuli (Sutherland et al., in press).

One interesting question is what might occur when top-down priority and bottom-up priority conflict. The insula plays a key role in this aspect and integrates salience signals from internal and external stimuli (Craig 2009; Uddin 2015). The insula is involved in various types of saliency processing, including error detection (Ullsperger et al. 2010), interoception (Craig 2009), oddball detection (Harsay et al. 2012), aversive memory (Miranda \& McGaugh 2004), and detection of events that require cognitive resources (Cai et al. 2015). Although not much is known about LC-insula interactions, the LC and other NE brainstem sites project to the insula (at least in rats) Robertson et al. 2013). Neuroimaging studies also suggest that elevated LC-NE activity is associated with encoding-related activity in the insula in response to aversive stimuli (Clewett et al. 2014; Rasch et al. 2009). Consistent with GANE, motivated (higher-priority) versus passive viewing of emotional faces enhances functional connectivity within face processing networks, including the insula and LC (Skelly \& Decety 2012; but see Astafiev et al. 2010 for caution when interpreting results from LC fMRI).

\subsection{NE amplifies activity in behaviorally relevant functional brain networks}

Along with the dorsal ACC, the insula is a key node in a broader "salience network" (Eckert et al. 2009; Hermans et al. 2011) that helps integrate different sources of saliency (Seeley et al. 2007), guide adaptive behavior (Bressler \& Menon 2010; Cocchi et al. 2013), and regulate shifts from rest to task-oriented behavior (Sidlauskaite et al. 2014). On the basis of these findings, recent models of the salience network propose that it mediates competitive interactions between antagonistic attention networks that prioritize internal versus external stimuli (Bressler \&
Menon 2010; Menon \& Uddin 2010). Current data suggest that the LC-NE system modulates salience network activity. For example, $\beta$-adrenoreceptor blockade during stress reduces salience network activity (Hermans et al. 2011), and salience network activity is associated with pupil and autonomic responses to errors (Critchley et al. 2005) and overall arousal (Sadaghiani \& D'Esposito 2014). In neuroimaging studies, the LC co-activates with the dorsal anterior cingulate during the detection of novel stimuli (Krebs et al. 2013) and during task switching (von der Gablentz et al. 2015), a proposed function of the salience network.

Anatomically, activation of the LC-NE system is well positioned to modulate activity based on priority, as some of the most dense NE innervation is to frontoparietal regions (Gaspar et al. 1989; Javoy-Agid et al. 1989; Morrison \& Foote 1986) that coordinate attention to salient stimuli via priority maps (Ptak 2012). Indeed, phasic LC responses, as indexed by pupil dilation, correlate with activity in a dorsal frontoparietal network during focused attention (Alnæs et al. 2014). However, more generally, according to the GANE model, activation of the LC-NE system should amplify activity in whichever functional network is currently dominant. Consistent with a role for $\mathrm{NE}$ in mediating this process, while subjects rest, pupil dilation increases as activity in the functional network associated with resting state activity increases and activity in a competing motor network activity is suppressed (Yellin et al. 2015). In addition, NE preferentially enhances ventral frontoparietal attention network activity during the detection of salient events that trigger re-orienting (Corbetta et al. 2008; Strange $\&$ Dolan 2007). Thus, NE's influence on gain modulation also manifests at the whole-brain level.

\subsection{Summary}

Arousal's dual effects on cognition pervade multilevel brain systems to amplify the priority of important information. By modulating theta and gamma oscillations, NE preferentially synchronizes activity between high-glutamate regions, leading to "winner-take-more" effects in perception and memory. Like some earlier emotion-cognition theories (e.g., Pessoa \& Adolphs 2010), the GANE model favors the perspective that the amygdala coordinates information transfer within broader networks that influence salience processing and is not the only route by which NE enhances processing of prioritized stimuli. Brain regions that evaluate saliency modulate LC activity either directly via afferent inputs or indirectly via broader networks. Without contextual signals from these central structures and the periphery, the LC would be blind to salient events that demand attention (Sara \& Bouret 2012). In turn, the resulting increase in $\mathrm{NE}$ release activates these modulatory structures to further bias neural processing in favor of high-priority stimuli. On a larger scale, NE modulates activity in a salience network that mediates competitive interactions between frontoparietal attention networks supporting higher-level representations of priority. Thus, according to the GANE model, reciprocal interactions between the LC and hierarchical brain networks help strengthen and reinforce priority-biasing signals under phasic arousal (see Fig. 8). 
Glutamate Amplifies Noradrenergic Effects (GANE) Model
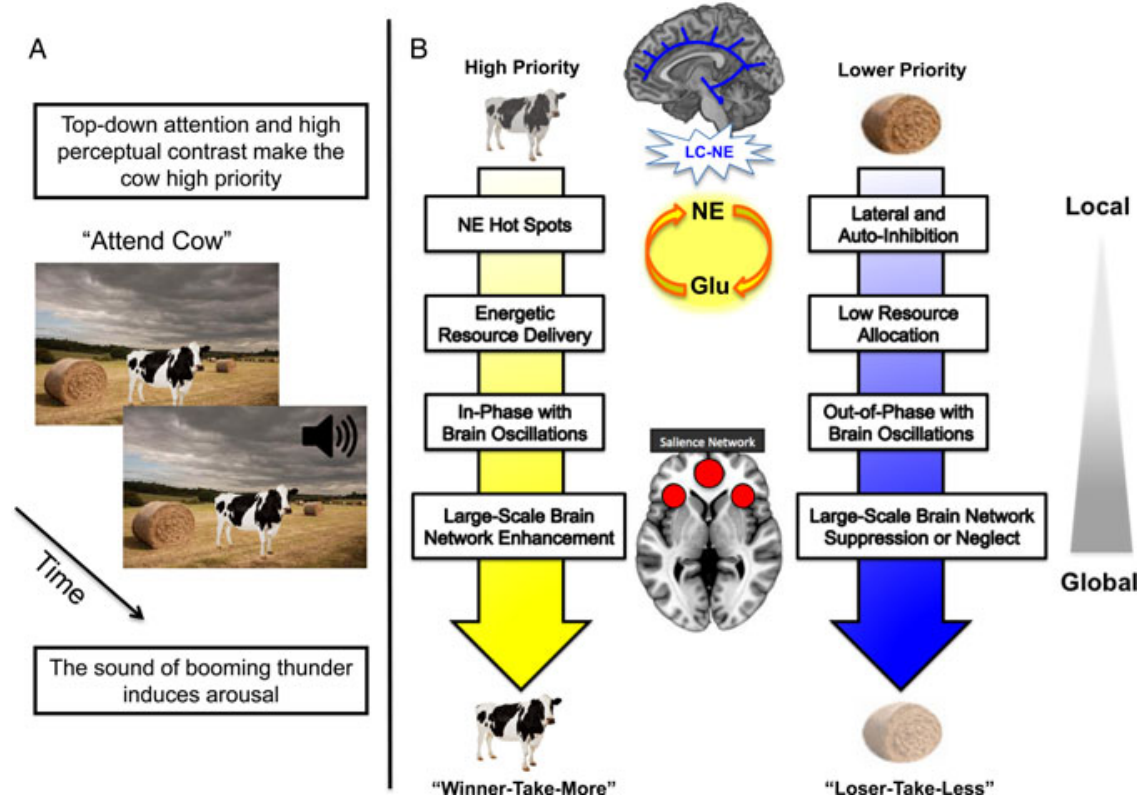

Figure 8. Summary of the glutamate amplifies noradrenergic effects (GANE) model. (A) An example of how arousal biases perception and memory to favor prioritized information. High perceptual contrast (bottom-up) and top-down attention prioritize processing of the cow stimulus in the brain over a less salient hay bale. The sound of booming thunder induces arousal and triggers phasic norepinephrine (NE) release. (B) Salience-evaluating structures, such as the amygdala and prefrontal cortex, recruit locus coeruleus (LC) firing to enable $\mathrm{NE}$ to modulate ongoing processing at multiple levels of brain function. In the high-priority processing pathway, NE interacts with high local glutamate levels to create "hotspots" that increase the "cow" representational activity even further. These local hotspots recruit energetic resources, synchronize oscillations, lead to enhanced activity in high priority large-scale networks, and increase synaptic plasticity. Local glutamate-NE effects occur parallel to more broad-scale suppression, as NE recruits lateral and auto-inhibitory processes that suppress weaker glutamate signals in lower-priority processing pathways. Together, these noradrenergic mechanisms lead to "winner-take-more" and "loser-take-less" outcomes in perception and memory under arousal, such that the cow is even more likely to be remembered, whereas the hay bale is even more likely to be forgotten. ACC = anterior cingulate cortex; INS = insular cortex.

\section{Existing models of LC modulation of cognition}

In this section, we discuss how the GANE model relates to existing theories of LC neuromodulation of cognition that we have not already discussed.

\subsection{Adaptive gain theory}

The adaptive gain theory (Aston-Jones \& Cohen 2005) posits that two different modes of LC activity (phasic vs. tonic) adaptively adjust the gain of cortical information processing to optimize behavioral performance. Phasic LC activity serves as a temporal attentional filter to selectively process task-relevant stimuli and filter out task-irrelevant stimuli, whereas tonic LC activity regulates overall arousal level in the brain. Phasic LC responses to target detection are constrained by background LC activity and occur most frequently during moderate levels of tonic activity (Usher et al. 1999). Adaptive gain theory provides predictions similar to those of the GANE model in terms of the role of the phasic LC mode: phasic LC activity should increase the gain of task-relevant inputs over noisy or task-irrelevant activity. Our GANE model provides a neuromechanism for these effects by proposing that low to moderate $\mathrm{NE}$ levels create ideal conditions to ignite and sustain local NE hotspots via greater phasic LC responses. In support of this notion, a recent fMRI study used baseline pupil dilation before trials of a reward-learning task as a measure of tonic LC-NE activity (Eldar et al.
2013). Both low baseline pupil diameter before the trial and high pupil dilation response during the trial were associated with stronger brain activation in response to task-relevant, but not task-irrelevant stimuli.

\subsection{Network reset theory}

The LC-NE system activates in response to various salient stimuli, including novel, uncertain, or emotionally salient stimuli (Sara 2009; Yu \& Dayan 2005). The network reset theory proposes that when these stimuli are detected, the LC issues a phasic "reset" signal that reorganizes neural networks to facilitate behavioral and cognitive shifts accordingly (Bouret \& Sara 2005; Sara \& Bouret 2012). This theory explains why emotionally salient stimuli and the sudden onset of goal-relevant or perceptually salient stimuli are preferentially perceived and remembered: these events activate the $\mathrm{LC}$, which then reconfigures functional brain networks to process new sources of priority while impairing ongoing processing of other stimuli. This model, however, does not offer a clear explanation of why phasic arousal induced when encountering emotional stimuli can enhance processing of preceding stimuli when they have high priority.

To explain both the facilitative and impairing effects of emotional arousal on preceding stimuli, the GANE model posits that the incidental release of NE by something emotional can instead maintain-or even enhance-ongoing functional network connectivity when those networks are 
Mather et al.: Norepinephrine ignites local hotspots of neuronal excitation

highly activated. Stimulating the LC can inhibit feedforward inhibition by interneurons, thereby increasing the throughput of coincident sensory (glutamatergic) inputs (Brown et al. 2005). Although this "loosening" of neurotransmission enables network flexibility and the building of new representations, the GANE model's prediction that strong glutamatergic signals transmitting a prioritized representation will benefit from sudden LC activation explains how the "reset" signal triggered by phasic LC activity can still enhance processing of preceding high-priority stimuli.

\subsection{Summary}

The GANE model both complements and extends previous models of how cognition is influenced by the LC-NE system. According to adaptive gain theory, high phasic LC activity promotes exploitation of the current focus of attention over exploration of other options. In contrast, the network reset theory proposes that phasic LC activity promotes a global reset of attention. The GANE model reconciles these two theories by highlighting the role of priority. According to the GANE model, if the current focus of attention has sufficient priority to yield high glutamate release in synapses transmitting those stimuli, then a phasic increase in LC activity should enhance processing of those representations. Otherwise, increases in LC activity should shift attention and neural resource allocation towards new sources of priority.

The GANE model extends current models of LC function by positing that under arousal, local glutamate-NE interactions will amplify activity of high-priority representations regardless of how those representations initially became highly active. Thus, although the GANE model provides neural mechanisms that account for arousal increasing biased competition outcomes, it can also accommodate other models or modes of information prioritization (Friston 2010; Keitel et al. 2013; Reynolds \& Heeger 2009; Wieser et al. 2011).

\section{Potential boundary conditions and questions for future research}

In this article, we have argued that arousal leads to winnertake-more and loser-take-less effects in perception and memory via local and global noradrenergic mechanisms in the brain. Yet, although the GANE model explains many findings observed in the emotion-cognition literature, there are a number of important questions for future research.

Arousal may not increase selectivity as effectively among older adults because of age-related changes in the LC-NE system, including loss of LC neurons (Manaye et al. 1995; Mather \& Harley 2016; Sladek \& Sladek 1978; Vijayashankar \& Brody 1979). Recent autopsy evidence indicates that lower LC neuron density is related to the rate of cognitive decline prior to death, even after controlling for decline in other aminergic nuclei (e.g., dorsal raphe, ventral tegmental area) (Wilson et al. 2013). $\beta$ - and $\alpha_{2}$-adrenoreceptors may also be affected in aging (e.g., Bigham \& Lidow 1995; Kalaria et al. 1989). Decreases in $\alpha_{2}$-adrenoreceptor activity may contribute to age-related cognitive declines because agonists that engage $\alpha_{2 \mathrm{~A}}$-adrenoreceptors can improve age-related deficits in working memory (Arnsten \& Cai 1993; Arnsten \& Goldman-Rakic 1985; Ramos et al. 2006), potentially via $\alpha_{2 \mathrm{~A}}$-induced improvements in the ability to maintain focused attention (Decamp et al. 2011). Aging also affects how effectively glutamate triggers additional NE release (Gonzales et al. 1991; Pittaluga et al. 1993), which would disrupt the emergence and/or efficacy of NE hotspots in older adults.

Another question involves sleep, which plays a crucial role in selectively consolidating salient memory traces (Diekelmann \& Born 2010), including emotional stimuli (Hu et al. 2006; Payne et al. 2008; 2012) and top-down prioritized information (Rauchs et al. 2011; Saletin et al. 2011). Emerging research suggests that the LC-NE system may enhance memory consolidation during slow wave sleep (non-rapid eye movement [NREM]), a period when high-priority neural ensembles reactivate (for a review, see Dang-Vu et al. 2008; Eschenko et al. 2012; Sara 2010). For example, a learning-dependent increase in LC activity occurs during slow wave sleep (Eschenko \& Sara 2008), and depleting NE prior to encoding reduces slow wave sleep that night (Cirelli et al. 2005). Pharmacologically enhancing LC-NE system activity during slow wave sleep improves recognition of odors learned within the previous 3 hours, whereas blocking LC-NE activity impairs odor recognition (Gais et al. 2011). Blocking NE during sleep also leads to greater memory impairment for emotional than for neutral stimuli (Groch et al. 2011). The timing of transient LC activity coincides with the slow wave grouping of hippocampal sharp wave ripple complexes and sleep spindles that promote NMDA-mediated cellular plasticity (Diekelmann \& Born 2010; Rosanova \& Ulrich 2005). NE may interact with these processes, given evidence that pharmacological activation of $\beta$-adrenoreceptors facilitates the emergence of sharp waves and the induction of LTP (Ul Haq et al. 2012). Together these findings raise the intriguing possibility that the precise timing of $\mathrm{NE}$ release interacts with the reactivation of high-priority memory networks to facilitate GANE effects during slow wave sleep.

In this article, we focused on perception, encoding, and consolidation processes, but another important question for future research is how NE modulates memory retrieval (e.g., Sterpenich et al. 2006). For example, when encountering a new experience, our memory system can either store this novel information as a distinct memory (i.e., requiring pattern separation) or use it to reactivate existing memories (i.e., requiring pattern completion) (Bakker et al. 2008). Previous research indicated that arousal facilitates pattern separation (Segal et al. 2012) and that NE facilitates retrieval or pattern completion (Devauges \& Sara 1991). But it has been unclear how NE/arousal modulates competition between these two hippocampal processing modes. Glutamate amplification of noradrenergic effects might also affect the stability of a salient memory after it is retrieved, or reconsolidated, because this process involves $\beta$-adrenoreceptor and NMDA receptor activation (Lee et al. 2006; Przybyslawski et al. 1999).

Another open question concerns the timing of these effects. Behavioral data indicate that presenting an emotionally salient item influences memory of items appearing in the past few seconds (e.g., Sakaki et al. 2014a) and memory of items appearing in the next few seconds, as well (e.g., Sutherland \& Mather 2012). It is plausible that the phasic release of $\mathrm{NE}$ would have effects on this time scale, but research examining NE-glutamate interactions is needed to address this question. 
Commentary/Mather et al.: Norepinephrine ignites local hotspots of neuronal excitation

On the tonic side of the equation, events that induce stress activate both the LC-NE system and the hypothalamic pituitary adrenal (HPA) axis (Pacak \& Palkovits 2001; Sved et al. 2002), and these two systems interact in many ways, especially via the actions of corticotropin releasing factor $(\mathrm{CRF})$. Released by the hypothalamus under stress, CRF helps to initiate the HPA axis response while also targeting the LC (Carrasco \& Van de Kar 2003; Valentino \& Van Bockstaele 2001; Van Bockstaele et al. 2001). CRF influences both tonic LC activity and sensory-evoked phasic discharge, either enhancing or impairing sensory-evoked phasic responses depending on waking state and CRF levels administered (Bangasser \& Valentino 2012; Devilbiss et al. 2012; Zitnik et al. 2014). One possibility is that by modulating tonic levels of LC activity, stress also enhances or constrains the impact of phasic arousal responses (see sect. 8.1).

Human genetic studies suggest that different NE polymorphisms moderate the strength of arousal's influence on memory and perceptual processing. To date, much of this research has focused on the ADRA2B deletion variant in which there is reduced NE inhibitory signaling. In human ADRA2B deletion carriers, there is greater activity in the amygdala and insula during the viewing or encoding of emotional versus neutral images (Cousijn et al. 2010; Rasch et al. 2009). Such patterns of NE-related activity are believed to underlie the larger advantage of emotionally salient over neutral stimuli in memory (de Quervain et al. 2007) and perception (Todd et al. 2011; 2013) observed in these individuals.

It is, however, unclear how these genetic effects relate to the NE hotspot mechanisms outlined in the GANE model. Whereas $\alpha_{2 \mathrm{~A}}$-adrenoreceptors are found throughout much of the brain and have been clearly identified as autoreceptors regulating $\mathrm{NE}$ release, the $\alpha_{2 \mathrm{~B}}$-adrenoreceptors associated with this genetic polymorphism have a different profile (Brede et al. 2004). They are most dense in striatum, globus pallidus, and thalamus (De Vos et al. 1992; Saunders \& Limbird 1999) and are essential for regulating the fetal blood supply (Brede et al. 2004). Thus, although it is possible that these genetic effects alter the feedback cycle in NE hotspots, the genetic differences could also be mediated by different developmental pathways, thalamic modulation of emotional input, or some other factor.

Related to this point about the differential brain localization of $\alpha_{2 B}$-adrenoreceptors is the more general question of how regional variation in receptor density (e.g., Zilles \& Amunts 2009) modulates hotspot effects. Modeling and direct comparisons of $\mathrm{NE}$-glutamate interactions across regions could help address this question. In addition, although we have focused on how the LC-NE system influences cognition, other neuromodulators such as serotonin, dopamine, and acetylcholine share many mechanisms of action with NE (Hurley et al. 2004) and interact with NE to regulate attention, memory, and arousal (Arnsten 2011; Briand et al. 2007; Sara 2009). Such interactions are likely to modulate the NE-glutamate interactions highlighted here (some examples already described in sect. 5.1 are interactions with orexin, histamine, glycine, and serine). These interactions may allow for more nuanced effects and some redundancy within the arousal system. However, given NE's core role in arousal and broad innervation of much of the brain, including source nuclei of other neuromodulators (e.g., ventral tegmental area and basal forebrain) (Jones 2004; Sara 2009), we expect that it plays the lead role in modulating cognitive selectivity as arousal levels fluctuate.

\section{Conclusion}

Selection is at the core of what allows our cognitive systems to function effectively, enabling us to process the constant influx of information and retrieve the experiences most relevant for adaptive behavior and maintenance of well-being. The ability to focus on salient information is especially important during situations that induce arousal, such as during exposure to threatening or exciting sounds or objects and the pressure to perform a challenging task. For more than 50 years, there has been robust behavioral evidence that arousal often simultaneously enhances and impairs processing of different types of neutral information (Easterbrook 1959). Yet brain-based accounts of how arousal influences cognition failed to address how such dual effects could arise.

Our GANE model fills this critical gap. In this framework, we propose that increases in $\mathrm{NE}$ levels under arousal enhance the selectivity of information processing. GANE builds on the previous ABC model (Mather \& Sutherland 2011) to provide neural mechanisms of how $\mathrm{NE}$ leads to winner-take-more and loser-take-less effects in perception, attention, and memory. Unlike the $\mathrm{ABC}$ model, however, the GANE model does not require competition to be a fundamental mechanism. Instead, the GANE model selectively amplifies the activity of whatever priority mechanisms are operating.

Under phasic arousal, local glutamate signals corresponding to a highly activated percept interact with NE to create a hotspot of even higher levels of activity, whereas lowerpriority representations are either neglected or further suppressed. These self-regulating hotspots are further aided by NE's recruitment of brain structures and large-scale functional networks that determine which stimuli deserve attention. NE directs blood flow and energetic resources to brain regions transmitting prioritized information. It supports selective memory consolidation via initiation of LTP and LTD. Through all of these processes, NE increases the gain of prioritized information in the brain, such that things that matter stand out even more and are remembered even better, while the mundane and irrelevant recede even farther into the background and are ignored or forgotten.

\section{Open Peer Commentary}

\section{Glutamate and norepinephrine interaction: Relevance to higher cognitive operations and psychopathology}

\author{
doi:10.1017/S0140525X15001727, e201
}


Commentary/Mather et al.: Norepinephrine ignites local hotspots of neuronal excitation

\section{chadi.abdallah@yale.edu_lynnette.averill@yale.edu \\ john.krystal@yale.edu steven.southwick@yale.edu}

amy.arnsten@yale.edu

Abstract: Mather and colleagues present an impressive interdisciplinary model of arousal-induced norepinephrine release and its role in selectively enhancing/inhibiting perception, attention, and memory consolidation. This model will require empirical investigation to test its validity and generalizability beyond classic norepinephrine circuits because it simplifies extremely complex and heterogeneous actions including norepinephrine mechanisms related to higher cognitive circuits and psychopathology.

In their target article, Mather and colleagues propose a molecular model, glutamate amplifies noradrenergic effects (GANE), through which arousal enhances or inhibits perception, attention, and memory consolidation. In this model, arousal precipitates phasic release of norepinephrine (NE) throughout the brain, but "hotspots" of NE release are generated near activated glutamate circuits, sufficient to engage low-affinity $\beta$-adrenoceptors, which further increase glutamate release and enhance postsynaptic plasticity by increasing cAMP signaling. The model provides an impressive integration across several fields but will require empirical investigation to test its validity. Furthermore, although the model is presented as universally applicable throughout the brain, NE actually has very heterogeneous actions in different brain circuits. In particular, although the GANE model addresses the effects of normal arousal mechanisms in sensory cortex and hippocampus, it is important to discuss how this model may relate to $\mathrm{NE}$ actions in higher cognitive circuits and to conditions of psychopathology.

The noradrenergic system plays an essential role in the pathophysiology and treatment of psychiatric disorders. For example, noradrenergic dysregulation is associated with post-traumatic stress disorder (PTSD), and $\alpha_{1}$-antagonists can reduce these symptoms (Arnsten et al. 2015b; Southwick et al. 1999). Many antidepressants target the noradrenergic system (Klimek et al. 1997), and $\alpha_{2 \mathrm{~A}}$-agonists enhance cognition in patients with attention deficit hyperactivity disorder (ADHD) (Arnsten \& Wang 2016). Similarly, accumulating evidence implicates glutamate in the etiology and treatment of mental disorders (Chambers et al. 1999; Krystal et al. 2013). Whether the GANE model applies to traumatic stress conditions is not clear; the research Mather et al. cite utilized subtle arousing conditions - for example, an emotional word. It is, however, likely to explain several aspects of PTSD: for example, enhancement of the consolidation of traumatic events that may contribute to flashbacks and intrusive memories. However, additional, higher brain changes during trauma may not be captured by this model, as NE actions in the brain are more heterogeneous than described.

Most important for human cognition, the newly evolved circuits in layer III of the dorsolateral prefrontal cortex (dlPFC) that underlie higher cognitive operations are modulated in a unique manner that is often opposite that of classic synapses in sensory cortex, amygdala, and hippocampus (Arnsten et al. 2012). Indeed, these newly evolved "delay cell" circuits in the dIPFC are even regulated differently than sensory/response-related neurons within the dIPFC. For example, delay cell persistent firing is mediated by NMDAR with NR2B subunits that are exclusively in the postsynaptic density, not extrasynaptic as they are in classic synapses (Wang et al. 2013). Furthermore, delay cells are only subtly influenced by AMPA receptors and show reduced, rather than increased, neuronal firing following systemic ketamine (Wang et al. 2013). In contrast, response feedback cells in the dIPFC (likely layer V) have a more classic profile, with large AMPA receptor influences and increased firing with systemic ketamine (Wang et al. 2013). These marked differences extend to intracellular cAMP signaling events as well. In classic synapses, activation of cAMP signaling, for example, arising from $\beta$-adrenoceptor stimulation, increases glutamate release from axon terminals and strengthens long-term potentiation (LTP) postsynaptically. However, in layer III dlPFC circuits, increased cAMP signaling weakens connections by opening cAMP-PKA-regulated potassium channels in dendritic spines (Arnsten 2015; Arnsten et al. 2012). Instead, it is inhibition of cAMP signaling via postsynaptic $\alpha_{2 \mathrm{~A}}$-adrenoceptors that strengthens network connectivity by closing potassium channels near the synapse (Wang et al. 2007). There is currently no evidence of NE "hotspots" in these circuits; for example, blockade of $\beta$-receptors within the primate dlPFC has no effect on working memory performance (Li \& Mei 1994), even though there are likely high levels of glutamate release in dlPFC arising from the persistent firing of these neuronal networks. Thus, the model in Figure 6 of the target article is misleading because it does not differentiate $\mathrm{NE}$ actions in classic synapses from those in more newly evolved dlPFC circuits.

Mather et al. also provide an oversimplified discussion of $\mathrm{NE}$ actions at $\alpha_{1}$-adrenoceptors. Although they focus on $\alpha_{1}$ mechanisms that weaken plasticity, $\alpha_{1}$ promotes synaptic actions in many synapses - for example, in somatosensory cortex (Mouradian et al. 1991; Waterhouse et al. 1981; 2000). There are also key circuits where $\alpha_{1}$-receptor activation potentiates $\beta$-receptor actions: For example, in amygdala, $\alpha_{1}$-receptors facilitate $\beta$-adrenergic enhancement of memory consolidation (Ferry et al. 1999a; 1999b). These effects are opposite those described by Mather and colleagues. Their model also does not capture the important finding that high levels of NE release in PFC during stress decrease persistent firing and working memory abilities through stimulation of $\alpha_{1}$ receptors (Birnbaum et al. 2004). All of these actions likely have a key effect in switching control of behavior from thoughtful, flexible, top-down control by PFC under conditions of safety (moderate levels of arousal) to reflexive, unconscious habits mediated by sensorimotor cortex and subcortical structures during uncontrollable stress (very high levels of arousal).

These mechanisms have particular relevance to the symptoms of PTSD, for which there is extensive evidence of elevated noradrenergic activity (Southwick et al. 1999). For example, the $\alpha_{2}$-antagonist yohimbine worsens symptoms and induces hypofrontality in subjects with PTSD at doses that have little effect in control subjects (Bremner et al. 1997; Southwick et al. 1993). These drug actions may arise from a combination of neural events, for example, loss of dIPFC top-down control from blockade of postsynaptic $\alpha_{2 \mathrm{~A}}$-receptors and increased NE stimulation of $\alpha_{1}$-receptors in dIPFC, as well as increased NE release in "hotspots" in the amygdala, hippocampus, and sensory cortex that may exacerbate anxiety and flashbacks (Arnsten et al. 2015b). Thus, the GANE model may apply to NE actions in classic brain circuits, but not to those in higher cortical circuits, which are strengthened by $\alpha_{2 \mathrm{~A}^{-}}$rather than $\beta$-adrenoceptor mechanisms.

\section{Why we forget our dreams: Acetylcholine and norepinephrine in wakefulness and REM sleep}

doi:10.1017/S0140525X15001739, e202

\section{Andrea Becchetti ${ }^{\mathrm{a}}$ and Alida Amadeo ${ }^{\mathrm{b}}$ \\ ${ }^{a}$ Department of Biotechnology and Biosciences and Center for Neuroscience of Milan, University of Milano-Bicocca, 20128 Milan, Italy; ${ }^{\mathrm{b}}$ Department of Biosciences, University of Milano, 20133 Milan, Italy. \\ andrea.becchetti@unimib.it \\ http://www.btbs.unimib.it \\ alida.amadeo@unimi.it \\ http://www.dbs.unimi.it}


Regulation of neocortical circuits by ascending regulatory systems involves all of the classic neurotransmitters. Most of the nuclei located in the brainstem, hypothalamus, and basal forebrain not only are reciprocally connected, but also send direct projections to the neocortex (Jones 2011; Saper et al. 2010; Steriade \& McCarley 2005). The same applies to release by the hypothalamic nuclei of neuropeptides such as orexin/hypocretin in wakefulness and melanin-concentrating hormone $(\mathrm{MCH})$ in rapid-eye-movement (REM) sleep (Aracri et al. 2015; Jones \& Hassani 2008; Monti et al. 2013; and references therein). As a first approximation, these bewildering intricacies can be simplified by focusing on the balance in activity between noradrenergic and cholinergic nuclei, which are crucial regulators of arousal and cognition (e.g., Constantinople \& Bruno 2011; Schmidt et al. 2013). Both project varicose fibers that widely innervate the neocortex, and their global effects are excitatory. During wakefulness, high levels of norepinephrine $(\mathrm{NE})$ and acetylcholine $(\mathrm{ACh})$ cooperate in regulating arousal and cognitive processes. However, although cholinergic transmission is certainly implicated in synaptic plasticity (e.g., Berg 2011), the physiological action of NE is thought to be more persistent and more closely related to memory retention and consolidation (e.g., Constantinople \& Bruno 2011; McGaugh 2013; Schmidt et al. 2013). The activity of noradrenergic and cholinergic neurons decreases during non-REM (NREM) sleep, whereas in REM sleep, ACh release increases again, whereas NE activity remains low (Datta 2010; Lee et al. 2005; Saper et al. 2010; Takahashi et al. 2010) (Fig. 1). The fact that neocortex activation in REM sleep is sustained mainly by ACh is a further indication that the cholinergic tone is more directly related to consciousness and executive functions. In fact, the role of REM sleep in memory consolidation remains controversial (Ackermann \& Rasch 2014; Rasch \& Born 2013).

Does the GANE model help suggest possible explanations of the different functional consequences of activating these regulatory systems during brain states? A first central assumption is that, under strong neuronal activation, spillover glutamate stimulates nearby $\mathrm{NE}$ varicosities in an $\mathrm{N}$-methyl-D-aspartate (NMDA) receptor-mediated manner. By activating low-affinity $\beta$-adrenoreceptors, high NE release would stimulate neuronal excitability, as well as glutamatergic terminals, thus constituting activity "hotspots" that effectively amplify inputs with high

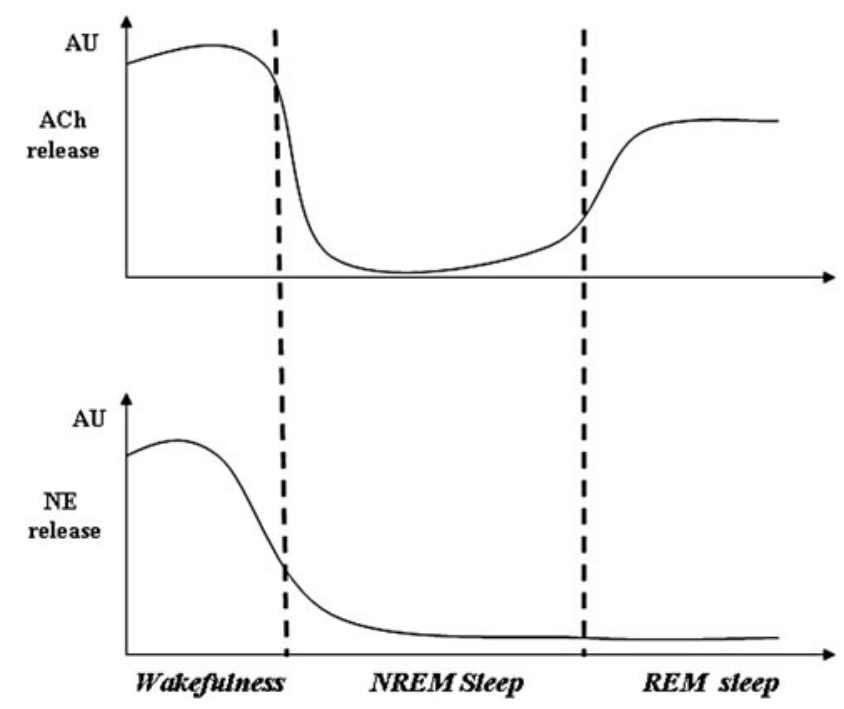

Figure 1 (Becchetti \& Amadeo). Cholinergic and noradrenergic activity through the sleep-wake cycle. The scheme provides a qualitative comparison of the activity of the ascending cholinergic and noradrenergic projections, with no pretension of quantitative precision. AU=arbitrary units. priority under phasic arousal. Are such hotspots possible in the cholinergic system? Not much is known about the glutamatergic regulation of ACh release, but evidence does exist of ionotropic glutamate receptors regulating cholinergic terminals in the neocortex (Ghersi et al. 2003; Parikh et al. 2008). Hence, it is conceivable that spillover glutamate also stimulates cholinergic fibers. Because it is well known that ACh increases glutamate release (Marchi \& Grilli 2010), a positive feedback loop could generate local ACh hotspots, analogous to those hypothesized by Mather and colleagues.

A second tenet of the GANE model is that the low-threshold $\alpha 2$-adrenoreceptors, by responding to low NE concentrations, would inhibit glutamate release in pathways implicated in low priority signaling, under aroused conditions. In this respect, the cholinergic system presents several differences compared with the noradrenergic. In particular: (1) cholinergic fibers form both well-differentiated point-to-point synapses and axon varicosities that sustain diffuse ACh release (Dani \& Bertrand 2007); and (2) ACh activates both metabotropic (muscarinic, mAChRs) and ionotropic (nicotinic, nAChR) receptors. In prefrontal regions, M1 mAChRs are widespread and produce excitatory effects related to working memory through different cellular mechanisms (e.g., McCormick \& Prince 1986; Gulledge et al. 2009; Proulx et al. 2014). Their $\mathrm{EC}_{50}$ for $\mathrm{ACh}$ is in the low $\mu \mathrm{M}$ range. On the other hand, nAChRs can be divided into two functional classes (Dani \& Bertrand 2007). Heteromeric nAChRs have high affinity for $\mathrm{ACh}$ (with $\mathrm{EC}_{50}$ in the $\mu \mathrm{M}$ range), relatively low permeability to $\mathrm{Ca}^{2+}\left(\mathrm{P}_{\mathrm{Ca}}\right)$, and slow desensitization in the presence of agonist. Homomeric nAChRs have high $\mathrm{P}_{\mathrm{Ca}}$ (in the order of the one displayed by NMDA receptors), but low affinity for ACh $\left(\mathrm{EC}_{50} \approx 200 \mu \mathrm{M}\right)$, and quick desensitization kinetics. A striking difference with NE transmission is immediately apparent. The long-term effects on synaptic consolidation are thought to depend on $\mathrm{Ca}^{2+}$ signals. However, within the putative $\mathrm{ACh}$ hotspots, the efficacy of high- $\mathrm{P}_{\mathrm{Ca}}$ homomeric receptors would be blunted by quick desensitization. High ACh concentrations would also tend to desensitize heteromeric nAChRs. This would prevent sustained $\mathrm{Ca}^{2+}$ entry through nAChRs as well as by nAChR-dependent activation of glutamate release, and thus of NMDA receptors. Therefore, it seems unlikely that ACh hotspots can produce long-term cellular effects considerably different from those produced by lower ACh concentrations.

In summary, by following up the GANE model reasoning, one is led to conclude that low and high concentrations of $\mathrm{NE}$ and ACh produce distinct functional effects on neocortical networks. Low to moderate ACh release sustains global neocortex arousal in both wakefulness and REM sleep. However, in the absence of NE activity (as in REM sleep), cholinergic activity is unable to yield long-term synaptic changes, such as those implicated in memory retention, which would partly explain the well-known difficulty of recalling oneiric activity. Instead, high levels of ACh seem more able to shape the rapid synaptic responses implicated in executive functions, as the quick kinetics of the lowaffinity nicotinic ACh receptors would suggest. We believe that deeper functional studies of the interplay between the ascending regulatory systems, led by heuristic models such as GANE, will greatly lead to progress in understanding the physiological basis of cognition.

\section{For better or worse, or for a change?}

doi:10.1017/S0140525X15001740, e203

\section{Sebastien Bouret}

CNRS, Team Motivation Brain \& Behavior, ICM-Institut du Cerveau et de la Moelle épinière, Hôpital Pitié-Salpêtrière, 75013 Paris, France.

sebastien.bouret@icm-institute.org

https://sites.google.com/site/motivationbrainbehavior 
Abstract: The noradrenergic system is intimately related to the autonomic system and is thought to play a key role at the interface between arousal and cognition. The GANE (glutamate amplifies noradrenergic effects) theory proposes a complete account of that role, with an emphasis on the quantitative effect of noradrenaline on stimulus processing. This is in marked contrast to network reset theory, which emphasizes the qualitative effect of noradrenaline of updating the representation of the environment.

Among all neuromodulatory systems, the noradrenergic system is probably the one most closely related to vigilance and autonomic arousal (Aston-Jones et al. 1991; Berridge \& Waterhouse 2003; Carter et al. 2010; Foote et al. 1980; Jacobs 1986; Sara \& Bouret 2012). The activity of locus coeruleus (LC) neurons is so closely related to arousal that autonomic measures such as pupil diameter are used as a proxy for LC activity in human studies (Einhäuser et al. 2008; Jepma \& Nieuwenhuis 2011; Nassar et al. 2012; Preuschoff et al. 2011; Sterpenich et al. 2006; Varazzani et al. 2015). Much less clear, however, are the nature of the influence of LC activation-noradrenaline (NA) release on its targets in the brain and its implication for cognition. The GANE (glutamate amplifies noradrenergic effects) theory developed by Mather et al. is addressing this issue directly. This theory covers numerous aspects of cognition, ranging from attention and decision making to memory and emotions, and proposes an original cellular mechanism.

In line with earlier theories of NA functions, GANE emphasizes the effect of NA on gain, which presumably mediates the inverted-U-shaped relation between the efficacy of sensorimotor functions and arousal (Arnsten 2009; Aston-Jones \& Cohen 2005). Theories such as network reset and unexpected uncertainty are based on a very distinct intuition: The key role of the LC-NA system is to change internal representations, rather than enhance them (Bouret \& Sara 2005; Yu \& Dayan 2005). network reset is based on two features of the NA system: It is extremely well conserved across all vertebrates, and its activation is systematically associated with a profound change in behavior (Bouret \& Sara 2004; Clayton et al. 2004; Dalley et al. 2001; Devauges \& Sara 1990; Jacobs 1986; McGaughy et al. 2008). The typical condition of LC activation is the orienting response to a salient stimulus (Aston-Jones \& Bloom 1981; Bouret \& Sara 2004; Foote et al. 1980). Unexpected uncertainty is based on similar intuitions and emphasizes the role of NA in learning (Yu \& Dayan 2005). Again, there are some differences between neurobiological intuitions proposed in GANE versus network reset, but the key question the authors raise is not "how," but "why": "Why phasic arousal induced when encountering emotional stimuli can enhance processing of preceding stimuli when they have high priority" (sect. 8.2).

That question implies two features: First, the processing of stimuli is taking enough time to allow subsequent emotional stimuli to induce enhancement of this processing via an increase in arousal. This assumption makes strong predictions on the dynamics of these processes, and indeed, such an assumption is important in understanding LC/NA functions. Second, the processing of the original stimulus is not altered qualitatively by the emotional stimulus; it is only enhanced. In other words, the "priority maps" are not modified qualitatively after the onset of this emotional stimulus; arousal is only enhancing their impact on behavior.

The assumptions underlying network reset are different: A salient stimulus would cause a qualitative change in stimulus processing, both the nature of the representation and the associated neuronal activity. The priority maps would be changed. In the extreme version of network rReset, the highest priority would be attributed to the salient stimulus, and the preceding stimulus would be ignored. But if the initial stimulus leaves a trace strong enough to be integrated with the emotional one, after the reset, the new "functional network" would underlie the processing of both stimuli. In that case, the representation of the original stimulus would be modified (changed qualitatively), not quantitatively (enhanced or decreased).
Is the influence of arousing events qualitative (network reset) or quantitative (GANE)? Using the example in Figure 7 of Mather et al., these two theories make radically different predictions: According to network reset, the booming sound of a thunderstorm would not enhance the processing of the cow; it would first trigger an orienting response that consists of interrupting existing activity (including processing of the cow) and promoting redirection of attentional resources. Using the words of Mather et al., the sound would become "high priority," but it would either be processed alone or be combined with the cow in a novel representation. Importantly, the representation of the cow as it existed before the storm would disappear.

Thus, we could rephrase Mather et al.'s question: Why should salient stimulus enhance processing of past events? First, time goes one way only, and modulating past events makes sense only if they are used for the present or for planning future actions (James 1913; Sara 2000). The example provided in Figure 7 is very close to laboratory situations in which discrete stimuli are manipulated in a controlled setting. But imagine yourself walking in the fields, and let's assume that for some reason, you are considering the cow. What will happen if you hear booming thunder? Will you still care about the cow? If yes, what is the chance that you think about it the same way you did before, independently of the critical information provided by the thunder? If the NA system had evolved to enhance the processing of the cow when an inherently more significant stimulus occurs, would this system be so widely represented among living animal species?

In conclusion, in addition to its influence on sensorimotor functions, the LC/NA system has a major role in promoting changes in behavior. The details of the model, including its dynamics, will be critical to understanding how, and why, the release of NA modulates forebrain systems. But this model should account for critical biological features of the LC: It is activated when a behaviorally relevant stimulus triggers a sympathetic response and a behavioral response. For all vertebrates, this autonomic activation is a generic emergency reaction that facilitates coping with a challenge (threat, effort, unexpected event, etc.), and it presumably facilitates the behavioral adjustment to the challenge. This adjustment may take several forms, including gain and/or reset, and be mediated by myriad neurobiological processes, but to understand why the central NA system exists and what it does, it is important to consider ecological problems that the brain has evolved to solve.

\section{The Fluency Amplification Model supports the GANE principle of arousal enhancement}

doi:10.1017/S0140525X15001752, e204

Claus-Christian Carbon ${ }^{\mathrm{a}, \mathrm{b}, \mathrm{c}}$ and Sabine Albrecht ${ }^{\mathrm{a}, \mathrm{b}, \mathrm{c}}$

${ }^{a}$ Department of General Psychology and Methodology, University of Bamberg, Bamberg, Germany; ${ }^{\mathrm{b}}$ Forschungsgruppe EPAEG (Ergonomie, Psychologische AEsthetik, Gestalt), Bamberg, Germany; ${ }^{\mathrm{C} B}$ Bamberg Graduate School of Affective and Cognitive Sciences (BaGrACS), Bamberg, Germany.

ccc@experimental-psychology.com

www.experimental-psychology.com

sabine.albrecht@uni-bamberg.de

Abstract: The GANE (glutamate amplifies noradrenergic effects) model described by Mather et al. offers a neurophysiological basis for the arousal mechanism which is essential for empirical aesthetics and Gestalt processing. More generally, the core principle of perception can be interpreted as a continuous processing of competing arousal states, yielding selective amplification and inhibition of percepts to deduce the meaning of a scene.

The GANE (glutamate amplifies noradrenergic effects) principle Mather et al. describe offers a thorough modeling of how arousal-induced norepinephrine modulates the dynamics of 
information processing. Processing is directed toward high-priority - that is, salient - stimuli, leading to stronger effects on the perception and memory of these stimuli (amplification), whereas the processing of low-priority stimuli is impaired. The recently established fluency amplification model (FAM) (Albrecht \& Carbon 2014), originating from the domain of perceptual and affective sciences, builds on a very compatible mechanism. (Cognitive) fluency refers to the experienced ease with which information is processed, mostly operationalized by processing speed or ease of response generation. Typically, theories on fluency assume that the more fluently a stimulus is processed, the higher the appreciation of this stimulus is. In contrast, FAM interprets fluency in terms of saliency: Fluently processed stimuli are more unambiguous and clearer - they are better representatives of their category. As such, the more fluent the processing of a stimulus the stronger the signal is and, thus, the higher the saliency regarding the stimulus is. In FAM, we propose that this causality leads to an amplification of the original judgments assigned to the stimulus. For example, the assessment of the valence of a fluent stimulus will be an amplified version of the initial stimulus valence: Positive stimuli will be valued even more, and negative stimuli will be devalued in a more intense way when being processed fluently. That effect was exactly what we were able to experimentally confirm for stimuli with positive versus negative valence (Albrecht \& Carbon 2014). Meanwhile, the emotional assessments of stimuli with minimal saliency, in our case stimuli of undetermined valence, were not altered by fluency. Taken together, these results indicate that the saliency of a stimulus, defined as the deviation from the neutral information regarding the target scale, operates as an amplification factor for the base signal, here, the emotional value of the stimulus.

Beyond FAM, the concept of arousal can also be seen as one essential mechanism underlying amplification effects regarding judgment in general (see, e.g., Storbeck \& Clore 2008). The GANE principle offers a plausible neurophysiological basis for a mostly very vaguely defined arousal mechanism that is often used in theories of cognitive sciences. For example, arousal is purported to play an important role in the specific effects of empirical aesthetics (e.g., the misattribution of an internal state-due to unspecified arousal-toward the preference of an object). Arousal is even more influential in the general field of object recognition, where it is assumed to be the key to pooling cognitive resources to increase the probability of solving a perceptual problem, detecting a Gestalt, or recognizing an object. Muth et al. (2015) recently proposed a model explaining the connection between insights (the "aesthetic aha," which goes along with a sudden rise in fluency [Muth \& Carbon 2013]) and preference formation. Aesthetic stimuli are often complex at first sight and difficult to process, which means that they are initially disfluent. Such disfluency indicates the complexity of the perceptual problem, which, in return, signals something potentially meaningful and therefore evokes an orienting reaction plus a state of increased arousal. High arousal shifts attentional and cognitive resources toward the apparent source of complexity, giving rise to interest. Likewise, the GANE model proposes that top-down attention and perceptual features such as contrast and complexity prioritize the processing of certain stimuli over less salient ones. Further elaboration of the stimulus may indeed lead to a decrease in complexity of the visual scene (e.g., by detection of something meaningful or by clear identification of an object), which goes along with a sudden increase in fluency (Albrecht \& Carbon 2014). At the same time, GANE proposes that the processing of salient information is amplified, whereas the processing of less salient information is inhibited. These processes finally result in an insight and the dissolving of arousal.

This, on the one hand, has a rewarding quality (see Van de Cruys \& Wagemans 2011) independent of the initial stimulus quality: we call this sudden Gestalt-forming event an "aesthetic aha!" or "Gestalt aha!" (Muth et al. 2013); actually such an aha is also paralleled by higher liking of the Gestalt- versus the non-
Gestalt-like display (Muth et al. 2013). This rewarding process points to an essential and very general mechanism of perception: to let people continuously seek meaning in visual displays. On the other hand, the rise in fluency facilitates faster and easier processing of the stimulus, resulting in a clearer representation of it, which allows for a more precise, amplified judgment in terms of FAM. Within this scope, the GANE model could help complement the cognitively described mechanisms of Gestalt recognition by a psychophysiological base. Even more generally, the core principle of perception can be interpreted by the GANE model as the intertwining process of selective amplification and inhibition to obtain the most clear interpretation of a given (e.g., visual) scene (Carbon 2014; Gregory 1970) and, thus, to enable the most appropriate action.

\section{Bodily arousal differentially impacts stimulus processing and memory: Norepinephrine in interoception}

\author{
doi:10.1017/S0140525X15001764, e205
}

\begin{abstract}
Hugo D. Critchley ${ }^{a, b, c}$ and Sarah N. Garfinkel ${ }^{a, b}$
${ }^{a}$ Sackler Centre for Consciousness Science, University of Sussex, Brighton,

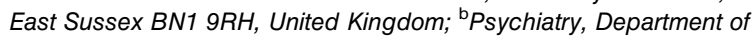
Neuroscience, Brighton and Sussex Medical School, Brighton, East Sussex BN1 9PX, United Kingdom; ' Sussex Partnership NHS Foundation Trust, Swandean Worthing, West Sussex BN13 3EP, United Kingdom.

h.critchley@bsms.ac.uk s.garfinkel@bsms.ac.uk

www.bsms.ac.uk/research/our-research/psychiatry

www.sussex.ac.uk/sackler/
\end{abstract}

Abstract: Bodily arousal modulates stimulus processing and memory, contributing to expression of emotional salience. The "glutamate amplifies noradrenergic effects" (GANE) model proposed by Mather and colleagues can be extended to account for the differential impact of interoceptive (notably cardiac afferent) signals on sensory processing. However, some emotion-specific effects, for example, for fear, may further depend on functional anatomical organisation of affect-related brain structures.

Mather and colleagues provide a compelling account of how stimulus processing is selectively prioritized through interaction of central noradrenaline (norepinephrine) and glutamate release. Their model explains discrepancies regarding the impact of central arousal on aspects of emotion, perception, and cognition. Thus, arousal sometimes enhances the processing of salient stimuli at the expense of neutral or contextual information, while, in other circumstances, it facilitates the processing of neutral stimuli and peripheral information. States of physiological arousal in the body evoked similar psychological effects, suggesting a common mechanism.

In presenting the GANE model, Mather and colleagues refer to studies of skydiving, threat response, processing of emotionally salient (alarming, exciting, or disturbing) stimuli, and loud noises. States of running and even unanaesthetized wakefulness in animal experiments are also considered. Arousal is proposed to be a common feature, operationally defined by noradrenaline release from the locus coeruleus. Such states of emotional and behavioural arousal are characterized by physiological changes in the periphery. Within the cardiovascular system, arousal is an embodied action-ready state: Heart rate and blood pressure increases are brought about by enhanced sympathetic drive, parasympathetic withdrawal, and baroreflex inhibition. Bodily arousal feeds back to influence perception, cognition, and emotion, and cardiac and arterial baroreceptors, which fire cyclically on each heartbeat, are a major source of these interoceptive influences.

Relevant to the GANE model, brainstem noradrenergic nuclei including locus coeruleus are sensitive to afferent interoceptive signals concerning bodily arousal. These nuclei support both 
descending control of autonomic function (A1 and A2 groups within medulla) and ascending control of alertness (e.g., A4 and A6 groups, including nucleus coeruleus). Correspondingly, they react to behavioural challenges by increasing sympathetic drive to the body and by increasing noradrenaline release in the brain via ascending projections from locus coeruleus to hypothalamus, thalamus, and forebrain (cortex and amygdala). Cardiovascular arousal is conveyed to the brainstem in a pulsatile manner by vagus nerve and glossopharyngeal afferents carrying the phasic discharge of baroreceptors that encode the timing and strength of individual heartbeats. The firing of locus coeruleus neurons is regulated by baroreceptor firing (Svensson 1987), resulting in cyclical inhibition of neural activity at late diastole (Elam et al. 1984; 1986; Morilak et al. 1986; Murase et al. 1994). Cardiac afferents modulate activity of nearby brainstem reticular nuclei (Lambertz \& Langhorst 1995) and even the amygdala, where the effect is also influenced by state of alertness (Lambertz et al. 1995). Vagus nerve stimulation enhances release of noradrenaline within the amygdala (Hassert et al. 2004).

Fine-grained signals concerning bodily arousal can thus influence perception and cognition via brain regions governing alertness and central arousal: Baroreceptor signals occurring with each heartbeat impact stimulus detection (Garfinkel et al. 2014; Park et al. 2014), memory (Garfinkel et al. 2013), and emotional responses (Garfinkel et al. 2014). Yet when it comes to processing emotional information, these physiological arousal signals evoke selective effects. Although cardiac systole inhibits the processing of pain stimuli (Gray et al. 2009) and attenuates the encoding into memory of words irrespective of valence (Garfinkel et al. 2013), the processing of fear stimuli is enhanced (Garfinkel et al. 2014).

The emotional attentional blink paradigm illustrates the prioritised processing of emotional stimuli. At the limit of perceptual awareness, emotional stimuli can overcome a perceptual block, the attentional blink effect, breaking through to awareness by capturing attention. This index of emotional salience is adrenergically mediated, being enhanced by administration of the noradrenergic reuptake inhibitor reboxetine and abolished by $\beta$-adrenoceptor blockade with propanolol (De Martino et al. 2008). This prioritized processing of emotional stimuli also depends on the functional integrity of the amygdala (Anderson \& Phelps 2001). The

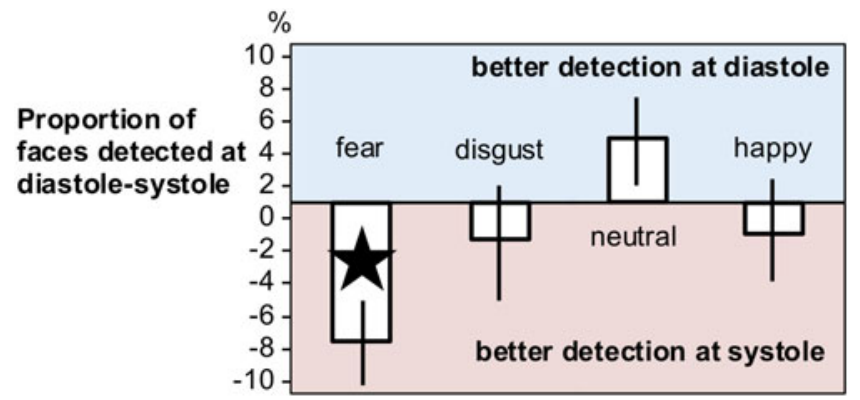

Figure 1 (Critchley \& Garfinkel). Cardiac modulation of emotional face detection. An attentional blink paradigm presents two target stimuli within a stream of masking distractors, pushing attentional resources to the limit for the perception of the second target stimulus presented during an "attentional blink" (about $300 \mathrm{~ms}$ after the first target). Detection of this second target is much better if the stimulus is emotional, reflecting intrinsic affective salience, and blocked by central $\beta$-adrenoceptor antagonists. The presentation of the second target (here faces) to coincide with cardiac systole (when arterial baroreceptors are active), compared with diastole (between these cardiac afferent signals), enhances the detection of fear stimuli, but has no effect on other emotion stimuli. (Adapted from Garfinkel et al. 2014). additional impact of afferent signals concerning cardiovascular arousal on early affective processing can be measured by timing the presentation of target stimuli to distinct phases (systole and diastole) of the cardiac cycle. Here the outstanding observation is a selective cardiac enhancement of fear processing, manifest in the emotional attentional blink task as better detection of fearful faces presented at systole, compared with diastole. This cardiac cycle effect is not seen for disgusted, happy, or neutral faces (although there is a trend for neutral faces to be better detected at diastole) (Fig. 1) (Garfinkel et al. 2014). Moreover, at systole, increased amygdala activity in response to fear compared with neutral stimuli predicts increased subjective rating of fear intensity and underscores the selective contribution of cardiac afferent signals to amygdala-mediated processing of salient stimuli (Garfinkel et al. 2014).

Thus, interoceptive signals concerning cardiovascular arousal can both increase (e.g., fear) and decrease (e.g., words, pain) stimulus processing. This is differentiated by the type of task or the emotion class of the stimulus. Although the GANE model explains much of the differential impact of cardiac afferent signals on sensory processing, it only partially accounts for emotion specificity and (task-related) behavioural demand that can further differentiate and guide the directionality of arousal effects. Encompassing physiological state within the concept of arousal reveals levels of interaction and a selective impact of the arousal signal itself. The glutamate component of the GANE model takes into account prioritisation of certain stimulus types, yet it underplays the degree to which this specificity must also depend on the differential anatomical organisation of critical brain structures supporting emotion-related response repertoires.

\section{Amplified selectivity in cognitive processing implements the neural gain model of norepinephrine function}

\section{doi:10.1017/S0140525X15001776, e206}

\author{
Eran Eldar, ${ }^{\mathrm{a}, \mathrm{b}}$ Jonathan D. Cohen, ${ }^{\mathrm{c}, \mathrm{d}}$ and Yael Niv ${ }^{\mathrm{c}, \mathrm{d}}$ \\ ${ }^{a}$ Wellcome Trust Centre for Neuroimaging, University College London, London \\ WC1N 3BG, United Kingdom; ${ }^{\mathrm{b}}$ Max Planck University College London Centre \\ for Computational Psychiatry and Ageing Research, London WC1B 5EH, \\ United Kingdom; ${ }^{\mathrm{C}}$ Princeton Neuroscience Institute, Princeton University, \\ Princeton, NJ 08544; ${ }^{\mathrm{d}}$ Psychology Department, Princeton University, \\ Princeton, NJ 08544. \\ e.eldar@ucl.ac.uk \\ sites.google.com/site/eldareran/ \\ jdc@ @rinceton.edu \\ www.csbmb.princeton.edu/ncc/ \\ yael@princeton.edu \\ www.princeton.edu/ nivlab/
}

Abstract: Previous work has suggested that an interaction between local selective (e.g., glutamatergic) excitation and global gain modulation (via norepinephrine) amplifies selectivity in information processing. Mather et al. extend this existing theory by suggesting that localized gain modulation may further mediate this effect - an interesting prospect that invites new theoretical and experimental work.

Mather and colleagues' article joins the growing body of work suggesting that norepinephrine, through its brainwide effect on neural gain, selectively enhances useful and salient neural representations (Aston-Jones \& Cohen 2005; Eldar et al. 2013; Usher et al. 1999). Building on an early computational model of catecholamine function (Servan-Schreiber et al. 1990), and later work directly addressing locus coeruleus function (Usher et al. 1999), Aston-Jones and Cohen (2005) proposed that one of the roles of the locus coeruleus-norepinephrine system is to enhance, through gain modulation, neural representations that are most useful for maximizing utility (adaptive gain theory). 
Critically, although norepinephrine is released globally throughout the brain, it was argued that its effects could be temporally and spatially specific. Temporally specific because norepinephrine can be phasically released in response to task-relevant stimuli and, thus, suitably timed to enhance representations that are most useful for task performance. Spatially specific because gain modulation inherently entails an interaction between norepinephrine and glutamate in which strong neural representations (i.e., those that are already receiving strong glutamatergic input, because of "bottom-up" sensory inputs and/or "top-down" context or control) are enhanced by norepinephrine, whereas weak neural representations are more inhibited (Eldar 2014; Eldar et al. 2013; see also Figure 5 in Mather et al.).

We conducted a series of behavioral and neuroimaging experiments to test this idea, that norepinephrine amplifies selectivity in information processing (Eldar 2014). Specifically, we investigated the relationship between selectivity and pupillometric indices of norepinephrine function in the domains of learning, perception, and memory. We first showed that indices of high norepinephrine function are associated with learning that is more selectively focused on stimulus features to which individuals are predisposed to attend (Eldar et al. 2013). We then showed that a similar effect is evident in the domain of perception. Specifically, we found that indices of high norepinephrine function are associated with perception of ambiguous characters that is more selectively focused either on the character's visual features or on its semantic context, depending on which source of information has stronger influence (we manipulated the source's strength using subliminal priming [Eldar 2014; Eldar et al., in press]). Notably, the latter finding suggests that norepinephrine will enhance bottom-up (e.g., visual features) or top-down (e.g., semantic) influences on perception, whichever is stronger. Finally, we also showed that a similar effect is evident in the domain of memory, where we found that indices of high norepinephrine function are associated with recognition memory that is more selective to the font in which a word appears, when attention is drawn to the font by the experimental task (Eldar 2014; Eldar et al. in press). These findings of increased selectivity in learning, perception, and memory were predicted by neural network models of norepinephrine function in which the effect of norepinephrine was modeled as a global increase in gain.

In addition to the behavioral predictions, our neural network models generated several neural predictions, which we tested using functional magnetic resonance imaging. First, increased gain entails that neural activity should be driven to maximal and minimal levels, and thus, the absolute deviation of activity levels from mean activity should increase with gain. Second, stronger responsivity to input signals should increase functional connectivity between neural units. Third, functional connectivity between neural units should become more selectively localized in clusters (i.e., less globally distributed), mirroring the behavioral selectivity that is associated with high gain. Indeed, pupillary indices of high norepinephrine function were associated with all three effects throughout the brain, as measured by brainwide blood oxygen level-dependent (BOLD) signals, further supporting the role of norepinephrine in global gain modulation in humans (Eldar et al. 2013).

The gain modulation model of norepinephrine function was originally inspired by findings that norepinephrine enhances singleneuron responses to both excitatory and inhibitory signals (e.g., Moises et al. 1979; Waterhouse \& Woodward 1980), which suggested that norepinephrine increases the contrast between strongly and weakly active neurons. However, subsequent single-neuron electrophysiology studies showed that norepinephrine may either enhance or suppress responsivity to excitatory input, depending on which receptor it activates (e.g., Devilbiss \& Waterhouse 2000). Mather and colleagues' proposal of local positive-feedback interaction between norepinephrine and glutamate reconciles this latter evidence with the neural gain model of norepinephrine function, because it suggests a mechanism through which the gainenhancing effect of norepinephrine would dominate specifically in strongly activated neurons, and thus, norepinephrine's overall effect would be to increase the contrast between weakly and strongly active neurons, as in the original model (shown in Fig. 5 in Mather et al.). In addition, the local changes in norepinephrine that Mather et al. propose may have additional effects that go beyond those of the interaction between local excitation and global gain modulation. For instance, local enhancement of gain may amplify selectivity even further. Indeed, such local changes have been suggested by early in vivo studies of the influence of sensory and thalamic inputs on cortical release of norepinephrine (e.g., Marrocco et al. 1987).

In sum, the neural gain model of norepinephrine function has been successful in predicting a range of norepinephrine's neural and behavioral effects, among which is amplified selectivity in perception and memory. Mather and colleagues' proposal of local glutamate-norepinephrine interaction further supports the neural gain model, suggesting that additional local interactions may enhance this effect. This suggestion invites further modeling to generate quantitative predictions and experimental work to test them.

\section{The role of arousal in predictive coding}

\section{doi:10.1017/S0140525X15001788, e207}

\section{Fernando Ferreira-Santos}

Laboratory of Neuropsychophysiology, Faculty of Psychology and Education Sciences, University of Porto, 4200-135 Porto, Portugal. frsantos@fpce.up.pt

\begin{abstract}
Within a predictive coding approach, the arousal/ norepinephrine effects described by the GANE (glutamate amplifies noradrenergic effects) model seem to modulate the precision attributed to prediction errors, favoring the selective updating of predictive models with larger prediction errors. However, to explain how arousal effects are triggered, it is likely that different kinds of prediction errors (including interoceptive/affective) need to be considered.
\end{abstract}

Classical models of information flow in the cerebral cortex consider that primary sensory regions detect the physical properties of the stimuli which are then combined into increasingly complex representations along the hierarchy of perceptual processing. As such, on the one hand perceptual processing is considered to be largely bottom-up, and top-down effects are expected to modulate the processing stream only. On the other hand, the predictive coding framework suggests that the cortical representation of objects is produced largely by top-down feedback to sensory cortices (i.e., predictions about what is being perceived originate in higher-level regions) (Clark 2013; Friston 2005; 2010). In this view, sensory information is not fed forward along the cortex, but, rather, what is communicated along the cortical hierarchy is only the difference between the predicted and actual inputs: the prediction errors. When such a mismatch occurs, the prediction errors are then used at the higher levels of the hierarchy to update the predictive model so as to eliminate prediction errors in the next round of comparisons (Clark 2013; Huang \& Rao 2011; Rao \& Ballard 1999). Predictions and prediction errors are thought to be instantiated by different neural units, and the balance between the two depends on precision cells that modulate their relative weights. Increased precision of the prediction errors means that the error signal will be strengthened by the precision units and lead to a stronger updating of the predictive model, whereas decreased precision suppresses the prediction errors and, thus, maintains the current model (Barrett \& Simmons 2015). With this brief introduction in mind, I now turn to how the GANE (glutamate amplifies noradrenergic effects) model may be integrated within a predictive coding approach - a possibility that is acknowledged by Mather et al.

The activity of norepinephrine (NE) neurons has been in the focus of researchers interested in the neural coding of prediction 
errors (Dayan \& Yu 2006). NE cells respond phasically to unexpected stimuli across sensory modalities and cease to respond after a few repetitions of the stimulus, a pattern of activity that is consistent with what would be expected from units coding prediction errors (Schultz \& Dickinson 2000). As detailed by the GANE model, however, the overall effect of NE seems to be more akin to a modulation of the precision weights of prediction errors. Thus, in predictive coding terminology, NE amplifies the stronger feedforward glutamatergic error signals while suppressing weaker prediction errors, leading to a stronger updating of only the most unexpected inputs. Indeed, this is a sensible explanation: strong prediction errors signal highly unexpected sensory input and, thus, elicit orienting responses and concomitant central NE release to boost signal-to-noise ratio and favor the updating of the most relevant predictions.

The salience or priority of stimuli that seem to trigger NE effects, however, is not fully dependent on sensory mismatch. It is true that phasic NE responses occur to intense unexpected sensory inputs (Petersen \& Posner 2012), but also to stimuli that are not physically extreme, namely, stimuli that carry emotional or task-related significance (Schultz \& Dickinson 2000). Indeed, the affective/motivational aspect of arousal is something that has not been the focus of the more classic formulations of predictive coding approaches. However, recent models of affective predictive coding extend the predictive coding framework, originally developed to account for perception of external objects, to include interoception, that is, the cortical representation of internal states that constitute the basis of emotional experience (Barrett \& Simmons 2015; Seth 2013). Also, affective predictive coding models do not consider interoceptive inferences as independent from exteroceptive processing, but rather consider that affective predictions and affective prediction errors are basic components of "regular" perception (Barrett \& Bar 2009). This means that the perception of an object involves predictions not only about its physical features (e.g., shape, color), but also about its affective properties (e.g., very pleasant, neutral, scary), and that the prediction errors that are elicited may concern sensory and affective mismatches.

One hypothesis consistent with this view is that engagement of NE neurons in the locus coeruleus may depend on a threshold of the net sum of prediction errors for a given input. This would mean that arousal effects may occur following sensory, affective, or task-related mismatch (depending on whether the stimulus is, respectively, inconsistent with perceptual, interoceptive, or goalrelated predictions) or a combination of these. If this combination of prediction errors reaches a given threshold, then a phasic NE response is elicited to facilitate the selective updating of predictions in the prioritized manner that Mather and colleagues elegantly describe. Indeed, it has been reported that emotionally deviant stimuli evoke larger cortical prediction errors than neutral deviants (Vogel et al. 2015a), but the precise role of $\mathrm{NE}$ in this effect remains an issue for future investigation.

\section{ACKNOWLEDGMENT}

Fernando Ferreira-Santos is supported by a grant from the BIAL Foundation (242/14).

\section{Are there "local hotspots?" When concepts of cognitive psychology do not fit with physiological results}

\author{
doi:10.1017/S0140525X1500179X, e208
}

\section{Quentin Gaucher and Jean-Marc Edeline}

Paris-Saclay Institute of Neurosciences (Neuro-PSI), University Paris-Sud, CNRS, and Paris-Saclay University, 91405 Orsay Cedex, France. jean-marc.edeline@u-psud.fr gaucher.quentin@gmail.com http://neuro-psi.cnrs.fr/spip.php?article135
Abstract: Mather and colleagues' arguments require rethinking at the mechanistic level. The arguments on the physiological effects of norepinephrine at the cortical level are inconsistent with large parts of the literature. There is no evidence that norepinephrine induces local "hotspots": Norepinephrine mainly decreases evoked responses; facilitating effects are rare and not localized. More generally, the idea that perception benefits from "local hotspots" is hardly compatible with the fact that neural representations involve largely distributed activation of cortical and subcortical networks.

Mather and colleagues propose that phasic activation of LC neurons biases perception and memory. They suggest that elevated levels of glutamate at the site of prioritized representations increase local norepinephrine (NE) release, creating "NE hotspots." At these spots, enhancement of glutamate and release of NE mutually enhance and amplify the activation of prioritized representations. This excitatory effect contrasts with the widespread suppression by $\mathrm{NE}$ of weaker representations via lateral and auto-inhibitory processes.

Mather et al. provide a schematic representation at the cellular level (Fig. 6), but is it supported by physiological data obtained in sensory cortices? Although the locus coeruleus (LC) neurons project widely to many cortical areas, recent data indicate that some neurons project more to one area (the prefrontal or motor cortex) than others (Chandler et al. 2013; 2014). Within an area, $\mathrm{NE}$ is released in the extracellular space from $\mathrm{NE}$ varicosities and reaches the entire cortical network. When sensory stimuli are processed by cortical neurons, glutamate is released by the thalamocortical terminals. How does NE affect cortical processing? Iontophoretic application of NE performed in the somatosensory, visual, and auditory cortices revealed that, in most of the cases, NE depressed evoked responses (e.g., Kolta et al. 1987; Manunta \& Edeline 1997; 1998; Videen et al. 1984), an effect replicated in awake animals (Bassant et al. 1990; Foote et al. 1975; Manunta \& Edeline 1999). Moreover, in awake rats, tonic activation of LC neurons by continuous low-frequency stimulation $(1 \mathrm{~Hz})$ triggered similar effects: decreased evoked responses in $63 \%$ of cells in the rat somatosensory cortex (Devilbiss \& Waterhouse 2004). It has been argued that these inhibitory effects were a consequence of the very high concentrations of NE in the vicinity of the cell (Waterhouse et al. 1998a), but this seems unlikely given that pronounced depression of evoked responses was also observed with very low ejection currents (Ego-Stengel et al. 2002; Manunta \& Edeline 1997; reviewed in Edeline 2012). If the hotspot theory were the main mechanism at play, then exogenous application of NA would more likely have increased evoked activity.

But what are the consequences for the neurons' functional properties? In the auditory cortex, the suppressive effect of NE promotes an increase in frequency selectivity in both anesthetized and unanesthetized animals (Edeline 1995; Manunta \& Edeline 1997; 1999). In the visual cortex, application of NE improved the velocity and direction selectivity of cells, without modifying orientation selectivity (Ego-Stengel et al. 2002; McLean \& Waterhouse 1994). These results clearly point out that the effects of NE (and other neuromodulators) can differ depending on the stimulus dimension. For example, a dimension that depends on thalamocortical afferences (such as frequency tuning in the auditory cortex or size of the receptive field in the visual cortex) could be more affected than a dimension that relies more on corticocortical afferences (such as frequency modulation tuning in the auditory cortex or velocity tuning in the visual cortex). Yet, glutamate is released in all cases, indicating that the glutamate-norepinephrine interaction is not as straightforward as described by the authors.

One may ask if it is possible for "NE hotspots" to emerge when $\mathrm{NE}$ is repeatedly associated with glutamate release at particular synapses? When a stimulus that activates a specific set of synapses is paired with phasic LC stimulation, a predominant decrease in neuronal activity is initially reported in several cortical areas (Olpe et al. 1980; Sato et al. 1989). However, in the somatosensory cortex, both the excitatory and inhibitory components of evoked responses are facilitated when phasic stimulation of the LC is 
delivered before tactile stimuli (Snow et al. 1999; Waterhouse et al. 1998b). Rather, in several sensory modalities, LC stimulation affects the temporal organization of evoked responses (Bouret \& Sara 2002), by shortening evoked responses and reducing the first spike latency and its variance (Lecas 2001; 2004). In the auditory cortex, facilitation of evoked responses is the dominant effect when stimulation of the LC is delivered before a particular sound frequency (Edeline et al. 2011; Martins \& Froemke 2015), which contrasts with the dominant depressive effect obtained when phasic pulses (1 s) of NE are iontophoretically delivered in the vicinity of the recorded cells (Manunta \& Edeline 2004). In fact, via its projections onto other brain nuclei, LC stimulation activates other neuromodulatory systems such as the cholinergic (Berridge and Foote 1991; Berridge et al. 1993) and serotoninergic (Kim et al. 2004) systems. The difference between the effects obtained with iontophoretic application of $\mathrm{NE}$ and LC stimulation indicates that other neuromodulators likely contribute to the effects observed at the cortical level.

Mather et al. also suggested that local "NE hotspots" bias perception. But is there any evidence that NE facilitates the discrimination performance of cortical neurons? When the responses of cortical neurons are tested with a set of conspecific and heterospecific vocalizations having the same spectral content (thereby activating the same sets of synapses), NE application induces either an increase or a decrease in the response, and, on average, discrimination of overall performance of cortical neurons is unaffected. However, a small population of neurons displaying the largest increase in responses exhibit enhanced discrimination (quantified by the mutual information) between communication sounds (Gaucher \& Edeline 2015). This population of neurons corresponds to recordings showing smaller receptive fields; they are not located in a particular layer and are distributed across the whole cortical map. As for any natural stimulus, representations of these vocalizations are distributed among largely overlapping cortical and subcortical networks. Therefore, having local "NE hotspots" in such networks will not help perception of such stimuli. Subtle, and transient, synchronizations between distributed populations of neurons are potentially more relevant for discriminating natural stimuli.

To conclude, based on physiological results from sensory cortices, it is clear that the dominant effect of NE is widespread suppression of excitatory inputs when responses are tested both with artificial and with natural stimuli. NE promotes facilitated responses for only a small proportion of cells; this effect is more common with LC activation, probably because of activation of other neuromodulatory systems. Only a small fraction of these facilitated responses displayed enhanced discriminative performance. Therefore, the local "NE hotspot" theory receives little support from current physiological results. Although we cannot dismiss that the "hotspot theory" might account for certain specific forms of perceptual alterations, we believe this theory is inappropriate to account for facilitations of perceptive abilities. It remains to be demonstrated that local interactions between NE and glutamate promote enhanced perceptive abilities, particularly because of the inherent distributed representation of any natural stimuli.

\section{Contemplating the GANE model using an extreme case paradigm}

\section{doi:10.1017/S0140525X15001806, e209}

\section{Ronny Geva}

Department of Psychology, Gonda Brain Research Center, Bar-llan University, Ramat Gan 5290002, Israel.

ronny.geva@biu.ac.il

Abstract: Early experiences play a crucial role in programming brain function, affecting selective attention, learning, and memory. Infancy literature suggests an extension of the GANE (glutamate amplifies noradrenergic effects) model to conditions with minimal priority-map inputs, yet suggests qualifications by noting that its efficacy is increased when tonic levels of arousal are maintained in an optimal range, in manners that are age and exposure dependent.

Mather and colleagues' intriguing GANE (glutamate amplifies noradrenergic effects) model underscores an important process, through which GANE changes influence the selection process to favor high- over low-priority representations.

The extended literature covered in the article concentrates mostly on experimental research with typically developing young adults, whose performance relies on an established neural network, set with implicit "know-hows" and an explicit knowledge base, which shape and set local hotspots, to be activated proactively in the prospect of newly arriving inputs (Bouret \& Richmond 2015). One way to test this model may be an extreme case paradigm in which top-down priorities are negligible, and the roles of global brain activation are augmented, as is the case of the newborn.

Research on infancy, early development of attention, and arousal, in typical and clinical samples opens the discussion of the generalizability of the GANE model because young infants perceive stimuli with no preset priorities and with little previous knowledge. As such, infancy offers an interesting test case for the GANE model.

Early-life experiences play a crucial role in programming brain function, particularly with respect to selective attention, learning, and memory (Geva et al. 2006). Newborns are busy perceiving and memorizing the environment at rates that are not surpassed thereafter, equipped with an impressionable template that does not allow yet for exerting deliberate priority operations. How might GANE function at infancy?

Models with neonates highlight four interdependent notions that may qualify the limits of the proposed model, with respect to development, exposure, global activity, and resilience to variance.

Dependence on development. Neonates and adults differ markedly in their ability to learn selectively (Kuhl et al. 1992). These differences were suggested to be related, in part, to developmental differences in arousal response to sensory stimuli (Kuhl 2007) as a function of differential locus coeruleus-norepinephrine (LCNE) activity (Moriceau \& Sullivan 2004; Nakamura \& Sakaguchi 1990). Differences are such that compared with the LC of the infant, the adult LC gradually becomes less likely to respond to non-noxious stimuli (Kimura \& Nakamura 1985; Nakamura et al. 1987; Selden et al. 1990), habituates earlier in response to repeated (or even single) stimulation (Vankov et al. 1995), and produces shortened LC responses in response to sensory stimulation (Nakamura \& Sakaguchi 1990). All of these differences suggest a potential role for development in the proposed model (Moriceau \& Sullivan 2004).

Dependence on experience. Exposure at sensitive periods seems to play a significant role in the development of the LCNE system (Nakamura et al. 1987; Rangel \& Leon 1995). Also, experience early in development has been found to affect PFC responsivity to LC-NE. For example, neonatal experience involving maternal contact reward was reported to affect the noradrenergic system of the rat prefrontal cortex (Kalpachidou et al. 2015). The experience was related to hypomethylation of the $\beta_{1}$-adrenergic receptor gene promoter and consequently enhanced expression of its mRNA in the prefrontal cortex, resulting in better discrimination and improved learning in the young pups (Kalpachidou et al. 2015).

In addition, selective recognition of maternal odors has been found to be accompanied by increased release of glutamate and GABA from the dendrodendritic synapses and an increased efficacy of glutamate-evoked GABA release (Kendrick et al. 1992), and early-life stress related to maternal separation has been reported to alter glutamate and GABA transmission and, in particular, to alter $\mathrm{GABA}_{\mathrm{A}}$ receptor expression (Sterley et al. 2013). 
The integration of these findings points to the possible role of early-life exposure in the GANE model.

Dependence on tonic levels of activity. The LC is thought to play a central role in regulating arousal states in addition to its role in attention and memory (Howells et al. 2010; Rajkowski et al. 1994). Initial leads from human infancy research point to the notion that in the case of the newborn, arousal homeostasis possibly plays a significant role in attention and in recognition (Geva et al. 1999), with brainstem pathways playing a central role in gating arousal self-regulation (Geva \& Feldman 2008). Feedingdependent arousal differences were found to affect newborn preferences for cognitively demanding stimuli (Geva et al. 1999; 2013), the interaction is such that more aroused neonates tend to orient toward less intense familiar stimuli; yet when less aroused, newborns prefer more intense stimuli (Gardner \& Karmel 1983; 1984) and orient toward novel stimuli as compared with familiar ones in visual recognition memory tasks (Geva et al. 1999).

Sleep-wake arousal states also seem to play a similar role. Recent work with intracellular recordings has shown an interaction of LC activity in monkeys as a function of fatigue, an effect attributed to the LC possibly providing the impetus to act when the predicted outcome value is low (Bouret \& Richmond 2015). Indeed, arousal states were found to affect attention in young human infants. Neonatal sleep fragmentation was reported to be associated with infants' focused attention to specific stimuli early in development (Geva et al. 2013). Compared with good sleepers, infants who were poor sleepers as neonates had difficulties focusing on target stimuli in the presence of complex distracters, but managed focusing in the presence of simpler distracters. Integration of the findings on arousal state effects on attention and memory emphasizes the need to consider tonic arousal changes in the GANE model.

Finally, the validity of the model may gain from testing of its limits in neuropsychiatric disorders, such as attention-deficit/ hyperactivity disorder (Sterley et al. 2013), which involve poor adaptation to change (Sara 2009). Such an exploration may suggest the notion that GANE efficacy is increased when tonic levels of arousal are maintained in an optimal range.

Together, these data suggest an extension of the GANE model to infancy; however, integration of the above findings with the framework presented suggests a qualification to the GANE model, by noting that its efficacy is increased when tonic levels of arousal are maintained in an optimal range, in manners that are age and exposure dependent.

\section{Dentate gyrus and hilar region revisited}

doi:10.1017/S0140525X15001818, e210

\section{Conor Houghton \\ Department of Computer Science, University of Bristol, Bristol BS8 1UB, United Kingdom. \\ conor.houghton@bristol.ac.uk \\ http://www.cs.bris.ac.uk/home/cscjh/}

Abstract: It is suggested that the dentate gyrus and hilar region in the hippocampus perform memory selection and that the selectivity of the gating of memory by this circuit is modulated by the norepinephrineglutamate loop described by Mather et al.

Mather et al. propose that arousal modulates attention through a norepinephrine-glutamate feedback loop in local circuits. Here, I suggest a specific circuit where this mechanism may be in operation: the granule cell-mossy cell loop in the hippocampus.

It is commonly proposed that the CA 3 region of hippocampus forms an auto-associative memory store for short- and mediumterm memories (Gardner-Medwin 1976; Hopfield 1982; Levy \& Steward 1979; Marr 1971; McNaughton \& Morris 1987; Rolls
1989; Treves \& Rolls 1992). Here, pictures, memories, in the form of patterns of activity in the entorhinal cortex, feed forward along the perforant pathway to CA3, activating a sparse subset of the CA3 pyramidal cells. Plasticity in the synapses of the recurrent network in CA3 and in the perforant pathway synapses onto CA3 neurons fixes the memory so that it can be recalled: If a part of the same pattern of activity occurs in entorhinal cortex, the corresponding part pattern is activated in CA3 and it is then completed by auto-associative dynamics.

Pattern collision, where two similar memories are confused during pattern completion, is a problem in auto-associative networks, particularly if they are required to rapidly store memories with only a small number of presentations. It is likely that the hippocampus has a mechanism to avoid or reduce pattern collision: the hippocampus stores rapidly acquired memories, and it is important that similar but distinct memories can be distinguished during recall.

It has been proposed that the role of the dentate gyrus is to separate patterns and thereby reduce collisions (Gilbert et al. 2001; Leutgeb et al. 2007; McHugh et al. 2007; O’Reilly \& McClelland 1994; Treves \& Rolls 1992). In addition to CA3 neurons, the perforant pathway connects to the granule layer in dentate gyrus. The granule layer of dentate gyrus is, in turn, connected to CA3 along the mossy fibers. This means that the entorhinal cortex is connected to CA3 directly, along the perforant pathway, and indirectly, via dentate gyrus. In the specific version of dentate gyrus pattern separation proposed by O'Reilly and McClelland (1994), there is local k-winner-take all dynamics between cells in dentate gyrus, and the consequence of this is that only a random subset of the cells receiving input from entorhinal cortex become active. This activity is fed forward along the mossy fibers to CA3 and, in turn, excites a random subset of those cells in CA3 that receive input from entorhinal cortex. This randomization separates the patterns that are then learned in the CA3 auto-associative network.

There is experimental evidence (McHugh et al. 2007) that the dentate gyrus is important for pattern separation and that the adult neurogenesis of dentate gyrus granule cells, which may support the randomization, is linked to pattern separation (Altman 1963; Bayer et al. 1982; Clelland et al. 2009; Sahay et al. 2011). However, it seems unlikely that pattern separation is the only role of the dentate gyrus; for a start, pattern separation on its own seems a modest role for such a substantial brain region. Beyond this, pattern separation does not explain either the hilar region or the role of norepinephrine in the dentate gyrus.

The hilar region lies between dentate gyrus and CA3. As the mossy fibers run through the hilar region they form en passant connections with the mossy cells (Amaral 1978; Scharfman \& Myers 2013). These are large excitatory cells whose proximal dendrites are covered in mossy-looking spines. The mossy cells, in turn, have a substantial backprojection that extends along the longitudinal axis of the dentate gyrus (Amaral \& Witter 1989; Amaral et al. 2007) and connects to both granule cells and inhibitory interneurons (Scharfman 1994; 1995).

This two-layer structure seems more elaborate than a simple randomizing k-winner-takes-all network would require; random subselection from a pattern could be achieved by local excitatory-inhibitory dynamics within the dentate gyrus itself. However, the two-layer structure would make sense if the role of the dentate gyrus encompassed memory selection as well as pattern separation. As pointed out by Koch et al. (Koch \& Ullman 1984; 1987; Olshausen et al. 1993), a single layer winner-takes-all network in which competition occurs across the whole network requires considerable interneuronal connectivity. This issue is resolved by having more than one layer; in the first layer, competition is restricted to subregions, and a champion emerges from each subregion to compete in the next layer where the competition between subregions occurs. In short, I suggest here that, in addition to separating patterns, the winnertake-all dynamics in the dentate gyrus also compares the salience 
of different aspects of its input and that this selection gates and refines the storage of memories in CA3. The role of the hilar region is to facilitate this comparison.

The locus coeruleus projects to the dentate gyrus, which contains $\beta$-adrenergic receptors (Berridge \& Waterhouse 2003; Harley 2007). Norepinephrine release in response to novelty during exploration enhances excitability in the dentate gyrus (Kitchigina et al. 1997); in fact, the activity of both interneurons (Nitz \& McNaughton 2004) and excitatory neurons (Dahl \& Winson 1985; Neuman \& Harley 1983) in dentate gyrus show norepinephrine-promoted increase in response to novelty. Furthermore, it has been reported that in hippocampus, glutamate causes enhanced norepinephrine release (Pittaluga \& Raiteri 1990; Raiteri et al. 1992), an effect that is most marked in the dentate gyrus (Andrés et al. 1993). Conversely, norepinephrine in dentate gyrus, but not in other hippocampal regions, potentiates the release of glutamate (Lynch \& Bliss 1986). The role of norepinephrine in dentate gyrus seems somewhat mysterious if the role of the dentate gyrus is restricted to pattern separation. However, if, as proposed here, the dentate gyrus also performs memory selection, then the norepinephrine-glutamate mechanism for modulating memory selectivity described by Mather et al. becomes the missing clue that could explain the role of norepinephrine in dentate gyrus.

\section{GANEing on emotion and emotion regulation}

\section{doi:10.1017/S0140525X1500182X, e211}

\section{Thomas D. Hull}

Department of Counseling and Clinical Psychology, Columbia University, New York, NY 10027.

tdh2120@columbia.edu

\begin{abstract}
The function of emotion and its underlying neural mechanisms are often left underspecified. I extend the GANE (glutamate amplifies noradrenergic effects) model by examining its success in accounting for findings in emotion regulation. I also identify points of alignment with construction models of emotion and with the hypothesis that emotion states function to push neural activity toward rapid and efficient action.
\end{abstract}

What is emotion? Why did it evolve and what is its purpose? Several models of the origin and function of emotion have been put forward (see Gross \& Barrett 2011 for review). For the sake of brevity, I identify two broad and encompassing approaches here. One pursues questions related to why and how emotions evolved (Ekman 1993; Tooby \& Cosmides 1990), referred to as the entity view. The other focuses on why emotion evolved (Lindquist et al. 2012; Simon 1967), and that one I will call the process view. At first glance it is a subtle difference, but it is a difference that matters greatly for how we understand emotion, its underlying mechanisms, and its adaptive functions.

From the entity view, individual emotions served specific functions in the past that were important for survival and so were preserved. Each emotion is structured like an organ that has feature detectors for identifying relevant stimuli that then trigger a coordinated set of action tendencies that enhance survival (Panksepp 2007). The challenge for modern humans is to regulate these inherited responses to conform to the much changed present-day environment. The process view, on the other hand, allows for a nearly unlimited variety of emotional states and responses. Principles of neural computation are often an important part of this account (Lindquist et al. 2012), and can be augmented by stipulating that the function of emotion is to enable neurologic systems to minimize exploration over the current problem space to more rapidly bring about efficient action (cf. Donoso et al. 2014; Simon 1967), something we refer to as computational expediency.

The GANE model is an intriguing mechanism, supportive and suggestive of the process view of emotion insofar as it binds any variety of prioritized cortical representations to arousal and core affective states, rather than assuming that an individual category of emotion (sadness or fear, for example) produces stereotyped cognitive and behavioral effects. Thus, GANE suggests that there is a great deal of flexibility in the formation of emotion states and that no special neural substrates or modules of particular emotions are needed to account for the adaptive, and sometimes maladaptive, nature of emotional memory and responding. This agrees with the increasingly influential models of emotion construction; however, a full exposition is beyond the scope of this commentary.

I instead focus on the principle of computational expediency within the framework of GANE by drawing on findings from emotion regulation. An inability to modulate arousal may lead to difficulties in adapting to present circumstances, not because emotional states are geared toward environments from our phylogenetic past, but because alternative and more adaptive forms of responding may not reach priority in the cortex. If one's affective learning history prioritizes maladaptive cortical representations because of social modeling, maltreatment, or potentially traumatic low-probability events, GANE suggests that unless there is a dampening of arousal, or assistance in pushing subthreshold representations into greater excitation, or both, it will be difficult to alter behavioral responses. Less clinically, because the neural categorization of stimuli and situations is probabilistic (Donoso et al. 2014), cortical activity that represents situations will inevitably err from time to time. Giving oneself space to explore alternatives through arousal regulation efforts is likely to help individuals recover from misattributions and behave more adaptively. Reducing arousal through emotion regulation would provide an opportunity for neural activity representing alternatives to reach priority and, thus, have an impact on action and memory.

For these reasons, emotion regulation is a ubiquitous human activity (Gross 2015), and recent research indicates that it can take on a variety of forms depending on the situation and one's goals. Humans often increase emotional states that they believe will enhance their performance (Tamir et al. 2015). For example, when preparing for an upcoming negotiation, individuals will increase negative emotional states (such as anger) to increase the likelihood of obtaining their goal. We could speculate that the arousal and prioritized representations we label as anger in this instance provide a singularity of focus and purpose unencumbered by the deliberation of alternative states as suggested by GANE mechanisms. Relatedly, the strategy of distraction is more effective for high-intensity stimuli, whereas altering one's interpretations (a strategy called reappraisal) is more effective for low-intensity stimuli (Sheppes et al. 2014). We suggest that distraction during high-arousal events helps to reduce the tendency toward computational expediency of prioritized representations, to allow for further exploration of the dangers, demands, and opportunities of the situation. When arousal is low, reappraisal is more successful at prioritizing new representations so that new memories can be formed that change how one would respond to the stimulus in the future (Denny et al. 2015). Importantly, the success of reappraisal is severely impaired if stress and arousal are high (Raio et al. 2013), perhaps because cortical activity representing alternative interpretations is unable to reach the high-arousal threshold and achieve priority.

A final intriguing case is the impact that cognitive load has on reducing hedonic arousal and temptation (Van Dillen et al. 2013). As Mather et al. note, many models of cognition and emotion assume that emotionally evocative stimuli always take priority over attention. However, findings that cognitive load interferes with the introduction of new emotion states further support the GANE model by demonstrating how current prioritized representations are maintained by arousal to the exclusion of alternative representations, even those that would otherwise be emotional given one's affective learning history.

It is not my intention to argue that all aspects of emotion, emotion regulation, or psychopathology can be accounted for by the GANE model. Numerous other neurotransmitters and 
neuromodulators will also play decisive roles. However, the GANE model offers a neural mechanism that helps to unify cognition and emotion while drawing attention to neurocomputational effects that align with previous theorizing on the function of emotion in ways that are suggestive of future research on the mechanistic bases of emotion regulation.

\section{Once more with feeling: On the explanatory limits of the GANE model and the missing role of subjective experience}

\author{
doi:10.1017/S0140525X15001831, e212
}

\author{
Jeffrey R. Huntsinger ${ }^{a}$ and Justin Storbeck ${ }^{b}$ \\ ${ }^{a}$ Department of Psychology, Loyola University Chicago, Chicago, IL 60660; \\ ${ }^{\mathrm{b}}$ Department of Psychology, Queens College, and the Graduate Center, City \\ University of New York (CUNY), Flushing, NY 11367. \\ jhuntsinger@luc.edu \\ http://jeffreyhuntsinger.weebly.com/index.html \\ justin.storbeck@qc.cuny.edu \\ http://www.queensaffectiveneuroscience.com/
}

Abstract: We applaud Mather and colleagues' model, which emphasizes the neurobiological pathways by which affective arousal tunes attention and memory. This commentary offers a friendly discussion of several potential limitations of the theory. We suggest the model is strong when predicting task-driven demands but is limited when predicting the impact of individual biases, interpretations, and experiential feelings.

Mather et al. introduce an impressively broad neurobiological model of the role of affective arousal in directing attention and memory. Rather than discuss the many strengths of the GANE (glutamate amplifies noradrenergic effects) model, our commentary offers a friendly discussion of some limitations of the model and the missing role of subjective experience.

One concern is the predictive utility of the model. The model can account for a variety of effects, but it fails at making clear a priori predictions for attention and memory effects. One reason it lacks predictive utility is its reliance on salience. The use of salience falls victim to a circular argument, because salience often depends on confirmation from the results (self-dependent justifications) (Hahn 2011). Such circularity hampers theory prediction because salience concedes vagueness as it becomes defined post hoc or through task demands. For instance, imagine that participants were asked to attend to a central fear face and ignore surrounding faces. One study used neutral faces as distractors and found better attention/memory for fear faces, but another study used angry faces as distractors and found better attention/ memory for angry faces. The vagueness of saliency allows for both studies to support the model (saliency determined by task demands and stimuli, respectively); yet, a naïve researcher would fail to make these distinct predictions with the GANE model. Thus, the model can account for various effects, but fails to make clear, deductive predictions (such circularity plagued the depth of the processing approach) (Craik \& Lockhart 1972).

The model also does not address predictions based on individual differences. If we compared memory for task-dependent salient stimuli (snakes) in the face of distractors (spiders), at a group level, people may show better memory for snakes than spiders. However, would this be true for each individual? Probably not. Spider-phobics may remember the spider rather than the snake. Therefore, can the model accurately predict when task demands or individual biases will have a greater impact on attention/memory? Moreover, can this model be extended to predict attentional/memory biases linked to various mental health disorders (depression, anxiety, attention deficit/hyperactivity disorder)?

Attention and memory are treated objectively in the model, but both are often susceptible to subjective experiences. False memory studies demonstrate such vulnerabilities (Loftus 1975; Roediger \& McDermott 1995). For example, people learning a list of words (bed, pillow, wake, ...) that are highly related to a single, non-presented word (sleep) often falsely recall that nonpresented word. Payne and colleagues (2002) stressed participants prior to a false memory task and found that false memories and cortisol levels were positively correlated. Does the current model make such a prediction? Based on the review, one would predict a decrease in false memories because norepinephrine enhances the signal (presented words) and reduces the noise (non-presented words). But such a prediction would not be supported. Therefore, does the model reduce noise only for perceptually based stimuli? Alternatively, how does the model explain subjective experiences (i.e., associative and conceptual processes) gaining saliency? Where would norepinephrine hotspots arise within the brain when false memory effects are present?

Finally, the GANE model is a neurobiological account of affective arousal. Not surprisingly, then, the model focuses exclusively on this component of arousal. We suggest that in doing so, the model fails to consider the important role played by the subjective-experiential component of arousal and associated implicit attributions.

According to an affect-as-information approach, affective arousal serves as experiential information by signaling importance or urgency (Clore \& Huntsinger 2007; Storbeck \& Clore 2008). Any source of affective arousal can modulate judgment, attention, and memory as long as it is implicitly attributed to or associated with an object of judgment, current attentional focus, or memory.

Research by Cantor et al. (1975) indicates that cues of affective arousal are easily transferred or misattributed from one source to another. In this research, participants experienced high arousal via exercise and were then shown an erotic film. Highly aroused participants rated the film more positively. However, this effect vanished when participants' attention was called to the true source of their arousal. Although both groups experienced the same pattern of neurophysiological change from arousal, arousal influenced judgments only for participants who misattributed the source of their arousal to the film.

Dutton and Aron's (1974) classic bridge study further illustrates the role of implicit attribution in the influence of affective arousal on judgment. In that study, male passersby were approached by an attractive woman interviewer who administered a questionnaire on either an unsteady, high-arousal suspension bridge or a stable, low-arousal bridge. Once the questionnaire was completed, the interviewer offered to explain the experiment in more detail at a later time. She then handed the men a piece of paper with her phone number written on it and encouraged them to call if they had any questions. The investigators found that the men approached by the attractive interviewer on the unsteady bridge were more likely to call her than those approached on the stable bridge. The men on the unsteady bridge misattributed their feelings of arousal caused by the bridge as a reaction to the interviewer, thus intensifying feelings of attraction to her.

The bridge study raises an interesting question for the GANE model. The model argues that arousal should enhance memory for mental representations active at the moment arousal is induced. In the bridge study, arousal begins on the bridge, but ultimately the attractive woman gains attention. According to the GANE model, will arousal enhance memory for the scary bridge or the attractive woman?

In summary, research on misattribution of arousal suggests that neurobiological change elicited by the experience of affective arousal is not necessarily the driver of its effects on many outcomes. Rather, the experiential information on importance and urgency carried by affective arousal and the implicit attribution of arousal are crucial.

We want to end by emphasizing that the criticisms offered here are in the spirit of improving the GANE model. Indeed, there is much to like about the model and we agree more than disagree with much of it. 


\section{Interactions of noradrenaline and cortisol and the induction of indelible memories}

\section{doi:10.1017/S0140525X15001843, e213}

\author{
René Hurlemann,, Wolfgang Maier, ${ }^{a, c}$ and Dirk Scheele ${ }^{a, b}$ \\ ${ }^{a}$ Department of Psychiatry, University of Bonn, 53105 Bonn, Germany; \\ ${ }^{\mathrm{b} D i v i s i o n}$ of Medical Psychology, University of Bonn, 53105 Bonn, Germany; \\ ${ }^{\circ}$ German Center for Neurodegenerative Diseases (DZNE), 53175 Bonn, \\ Germany. \\ renehurlemann@icloud.com \\ wolfgang.maier@ukb.uni-bonn.de \\ dirk-scheele@gmx.de \\ http://renehurlemann.squarespace.com/welcome/ \\ http://psychiatrie.uni-bonn.de/
}

Abstract: The glutamate amplifies noradrenergic effects (GANE) model emphasizes the role of focal glutamate-noradrenaline interactions in creating functional hotspots for prioritized processing of salient stimuli. Here, we briefly outline current evidence that synergistic action of noradrenaline and cortisol enables emotional stimuli to gain privileged access to amygdala-hippocampus circuits, eventually resulting in the formation of indelible memories and posttraumatic stress disorder (PTSD).

In their superb glutamate amplifies noradrenergic effects (GANE) model, Mather and colleagues convincingly argue that under conditions of arousal-induced phasic activity of the locus coeruleus (LC), locally elevated glutamate (GLU) levels amplify noradrenergic (norepinephrine [NE]) release from the LC, thus creating functional hotspots of prioritized processing that bias perception and memory. Although the GANE model focuses on stimulus salience coding through rapid GLU and NE signaling and their focal interactions, it should be emphasized that endocrine signals, including the adrenal stress hormone cortisol (CORT), brain concentrations of which peak within minutes as a result of hypothalamus-pituitary-adrenal (HPA) axis activation (de Kloet et al. 2005), also intimately interact with NE to code perceptual and mnemonic priority, especially under conditions of emotional arousal.

In functional magnetic resonance imaging (fMRI) experiments, emotional arousal is frequently operationalized by exposing subjects to facial displays of emotion, which evoke responses in specific functional subdivisions of the amygdala (Goossens et al. 2009; Hurlemann et al. 2008). One established means of segregating the neuromodulatory effects produced by $\mathrm{NE}$, CORT, and their interactions, is pharmacologic fMRI (phMRI) (Patin \& Hurlemann 2011). A combination of phMRI with histoprobabilistic maps of the subregional architecture of the amygdala (Goossens et al. 2009; Hurlemann et al. 2008) revealed that blockade of $\beta$-noradrenergic receptors with the non-specific antagonist propranolol (40 mg po) desensitized the basolateral amygdala (BLA) (Hurlemann et al. 2010), which is consistent with behavioral data indicating that propranolol (40 mg po) eliminated a facilitation of declarative learning from facial feedback (Mihov et al. 2010). In contrast, enhancement of BLA reactivity with the NE re-uptake inhibitor (NARI) reboxetine (4 mg po) produced a response bias toward fearful faces (Onur et al. 2009). Together, these results suggest that increases in NE signaling may be essential for converting the BLA - an area of the brain controlled by powerful inhibitory circuits (Ehrlich et al. 2009) into a fear module (Onur et al. 2009). One interpretation of these findings is that phasic increases in endogenous NE signaling per se might be sufficient to code stimulus salience. However, because of its pivotal role in orchestrating fear memory acquisition and storage via $N$-methyl-D-aspartate (NMDA) receptor-mediated long-term potentiation (LTP) (Ehrlich et al. 2009), the BLA may be a locus of extensive GLU-NE interactions, such that observations of a reboxetine-induced increase in BLA signals may, in fact, support the GANE model.
In addition to rapid neuromodulatory effects mediated by $\mathrm{NE}$ per se, emotional arousal elicits heightened adrenal release of CORT, which feeds back on the amygdala and hippocampus via activation of mineralocorticoid and glucocorticoid receptors in these regions (de Kloet et al. 2005; McEwen et al. 2015). Experimentally, this endocrine response can be mimicked by exogenous administration of synthetic CORT (20-40 mg po), and studies based on this challenge not only have noted a desensitization of the amygdala during fear conditioning (Merz et al. 2010) and reward anticipation (Montoya et al. 2014), but also have detected timing-dependent changes in hippocampal memory functions. Specifically, when coinciding with declarative memory encoding, stress levels of CORT enhance long-term recall (Buchanan \& Lovallo 2001), whereas their occurrence during retrieval impairs performance (de Quervain et al. 2000).

Most important, endogenous CORT and NE signals do not act in isolation, and there is accumulating experimental evidence that coactivation of both systems under emotional arousal is crucial for facilitating amygdala-hippocampus interplay during declarative memory formation. The resultant advantage of privileged declarative encoding of salient stimuli, however, comes at the expense of reduced recall of preceding and following information. This periemotional amnesia is BLA as well as $\beta$-noradrenergic dependent (Hurlemann 2006; Hurlemann et al. 2005; 2007a; 2007b; Strange et al. 2003) and further amplified, in both magnitude and temporal extent, by combined prelearning administration of exogenous CORT (30 $\mathrm{mg}$ po) and reboxetine (4 mg po), thus suggesting synergistic NE-CORT interactions (Hurlemann 2008; Hurlemann et al. 2007a). The same pharmacologic intervention was found to induce a negative response bias toward fearful faces in the centromedial nucleus of the amygdala (CMA), an effect that was absent when CORT levels were augmented alone (Kukolja et al. 2008). Evidence indicates that response shifts mediated by CORT, NE, and their interactions are not restricted to the CMA, but propagate to interconnected areas including the dorsal striatum, which can be prevented by blockade of mineralocorticoid receptors with spironolactone (400 mg po) (Vogel et al. 2015b).

Collectively, these findings argue for a reallocation of neural resources as a function of CORT and NE coactivation under emotional arousal, hence enabling prioritized access to the salience network and memory stores. Obviously, this mechanism confers costs and benefits, evident in a larger devotion of amygdala-hippocampal resources during encoding (Kukolja et al. 2011) and deactivation of prefrontal cortex (PFC) (van Stegeren et al. 2010). It has been conceptualized that such co-occurrence of deficient top-down control from PFC and enhanced amygdala-hippocampus interactions under conditions of heightened CORT and NE release may result in hypermnesia for emotional events, which, when manifest in extreme forms, is pathognomonic of post-traumatic stress disorder (PTSD) (Hurlemann 2008). Converging support for this etiologic model comes from preclinical (Bryant et al. 2013) and clinical studies (Nicholson et al. 2014), both of which suggest that NE and CORT co-activation predisposes to the development of indelible memories. Future research addressing the mechanistic underpinnings of arousal-induced memory distortions in PTSD should, therefore, not only focus on neurotransmitter interactions between GLU and NE, as outlined by the GANE model, but also take the interplay of $\mathrm{NE}$ and endocrine players including CORT into perspective, which promotes stress-induced remodeling of neural architecture through (epi)genetic modifications as well as rapid non-genomic adaptations (de Kloet et al. 2005; McEwen et al. 2015). The latter include the non-genomic modulation of hippocampal glutamate transmission via activation of mineralocorticoid receptors (Karst et al. 2005), further illustrating the rapid susceptibility of memory functions to emotional arousal and stress. 
Commentary/Mather et al.: Norepinephrine ignites local hotspots of neuronal excitation

\section{Arousal-biased preferences for sensory input: An agent-centered and multisource perspective}

doi:10.1017/S0140525X15001855, e214

\author{
Kai Kaspar \\ Social and Media Psychology, Department of Psychology, \\ University of Cologne, 50931 Cologne, Germany. \\ kkaspar@uni-koeln.de \\ http://kai-kaspar.jimdo.com/
}

Abstract: I argue that the GANE (glutamate amplifies noradrenergic effects) model basically explains an arousal-based amplification of emotional stimuli, whereas effects on neutral stimuli indicate a contextualization process aiming to reduce stimulus ambiguity. To extend the model's validity, I suggest distinguishing between internal and external emotional sources, as well considering the stimulus valence and addressing age-related differences in attention and memory preferences.

Mather et al. beautifully describe the neuronal mechanisms likely to account for an arousal-based modulation of selectivity phenomena in attention and memory. In addition to previous emotioncognition models focusing on the competition between emotional and neutral stimuli, the GANE (glutamate amplifies noradrenergic effects) model also aims to explain how arousal resolves the competition between neutral stimuli. In this context, I propose a complementary perspective.

I argue that the GANE model explains mainly the mechanisms underlying the processing of emotional stimuli, whereas co-occurring effects on the processing of neutral stimuli may be interpreted as side effects of a contextualization process targeting the emotional stimulus. The empirical evidence Mather and colleagues present suggests that arousal-based amplification or inhibition of neutral input is heavily constrained by the spatiotemporal relationship between an emotional stimulus, which is the driving source of arousal, and neutral stimuli constituting the sensory context. I claim that effects of emotional stimuli on proximal neutral ones are a signature of stimulus contextualization in favor of a disambiguation of emotional stimuli. Indeed, for the perceiving agent, emotional stimuli are often characterized by significant ambiguity (cf. Duval et al. 2013). For example, the recognition of an arousing facial expression may be context dependent (cf. Barrett et al. 2011).

The valence and the discrete emotional category of an arousing stimulus are not completely inherent features of the stimulus, but they are also constituted by the context (including neutral stimuli) in which the arousing stimulus is embedded. Therefore, the processing of an emotional stimulus benefits from an amplification of salient neutral stimuli standing in an optimal spatiotemporal relationship to the emotional target stimulus. It appears beneficial for the human organism that emotional stimuli are not processed in isolation from rather neutral context information. Amplifying the processing of otherwise prioritized neutral stimuli in the presence of an emotional stimulus creates a context that facilitates the appropriate classification and encoding of the properties of the emotional stimulus. Indeed, the context seems to be routinely encoded during emotion perception (Barrett \& Kensinger 2010). In this sense, the arousal-based mechanisms outlined in the GANE model cannot be generalized to settings in which emotional stimulation is rather negligible. However, based on this perspective, two aspects may help to further improve the conceptual framework of the model and its validity:

First, I propose that the GANE model would benefit from a more explicit distinction between internally and externally located sources of emotional arousal (cf. Kaspar 2013; Kaspar \& König 2012). The current model addresses primarily the latter type, namely, sensory stimuli located outside of the perceiving agent, whereas internal forms of emotional arousal refer to the agent's current emotional/mood state. While the arousing power of external stimuli is tied to the stimuli and, hence, places tight spatiotemporal constraints for an amplification or inhibition of the neutral surrounding, arousal elicited and maintained by internal thoughts of the agent might be more easily linked to any neutral stimulus. Of course, an internally located source of emotional arousal might be elicited by an external source, but some residual arousal (i.e., mood) continues for a while after source offset. Indeed, excitation-transfer theories (Bryant \& Miron 2003; Zillmann 1983) propose that the residual arousal from a stimulus can be transferred to a subsequent stimulus, whereas the emotional valence of the stimuli may differ. If residual arousal can actually be tapped by a stimulus other than the original in this way (still to be shown), we can extend the validity of the GANE model by implementing a multisource approach to arousal-biased information processing.

Second, the model neglects the valence aspect of arousing sources being of central relevance, not only from the perspective of disambiguation tendencies. It seems that the arousal and valence ascribed to a stimulus are not completely independent features (cf. Kaspar \& König 2012), whereby negative (vs. positive) stimuli show a tendency toward higher arousal (Ito et al. 1998; Kim \& Hamann 2007). Thus, negative stimuli may have a higher potential to ignite neuronal hotspots and to fine-tune priority signals. This bias is plausible from an evolutionary perspective, as it is more prejudicial to miss a potential threat than to miss a potential reward. Thus, across different scenarios, external negative (vs. positive) stimuli may elicit stronger modulation effects on neutral stimuli being in an optimal spatiotemporal distance, whereas a negative (vs. positive) mood state might have more long-lasting effects because of more residual excitation.

Indeed, the location (internal vs. external) and valence of the arousing source have a critical influence on attention and memory processes. For example, younger adults showed an attentional preference and better memory performance for negative stimuli compared with positive stimuli, and this bias was more pronounced when participants had been in a positive (vs. negative) mood (Kaspar et al. 2015). Thus, internal and external sources of emotional arousal may show specific interactions depending on their valence. With respect to the GANE model, negative stimuli presumably have a higher likelihood to bias perception and memory when they are in strong contrast to emotional background noise within the perceiving agent. However, preferences for positive over negative information have also been reported for younger adults in specific circumstances (Becker \& Leinenger 2011; Parrott \& Sabini 1990; Schwager \& Rothermund 2013), indicating more complex mechanisms of the processing of emotionladen stimuli than delineated in an exclusive arousal-based model.

Finally, in this context, the GANE model suggests a brain-based explanation for the very reliable information processing bias toward positive (vs. negative) information in older adults (Reed et al. 2014). Mather and colleagues proposed that arousal may not increase selectivity similarly effectively among older adults because of agerelated changes in the locus coeruleus-norepinephrine system. If so, negatively valenced stimuli may gradually lose their high arousing potential across the life span, facilitating controlled attentional shifts toward positive stimuli at an older age (cf. Hahn et al. 2006; Knight et al. 2007). Thus, the GANE model adds a brainbased explanation for this age-dependent change in biased competition that is discussed mainly in terms of the socioemotional selectivity theory (Carstensen et al. 2003) emphasizing age-related changes in emotion-regulation motivation.

\section{Does arousal enhance apical amplification and disamplification?}

doi:10.1017/S0140525X15001867, e215

\section{E. Larkum ${ }^{\mathrm{a}}$ and W. A. Phillips ${ }^{\mathrm{b}}$}

${ }^{a}$ Neurocure Cluster of Excellence, Department of Biology, Humboldt University, 10117 Berlin, Germany; ${ }^{\mathrm{b} D i v i s i o n}$ of Psychology, School of Natural Sciences, University of Stirling, Stirling FK9 4LA, Scotland, United Kingdom. matthew.larkum@gmail.com wap1@stir.ac.uk 
Abstract: We summarize evidence that input to the apical tufts of neocortical pyramidal cells modulates their response to basal input. Because this apical amplification and disamplification provide intracortical mechanisms for prioritization, Mather and colleagues' arguments suggest that their effects are enhanced by noradrenergic arousal. Though that is likely, it has not yet been adequately studied. Their article shows that it should be.

Mather et al. argue that as arousal increases, things of high priority are perceived and remembered even better, whereas things of low priority are suppressed even more. Intracortical mechanisms for prioritization of selected signals are a prerequisite for this because the noradrenergic system provides only diffuse low-bandwidth innervation of neocortex, whereas the particular signals to be amplified or suppressed must be specified by locally specific interactions of high bandwidth. We therefore outline recent evidence for intracellular and microcircuit mechanisms by which signals are either amplified or suppressed within neocortex, prior to their further modulation by the noradrenergic system. We refer to those mechanisms as apical amplification (AA) and disamplification.

Evidence for AA is provided by patch-clamping studies showing that inputs to the apical tufts of pyramidal cells are integrated separately from inputs to their basal dendrites before being used to modulate the cell's response. Current models of neocortex, including noradrenergic effects, typically assume that pyramidal cells can be adequately thought of as point processors that simply sum all of their excitatory and inhibitory inputs and fire when that sum exceeds a threshold. The evidence for AA indicates that some pyramidal cells have not one, but two main sites of integration such that when apical and basal inputs coincide, intracellular calcium spikes initiated by a site of integration near the top of the apical dendrite amplify the cell's response to its basal inputs (Larkum 2013; Larkum et al. 1999; 2007; 2009).

The most studied mechanism by which AA is implemented in layer 5 cells is referred to as backpropagation-activated calcium spike firing (BAC firing). In addition to these two main integration sites, local integration takes place within both basal and tuft dendrites by the regenerative activation of $N$-methyl-D-aspartate receptors (NMDA spikes). AA may be fully implemented by NMDA spikes alone in supragranular neurons (Palmer et al. 2014), but even in subgranular neurons, NMDA spikes have an important influence (Larkum et al. 2009). Essential properties of these mechanisms are illustrated in Figure 1. Inhibitory interneurons that specifically target apical dendrites in layer 1 , such as Martinotti cells, produce disamplification, which suppresses amplification without inhibiting action potential output.

Though much of this work has been carried out in vitro, there are strong grounds for supposing that AA and disamplification apply to awake behaving humans (Phillips et al. 2015). Imaging studies of local dendritic NMDA spikes in awake behaving animals indicate the importance of such integrative intracellular processes in vivo (Cichon \& Gan 2015; Gambino et al. 2014; Grienberger et al. 2014; Lavzin et al. 2012; Palmer et al. 2014; Smith et al. 2013; Xu et al. 2012). These discoveries are well known to cellular neurophysiologists, but not yet to psychologists or cognitive neuroscientists. For a clear introduction to AA and disamplification and their relevance to cognitive function and theoretical neuroscience, see Phillips (2015).

Arousal releases norepinephrine (NE), that is, noradrenalin, which regulates the firing mode of layer 5 neurons (Wang \& McCormick 1993). Many new questions are raised by the possibility of interactions between $\mathrm{AA}$ and $\mathrm{NE}$ release in these and other neocortical neurons. First, are the effects of NE and AA synergistic, or do they simply sum in some quasi-linear way? Synergistic interactions between AA and mechanisms proposed in the GANE model seem likely because glutamate spillover will not spread from apical to basal dendrites. Spillover is intrinsic to the GANE model because of the non-synaptic component of NE release, and that implicates NMDA more than AMPA receptors. Local dendritic NMDA-spikes are also enhanced by glutamate spillover (Chalifoux \& Carter 2011). To see the possibility of synergistic interactions consider the case to which AA is most applicable, that is, where apical input is strong and basal input is present but weak. There would then be NE-dependent enhancement of depolarization in the apical tuft but not in the basal dendrites. That would increase the effect of AA on cellular output while maintaining the need for basal input to initiate axonal spiking. Second, how are NE-receptor subtypes distributed across regions, layers and subcellular compartments, and is that compatible with the modulatory role proposed for tuft inputs? An explicit focus on intracellular and microcircuit mechanisms in theories of arousal requires answers to these questions.
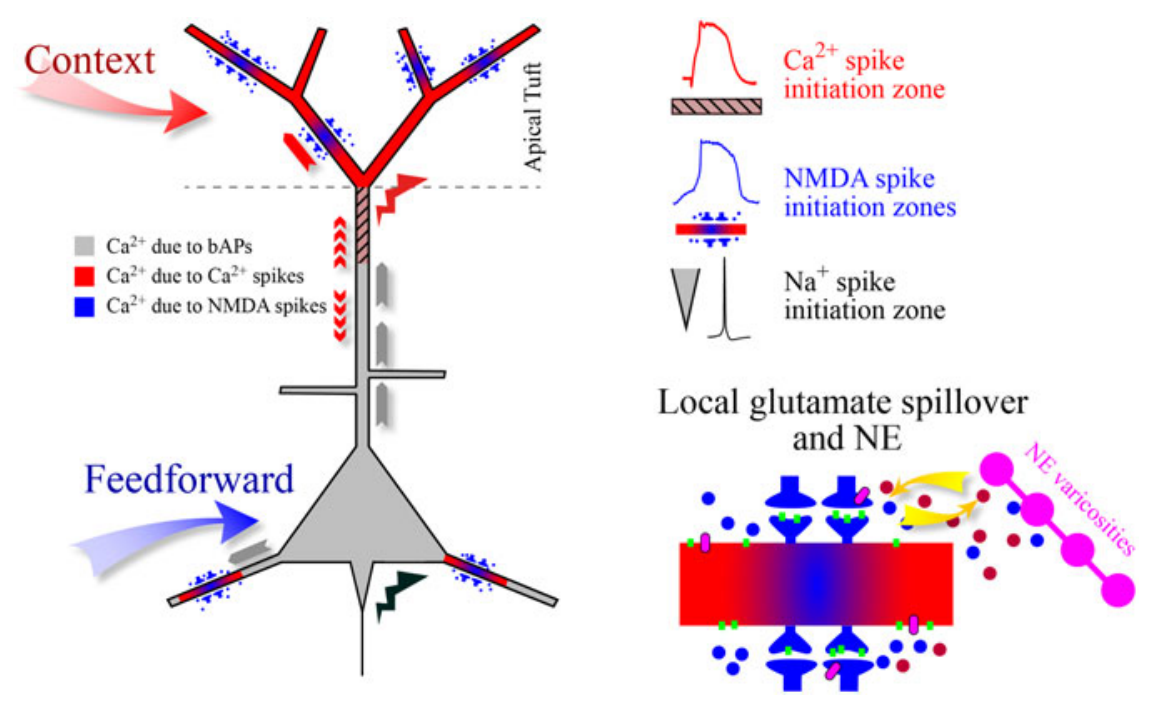

Figure 1 (Larkum \& Phillips). Dendritic spikes in neocortical pyramidal neurons. Apical tufts of pyramidal neurons receive inputs from diverse sources. Calcium currents, and thus synaptic plasticity, depend on backpropagating action potentials (bAPs, gray), apical dendritic calcium spikes (red) and NMDA spikes (blue). NMDA spikes require both local depolarization and glutamate (blue dots) and are enhanced by glutamate spillover to extrasynaptic NMDA receptors (green squares). Norepinephrine (maroon dots) interacts with glutamate in a feedback process hypothesized to enhance post-synaptic excitability. 
Third, will studies of interactions between AA and NE cast light on the putative role of AA in regulating states or levels of consciousness (Bachmann \& Hudetz 2014; Meyer 2015; Phillips 2015)? It seems likely that they will. Fourth, do previous studies under-estimate the extent of AA because they do not ensure appropriate levels of noradrenergic input? This is clearly relevant to in vitro studies or under anesthesia, but, Mather and colleagues's hypotheses imply that local phasic arousal needs to be considered as well as tonic arousal when studying awake behaving animals. Finally, are working memory capabilities dependent upon specialized interactions between $\mathrm{NE}$ and $\mathrm{AA}$ in dorsolateral prefrontal cortex (Arnsten et al. 2012)?

Much intracellular, electrophysiological, cognitive, and computational research is required to answer such questions. If that shows noradrenergic enhancement of AA and disamplification, then that will strengthen and broaden both the GANE model and our understanding of the role of intracellular computations in mental life. If not, then we will need to discover why not. Thus, the target article opens the door to a wide array of issues concerning interactions between noradrenergic arousal and prioritization within the neocortex by AA and disamplification. These issues may well be crucial to our understanding of relations between brain and behavior.

\section{Emotional memory: From affective relevance to arousal}

\author{
doi:10.1017/S0140525X15001879, e216
}

Alison Montagrin ${ }^{\mathrm{a}, \mathrm{b}, \mathrm{c}}$ and David Sander
a,b
'Swiss Center for Affective Sciences, University of Geneva, 1205 Geneva,
Switzerland; ${ }^{\mathrm{b}}$ Department of Psychology, University of Geneva, 1205 Geneva,
Switzerland; ${ }^{\mathrm{c}}$ Department of Basic Neurosciences, University of Geneva, 1205
Geneva, Switzerland.
alison.montagrin@ @unige.ch david.sander@unige.ch
http://cms.unige.ch/fapse/EmotionLab/Members/alison-montagrin/index.
php
http://cms.unige.ch/fapse/EmotionLab/Members/david-sander/index.php

Abstract: Arousal is typically conceived as a key component of emotional response. We describe here the psychological processes thought to elicit arousal - in particular, the processes involved in the appraisal of affective relevance. The key role of relevance in attentional and memory processing, and its links with arousal, is discussed with respect to the GANE (glutamate amplifies noradrenergic effects) model described by Mather et al.

Mather et al. provide an innovative and integrative model aimed at explaining, at the neural level, how arousal can both enhance and impair cognitive processing, such as perception and memory. The glutamate amplifies noradrenergic effects (GANE) model proposed by the authors accounts for results indicating that increased norepinephrine under arousal affects prioritization of information processing, for example, enhances memory for salient information at the expense of mundane information. Mather et al. consider arousal to be the critical factor that amplifies the perception of emotional stimuli while impairing the perception of other concurrent stimuli, as has been proposed in the arousal-biased competition (ABC) theory (Mather $\&$ Sutherland 2011). Mather et al. are particularly interested in analyzing the effect of selectivity in the perception and memory of emotional stimuli under arousal; however, the construct "arousal" is not conceptually clear if one considers how it is used in the affective sciences literature, especially concerning theories of emotion. More generally, the relationship between emotion and arousal is far from being consensual, notably when it comes to modeling the psychological mechanism that elicits different types of arousal (see Sander 2013). For example, Frijda (1986) distinguished among three response systems that embrace the construct of arousal or activation: autonomic arousal, electrocortical arousal, and behavioral activation. Traditionally, theories of emotion refer to arousal mainly in terms of either (1) the consciously felt activation dimension of the experienced affect or (2) the bodily reaction during an emotional episode, specifically the sympathetic nervous system. Dimensional theories propose that any experienced affect can be described as a particular feeling on a continuum in terms of an activated (e.g., as in surprise) or deactivated (e.g., as in boredom) state (Feldman 1995; Russell 1989). The bodily reaction can be measured in terms of a psychophysiological state driven by the activation of the sympathetic nervous system. Emotion researchers typically use the construct of arousal to refer to the activation of the sympathetic nervous system, and physiological measures (e.g., skin conductance response or pupil dilation) are often used as measures of emotion intensity, as they are also considered to mirror subjective affective ratings (see Bradley et al. 2008; Lang et al. 1993).

The effect of arousal on performance has been revealed in various research domains and is consistent with the model proposed by Mather et al., suggesting that - under arousal-neutral stimuli with a high priority are processed preferentially.

The priority factor is essential to memory enhancement. Indeed, some have proposed that arousal per se is not sufficient to explain memory improvement for neutral information (Reisberg \& Heuer 2004). For example, a study that manipulated arousal by injection of adrenalin versus saline showed no difference in the results of memory performance for verbal descriptors (e.g., name, occupation) presented previously with neutral faces, although heart rate and skin conductance increased in the group with adrenalin injections (Christianson \& Mjörndal 1985). The information presented did not differ in terms of priority. This notion of priority, especially when related to the well-being or major concerns of the individual, is considered to be key in explaining emotion elicitation. Indeed, appraisal theories of emotion suggest that stimuli that are appraised as relevant for an individual's concerns (e.g., that relate to goals, needs, and values) are typically those that have the competency to elicit an emotional response by driving changes in action tendencies, in expressions, in the peripheral nervous system, and in the conscious feeling (e.g., Sander et al. 2005). With respect to arousal, a conceptually interesting advantage of such models is that an explanation for the elicitation of arousal is proposed: only concern-relevant events elicit arousal. Evidence indicates that stimuli that are appraised as concern relevant not only elicit such emotional responses, but also are prioritized in attention (see Pool et al. 2015). For example, a recent meta-analysis revealed that the magnitude of the attentional bias for positive stimuli varies as a function of arousal, but also that this bias is significantly larger when the stimulus is relevant to specific concerns (e.g., hunger) of the participants compared with other positive stimuli that are less relevant to the participants' concerns (Pool et al. 2015). Interestingly, in this meta-analysis, when arousal and relevance moderators were tested by statistically controlling their respective variances, only relevance remained a significant predictor of the magnitude of this bias in emotional attention. Emotional attention is a process that has been suggested to strongly rely on the amygdala (Vuilleumier 2005a), a region that has also been suggested to be critical for the process of appraised relevance (e.g., Cunningham \& Brosch 2012; Sander et al. 2003) and of importance in promoting memory consolidation, as described by Mather et al. (see Roozendaal \& McGaugh 2011 for a review).

Studies supporting the ABC theory (e.g., Sutherland \& Mather 2012) and GANE models are based largely on experimental manipulation showing that high-priority neutral information under external arousal is enhanced in memory (as compared with low-priority neutral information). Showing that goal-relevant events, which are intrinsically neutral, are processed preferentially might extend the model proposed by Mather et al. For example, the goal relevance hypothesis of memory facilitation has been 
tested in a gamelike study in which the goal of the participant was to win points. Participants showed better memory performance for initially neutral items that signaled a gain (i.e., goal-conducive items) than for initially neutral items that were goal irrelevant (Montagrin et al. 2013).

Our suggestion is that events that are relevant to one's goals elicit an arousal response, capture attention, and facilitate memory. A fascinating research topic, for which both the GANE model and the $\mathrm{ABC}$ theory can be particularly well articulated with appraisal theories of emotion, is to understand the causal relationships between relevance detection, arousal, attention, and memory.

\section{Effect of arousal on perception as studied through the lens of the motor correlates of sexual arousal}

\section{doi:10.1017/S0140525X15001880, e217}

\section{Harold Mouras}

EA 7273, Centre de Recherche en Psychologie: Cognition, Psychisme et Organisations, UFR de Sciences Humaines Sciences Sociales et Philosophie, Departement de Psychologie, Universitaire de Picardie Jules Verne, F-80000 Amiens, France.

hmouras@gmail.com harold.mouras@u-picardie.fr

Abstract: The study of sexual arousal is at the interface of affective and social neurosciences. Recent results regarding the motor correlates of sexual arousal demonstrating an early freezing response are in perfect accordance with the GANE (glutamate amplifies noradrenergic effects) model's sustaining the double role of the arousal dimension on emotional processing.

During recent decades, sexual affiliation has been an exponentially explored functional context within socioaffective neuroscience, especially with respect to the motivational component of inter-attraction, which can be either positive or negative within social relationships. As appearing in international databases, sexual visual stimuli are often reported as the most arousing stimuli and are therefore ideal to increase knowledge of the influence of the arousal dimension on the neural (central and peripheral) and psychological correlates of emotional information processing, which is at the center of the theory presented by Mather et al.. Here, we focus on recent results regarding the motor correlates of visual sexual information corroborating the complex modulatory role of arousal as developed in the GANE (glutamate amplifies noradrenergic effects) theory.

Within the framework of sexual behavior, emotion can be conceptualized partly as an action disposition characterized by a context-dependent (e.g., approach vs. avoidance) behavioral component, which may be mediated by automatic responses (Campbell et al. 1997; Panksepp \& Biven 2012). In that sense, emotion should influence several steps of the motor response (Bradley et al. 1992; Helbig et al. 2011; Williams et al. 1996) by inducing an approach behavior to what promotes our well-being and our survival and an avoidance behavior in response to painful experiences (Elliot \& Covington 2001). Albeit recent, most neuroimaging studies of sexual arousal made central in their theoretical model a motivational component. Several
A

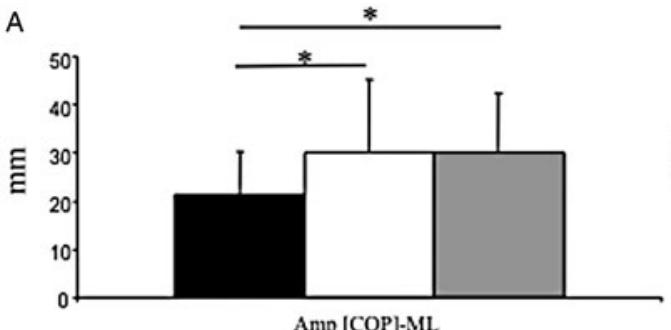

Amp [COP]-ML

sexually explicit clips

C

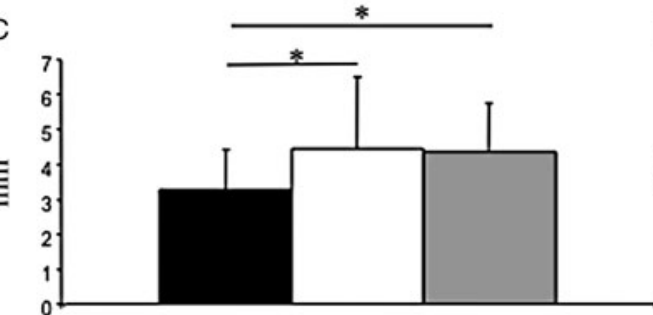

SD [COP]-ML.

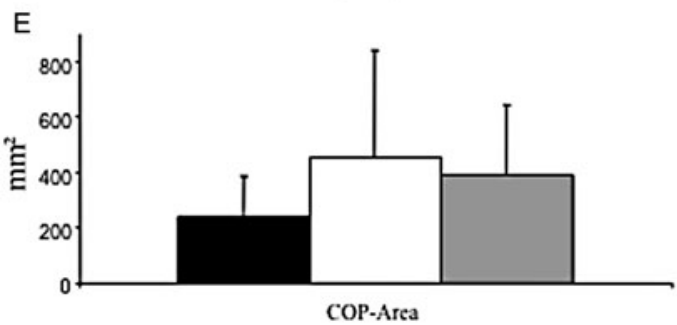

COP-Area
B

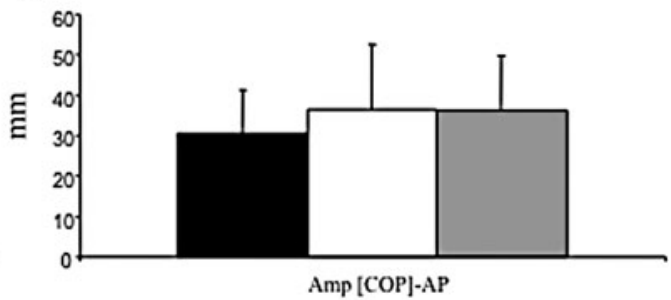

neutral documentary clips

humorous clips

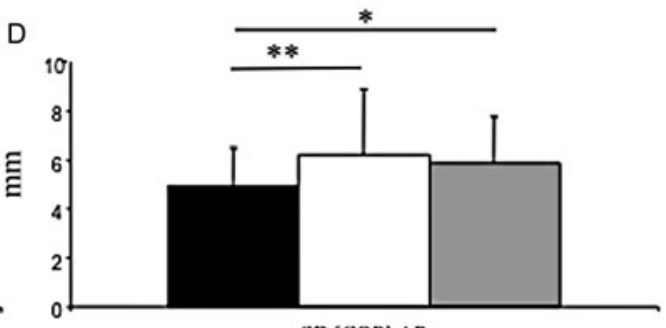

SD [COP]-AP

Figure 1 (Mouras). Means and standard deviations of postural indices as a function of the stimulus. (A) Amplitude of the sway of the center of pressure (COP) in the mediolateral direction (Amp [COP]-ML). (B) Amplitude of the sway of the COP in the anteroposterior direction (Amp [COP]-AP). (C) Standard displacement of the COP in the mediolateral direction (SD [COP]-ML). (D) Standard displacement of the COP in the anteroposterior direction (SD [COP]-AP). (E) Area encompassed by displacements of the COP (COP-area). ${ }^{*} p<0.05,{ }^{* * *} \mathrm{p}<0.01$ when comparing stimuli. (From Mouras et al. 2015). 
incentive motivation theories state that approach behavior is activated by appropriate incentives (Agmo 1999; Bindra 1974; Singer $\&$ Toates 1987). This theoretical framework is supported by recent results indicating that, in sexual conditions, behavioral responsiveness and interest (Both et al. 2004) and spinal tendon reflexes (Both et al. 2005) increase linearly with the arousal level of the stimuli.

Posture is a good index of motor correlates of emotional information processing and can be quantified by determining the body's center-of-pressure (Gurfinkel 1973) displacements. Previous studies reported the influence of emotion on decisionmaking processes (Damasio et al. 1996) through emotional biasing of action selection, which might indicate the functioning of a Pavlovian system that innately regulates specified responses to reward- or punishment-predictive stimuli (Ly et al. 2014). This is supported by numerous studies reporting, respectively, behavioral activation versus inhibition in response to reward versus punishment (Cavanagh et al. 2013; Crockett et al. 2009; Guitart-Masip et al. 2011; 2012; 2014).

Contrary to the primary hypothesis of an approach-type behavior, our recent study (Mouras et al. 2015) demonstrated a freezing-type response to sexually explicit stimuli (Fig. 1).

These results were quite surprising - and in accordance with the GANE theory's interpretation of the effect of arousal on perception and memory. Previous studies reported a freezing strategy in response to aversive visual images (Facchinetti et al. 2006; Hillman et al. 2004; Stins \& Beek 2007) or a defense-type response to unpleasant compared with both pleasant and neutral videos (Hagenaars et al. 2014b). Interestingly, Horslen and Carpenter (2011) argued that arousal only modulated the freezing strategy. Hagenaars et al. (2014a) reported that freezing (a) is usually considered a threat-related defense strategy and (b) could be similar to immobility occurring in orienting or behavioral inhibition. The temporal dimension of the motor correlates may be central as demonstrated by early (1-2 s after stimulus onset) freezing behavior in response to unpleasant films (Hagenaars et al. 2014a) that would be associated with an optimal body position for concealment from the predator (McNaughton \& Corr 2004). Therefore, sexual arousal could be partly associated not only with positive emotions, but also with a certain anxiety in accordance with a freezing response to angry faces and related to anxiety (Roelofs et al. 2010). Facchinetti et al. (2006) reported that body sway reductions were observed in response to both unpleasant and pleasant pictures and suggested that "baby and family pictures may have elicited (for subjects) a predisposition to social bonding and that the pre-activation of muscles involved in the anterior-posterior displacement could reflect preparation for processes like attachment and reduction of social distance."

\section{What do we GANE with age?}

doi:10.1017/S0140525X15001892, e218

\section{Matthew R. Nassar, ${ }^{a}$ Rasmus Bruckner,, ${ }^{b, c}$ and Ben Eppinger ${ }^{\text {d,e }}$}

${ }^{a}$ Department of Cognitive, Linguistic, and Psychological Sciences, Brown University, Providence, RI 02912; ${ }^{\mathrm{b} D e p a r t m e n t ~ o f ~ E d u c a t i o n ~ a n d ~ P s y c h o l o g y, ~}$ Freie Universität Berlin, Berlin, Germany; ${ }^{\circ}$ International Max Planck Research School on the Life Course (LIFE), Berlin, Germany; ${ }^{\mathrm{d} D e p a r t m e n t ~ o f ~}$ Psychology, TU Dresden, Dresden, Germany. ${ }^{\mathrm{e}}$ Department of Psychology, Concordia University, Montreal, H4B 1R6 Quebec, Canada matthew_nassar@brown.edur.bruckner@40fu-berlin.de ben.eppinger@concordia.ca

Abstract: Mather and colleagues provide an impressive cross-level account of how arousal levels modulate behavior, and they support it with data ranging from receptor pharmacology to measures of cognitive function. Here we consider two related questions: (1) Why should the brain engage in different arousal levels? and (2) What are the predicted consequences of age-related changes in norepinephrine signaling for cognitive function?

Mather and colleagues have developed an impressive theoretical model linking arousal-mediated changes in cognition to the local signaling dynamics at the axon terminals of noradrenergic locus coeruleus (LC) projections. The authors provide compelling evidence for how this link between biology and cognition is made; however, they leave open a key question: Why should the brain undergo fluctuations in arousal that influence information processing? Answering this question is important not only for appreciating the intricate biological design proposed by Mather and colleagues, but also for understanding the contexts in which such a design would be maladaptive. Here we explore the why question and speculate that healthy aging may constitute one such maladaptive context.

So, if the brain has a good system for prioritizing sources of information, why should it ever be turned down? One possible answer is that prioritized sources of information are often imperfect predictors of behaviorally relevant variables. In such cases, inferring the variable of interest (e.g., the best foraging location) requires combining probabilistic sensory information (e.g., the color of the berry bushes on a distant hill) with currently held beliefs (how many berries are expected based on past experience). The relative contributions of these factors should be determined by the precision with which they predict the behaviorally relevant variable; thus, sensory information should be discounted when prior expectations are strong (e.g., the forager has recently counted the berries on the bush) or when sensory evidence is weak (e.g., the color of the bush is a bad predictor of caloric yield). In either circumstance, amplifying the prioritized sensory information would disrupt inference by allowing poor sensory cues to overwhelm precise internal expectations. There is some evidence to suggest that arousal levels are decremented under such conditions. Pupil diameter, a marker for arousal, is large during periods of uncertainty and constricts as expectations become more reliable (Lavín et al. 2014; Nassar et al. 2012; Nieuwenhuis et al. 2011; Preuschoff 2011). Information that is inconsistent with expectations drives sharp increases in arousal that appear to affect the relative influence of new observations on behavior (Lavín et al. 2014; Nassar et al. 2012; Nieuwenhuis et al. 2011; Preuschoff 2011). These data suggest that decrements in arousal, likely implemented through reductions in the firing of LC neurons, may serve an important role in optimal inference (Joshi et al. 2016). In particular, one role of low arousal levels might be to protect strong internal predictions from prioritized but potentially distracting information.

Another normative justification for reducing the influence of priority maps is that under some conditions, it is useful to explore alternatives to the current course of action that might provide better long-run returns. For example, information about a known source of reward (e.g., the berry bush on the hill) might be prioritized over other potential sources of reward that are yet to be discovered (e.g., an apple tree that only recently began to bear fruit). Thus, reducing the influence of priority maps could provide an incentive to explore non-prioritized inputs. Exploring potential alternative reward streams becomes important as the known source of reward is depleted, particularly if there is sufficient time to capitalize on any knowledge gained in the exploratory process (Wilson et al. 2014). There is some evidence to suggest that shifts from exploiting a known source of reward to exploring alternative options are accompanied by a shift from a phasic (stimulus evoked) mode of LC firing to a high tonic mode (Aston-Jones \& Cohen 2005; Jepma \& Nieuwenhuis 2011). Thus, fluctuations in arousal might allow for an effective navigation of the trade-off between exploitation and exploration in addition to optimizing inference.

The biological mechanism proposed by Mather and colleagues suggests that the optimization of inference and exploration 
through fluctuations in arousal may be highly sensitive to the state of the LC-norepinephrine (NE) system and its biophysical components. There is some evidence for dysregulation of this system over the course of healthy aging. Findings from histologic post-mortem studies point to a substantial cell loss in the LC with age (Chan-Palay \& Asan 1989; Grudzien et al. 2007; Manaye et al. 1995). Moreover, neuronal density in LC is strongly related to cognitive decline in the period before death (Wilson et al. 2013). These findings seem to line up with recent in vivo structural magnetic resonance imaging findings that point to neuromelaninrelated magnetic resonance signal loss with age (Shibata et al. 2006). Taken together, these findings suggest that aging is associated with substantial structural changes in the LC, which are associated with cognitive decline.

One potential cause of age-related cognitive decline could be that this pattern of changes in the NE system disrupts optimal inference. In particular, lower levels of NE could prevent the positive feedback of glutamate on NE release from achieving high enough NE levels to activate low-affinity $\beta$-adrenoceptors proposed by Mather and colleagues. This could lead to a suppression of high-priority signals, even at high arousal levels associated with uncertainty, when new information should be highly influential on behaviorally relevant beliefs. Consistent with this notion, older adults show selective behavioral impairments at learning under conditions of uncertainty, the same conditions that typically drive increased arousal and increased influence of new information on learning in younger participants (Eppinger et al. 2008; 2011; Nassar et al. 2012; 2016). The cause of this learning impairment has not been directly linked to the function of the LC-NE system to date, but in light of the biological link provided by Mather and colleagues, it should be explored in the very near future.

Changes in NE functioning with age may also affect the ability to mediate exploration and exploitation in older adults. In particular, reduced NE levels may prevent phasic signals from activating $\beta$-adrenoceptors, even in regions where signals are prioritized through association with an exploitative action. This would reduce the contrast between exploitative and exploratory action representations and shift behavior toward a more exploratory regime. Interestingly, increased choice variability, which can be used as a strategy for random exploration, is enhanced in older adults across a wide range of tasks (Garrett et al. 2013). Future work should focus on animal models where the mechanisms for age-related changes can be explored to the level of detail specified by Mather and colleagues.

\section{"What have we GANEd?" A theoretical construct to explain experimental evidence for noradrenergic regulation of sensory signal processing}

\author{
doi:10.1017/S0140525X15001909, e219
}

\section{Rachel Navarra and Barry Waterhouse}

Department of Cell Biology and Neuroscience, Rowan University School of Medicine, Stratford, NJ 08084.

navarra@rowan.edu waterhouse@rowan.edu

Abstract: The GANE (glutamate amplifies noradrenergic effects) theory posits a mechanism for amplifying noradrenergic modulatory actions and enhancing the processing of high-priority sensory signals for immediate or future experience-guided action. This theoretical construct is thought provoking with respect to the central processing of high-priority versus low-priority stimuli, but it requires some refinement to account for physiological fluctuations in NE efflux as a function of naturally occurring transitions in behavioral state and the experimentally observed phenomena associated with noradrenergic regulation of sensory signal transfer.
Mather et al. have proposed an intriguing theory to explain how norepinephrine (NE) release and subsequent noradrenergic modulatory actions are focused in neural circuits by concomitant "priority" stimulus-driven release of glutamate. In doing so they confront a question that has perplexed the field for some time, that is, how to account for selectivity of signal processing in noradrenergic terminal fields and focused perception of salient events when tonic discharge from the broadly projecting NE-containing nucleus locus coeruleus (LC) is elevated, as would occur during generalized arousal. Here we focus on how the theory applies to NE modulation of sensory signal processing. Given the results of four decades of published work, we would expect increases in LC-NE output to promote enhanced neuronal and neural network responses to sensory-driven afferent inputs (Berridge \& Waterhouse 2003), actions that have been linked to improved performance of sensory-guided behavioral tasks (Aston-Jones et al. 1999; Rajkowski et al. 2004).

Until recently, conventional wisdom was that the LC-NE efferent network was broadly distributed from a relatively small number of brainstem neurons with homogeneous physiological properties. Given this, the presumption was that NE was released uniformly and simultaneously across all terminal fields within the forebrain, cerebellum, and spinal cord for as long as LC is discharging, either tonically or phasically. If that were the case, neuronal and neural circuit responsiveness to sensory driven afferent inputs would be increased throughout the central nervous system without any bias in favor of one sensory signal versus another. If responsiveness to synaptically driven inputs is elevated everywhere and for every modality, what has been gained? Is there a way for the LC-NE system to selectively differentiate sensory signals from the constant stream of information that is presented to the nervous system from the periphery? Mather and colleagues' GANE (glutamate amplifies noradrenergic effects) theory is timely in so far as it appropriately confronts these issues.

An idea similar to the current theory was suggested by Marrocco et al. (1987) after they observed a correlation between catecholamine release in monkey visual cortex and coincident light-evoked activity in geniculostriate projections to ocular dominance columns. These authors postulated a local interaction between NE fibers and geniculostriate afferents: one that created a local hotspot for NE release within the visual cortex and, thus, preferentially promoted modulation of synaptic transmission at this site. Akin to Marrocco and colleagues' proposal, the GANE theory argues that locally released glutamate provides the means for amplifying release of $\mathrm{NE}$ from tonically or phasically active LC-NE fibers.

The GANE theory accounts for many, but not all of the welldocumented attributes and operational capacities of the LC-NE system, particularly those demonstrated in sensory networks. The authors exhaustively reviewed an extensive literature including many reports that support the core of their proposal: a positive feedback mechanism whereby synaptic release of glutamate amplifies NE release from nearby noradrenergic axons and results in enhanced responsiveness of neurons and glia to glutamate neurotransmission at this local site of interaction. The process relies on a delicate balance and interplay between receptor-mediated actions that are triggered as extracellular concentrations of $\mathrm{NE}$ and glutamate change. The temporal and spatial dynamics of these interactions are postulated, but experimental evidence to support the details of these mechanisms is lacking. For example, to date, the extracellular tissue concentrations of $\mathrm{NE}$ that yield the range of modulatory actions demonstrated in vivo and in vitro have been only crudely approximated based upon the results of microdialysis studies (Berridge and Abercrombie 1999; Florin-Lechner et al. 1996). As illustrated in many studies, LC-NE modulatory effects are expressed according to an inverted-U dose-response curve, rising to optimal facilitation of cellular and behavioral events as LC-NE activity increases and then falling to suppression of neuronal responsiveness and disrupted task performance as $\mathrm{NE}$ concentrations and LC 
discharge increase further (Aston-Jones et al. 1999; Devilbiss \& Waterhouse 2000). At what point along this inverted-U function is the glutamate-NE interaction operating? At some level of glutamate release, the facilitating actions of NE would be expected to diminish along the right side of the function.

Other aspects of LC-NE action require attention in the GANE model. For example, GANE relies on $\beta$-receptor mediated modulation of excitatory synaptic transmission and minimizes a role for $\alpha_{1}$-receptors, despite evidence in sensory circuits that $\alpha_{1}$-receptor activation augments postsynaptic responses to excitatory inputs (Mouradian et al. 1991; Nai et al. 2010; Rogawski \& Aghajanian 1982). In this same vein, $\beta$-receptor activation has been reported to enhance postsynaptic responses to $\gamma$-aminobutyric acid (GABA) (Cheun \& Yeh 1992; Waterhouse et al. 1982). The latter mechanism could account for the enhanced lateral inhibition that is invoked as a means of establishing additional contrast between a priority stimulus-driven hotspot and its tissue surround. Evidence for increased lateral inhibition and focusing of the zone of neural excitation created by prioritized inputs can be found in studies that examined the impact of the LC-NE system on off-beam inhibition in the cerebellar cortex (Moises et al. 1983) and receptive field properties of visually responsive neurons (Waterhouse et al. 1990). Finally, a "gating" effect of the LC-NE system on otherwise subthreshold synaptic inputs has been demonstrated in multiple brain circuits (Waterhouse et al. 1988). This action of NE would serve to recruit additional neurons into a sensory response pool, as opposed to suppression of hotspot surround activity.

In summary, the model works reasonably well in support of circumstances in which a noradrenergically innervated circuit is called on to process behaviorally imperative information that should be prioritized for immediate response and/or accentuated for future retrieval and experience-guided action, for example, fear-related or reward-generating stimuli. This theoretical construct is extremely valuable in providing the platform for generating testable hypotheses about glutamate-facilitated noradrenergic transmission in sensory and cognitive circuits that process potentially imperative stimuli. However, it is well to remember that much of the evidence for NE modulatory actions in sensory circuits has been demonstrated in anesthetized or controlled waking conditions where stimuli are behaviorally irrelevant. How does GANE account for bottom-up differentiation and prioritization of sensory signals in the absence of behavioral relevance? We applaud the model but look forward to resolution of how GANE integrates with conventional NE modulation to differentiate signals amidst the constant stream of incoming information from the sensory surround at both early-stage sensory relays and areas of higher-order sensory/cognitive integration.

\section{Competition elicits arousal and affect}

\section{doi:10.1017/S0140525X15001910, e220}

\section{R. Hans Phaf}

Amsterdam Brain and Cognition Center and the Brain and Cognition Group at the Department of Psychology, University of Amsterdam, 1018 XA Amsterdam, The Netherlands.

r.h.phaf@uva.nl

\begin{abstract}
The emotion-cognition integration in Mather et al. can be extended by specifying the relationship between competition and arousal in the reverse direction. According to affective monitoring, competition raises arousal, which, when sustained, results in negative affect, evoking theta oscillations, and when resolved, in positive affect, evoking gamma oscillations. Competition should be considered a core process in both cognition and emotion.
\end{abstract}

Competitive processes in the brain have the potential to account for a much larger range of behavioral functions than only attention and memory. Mather et al. successfully account for enhancing and impairing effects of arousal (and norepinephrine [NE]) on selective attention and memory in terms of neural competition, but still sometimes mix the brain metaphor with the "steam engine" metaphor. If competition is envisaged as mutual inhibition between neural nodes, there is no further need to borrow conservation laws from nineteenth-century physics and invoke limited resources to explain interference. Whereas arousal effects are thus predominantly analyzed in terms of neural processes, the source of arousal and emotion is discussed here only on a behavioral level. The analysis can be extended by also grounding arousal and affect in processes of neural competition.

Emotion research abounds in examples where modulatory influences of affect on attention and memory appear to be reciprocal, in that a similar, but not affectively laden, manipulation of attention or memory is able to elicit affect (e.g., Dreisbach \& Fischer 2012; Phaf \& Rotteveel 2005; Rotteveel \& Phaf 2007; Srinivasan \& Hanif 2010). Most relevant to the present discussion may be studies demonstrating distracter devaluation, and target appreciation, in attentional filtering tasks (Goolsby et al. 2009; see also Raymond et al. 2003). The present authors explain target selection in attentional tasks by biased competition (Desimone \& Duncan 1995; Duncan 1996; see also Phaf et al. 1990), so the appreciation and devaluation may also follow from competitive processes. Phaf and Rotteveel (2012) have argued that affect is the consequence of competition (i.e., conflicting neural representations [cf. Murre et al. 1992), and that negative affect arises when it is sustained, but positive affect when it is quickly resolved. Fluent, competition-less processing in itself is not sufficient to raise positive affect (but see Fazendeiro et al. 2007). The common denominator in both positive and negative affect is the initial competition, which may thus correspond to arousal. The largely similar effects of positive and negative arousal (e.g., Sutherland \& Mather, under review) may thus result from this initial phase of competition.

Gamma and theta oscillations are involved but not well integrated by the authors in their competitive framework. Such integration, however, serendipitously emerged from our evolutionary simulations (Heerebout \& Phaf 2010a; 2010b). Random variation combined with selection of the fittest individuals led to the development of both competition and oscillations in neural networks that controlled agents roaming an artificial environment. The fitness measure combined the amount of food gathered and the time the agent managed to escape from predators. The serendipitous emergence of oscillations coincided with a near doubling of fitness, indicating that they were very functional to the agents. In fact, the same feedback loops between excitatory and inhibitory nodes developed autonomously in the evolutionary simulations as were suggested in Mather et al. Heerebout and Phaf (2010a) investigated the behavior of these agents and found that the function of oscillations was complementary to that of competition. Competition enables the selection of representations, and oscillations allow for a reset of winners (i.e., switching of representations).

Attentional flexibility is more functional with positive than with negative affect. When searching for food, it is highly adaptive to be able to quickly shift attention toward an approaching predator. When running from a predator, it is highly maladaptive to switch attention to some palatable food (i.e., "it is better to miss dinner than to be dinner"). We hypothesized that low-frequency, presumably theta, oscillations are evoked by competition, whereas high-frequency, presumably gamma, oscillations arise when the competition is solved. Similar to arousal (and NE), the former increase selectivity and narrow attentional focus, whereas the latter enhance switching between representations and attentional flexibility (Bauer et al. 2009; Heerebout et al. 2013). Crucially, in this view, theta oscillations (i.e., resulting from competition) precede gamma oscillations and may thus be the most primary reflection of arousal. According to this framework, without competition there is no arousal, theta synchrony (i.e., negative affect), or eventual gamma synchrony (i.e., positive affect). 
Doubtlessly, the functioning of biological networks is more complex than in these simple models, which, however, provide a useful starting point for the integration of such disparate functions as attention, memory, arousal, affect, and neural oscillations.

Mather et al. attempt to break down the traditional distinction between hot emotional and cold cognitive processes by discussing emotional modulation of cognition. Not only, however, are there modulatory effects of arousal and emotion on attention and memory for neutral material, but also the elicitation of arousal and affect from the processing of neutral material can be observed (e.g., from novelty, conflict in classic Stroop [Phaf \& Rotteveel 2012]). The reverse modulation of emotion by cognition further strengthens such attempts at integration. With respect to neuronal organization, this must imply that specialized emotional and cognitive modules should not be distinguished, but that a more distributed picture of dynamic coalitions of multifunctional brain regions emerges (cf. Pessoa 2008). Not only does neural competition seem an obvious candidate for the regulation of these processing streams, but also it can serve to account in an almost mechanical fashion for both emotional and cognitive sub-processes. Unfortunately, at the end, Mather and coauthors seem to qualify this role again by arguing that the GANE model does not require competition to be a fundamental mechanism, but that it applies to whatever priority mechanism is acting. If this refers to the resources from the steam engine metaphor, it is unclear how they can also account for the elicitation of arousal and affect. To paraphrase the beginning of the authors' conclusion: Competition is the core of what allows both our emotional and cognitive systems to function effectively.

\section{Adaptive memory systems for remembering the salient and the seemingly mundane}

\section{doi:10.1017/S0140525X15001922, e221}

\section{Maureen Ritchey, ${ }^{a}$ Vishnu P. Murty, ${ }^{b}$ and Joseph E. Dunsmoor ${ }^{\mathrm{C}}$}

${ }^{a}$ Center for Neuroscience, University of California, Davis, CA 95618; ${ }^{\mathrm{b}}$ Department of Psychiatry, University of Pittsburgh, Pittsburgh, PA 15213; 'Department of Psychology, New York University, New York, NY 10003. meritchey@ucdavis.edu murtyv@upmc.edu

joseph.dunsmoor@nyu.edu

\begin{abstract}
In an adaptive memory system, events should be prioritized in memory based on their own significance, as well as the significance of preceding or following events. Here we argue that tag-and-capture models complement the GANE (glutamate amplifies noradrenergic effects) model by describing a mechanism that supports the transfer of memory benefits from one event to the next.
\end{abstract}

Imagine you are enjoying a brisk hike through the forest. You round a bend and stop dead in your tracks - a large bear is on the trail ahead, staring directly at you. Your attention is entirely focused on this unexpected and potential threat. You remain unharmed, but you will remember this moment for years to come. The GANE (glutamate amplifies noradrenergic effects) model provides a compelling account of how arousal at the moment of experience leads to selective memory for prioritized information - for example, the bear. In the aftermath of emotional events, however, we often remember other details that seemed inconsequential at first, but were experienced in connection to the emotional event. For example, you might also remember seeing a fresh animal print in the mud earlier in your hike. These memories are adaptive; you do not want to wander unprepared into bear territory again. How we selectively remember information that occurred minutes to hours before an emotional experience is outside of the scope of the GANE model, but is well explained by a tag-and-capture model of memory consolidation.
Tag-and-capture refers to a model by which memory traces that are tagged during learning can benefit from periods of enhanced plasticity prior to or after learning, by capturing the plasticityrelated products (PRPs) necessary for long-term consolidation (Redondo \& Morris 2011; Viola et al. 2014). A key feature of this model is that weakly encoded memories stand the most to gain from this form of modulation, in that they are insufficient to drive long-term consolidation on their own. Moreover, the tag and capture phases need not occur simultaneously but can be separated by minutes to hours as long as they affect the same neural targets. Although tag-and-capture models were initially applied to electrophysiological studies of long-term potentiation (Frey \& Morris 1997; 1998), it has since been shown that salient or arousing experiences, such as novelty exposure, can rescue weak memories (Moncada \& Viola 2007; Wang et al. 2010) that overlap with the salient event (Ballarini et al. 2009).

A critical distinction between the GANE and tag-and-capture models is the time scales on which they are expected to operate. The GANE model proposes simultaneous engagement of noradrenaline and glutamate systems to enhance memory. Because this model necessitates coincidence detection across these neurotransmitter systems, the time frame by which arousal can facilitate learning is limited to the duration of salient memoranda (i.e., the source of glutamate). In contrast, studies of behavioral tagging indicate that a salient experience can strengthen weak memories encoded up to 2 hours prior to the salient experience. In fact, behavioral tagging of some forms of hippocampus-dependent learning is more effective if the salient experience is introduced about an hour before or after weak learning, compared with close in time (on the order of minutes) to the weakly learned event (de Carvalho Myskiw et al. 2014). In this way, tag-andcapture models are better able to explain extended effects of salience, including arousal, on memory for relatively remote events.

The GANE and tag-and-capture models also make different predictions with respect to which kinds of information are selected for consolidation. The GANE model proposes a combination of enhanced plasticity for prioritized information and suppression of non-prioritized information, with priority determined by intrinsic salience or attentional selection at the time of learning. In contrast, tag-and-capture models rely on the presence of an encoding tag at the site of enhanced plasticity. This allows the tag-and-capture mechanism to prioritize information after the time of learning, depending on which information turns out to be most relevant to the salient event (Ballarini et al. 2009; Dunsmoor et al. 2015). Thus, whereas the GANE model predicts memory improvements for prioritized information that coincides with an arousing event, tag-and-capture models predict memory improvements for information that acquires significance by virtue of its overlap with a separate arousing event. It is worth noting that both sets of mechanisms can, in theory, be deployed at any site of plasticity, offering flexibility in terms of which learning systems can benefit from arousal.

The relative temporal flexibility of tag-and-capture results from mechanisms that are distinct from GANE, including dopaminergic neuromodulation (Redondo \& Morris 2011). Critically, the dopaminergic system has properties that allow it to support consolidation at extended time scales. First, dopamine release in response to arousal is characterized by tonic, as opposed to phasic, activation (Grace et al. 2007), such that a single arousing event could result in prolonged increases in dopaminergic tone and facilitated learning (Shohamy \& Adcock 2010). Second, dopamine acts on relatively late stages of memory consolidation, allowing for salient events and encoding to be disparate in time. That is, dopamine affects protein synthesis-dependent long-term potentiation-a process necessary for consolidation - as opposed to memory encoding via early long-term potentiation (Lisman et al. 2011). Because dopamine-mediated synthesis of PRPs can occur independently from encoding, it may be particularly relevant for the consolidation of weakly encoded events, relative to strongly encoded events that are able to initiate PRP synthesis on their own through mechanisms 
like those described in GANE. It is worth noting that there is some evidence that, like dopaminergic responses, noradrenergic responses can be long-lasting (McIntyre et al. 2002) and involved in tag-and-capture effects (Moncada et al. 2011). However, additional research is needed to understand to what extent these neurotransmitter systems support memory consolidation at different time scales and for different kinds of information.

To conclude, the GANE and tag-and-capture models are complementary in that they can explain a range of memory phenomena occurring at and around the time of an arousing event. The GANE model makes novel predictions for what separates what we remember from what we forget, whereas tag-and-capture models are better suited to explaining why we often remember information from a window of minutes to hours around an emotionally salient event. Thus, the brain's ability to select information for consolidation into long-term memory is not determined only by the cognitive and neurobiological mechanisms operating at the moment of encoding. Rather, an adaptive memory system prioritizes the salient, but also allows the seemingly mundane to take on significance following new meaningful experiences.

\section{Importance of amygdala noradrenergic activity and large-scale neural networks in regulating emotional arousal effects on perception and memory ${ }^{1}$}

\author{
doi:10.1017/S0140525X15001934, e222
}

\author{
Benno Roozendaal, ${ }^{a, b}$ Laura Luyten, ${ }^{\text {a,b,c }}$ Lycia D. de Voogd, ${ }^{\text {a,b }}$ \\ and Erno J. Hermans ${ }^{a, b}$ \\ ${ }^{a}$ Department of Cognitive Neuroscience, Radboud University Medical Center, \\ 6500 HB Nijmegen, The Netherlands; ${ }^{\mathrm{b}}$ Donders Institute for Brain, Cognition \\ and Behaviour, Radboud University Nijmegen, 6525 EN Nijmegen, The \\ Netherlands; ' Center for the Psychology of Learning and Experimental \\ Psychopathology, KU Leuven, 3000 Leuven, Belgium. \\ benno.roozendaal@radboudumc.nl \\ laura.luyten@ppw.kuleuven.be linda.devoogd@donders.ru.nl \\ erno.hermans@donders.ru.nl \\ http://www.ru.nl/donders/research/theme-3-plasticity/
}

Abstract: Mather and colleagues postulate that norepinephrine promotes selective processing of emotionally salient information through local "hotspots" where norepinephrine release interacts with glutamatergic activity. However, findings in rodents and humans indicate that norepinephrine is ineffective in modulating mnemonic processes in the absence of a functional amygdala. We therefore argue that emphasis should shift toward modulatory effects of amygdala-driven changes at the network level.

Emotional arousal enhances memory of currently relevant - that is, salient - information, whereas it can impair memory of irrelevant information (Bennion et al. 2013; Mather \& Sunderland 2011). Mather et al. formulate the interesting hypothesis that when norepinephrine (NE) release coincides with high glutamatergic activity within an activated brain region or neuronal ensemble, NE release is increased further, resulting in locally enhanced neuronal activity and better memory. In contrast, when NE release does not coincide with high glutamate levels, NE suppresses neuronal activity, resulting in memory impairment. Although their model incorporates interactions at the systems level, it places strong emphasis on local processes, creating $\mathrm{NE}$ "hotspots." Here, we argue that such primarily local effects underestimate the importance of modulatory influences of the amygdala on encoding and consolidation of information throughout the network and that, without a functioning amygdala, such NE hotspots might be unable to affect local mnemonic processes.

According to the widely accepted "amygdala modulation hypothesis," basolateral amygdala (BLA) activity enhances memory of emotionally arousing experiences by influencing neural plasticity mechanisms in target regions elsewhere (McGaugh 2002). In rodents, pharmacologically enhancing or reducing noradrenergic activity within the BLA, that is, mimicking different arousal conditions, is sufficient to alter training-associated neural plasticity in distal brain regions (Beldjoud et al. 2015; McIntyre et al. 2005) and to determine whether neural representations in these other areas are being strengthened (Roozendaal \& McGaugh 2011). Recent evidence suggests that such BLA interactions with other brain regions not only modulate the strength of memory, but also are significantly involved in regulating memory precision (Ghosh \& Chattarji 2015), and that NE activity in particular may be the driving force behind improved accuracy (Barsegyan et al. 2014). Human neuroimaging research corroborates these findings by showing that amygdala activity during encoding of emotionally arousing stimuli predicts enhancement of hippocampus-dependent memory (Canli et al. 2000; Hamann et al. 1999). ß-Adrenoceptor blockade during encoding abolishes the emotional memory enhancement effect (Cahill et al. 1994) and suppresses memory-related amygdala activity (Strange \& Dolan 2004). Amygdala-hippocampal connectivity, furthermore, is stronger for emotionally arousing than for neutral stimuli (Dolcos et al. 2004), and the dominant directionality of this connectivity is indeed from amygdala toward hippocampus (Fastenrath et al. 2014).

Critically, amygdala-NE interactions selectively enhance memory for emotionally arousing as compared with neutral stimuli (e.g., Cahill et al. 1994). Mather et al. posit that the amygdala modulation hypothesis explains this selectivity in terms of a trade-off in which resources are shifted toward the emotional stimuli. However, recent findings indicate that there may be more to it than a simple trade-off. For example, Lovitz and Thompson (2015) report that intra-BLA infusion of the $\beta$-adrenoceptor agonist clenbuterol induces a long-term increase in excitability of hippocampal neurons when administered after emotionally arousing inhibitory avoidance training, but that clenbuterol decreases hippocampal excitability in non-trained control animals. These findings strongly support the idea that the impairing effects of amygdala-NE interactions on memory of nonsalient/non-arousing information involve an active process that is dependent on the amygdala.

Converging human evidence for this notion comes from patients with damage to the amygdala. For example, patients with Urbach-Wiethe disease (UWD), who exhibit selective calcifications in the BLA (Terburg et al. 2012), fail to show emotional enhancement of episodic memory (Cahill et al. 1995). Furthermore, studies in patients with other forms of amygdala pathology revealed a deficit in upregulating processing of emotional stimuli in higher-order visual cortices (Vuilleumier et al. 2004), as well as an impairment in increasing encoding-related hippocampal activity for emotional items (Richardson et al. 2004). Critically, UWD patients also exhibit enhanced memory for neutral information encountered in close temporal proximity to emotionally arousing stimuli (i.e., diminishing the impairment for such information observed in healthy controls [Strange et al. 2003]). One could argue that such findings remain consistent with an interpretation in terms of local hotspots of NE activity if amygdala damage would lead to a general impairment of NE signaling. However, UWD patients, although they fail to acquire conditioned responses, appear to exhibit normal arousal responses, as evidenced by normal skin conductance and startle responses to unconditioned stimuli (Bechara et al. 1995; Klumpers et al. 2015). Thus, findings from amygdala-lesioned patients agree with work in animals in suggesting that because of BLA damage, NE is ineffective in modulating local memory processes elsewhere in the brain.

Other studies have indicated that stress-related hormones such as glucocorticoids also contribute to selective enhancement of emotional memories. For example, in humans, elevating stress hormone levels after learning generally leads to consolidation benefits for emotionally arousing as compared with neutral information 
(Abercrombie et al. 2006; Kuhlmann \& Wolf 2006). Work in rodents has shown that NE activity within the amygdala also crucially determines the modulatory effects of stress hormones on neural plasticity and memory in distal brain regions (Roozendaal et al. 1999). The synthetic glucocorticoid dexamethasone, given immediately after inhibitory avoidance training, enhances longterm memory of this training in rats with an intact BLA, but dexamethasone impairs inhibitory avoidance memory if noradrenergic activity in the BLA is blocked with a $\beta$-adrenoceptor antagonist (Quirarte et al. 1997). Thus, these findings again support a critical role for BLA noradrenergic activity in determining enhancements or impairments of information storage in other brain regions.

In conclusion, local hotspots of NE activity at sites where mnemonic operations take place alone cannot explain the selectivity afforded by amygdala-driven modulatory processes. This observation, of course, raises the question of what mechanism underlies these distant modulatory effects. Important clues have come from functional connectivity studies in humans, showing that modulated regions are part of distinct large-scale neural systems, such as the "salience" and "default mode" networks (Hermans et al. 2011; 2014). Novel technologies for electrophysiological recordings and optogenetics in rodents are beginning to make it possible to study such networks in unprecedented spatiotemporal detail. We predict that these developments will ultimately lead to the conclusion that selective processing of arousing material results primarily from amygdala-driven changes in network properties of large-scale neural systems, rather than NE-induced local hotspots of activity.

\section{ACKNOWLEDGMENTS}

We acknowledge the financial support from Radboud University Nijmegen Topfund to Benno Roozendaal and the KU Leuven Center for Excellence Grant PF/10/005, and from Research FoundationFlanders (FWO) Research Grant 1504614N to Laura Luyten.

\section{NOTE}

1. BR and LL contributed equally to the preparation of this commentary.

\section{Locus coeruleus reports changes in environmental contingencies}

\author{
doi:10.1017/S0140525X15001946, e223
}

\section{Susan J. Sara}

Centre for Interdisciplinary Research in Biology, CNRS UMR 7142, Collège de France, 75005 Paris, France.

susan.sara@college-de-france.fr

Abstract: The GANE (glutamate amplifies noradrenergic effects) model proposed by Mather et al. attempts to explain how norepinephrine enhances processing in highly activated brain regions. Careful perusal of the sparse data available from recording studies in animals reveals that noradrenergic neurons are excited mainly by any change in the environment-a salient, novel, or unexpected sensory stimulus or a change in behavioral contingencies. This begets the "network reset hypothesis" supporting the notion that norepinephrine promotes rapid cognitive and behavioral adaption

The functional significance of neuronal activity in a particular brain region or population of neurons is found in the environmental stimuli or cognitive context that drive (or inhibit) activity in those neurons. Thus, we know from electrophysiological exploration that the function of primary visual cortex is to respond to light, that of auditory cortex, to sound, and so forth. Thalamic nuclei have likewise been delineated in terms of function. The role of prefrontal cortex in working memory was hypothesized by lesion studies, but clearly demonstrated by recording neuronal activity in monkeys performing working memory tasks (Fuster
1991; Goldman-Rakic 1990). Likewise, single unit recording in rats performing spatial navigation tasks established the fundamental role of the hippocampus in spatial cognition (O'Keefe \& Dostrovsky 1971). One of the principal functions of neurons of the ventral tegmental area is to report reward prediction error, based on recordings from this region in monkeys performing operant tasks. Thus, to gain a full understanding of the functional role of the locus coeruleus-noradrenergic system (LC-NE), it is important to carefully consider what drives this small population of neurons. Until recent biotechnological developments, the only way to achieve this was through recording activity of LC neurons in unanesthetized animals in carefully controlled behavioral situations. Given the inaccessible pontine location and very small size of this nucleus, the task has proved to be challenging and the resultant literature quite sparse. Nevertheless, there are some studies that provide insight that goes beyond LC-NE mediation of arousal and response to salient, stressful, or novel stimuli, as summarized by Mather et al. in section 4.1. Furthermore, recent advances in functional magnetic resonance imaging resolution have allowed imaging of this nucleus in humans performing complex cognitive tasks. These studies are now corroborating a role for LC in cognitive flexibility and behavioral adaptation, already demonstrated by electrophysiological studies in animals.

The earliest recordings of activity of LC in behaving rats established its role in vigilance and its responses to salient environmental stimuli in all modalities (Aston-Jones \& Bloom 1981). Subsequent experiments in rats and monkeys showed that LC neurons display remarkable plasticity as a function of environmental contingencies. Sensory responses habituate after just a few repetitions, even when initially robust, when no behavioral adaptation is required (HervéMinvielle \& Sara 1995). In a hole board environment, encounter with a novel object elicits a robust phasic burst of LC neurons that persists for only one or two subsequent investigations of the object (Vankov et al. 1995). Differential conditioning studies have shown that LC cells are exquisitely sensitive to stimulus-reward contingencies, showing task-related responses at the very earliest stages. At the beginning of training, both conditioned stimuli and primary reward elicit phasic responses in LC neurons. After just a few trials, response to reward disappears and response to the stimulus predicting reward $\left(\mathrm{CS}_{+}\right)$increases, whereas response to the neutral stimulus (CS-) decreases. These discriminative conditioned responses in LC appear many trials before any behavioral expression of learning and before task-related responses emerge in the prefrontal cortex. They are not maintained during overtraining, but when contingencies change abruptly, as during extinction or reversal training, phasic LC responses are immediately reinstated, tens of trials before behavioral expression of learning (Bouret \& Sara 2004; Sara \& Segal 1991). Similar phenomena have been reported for behaviorally contingent LC activity in monkeys (Aston-Jones et al. 1997; Rajkowski et al. 2004).

These relatively sparse data collected from behaving rats and monkeys over a span of 25 years led us to hypothesize that NE released in the cortex in response to a salient event or to a sudden change in environmental contingencies may act to facilitate or promote a rapid change in cortical state, "reset" the active network, and drive an adaptive behavioral response (Bouret \& Sara 2005; Sara \& Bouret 2012). We have provided some preliminary evidence for a "reset" action of NE, revealed by spike-triggered wave form averages of gamma filtered local field potential. Gamma band synchronization (GBS) has functional roles in diverse cognitive processes, including attention, stimulus processing, decision making, and response timing (Bosman et al. 2014). We found a strong temporal relation between GBS and spontaneous LC bursts. In fact, LC spiking interrupts the gamma wave for about $200 \mathrm{~ms}$, with the recovered GBS having increased power (Poe and Sara 2014; Sara 2015, Fig. 3).

Recent functional magnetic resonance imaging studies in humans have lent strong support to a prediction of Corbetta et al. (2008) that there should be a strong functional relation between the ventral frontoparietal network, involved in 
Commentary/Mather et al.: Norepinephrine ignites local hotspots of neuronal excitation

"resetting" attention, and LC neuronal activity, given the striking similarity between the environmental contingencies driving them. In a recent study requiring subjects to continually modify their behavior as a function of unpredictable changes in stimulusresponse contingencies, switches elicited activation in a frontoparietal network that has been implicated in task switching and error awareness, in concert with activation of the brainstem LC (von der Gablentz et al. 2015). Several other similar studies in humans have confirmed that LC is co-activated with frontal regions or has increased functional connectivity with them in cognitively demanding tasks requiring rapid shifts in allocation of attention (see Sara 2015 for a review). This rapidly growing literature has strongly supported the earlier electrophysiological data from animal studies leading to the network reset hypothesis of the functional role of the LC-NE system.

The GANE model proposed by Mather et al. is complementary to the "reset" hypothesis. The model provides a basis for understanding how NE biases perception and promotes synaptic plasticity and memory formation in select target regions that are engaged by current contingencies. However, the efficacy of glutamate recruitment of the LC-NE system to enhance processing of significant stimuli will depend, at least in part, on action potentials in LC neurons. These neurons are driven by environmental imperatives for a rapid cognitive shift and behavioral adaptation. Thus, we conclude that the overarching function of the LC-NE system is to promote rapid change in ongoing network activity (Bouret \& Sara 2005). GANE provides a testable model of how subsequent release of $\mathrm{NE}$ can provide selective enhancement of the reorganized network.

\section{Bidirectional synaptic plasticity can explain bidirectional retrograde effects of emotion on memory}

\author{
doi:10.1017/S0140525X15001958, e224
}

\section{Bryan A. Strange ${ }^{a, b}$ and Ana Galarza-Vallejo ${ }^{a}$}

a Laboratory for Clinical Neuroscience, Centro de Tecnología Biomédica, Universidad Politecnica de Madrid, Pozuelo de Alarcón, Madrid 28223, Spain; ${ }^{b}$ Department of Neuroimaging, Reina Sofia Centre for Alzheimer's Research, Madrid 28031, Spain. bryan.strange@upm.es www.thestrangelab.org

Abstract: Emotional events can either impair or enhance memory for immediately preceding items. The GANE model explains this bidirectional effect as a glutamate "priority" signal that modulates noradrenaline release depending on arousal state. We argue for an alternative explanation: that priority itself evokes phasic noradrenaline release. Thus, contrasting E-1 memory effects are explained by a mechanism based on the Bienenstock-Cooper-Munro theory.

An emotional stimulus is typically well remembered but also influences memory for temporally adjacent events. In humans, we reported an emotion-induced retrograde impairment of memory in the context of shallow encoding of word lists containing an occasional emotional (E) noun (Strange et al. 2003). This retrograde disruption for "E-1" nouns appears to be mediated by the amygdala via a noradrenergic (NE) mechanism, as it is blocked by the $\beta$-adrenergic antagonist propranolol (Strange et al. 2003). However, subsequent studies have indicated that, if the encoding task requires that attentional weight be given to each E-1 stimulus, these stimuli show memory enhancement (Anderson et al. 2006; Knight \& Mather 2009). Mather et al. propose that for tasks involving attention to E-1 items, this "priority" signal is mediated by glutamate. According to their model, in a state of arousal, this elevated glutamate level associated with highly active neural representations stimulates greater NE release, leading to enhanced encoding of E-1 stimuli.

We propose that the opposing retrograde effects of emotion on memory can be explained by an alternative, simpler model. We propose that "priority" itself is coded by phasic NE release in the brain. Attending to task-relevant cues has been found to increase activity in the locus coeruleus (LC) in non-human primates (Aston-Jones et al. 1994). Thus, high-“priority” E-1 encoding is likely to be associated with moderate levels of LC activity (Figure 1A, bottom). Given that enhanced memory for emotional items is blocked by propranolol, we assume that these emotional items provoke LC activity (Figure 1A, bottom). Because of the aversive nature of the $\mathrm{E}$ stimuli, this LC activity is likely to be greater than that evoked by task-relevant E-1 items. By contrast, in the case of low-"priority" E-1 encoding, E-1 items trigger minimal LC activity (Figure 1A, top).

The bidirectional effects of emotion on memory for E-1 items can then be explained by a non-linear relationship between LC activity to E-1 items and memory encoding. According to the Bienenstock-Cooper-Munro model (Bienenstock et al. 1982),
A

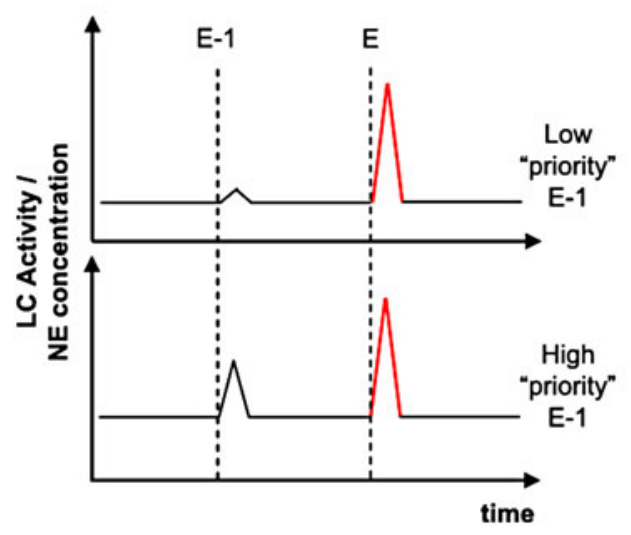

B

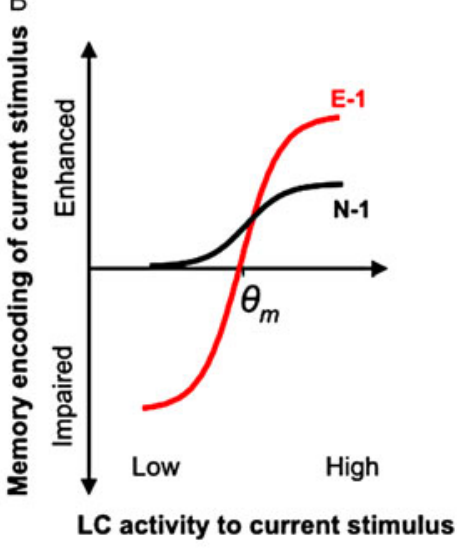

Figure 1 (Strange \& Galarza-Vallejo). Alternative model for bidirectional retrograde effects of emotion on memory. (A) LC responses are illustrated schematically to the presentation of two pairs of E-1 and E items (stimulus onset is indicated by vertical dashed lines). If the E-1 item is of high priority (i.e., the encoding task requires attention to this item), the LC response is higher than that to a low-priority E-1 item. Subsequent presentation of the E item triggers greater LC activity. (B) Hypothesized likelihood of encoding the current neutral stimulus, as a function of LC activity to that stimulus, depending on whether the subsequent stimulus is emotional (red curve) or neutral (black). If the subsequent stimulus is emotional (i.e., triggers large NE release), low E-1 LC activity is more likely to lead to subsequent forgetting of the E-1 stimulus. $\theta_{\mathrm{m}}=$ modification threshold. 
when the postsynaptic cell is weakly depolarized by other inputs, active synapses undergo long-term depression (LTD) as opposed to long-term potentiation (LTP) (Dudek \& Bear 1992; Abraham $\&$ Tate 1997). The modification threshold, $\theta_{\mathrm{m}}$, is the measure of postsynaptic activity that determines the direction of synaptic-efficacy change. In this scheme, if postsynaptic activity is below $\theta_{\mathrm{m}}$, but above baseline, synaptic efficacies are weakened. Conversely, if postsynaptic activity exceeds $\theta_{\mathrm{m}}$, synapses are strengthened. In Figure 1B, we apply this model to E-1 memory encoding (red curve). For low-priority E-1 items, postsynaptic activity is below $\theta_{\mathrm{m}}$ at the time of LC responses to the $\mathrm{E}$ noun, leading to a weakening of the efficacy of synapses that were engaged during the immediately preceding E-1 encoding (Diamond et al. 2004). For high-priority E-1 items, postsynaptic activity is already relatively high (above $\theta_{\mathrm{m}}$ ) when the $\mathrm{E}$ stimulus is presented, yielding memory enhancement (red curve in Figure 1B). Note that the bidirectionality of this proposed effect is dependent on the presentation of $\mathrm{E}$ items. The black curve in Figure $1 \mathrm{~B}$ represents memory for a stimulus that precedes a neutral (N) item (i.e., an N-1 stimulus) plotted as a function of the LC activity to this stimulus. Obviously, if, for any reason, this "N-1" stimulus evokes LC activity, its memory will be enhanced, but not to the level of enhanced E-1 memory. Importantly, N-1 memory will not be impaired even if it is low priority.

Thus, applying a model of the bidirectional nature of synaptic plasticity (Bienenstock et al. 1982) that has been validated in the context of $\mathrm{NE}$ stimulation (Hu et al. 2007; Kemp \& Manahan-Vaughan 2008) can fully explain retrograde memory effects of emotion in a parsimonious way. The change in synaptic efficacy most likely occurs within a limited brain circuit involving amygdala and hippocampus (Strange \& Dolan 2004), with NE input from the LC. It will be interesting to test whether contexts proposed to modulate $\theta_{\mathrm{m}}$, such as stress (Kim \& Yoon 1998), will alter the direction of memory modulation for $\mathrm{E}-1$ items for a given encoding task. Interestingly, blocking $\beta$-adrenergic receptors with propranolol does not abolish the emotion-induced retrograde amnesia for low-priority E-1 stimuli, but actually enhances memory for these E-1 items (Strange et al. 2003). It is tempting to speculate that propranolol decreases $\theta_{\mathrm{m}}$ (i.e., shifts the red curve in Figure $1 b$ to the left), such that low levels of LC activity to low-priority E-1 nouns become associated with better memory.

\section{Emotionally arousing context modulates the ERP correlates of neutral picture processing: An ERP test of the GANE model}

doi:10.1017/S0140525X1500196X, e225

\section{Deborah Talmi and Gemma E. Barnacle \\ School of Psychological Sciences, University of Manchester, University of Manchester, M139PL Manchester, United Kingdom. deborah.talmi@manchester.ac.uk gemmaebarnacle@gmail.com www.psych-sci.manchester.ac.uk/staff/talmi/}

\begin{abstract}
The time scale of the effects of emotional arousal on neutral information processing is crucial for the predictions of the glutamate amplifies noradrenergic effects (GANE) model. GANE suggests that when emotional and neutral stimuli are presented in a sequence, neutral information processing will change. We review the literature on eventrelated potentials, including our own data set, to test this prediction.
\end{abstract}

The time scale of the facilitating versus impairing effects of emotional arousal on the processing of neutral information is an open question for the glutamate amplifies noradrenergic effects (GANE) model (sect. 7.2). The authors assert that "an emotionally salient word that impairs perception of a subsequent target word flashed in the same location 50 or $100 \mathrm{~ms}$ later can instead enhance perception of a target word flashed 1,000 ms later" (sect. 2.1). The specific time scales are likely to vary across experimental setups: for example, depending on the complexity of stimuli and the intensity of the arousal. The problem is that if the impact of arousal is not temporally bound, priority can be used to explain experimental effects in either direction, namely, both the impairing and the facilitating effects of arousal. Here we discuss how EEG data can provide crucial temporal dynamic information that can disambiguate GANE's predictions-evidence that Mather et al. did not consider.

According to GANE, arousing stimuli capture resources during their processing. Once their own processing is completed, the arousal they induce also facilitates the processing of subsequent stimuli. To test GANE we need to know in advance the duration of emotional stimulus processing. Previous work indicates that emotional pictures, a stimulus of choice in much of the human emotion-cognition literature, enhance a number of eventrelated potentials (ERPs). The most robust is the late positive potential (LPP). The LPP is thought to reflect attention allocation and maintenance of stimuli in working memory (Donchin \& Coles 1988). The amplitude of the LPP 400-700 ms after stimulus presentation is higher when stimuli are emotional (Schupp et al. 2006), reflecting the additional resources allocated to such stimuli, in line with GANE. Emotion also enhances other components, including the positive slow wave, where amplitudes are higher up to $6 \mathrm{~s}$ post-stimulus. We can therefore conclude that the processing of neutral information presented within $6 \mathrm{~s}$ of emotional pictures may be attenuated.

Only with this temporal information can we put GANE to the test. We do so by comparing the ERPs associated with processing neutral stimuli presented on their own (blocked neutral condition) with those presented alongside emotional stimuli (mixed condition). Not only is the context more arousing in the mixed condition (Long et al. 2015), but also emotional stimuli increase arousal locally. When the interstimulus interval (ISI) is long, emotional and neutral stimuli are unlikely to compete for processing resources, and GANE predicts that the higher global arousal in the mixed condition should enhance neutral information processing. In contrast, Pastor et al. (2008) used an ISI of $12 \mathrm{~s}$ and observed reduced LPPs for neutral stimuli in the mixed compared with the blocked condition. It is, however, possible that those emotional pictures were still being processed when the subsequent picture was displayed after $12 \mathrm{~s}$. When the ISI is short, competition should be pronounced, so GANE predicts that the processing of neutral information should be impaired. In contrast, Schupp et al. (2012) used an interval of $3 \mathrm{~s}$ and observed a null effect of context (blocked/mixed). It is, however, possible that the effect of emotion on resource allocation in that study was short-lived, for example, because of the orienting task. If emotional stimuli no longer attract attention when subsequent neutral stimuli are presented, the null effect is incompatible with GANE's predictions.

In our experiment (Barnacle et al. 2015), 22 healthy adults viewed 16 lists of 14 pictures: 4 neutral, 4 emotional, and 8 mixed lists $(50 \%$ emotional pictures). All pictures depicted people; emotional and neutral picture sets were equally semantically related, but the emotional pictures were more negative and arousing. Each picture was presented for $2 \mathrm{~s}$ with a jittered ISI of $4 \pm 0.5 \mathrm{~s}$. Participants were asked to encode these pictures for a free-recall memory test, which followed each study list after a 60-second distractor task. EEG was recorded during encoding with a BioSemi Active Two (BioSemi, Amsterdam) using 64 electrodes conforming to the 10-20 system and preprocessed with SPM (www.fil.ion.ucl.ac.uk). Data were filtered between 0.1 and $25 \mathrm{~Hz}$, downsampled to $125 \mathrm{~Hz}$, and epoched between -200 and 4,000 ms. Eye-blink artifact was removed using an algorithm implemented in SPM. A threshold of $120 \mu \mathrm{V}$ was used for trial rejection followed by robust averaging.

Following Schupp et al. (2012), we extracted LPP and slowwave component amplitude data, averaging across centroparietal electrodes $(\mathrm{Cz}, \mathrm{CPz}, \mathrm{Pz}, \mathrm{C} 1, \mathrm{C} 2, \mathrm{P} 1, \mathrm{P} 2, \mathrm{CP} 1, \mathrm{CP} 2)$ in three time windows: $400-700 \mathrm{~ms}, 1,000-2,000 \mathrm{~ms}$, and 2,000-3,000 
Commentary/Mather et al.: Norepinephrine ignites local hotspots of neuronal excitation

LPP

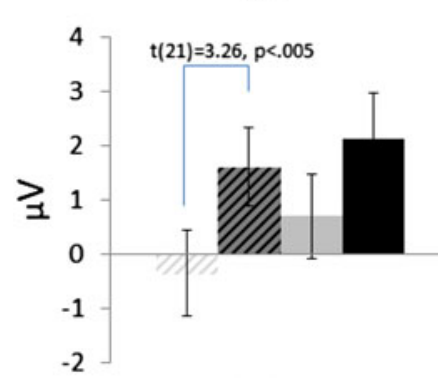

$400-700 \mathrm{~ms}$

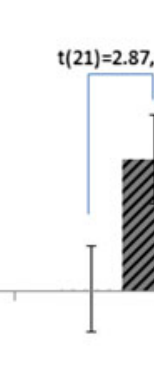

Slow Wave

Neutral Mixed

$\checkmark$ Emotion Mixed

$1-2 s$

$2-3 s$

Figure 1 (Talmi \& Barnacle). Average ERP amplitude in time windows corresponding to the Late Positive Potential and to the early portion of the positive slow wave. Emotion modulation lasts up to 2,000 ms poststimulus.

ms poststimulus. We compared emotional and neutral picture processing in the mixed condition at each window to ascertain the duration of the effects of arousal, using three one-tailed paired-sample $t$-tests $(p<0.017$ controlled for multiple comparisons). Emotion modulated ERPs in the 400-700 ms and 1,000$2,000 \mathrm{~s}$ windows, but not later (Figure 1). We then compared neutral picture processing in the mixed and blocked conditions at both these windows with two two-tailed t-tests $(p<0.025)$. The LPP for neutral pictures was slightly, but not significantly attenuated in the mixed, compared with the blocked, condition.

Our data indicate that the duration of the effect of arousal is key for testing GANE in novel experimental setups. EEG data allowed us to determine how long emotional pictures attracted extra processing resources. Here the modulation lasted up to $2 \mathrm{~s}$ from stimulus onset, evident in the modulation of the early portion of the LPP and positive slow wave, but not later. This pattern suggests that arousing stimuli are no longer in competition for resources when neutral pictures are presented in the same sequence 3.5-4.5 $\mathrm{s}$ after the onset of the arousing pictures, and their prioritized processing should not detract from the resources allocated to neutral stimuli. On the contrary, because of the increased global arousal in the mixed condition, GANE predicts that neutral picture processing should be enhanced. In fact, our data provided evidence that the processing of neutral information is attenuated in that situation.

The three ERP data sets we reviewed appear to contradict GANE's predictions. The mature electrophysiology literature on the effect of emotion on perception, attention, and memory (e.g. Hajcak et al. 2010; Schupp et al. 2006) can provide crucial data for GANE until data on the times scales of norepinephrine-glutamate interactions are available.

\section{What BANE can offer GANE: Individual differences in function of hotspot mechanisms}

\author{
doi:10.1017/S0140525X15001971, e226
}

Rebecca M. Todd, ${ }^{a}$ Mana R. Ehlers, ${ }^{a}$ and Adam K. Anderson ${ }^{b}$ ${ }^{a}$ Department of Psychology, University of British Columbia, Vancouver, BC, Canada, V6T 1Z4; ${ }^{\mathrm{b}}$ College of Human Ecology Department of Human Development, Cornell University, Ithaca, NY 14853.

becket.todd@psych.ubc.carmanaehlers@psych.ubc.ca

aka47@cornell.edu

http://mclab.psych.ubc.ca/

http://www.human.cornell.edu/bio.cfm?netid=aka47

Abstract: In this commentary we focus on individual differences in proposed mechanisms underlying arousal-based enhancement of prioritized stimuli. We discuss the potential of genotyping studies for examining effects of noradrenergic processes on stimulus prioritization in humans and stress the importance of potential individual differences in the activity of specific receptor subtypes in hotspot processes proposed by the GANE model.

We believe that the GANE (glutamate amplifies noradrenergic effects) model makes a novel and vital contribution to understanding emotional modulation of attention and memory - specifically via its detailed description of the influence of glutamate on noradrenergic processes mediating the impact of emotional arousal on the fate of neutral items. However, Mather et al. do not address potential individual differences in such mechanisms, which may be linked to mood disorders and addiction.

In our BANE (biased attention via norepinephrine) model, we described effects of the locus coeruleus-norepinephrine system in enhancing attention and memory for stimuli that are salient because of associations with arousal (Markovic et al. 2014). However, a key focus of the BANE model was individual differences in prioritization of affectively salient stimuli (Markovic et al. 2014; Todd et al. 2012). This emphasis on individual differences has been based in part on observations of human carriers of a common deletion variant in the $A D R A 2 b$ gene, which codes for noradrenergic $\alpha_{2 B}$-autoreceptors (Small et al. 2001). The GANE model makes a valuable contribution in extending beyond the BANE model to incorporate the role of glutamatergic activity in enhancing effects of arousal on processing stimuli that are already high priority. However, the authors are somewhat dismissive of studies examining polymorphisms in genes coding for noradrenergic receptors and, specifically, of the notion that findings concerning the role of ADRA2 $b$ can be discussed in relation to the GANE model's hotspot mechanisms. They do so based partly on evidence that $\alpha_{2 \mathrm{~B}}$-receptors are unlikely to play an important role in GANE hotspots because the inhibitory role of $\alpha_{2 \mathrm{~B}}$-receptors is not as well established as for $\alpha_{2 \mathrm{~A}}$-receptors and because $\alpha_{2 \mathrm{~B}}$-receptors are poorly expressed in key regions mediating affective salience. We argue that the study of genetic influences on affective prioritization of salient stimuli can provide data relevant to some of the GANE model's claims, and that evidence against an inhibitory role of $\alpha_{2 B}$-receptors in key brain regions is not entirely straightforward.

First, we argue that genotyping studies have value, in general, for understanding mechanisms of stimulus prioritization because, along with pharmacologic manipulations (e.g., De Martino et al. 2008; Strange et al. 2003), they are among the few vehicles for examining effects of inhibitory versus excitatory noradrenergic processes in humans. Because the specificity of ligands for receptor subtypes is limited (Jasper et al. 1998), genotyping studies can help specify the role of each subtype in patterns of brain activation and behavior. Of course, we acknowledge that it is important to use other methods, such as positron emission tomography and examination of mRNA activity, to help confirm the role of specific ADRA2 $a$ and ADRA2 $b$ polymorphisms in $\alpha_{2}$ activity.

Second, it is important to consider potential individual differences in the activity of specific receptor subtypes in proposed hotspot 
processes and what the behavioral consequences might be. Genetic differences influencing such receptor function are one source of such differences, and can provide a valuable window into how GANE mechanisms can vary normally and go awry. For example, common variants in genes coding for both ADRA2 $b$ and ADRA2a receptors have been associated with neural and behavioral indices of enhanced attention and memory for affectively salient stimuli that characterize affective disorders as well as cognitive biases associated with addictive behaviors (de Quervain et al. 2007; Havranek et al. 2015; Todd et al. 2013). Using genotyping to infer the role of each receptor subtype on such endophenotypes can help elucidate how patterns of inhibitory/excitatory activity proposed by GANE may contribute to variation in healthy populations and in psychopathology.

Studies of the ADRA2 $b$ deletion variant can serve precisely that function. Convergent evidence is highly consistent with the view that ADRA2b deletion carriers have reduced inhibitory autoreceptor function. In vivo, consequences of carrying the $A D R A 2 b$ deletion variant (found in $\sim 50 \%$ of the populations we have studied) are similar to those of the $\alpha_{2}$-antagonist yohimbine (de Quervain et al. 2007). This claim is supported by the reliability and robustness of effects of enhanced emotional biases in attention and memory, increased amygdala and ventromedial prefrontal activation for arousing stimuli, and differences in amygdala gray matter volume associated with carrying the deletion variant (de Quervain et al. 2007; Ehlers et al. 2015; Rasch et al. 2009; Todd et al. 2013; 2014; 2015). According to the GANE model, affectively salient stimuli are one category of prioritized stimulus whose encoding is enhanced by arousal. Here, the enhanced affective prioritization we have observed in deletion carriers could lead to intensified positive feedback loops at hotspots, although possibly only when stimuli are prioritized because of their pre-existing associations with arousal. Further, because outside of the lab there are likely to be a range of motivationally relevant goals, behavior of deletion carriers may be driven by affective or visual salience over more "top-down" goals relative to non-carriers.

Finally, with regard to the authors' claims that it is $\alpha_{2 \mathrm{~B}}$-autoreceptors that carry the full burden of inhibitory function in the brain, we suggest that the picture is somewhat more complicated. There is evidence that, in addition to its pre-synaptic inhibitory function, $\alpha_{2 \mathrm{~A}}$ is the most commonly observed postsynaptic receptor in the prefrontal cortex (Arnsten et al. 1996; U'Prichard et al. 1979). Indeed, some evidence suggests that increased post-synaptic $\alpha_{2 \mathrm{~A}}$ activity in the PFC may be associated with enhanced rather than reduced noradrenergic transmission (Ramos et al. 2006). Moreover, brain regions mediating heightened emotional sensitivity in deletion carriers show relatively high levels of ADRA2b expression (Hawrylycz et al. 2012: Allen Human Brain Atlas: http://human.brain-map.org). Animal research points further toward the importance of $\alpha_{2 \mathrm{~B}}$-receptors in emotional processing (Moriceau \& Sullivan 2004). This challenges the notion of a straightforward role for $\alpha_{2 \mathrm{~A}}$-receptors as the only mediators of inhibitory activity suggested by the GANE model.

In summary, although we acknowledge that effects of the deletion variant may be mediated by factors other than proposed GANE hotspot mechanisms, the growing body of research on polymorphisms influencing both a $\alpha_{2 A^{-}}$and $\alpha_{2 B}$-receptors poses both questions and challenges for the GANE model.

\section{Cognitive control, dynamic salience, and the imperative toward computational accounts of neuromodulatory function}

\section{doi:10.1017/S0140525X15001983, e227}

\section{Christopher Michael Warren, Peter Richard Murphy, and Sander Nieuwenhuis}

Institute of Psychology and Leiden Institute for Brain and Cognition, Leiden University, 2311 EZ, Leiden, The Netherlands. c.m.warren@fsw.leidenuniv.nl

p.murphy@fsw.leidenuniv.nl_snieuwenhuis@fsw.leidenuniv.nl http://www.temporalattentionlab.com

Abstract: We draw attention to studies indicating that phasic arousal increases interference effects in tasks necessitating the recruitment of cognitive control. We suggest that arousal-biased competition models such as GANE (glutamate amplifies noradrenergic effects) may be able to explain these findings by taking into account dynamic, within-trial changes in the relative salience of task-relevant and task-irrelevant features. However, testing this hypothesis requires a computational model.

Mather and colleagues' GANE (glutamate amplifies noradrenergic effects) model offers a compelling and neurobiologically principled account of how phasic arousal and the associated release of norepinephrine (NE) benefit perception and memory of salient, high-priority information at the expense of lower-priority information. Here, we draw attention to a directly relevant line of research that the authors do not address, concerning the effect of phasic arousal in tasks that necessitate the online recruitment of cognitive control. In such task contexts, exogenously salient but misleading aspects of the stimulus must be ignored in favor of less conspicuous but task-relevant information. Thus, there is competition for representation between stimulus features that have bottom-up salience and features that are assigned priority via the top-down deployment of attention.

Participants are typically able to perform such tasks at a high level of accuracy, indicating that the task-relevant stimulus features generally win this competition. Yet, contrary to straightforward predictions of "winner-take-more/loser-take-less" models of arousal-biased competition like the GANE model, it has repeatedly been shown that a phasic arousal boost in such contexts leads to increased interference effects (i.e., decreased accuracy and a relative slowing of response times when the task-irrelevant feature and/or its associated response are incongruent with the task-relevant information) (Böckler et al. 2011; Callejas et al. 2005; 2004; Correa et al. 2010; Fan et al. 2002; 2009; Fischer et al. 2010; Klein \& Ivanoff 2011; MacLeod et al. 2010; Weinbach \& Henik 2012; 2014).

Close examination of the behavior on cognitive control tasks has yielded an important insight that helps to reconcile winner-takemore models with this arousal-driven increase in interference effects. Specifically, empirical conditional accuracy functions (Gratton et al. 1988; Hommel 1994) and computational analyses (Cohen et al. 1992; Nieuwenhuis \& de Klein 2013; White et al. 2011) indicate that the relative salience of task-irrelevant and task-relevant stimulus features on such tasks changes rapidly over the course of a single trial: Early in a trial, the bottom-up salience of the task-irrelevant information dominates the competition, but as cognitive control develops, the top-down salience of task-relevant information increases and usually wins the competition. In such a scenario, instantiation of a winner-take-more regime via a phasic increase in arousal would initially serve to enhance the early dominance of the task-irrelevant information and, depending on the duration of the phasic arousal response, may make it more difficult for the task-relevant information to eventually win out. Thus, by drawing on the idea of dynamic, time-variant salience, GANE may in principle be able to explain the well-documented interactions between arousal and cognitive control.

A clear implication of time-varying salience is that the predicted neural and behavioral outcomes of the winner-take-more/losertake-less effects of glutamate-NE interactions will be critically dependent on the time scale over which these interactions occur. We believe that this point poses a key challenge for the GANE model, which in its current form lacks the level of analysis required to generate formal predictions of this nature: that is, it lacks a computational level of analysis that explicitly links neurobiology to behavior.In the domain of cognitive control tasks, without model simulations it is unclear whether the transient NE-mediated enhancement in processing occurs early in the trial when 
the task-irrelevant information dominates processing, later in the trial when cognitive control has prioritized task-relevant information, or both. Similar model simulations incorporating the timing of NE-mediated processing enhancements are also necessary to confirm whether GANE can account for the differential pattern of arousal effects on memory for stimuli occurring before and after arousing events (Sakaki et al. 2014a).

In principle, GANE may be implemented in the form of a detailed biophysical model (e.g., Eckhoff et al. 2009; Wang 2002) that simulates the cascade of neurochemical events at the "NE hotspots" described by Mather and colleagues. This component of the model would need to interact with other biophysically realized components that sustain associated cognitive functions (decision-making, cognitive control, memory) and generate task behavior, and the model predictions will depend on the interactions between these component processes and their relative timing. However, the fidelity of biophysical detail in such a model will likely trade off with its ability to provide a unified explanation of the vast array of arousalrelated behavioral effects reviewed in Mather et al.

An alternative, perhaps more feasible approach would be for Mather and colleagues to adopt a simplified computational model of NE function that captures the essential impact of NEglutamate interactions on task performance, in a form that is computationally tractable and can therefore be leveraged to generate predictions based on GANE principles in a wide variety of behavioral contexts. Indeed, a class of connectionist models of NE function already exists that appears well suited to such a pursuit. In these models, NE modulation is implemented as a multiplicative change in the input-to-output function of a task processing unit otherwise known as a change in "gain" - and produces the critical winner-take-more/loser-take-less effects that GANE attempts to account for (e.g., Eldar et al. 2013; Servan-Schreiber et al. 1990). These models have been successfully adapted to explain neuromodulatory effects on perception and memory in a wide variety of task contexts, including those that require the online recruitment of cognitive control. Moreover, the model components governing NE modulation can be implemented at multiple levels of abstraction, from single model parameters that are global and time invariant (Eldar et al. 2013; Servan-Schreiber et al. 1990), to fine-grained subnetworks that operate on biophysically realistic principles and afford precise control over timing (Gilzenrat et al. 2002; Nieuwenhuis et al. 2005b; Usher et al. 1999). In our view, whether the research question of interest pertains to arousal/cognitive control interactions or otherwise, this type of broadly applicable, computationally tractable modeling framework will be necessary to generate and test precise predictions of the GANE model in the future.

\section{Author’s Response}

\section{GANEing traction: The broad applicability of NE hotspots to diverse cognitive and arousal phenomena}

\author{
doi:10.1017/S0140525X16000017, e228
}

\author{
Mara Mather, ${ }^{\mathrm{a}}$ David Clewett, ${ }^{\mathrm{b}}$ Michiko Sakaki, ${ }^{\mathrm{c}}$ and \\ Carolyn W. Harley ${ }^{d}$ \\ ${ }^{a}$ Davis School of Gerontology, University of Southern California, Los Angeles, \\ CA 90089-0191; ${ }^{\mathrm{b}}$ Neuroscience Graduate Program, University of Southern \\ California, Los Angeles, CA 90089-2520; ' School of Psychology and Clinical \\ Language Sciences, University of Reading, Reading RGX 7BE, \\ United Kingdom; and ${ }^{\mathrm{d}}$ Professor Emeritus, Memorial University of \\ Newfoundland, St. John's, NL A1C 5S7, Canada.
}

mara.mather@usc.edu

matherlab.com

clewett@usc.edu

http://dornsifecms.usc.edu/david-clewett-neuroscience/

m.sakaki@ reading.ac.uk

http://www.reading.ac.uk/psychology/about/staff/m-sakaki.aspx

charley@play.psych.mun.ca

https://www.mun.ca/psychology/bio/harley.php

Abstract: The GANE (glutamate amplifies noradrenergic effects) model proposes that local glutamate-norepinephrine interactions enable "winner-take-more" effects in perception and memory under arousal. A diverse range of commentaries addressed both the nature of this "hotspot" feedback mechanism and its implications in a variety of psychological domains, inspiring exciting avenues for future research.

We proposed the glutamate amplifies noradrenergic effects (GANE) model to answer the following question: What are the brain mechanisms that allow arousal to simultaneously enhance processing of salient or high-priority stimuli and impair processing of inconspicuous or low-priority stimuli? In our model, the local level of glutamatergic neurotransmission signifies the priority of an activated representation. In the cortex, when glutamate spillover from activated synapses activates $N$-methyl- $D$-aspartate (NMDA) receptors on nearby varicosities of locus coeruleus (LC) neurons that are being depolarized by LC action potentials, this leads to more local release of norepinephrine (NE), which further amplifies glutamate release and enhancement of the information the highly excited neurons are representing. Elsewhere, lower glutamate levels fail to ignite hotspots and undergo greater suppression via NE-induced inhibition. We proposed that, in addition to enhancing activation of prioritized representations, the NE-glutamate hotspot effects selectively recruit metabolic resources, enhance neuronal oscillations, and trigger synaptic plasticity processes that enhance long-term memory of prioritized information.

Across the commentaries discussing GANE's relevance to cognitive and neural processes, several important themes emerged (see Table R1). Generally, the responses can be grouped as having one of two foci (with some exceptions): behavioral and cognitive aspects of the arousal by priority interaction relevant to GANE or the NE hotspot mechanism itself.

To predict which information will be selectively enhanced or impaired by arousal, it is important to focus on the two key factors necessary to ignite a hotspot: (1) an arousing-inducing stimulus that can stimulate LC activity (NE), and (2) a stimulus that has high priority (glutamate). As outlined in Table R1, several of the commentaries elaborated on these two factors, as well as on other issues and themes. We discuss the issues raised in the commentaries here in our response, starting with the topic of arousal.

\section{R1. Arousal}

A number of the commentaries raise questions regarding arousal.

\section{R1.1. Nature of arousal}

In our view, the LC-NE system is not the only brain system involved in a generalized arousal response (see Pfaff 2006 
Table R1. General topics raised in commentaries.

What elicits LC activity?

Forms of priority

How does GANE operate in relation to specific aspects of brain function?

Spatial extent of hotspots

What are the adaptive functions of the neural effects of NE?

Relevance of GANE in various domains

Factors that should be addressed

Alternatives to GANE
Higher levels of arousal associated with uncertainty may help new salient information gain priority via hotspot mechanisms, whereas lower levels of arousal may protect existing strong predictions from distracting information under conditions of high certainty (Nassar, Bruckner, \& Eppinger [Nassar et al.]).

Prediction errors may trigger a phasic NE response that facilitates the selective updating of predictions in the prioritized manner outlined by GANE (Ferreira-Santos).

Competition elicits arousal, which leads to an increase in theta and gamma oscillations that select and stabilize "winning" representations (Phaf).

Negative stimuli might evoke more arousal than positive stimuli (Kaspar).

Fluently processed stimuli yield a stronger signal (or are more salient), and so GANE can explain how arousal amplifies responses to these stimuli (Carbon \& Albrecht).

Commentators discussed dendritic integration (Larkum \& Phillips), relative timing of oscillatory patterns (Phaf), the role of the dentate gyrus in memory selection (Houghton), and genetic variations in the ADRA2B gene (Todd, Ehlers, \& Anderson [Todd et al.]).

Eldar, Cohen, \& Liv (Eldar et al.) recognize that in the GANE model, hotspots would be co-extant with distributed cortical representations, whereas Gaucher \& Edeline are expecting more spatially extensive loci. This difference in visualization highlights the need for tools to identify active hotspot elements. Immediate early genes may be useful in this regard.

GANE may be a general-purpose function that cuts across a variety of cognitive and

Salpehariatseffiggter (Hull)C to release NE cortically, which facilitates a "network reset" that promotes quick changes in cortical states and adaptive behavioral responses (Sara). Salient stimuli may predict threatening or significant stimuli (Bouret).

Stress. Endocrine signals, in particular cortisol, work in tandem with NE to promote long-term adaptive changes and memories (Hurlemann, Maier, \& Scheele

[Hurlemann et al.]).

Sleep and memory. Acetylcholine is likely to have hotspot properties different from those of NE, and so low NE and high acetylcholine during REM sleep may help explain lack of memory for dreams (Becchetti \& Amadeo).

Early development. The LC exhibits developmental changes during infancy and early development, and early life stress shapes glutamate and GABA responses in ways that should be considered in the GANE model (Geva).

Responses to sexual stimuli. Contrary to expectations of posture showing approach/ avoidance biases, people viewing either threatening or sexual stimuli exhibit a freezinglike reaction in which they are more immobile (Mouras).

Emotion regulation. Arousal levels should influence the ability to alter behavioral responses (Hull).

Appraisal theory. Stimuli that are relevant for individuals' goals, needs, and values induce strong arousal and amygdala activity (Montagrin \& Sander).

Commentators pointed out that GANE requires further development to specify timing (Talmi \& Barnacle; Navarra \& Waterhouse; Warren, Murphy, \& Nieuwenhuis [Warren et al.]), address different effects in prefrontal cortex (Abdallah, Averill, Krystal, Southwick, \& Arnsten (Abdallah et al.]), examine context and individual differences in determining salience (Huntsinger \& Storbeck), address role of $\boldsymbol{\alpha}_{\mathbf{1}^{-}}$ receptors (Navarra \& Waterhouse), and address how cardiac afferents influence how LC modulates cortical activity (Critchley \& Garfinkel).

Priority is coded by phasic NE release and so there is no need for glutamate to signal priority (Strange \& Galarza-Vallejo; see response in sect. R4.1)

The amygdala is necessary for NE to enhance selective processing and memory consolidation of arousing stimuli (Roozendaal, Luyten, de Voogd, \& Hermans

[Roozendaal et al.]; see response in sect. R4.2)

The tag-and-capture model is better able than GANE hotspot mechanisms to explain the effects of arousal on memories for events that occurred minutes to hours before the arousing event (Ritchey, Murty, \& Dunsmoor [Ritchey et al.]; see response in sect. R5).

Countering the target article's argument that a "network reset" model could not account for enhanced memory for well-attended items seen before an arousing event, Bouret argued that such enhanced memories could be accounted for by network reset if the qualitative nature of the representation changed (see response in sect. R6). 
for a review of arousal pathways in the brain), but its activation is a common theme that runs through all different modes of arousal. For instance, NE inputs to cells in the ventromedial hypothalamus are critical in initiating sexual arousal (Pfaff 2006; of relevance for Mouras' commentary), whereas noradrenergic input to the amygdala is critical in enhancing memory for emotionally arousing stimuli (see Roosendaal et al.'s commentary and sect. R4.2 on the role of the amygdala).

What is arousal? At the most basic level, we have the contrast between sleep and wakefulness. NE is low during most sleep states (see Becchetti \& Amadeo). Then, during wakefulness, physical activity increases NE (Carter et al. 2010). But in addition to these broad-scale changes, the arousal system is also exquisitely sensitive and can adapt rapidly to small changes in the environment or internal goals.

These arousal responses can be detected by measuring pupil dilation. NE system activity increases pupil dilation, as NE released by the LC inhibits pupil constriction (Koss et al. 1984; Wilhelm 2008). Pupils are constricted during sleep, compared with wakefulness (Yoss et al. 1970). During wakefulness, aerobic exercise (Ishigaki et al. 1991) or muscular exertion (Nielsen \& Mather 2015; Nielsen et al. 2015) increases pupil dilation. Arousal induced by stimuli or tasks also increases pupil dilation. For example, emotionally arousing scenes (Bradley et al. 2015), sexually arousing stimuli (Bradley et al. 2015), surprise, uncertainty, loud noises, and cognitive effort all increase pupil dilation. Subjective arousal ratings given for emotional images correlate with pupil diameter during viewing (Bradley et al. 2008). These consistencies across different elicitors of arousal suggest they share some underlying mechanisms to modulate cognitive and brain processing. Eldar et al. review a recent line of work in which they used pupil dilation as a marker of $\mathrm{NE}$ activity and found that indices of high NE function are associated with increased selectivity in learning, perception, and memory, consistent with their neural network models in which $\mathrm{NE}$ was modeled as global increase in gain. GANE complements and extends this approach by providing hypotheses about how NE implements neural gain.

We agree with Mouras and Kaspar regarding the relevance of sexual arousal and internal sources of arousal (such as from one's thoughts). Our point of view is that these different types and sources of arousal can be accommodated by the GANE model, as evidence suggests that LC activation is a common theme for all of them.

\section{R1.2. How the heartbeat influences $L C$ activity}

The LC is influenced not only by external stimuli and an individual's own thoughts, but also by interoceptive signals. For example, distension of the bladder or colon increases LC activity (Elam et al. 1986), whereas an increase in blood pressure decreases LC activity (Elam et al. 1984). LC neurons also exhibit cardiac periodicity. For example, in cats, LC neurons are most likely to fire 80-180 ms after the peak of the cardiac R-wave (during diastole) and least likely to fire $40 \mathrm{~ms}$ before to $60 \mathrm{~ms}$ after the R-wave (during systole) (Morilak et al. 1986).

Critchley \& Garfinkel have shown that stimuli detection and memory encoding differ during the systole (contraction) and diastole phases of the heartbeat. During systole, participants are better able to detect fear (but not neutral) faces in an attentional blink paradigm and rate them as more intense (Garfinkel et al. 2014). When words are the T2 stimuli in an attentional blink paradigm, later memory for the words depends on both the confidence with which the words were originally detected and at what heartbeat phase the words were detected (Garfinkel et al. 2013). Words detected with high confidence during systole have a memory advantage, whereas words detected with low confidence during systole have a memory disadvantage. Therefore, during systole, highly salient stimuli such as fear faces and clearly detected target words get a boost in processing or later consolidation. But why would this GANE-like pattern occur during systole when the LC neurons are less likely to fire? This surprising aspect of the findings suggests the possibility that LC activity and salient glutamatergic representations may interact best when they are offset slightly in time.

Critchley \& Garfinkel argue that the GANE notion that LC-NE activity amplifies salience is not sufficient to account for their findings because their cardiac cycle effects sometimes appear to be driven by fear rather than arousal more generally. However, as illustrated in their figure, there was not a significant difference between fear and disgust or happy faces, and the disgust and happy faces showed trends toward enhancement where neutral faces showed a trend toward impairment at diastole. Fear faces are often more salient than happy or disgust faces (Anderson et al. 2003; Mather \& Knight 2006); therefore, we think more work is needed before a specific-emotion account must be invoked in place of a salience mechanism such as that provided by GANE.

\section{R1.3. How arousal may amplify the salience of negative stimuli}

Kaspar makes the case that negative stimuli may be more likely than positive stimuli to ignite neuronal hotspots because of the evolutionary pressure not to miss potential threats. One challenge is how to test this hypothesis, as negative stimuli, on average, induce more arousal than positive stimuli (Grühn \& Scheibe 2008), and so any differences in processing or memory between negative and positive stimuli could be due to different levels of arousal when processing them, rather than to different levels of priority. To try to address this question, we recently ran a study in which we induced arousal independently by having participants squeeze a ball in their hand as hard as they could before they viewed emotional pictures and examined how the resulting increases in arousal influenced memory for the pictures (Nielsen et al. 2015). We were interested in hormonal effects, and all participants were younger female women. Consistent with Kaspar's predictions, we found that handgripinduced arousal enhanced memory for the negative, but not the positive pictures. This effect was most pronounced for women with low estrogen and progesterone levels at the time of testing.

Kaspar also suggested that because of declines in the LC-NE system, negative stimuli lose their arousing potential as people age. However, the evidence suggests that the older adults' positivity effect is not due to a lack of bottom- 
Response/Mather et al.: Norepinephrine ignites local hotspots of neuronal excitation

up salience for negative stimuli. Like younger adults, older adults look first at arousing stimuli regardless of their valence (Knight et al. 2007) and notice arousing or threatening stimuli more quickly than other types of stimuli (Leclerc \& Kensinger 2008; Mather \& Knight 2006). Bottom-up affective salience should play less of a role in influencing processing for low-arousal pictures, and indeed, the positivity effect appears to be stronger among valenced stimuli low rather than high in arousal (Kensinger 2008). In addition, we found that arousal induced by handgrip selectively benefited memory encoding of negative pictures (compared with positive or neutral pictures) in older women not taking hormone supplements, as well as in younger women with low estrogen and progesterone levels (Nielsen et al., in preparation). The evidence thus suggests that arousing negative pictures have similar bottom-up salience for older and younger adults.

\section{R1.4. Relation between arousal and appraisal theory}

On the basis of appraisal theory, Montagrin \& Sander raise a question about how arousal and priority interact. They argue that arousal and goal relevance are not independent and stimuli that are relevant for individuals' goals, needs, and values induce strong arousal and amygdala activity. We agree with them: Given that the LC exhibits phasic activity in response to goal-relevant stimuli (Aston-Jones \& Cohen 2005; Aston-Jones et al. 1999), it seems possible that goal-relevant stimuli become arousing. However, the appraisal theory approach they discuss does not detail the neural mechanisms by which arousal induced by goal-relevant stimuli helps people memorize (Montagrin et al. 2013) and prioritize attention to those stimuli (Pool et al. 2015). In contrast, our GANE model can explain their findings of enhanced processing of goalrelevant stimuli: once the amygdala and/or prefrontal regions detect goal-relevant stimuli and recruit the LC (see Sara \& Bouret 2012 for discussion of amygdala and prefrontal inputs to LC), NE hotspots will be generated in circuits transmitting goal-relevant information and, in turn, hotspots will enhance memory and perception for those stimuli. Therefore, GANE does not contradict the appraisal model, but instead extends it.

\section{R1.5. Arousal and emotion regulation}

Hull argues that the role of arousal in GANE is relevant for understanding impairments in emotion regulation. In particular, when stuck on a particular representation associated with negative emotions, decreases in arousal may be necessary to allow for less emotionally disturbing representations to be prioritized. Although not addressed in Hull's commentary, a related point is the relevance of GANE for disorders such as post-traumatic stress disorder (PTSD), in which intrusive thoughts are a problem. A particular disturbing thought or memory may induce arousal, which, in turn, enhances attention to and memory reconsolidation of that particular representation. On the basis of GANE, beta blockers during initial encoding or retrieval of the memory should attenuate the immediate strength of its activation and its long-term synaptic strength. Consistent with this are some observational findings suggesting that beta blockers may help prevent intrusive thoughts or PTSD (Krauseneck et al. 2010; Lindgren et al. 2013), although random assignment has yielded some null effects (Stein et al. 2007).

\section{R2. Priority}

Other commentaries focused on physiological and psychological aspects of priority, a key factor in GANE.

\section{R2.1. Perspectives on physiological mechanisms of priority}

Larkum \& Phillips describe a novel physiological mechanism by which contextual information modulates pyramidal cell activity. Neocortical pyramidal cell bodies have an apical trunk that ascends to a dendritic branching pattern called an apical tuft, which resides in a different cortical layer than the cell body and the basal dendrites around it. The long distance of the apical tuft from the cell body sets it up to serve a modulatory role in driving cell activity (Phillips 2015). Apical amplification could, for example, provide top-down priority selection of a quiet bottom-up auditory input to cortical output circuits. In their figure, Larkum \& Phillips illustrate the interaction between GANE and apical amplification priority, providing an experimentally testable physiological model. Houghton argues that, computationally, the mossy cell hilar circuit in hippocampus would set priority for hippocampal processing and suggests heavy hilar NE innervation is consistent with GANE amplification of that mechanism. Becchetti \& Amadeo make the interesting point that conscious (and, thus, prioritized) oneiric processing occurs during rapid eye movement (REM) sleep, likely supported by high acetylcholine modulation. But with active suppression of LC-NE during REM, there is little or no memory of those priority events, also consistent with GANE.

\section{R2.2. Possible relation between fluency and priority}

Carbon \& Albrecht point out that fluency (i.e., processing information more easily) is an important factor determining stimulus priority. Greater fluency can arise because of perceptual salience (e.g., reading a word printed in a clear, high-contrast font more quickly than a blurry word) or because of prior knowledge or experience (e.g., reading a familiar word more easily than an unfamiliar word). Previous findings had suggested that people feel more positively about stimuli that they process more fluently (e.g., Winkielman \& Cacioppo 2001). In a recent study, Albrecht and Carbon (2014) presented affective pictures that were either preceded (507 ms earlier) by that same image or by a different image shown for only $7 \mathrm{~ms}$ and asked participants to rate the valence of the pictures. There was no main effect of valence, but, instead, an amplification effect, with highly positive pictures rated more positively when they had been primed and highly negative pictures rated more negatively when they had been primed. Insofar as fluently processed stimuli yield higher glutamatergic activity than less fluently processed stimuli (something that seems plausible but remains to be tested) and that the emotional stimuli elicited arousal, their findings that valence judgments of emotional stimuli are amplified by fluency fit with GANE. 
Response/Mather et al.: Norepinephrine ignites local hotspots of neuronal excitation

\section{R3. Predictive utility of GANE}

Huntsinger \& Storbeck and Talmi \& Barnacle argued that GANE does not provide clear predictions concerning whether the presentation of emotionally arousing stimuli would enhance or impair cognitive processing of stimuli that appear nearby in time or space. Huntsinger \& Storbeck state that GANE can provide post hoc explanations about the effects of emotional stimuli in a range of situations, but they question GANE's predictive utility. Talmi \& Barnacle also argue that because we don't know exactly how long emotional stimuli dominate competition for representation, we can explain either the enhanced or impaired effects of emotional stimuli on nearby neutral stimuli by GANE.

We agree with them that it is hard to determine priority when comparing emotional with neutral stimuli. As discussed in our target article, emotional stimuli tend to have higher priority than neutral stimuli because of their goal relevance, bottom-up salience, and emotional salience. Thus, in the hypothetical experiment Huntsinger \& Storbeck mention, where emotional stimuli are presented as distractors with task-relevant neutral stimuli, emotional distractors can have higher priority than neutral goal-relevant stimuli. This could especially be the case when the topdown control mechanisms are not strong enough to establish the goal relevance of neutral stimuli (see Warren et al.).

Talmi \& Barnacle suggest that one can get around the issue of the different salience between emotional and neutral stimuli by having a long interval between emotional and subsequent neutral stimuli. But it is not clear that having a long interval would increase the priority of neutral stimuli as high as that of emotional stimuli. In addition, because high arousal can impair top-down prioritization (Arnsten 2011; Kuhbandner \& Zehetleitner 2011), top-down control mechanisms might fail to increase the priority of neutral stimuli presented after emotional stimuli. These considerations suggest that in their EEG study (Barnacle et al., in preparation), neutral stimuli intermixed with emotional stimuli still had lower priority than neutral stimuli presented in a neutral list, which led to the impaired processing of neutral stimuli in the intermixed condition as predicted by GANE. Furthermore, having a long interval has the disadvantage that the effects of phasic arousal and NE release might not last long enough to yield modulatory effects (see Section 9 in our target article).

In summary, it is difficult to test GANE in experimental settings where researchers simply include emotionally arousing stimuli and neutral stimuli without a clear manipulation of priority. In our view, to test GANE, it is important to manipulate the priority of neutral stimuli, independently from arousal (Lee et al. 2014b; Sakaki et al. 2014a; Sutherland \& Mather 2012). One way to achieve this in the context of Barnacle et al. (in preparation) would be to have high-priority neutral images and low-priority neutral images in the mixed list condition. Similar changes can be made in the bridge study mentioned by Huntsinger \& Storbeck (Dutton \& Aron 1974); GANE predicts that arousal induced by the scary bridge will enhance memory for nearby high-priority stimuli (e.g., a woman seen on the bridge if the participant were asked to approach a woman and ask her something) while impairing memory for nearby low-priority stimuli (e.g., a man on the bridge who has no task relevance or particular interest). In summary, GANE can provide clear predictions as long as priority levels can be manipulated or assessed in the experiment.

\section{R4. Alternatives to GANE proposed in commentaries}

Several of the commentaries propose alternatives to GANE to explain the mechanisms by which arousing stimuli affect cognitive processing.

\section{R4.1. NE-only model}

Strange \& Galarza-Vallejo propose that the glutamate aspect of the model is not necessary; they describe a simpler model in which priority is coded by phasic NE release in the brain. They work through an example from research on the emotional oddball-1 (E-1) effect, in which emotional oddballs (words or pictures) impair memory for the immediately preceding item on the list if that item was low priority for the participant, but enhance it if that item was high priority (e.g., Sakaki et al. 2014a). A problem with their NE-only model is that it is not clear how phasic NE release can selectively "tag" the E-1 item and not other items. Perhaps in the simple setup they describe, in which one word or object appears at a time in the list, phasic NE release could mark activated neural networks via a temporal tagging process. However, they do not consider findings that when multiple items are shown simultaneously, whether and how much memory for them is enhanced or impaired by a subsequent emotional item depends on their priority. For example, in an experiment in which a scene was shown either alone or with an object superimposed (Fig. R1A), if the image was followed by an emotional sound, there was impaired memory for the scene later, but only if it had been made lower priority by being in the background (Fig. R1B) (Ponzio \& Mather 2014). Likewise, in another study in which participants saw four items at the same time that were then followed by a tone that was conditioned to predict either a shock (CS+) or no shock (CS-), having a subsequent arousing tone affected later memory for the simultaneously shown items differently depending on the relative priority of the items (Lee et al. 2015). The model Strange and Galarza-Vallejo propose does not explain how phasic LC activation could have different effects on items shown at the same time. In our view, this is the main contribution of GANE: by positing a mechanism for local cortical modulation of NE, it provides the only explanation to date of how arousal can have simultaneous differential effects on items based on their priority.

\section{R4.2. Amygdala-based model}

Roozendaal et al. argue that the amygdala is necessary for NE to enhance selective processing and memory consolidation of arousing stimuli. We agree that the amygdala plays a critical role, but argue below that its role in mediating the effects of NE is necessary only when the amygdala is the primary site of the neural representation in question. 
A

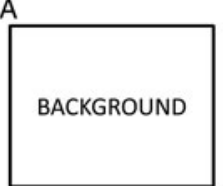

$2 \mathrm{sec}$

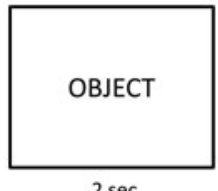

$2 \mathrm{sec}$

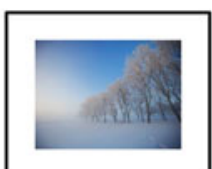

$2 \mathrm{sec}$

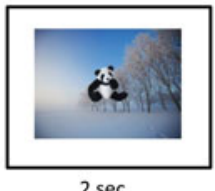

$2 \mathrm{sec}$

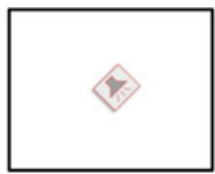

$4 \mathrm{sec}$

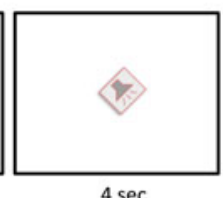

$4 \mathrm{sec}$

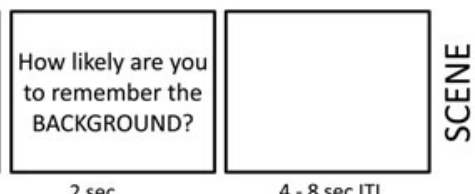

$4-8 \mathrm{sec} I \mathrm{TI}$

B

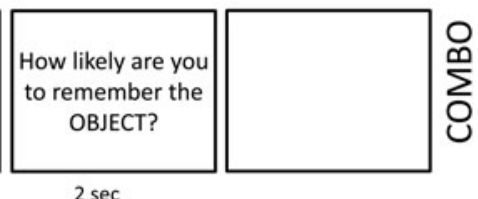
口Neutral Sound

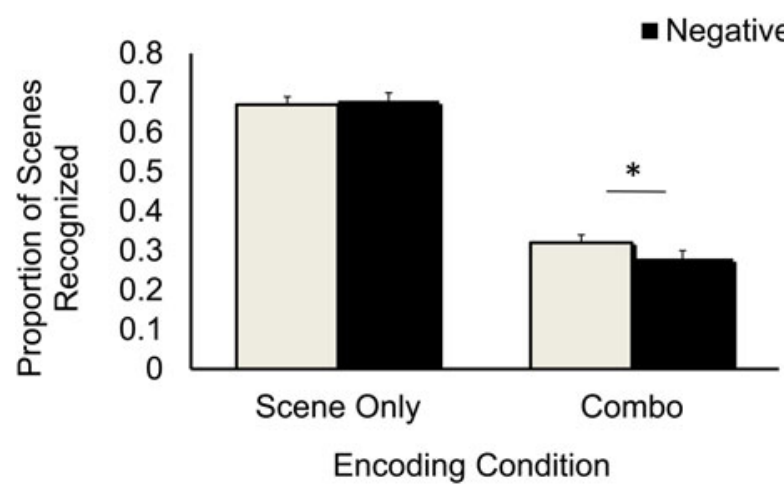

Fig. R1. (Mather et al.) (A) Arousing negative sounds were heard after seeing a background scene either alone or superimposed with a foreground object. (B) The arousing sounds impaired memory for the scene only when it was seen behind the object and, therefore, was somewhat suppressed by that competitor (Ponzio \& Mather 2014).

Data from individuals with amygdala lesions help reveal which types of representations depend on the amygdala and which types can be supported by other brain regions. Compared with controls, unilateral amygdala patients exhibited as much enhanced visual cortex activity when viewing emotionally salient images (Edmiston et al. 2013), as much of an advantage for detecting emotional targets (Piech et al. 2010), and as much emotional capture by emotional stimuli during an attentional blink task (Piech et al. 2011). Two individuals with selective bilateral amygdala lesions exhibited a significant advantage in recalling aversive (compared with neutral) words during an attentional blink task, and this advantage was as large as that seen for matched control participants (Bach et al. 2011). Someone with complete bilateral amygdala lesions who could not recognize fear from faces still showed normal rapid detection of those faces (Tsuchiya et al. 2009). Thus, the amygdala is not necessary for the initial selective attention and encoding advantages seen for emotionally arousing stimuli, suggesting that NE-glutamate hotspots in sensory brain regions can occur even in the absence of the amygdala.

In addition, highly salient sensory stimuli yield normal physiological responses in people missing amygdalae (e.g., Tranel \& Damasio 1989). For example, in studies of fear conditioning, individuals with amygdala lesions have normal skin conductance responses to aversive stimuli such as loud noises (Bechara et al. 1995; Klumpers et al. 2014). Likewise, three patients with bilateral amygdala lesions each had a panic attack when inhaling $35 \% \mathrm{CO}_{2}$
(Feinstein et al. 2013), indicating that amygdala lesion patients still experience fear in response to interoceptive alarming cues. These intact responses to interoceptive or external sensory stimuli contrast with the lack of fear shown by amygdala patients in response to experiences or visual stimuli (e.g., a haunted house or a live snake) that typically elicit fear because of their association with danger (Feinstein et al. 2011).

This pattern of findings suggests that the amygdala is essential for anticipatory physiological responses to stimuli that predict something aversive. This possibility is supported by fear conditioning studies with individuals with amygdala lesions (Bechara et al. 1995; Klumpers et al. 2014). These individuals lacked skin conductance responses to $\mathrm{CS}+$ cues that predicted loud noises, even though they acquired explicit knowledge about the CS+ contingency. In contrast, an individual with bilateral hippocampal lesions failed to acquire explicit knowledge about the contingency, but had skin conductance responses to the CS+ (Bechara et al. 1995). Therefore, amygdala lesions impair physiological responding to cues that predict threat, but do not impair explicit learning about these cues. Amygdala lesions also impair physiological responding to simulated monetary rewards and losses in the context of a gambling game (Bechara et al. 1999), indicating that the amygdala is necessary for an abstract stimulus predicting something positive or negative to yield a physiological affective response.

The findings that patients with amygdala lesions no longer have physiological responses to predictive cues 
despite having as much explicit knowledge of the contingencies as normal controls suggests that: (1) there are amygdala-based neural representations of associations between neutral cues and potential affectively relevant outcomes; and (2) these amygdala-based representations are necessary to trigger signals to sympathetic pathways to mount a physiological response, possibly in part via amygdala projections to the LC (Cedarbaum \& Aghajanian 1978).

Likewise, the finding that an individual with a hippocampal lesion lacked explicit knowledge of fear conditioning contingencies despite exhibiting a skin conductance response to the CS+ suggests that there also are amygdala-independent, hippocampus-based neural representations of associations between CSs and USs. However, in people with intact amygdalae and hippocampi, these separate representations in the two regions are likely to have close interactions, in part supported by a direct glutamatergic pathway from the basolateral amygdala to the CA1 region of the hippocampus (Rei et al. 2015).

Noradrenergic contributions to interactions between amygdala and hippocampus have been examined using one-trial learning to avoid a shock (McIntyre et al. 2005). In this paradigm, the $\beta$-adrenergic receptor agonist clenbuterol is infused into the basolateral complex of the amygdala shortly after a rat learns that moving from a brightly lit compartment of an alley through a door to a dark compartment is associated with a shock. The $\beta$-adrenergic stimulation of the amygdala increases Arc expression (indicating more synaptic changes occurred) in the hippocampus in the 45 min after the shock. Of particular relevance in this context, however, are findings that the increased Arc expression depends not only on greater NE activity in the amygdala itself, but also on arousal levels more generally (McReynolds et al. 2014). Specifically, whereas basolateral amygdala infusions of a $\beta$ agonist increased Arc protein levels for the inhibitory avoidance shock task, as seen in previous studies and also for a "high-arousal" version of an object recognition task, NE activity in the amygdala was not sufficient to increase Arc in the hippocampus when the object recognition task was not arousing. These findings suggest that glutamate-NE feedback loops in the amygdala can be intensified by within-amygdala local $\beta$-adrenergic activation (Fig. R2A). This hotspot activity increases glutamatergic signaling to the hippocampus (Fig. R2B) but does not directly increase NE levels in the hippocampus. However, the increased glutamatergic activity in the hippocampus can stimulate local release of NE via NMDA receptor activity at LC neuron varicosities if the LC is depolarized (Fig. R2C; see target article for more details on hotspot mechanisms). In summary, McReynolds and colleagues' data suggest that NE can influence hippocampal activity either indirectly via glutamatergic pathways from the amygdala or directly via local release from $\mathrm{LC}$ varicosities. More generally, we posit that $\mathrm{NE}$ action within the amygdala has important glutamatergic modulatory effects elsewhere in the brain (in particular in the hippocampus), but that the LC also modulates excitation and inhibition directly in these other brain regions via local release of NE. The critical experiments necessary to test this hypothesis have not been performed yet (see relevant proposed study in Table R2).

Roozendaal et al. also argue that "the impairing effects of amygdala-NE interactions on memory of non-salient/ non-arousing information involve an active process that is dependent on the amygdala." They make this case based on Lovitz and Thompson (2015), whom they interpret as showing that intra-basolateral amygdala infusion of a $\beta$ adrenergic agonist (clenbuterol) decreases hippocampal excitability in non-inhibitory avoidance-trained control animals. However, their interpretation appears to be incorrect, as in that study, there was no significant difference between vehicle and clenbutorol conditions in the untrained rats.

\section{R5. Role of NE hotspots in long-term memory formation}

Some commentaries raise questions concerning the role of NE hotspots in memory. First, Hurlemann et al. point out the importance of cortisol, in addition to NE and glutamate, in explaining the effects of arousal on memory. Combining neuroimaging with a psychopharmacological approach, Hurlemann et al. demonstrated that NE and glucocorticoids interact during processing of emotional stimuli (Hurlemann 2008; Kukolja et al. 2008; 2011). In particular, their work suggests that NE interacts with cortisol to enhance learning of emotional information within the amygdala-hippocampus network.

Acute stress and administration of glucocorticoids lead to enhanced glutamate release both in the amygdala (Reznikov et al. 2007) and in the hippocampus (Moghaddam et al. 1994) via mechanisms mediated by glucocorticoid receptors (GRs) and mineralocorticoid receptors (MRs) (for reviews, see Popoli et al. 2012; Sandi 2011). In the amygdala and hippocampus, interactions between glucocorticoids and NE have been observed, as well (for reviews Joëls et al. 2011; Krugers et al. 2012). These results suggest the interesting possibility that glucocorticoids help NE create hotspots in the amygdala-hippocampus circuit by enhancing glutamatergic activity. One question is whether the NE-cortisol interaction goes beyond the amygdala-hippocampus circuit. Although most previous research focuses on the effects of glucocorticoids either in the amygdala-hippocampus pathway or in the prefrontal cortex (PFC), glucocorticoids might also amplify NE hotspots in other cortical regions, given that GRs are widely expressed in brain (Morimoto et al. 1996). Furthermore, elevated cortisol and NE levels tend to impair goal-directed attentional processes in the PFC (Schwabe et al. 2012), which should enhance the impact of the bottom-up, salience-driven hotspots predominant in sensory brain regions.

Second, Ritchey et al. state that the tag-and-capture model is better able than GANE hotspot mechanisms to explain the effects of arousal on memories for events that occurred minutes to hours before the arousing event. For example, initially weak memories can be strengthened by a subsequent salient signal, such as a novelty or aversive event (Dunsmoor et al. 2015; Redondo \& Morris 2011). The tag-and-capture model explains these results by asserting that memory traces are tagged during initial learning, which allows for subsequent plasticity-related proteinmediated mechanisms to capture those tagged traces to create long-term memories. Ritchey et al. also argue that the effects of arousal on protein synthesis processes are mediated by dopaminergic neuromodulation. 

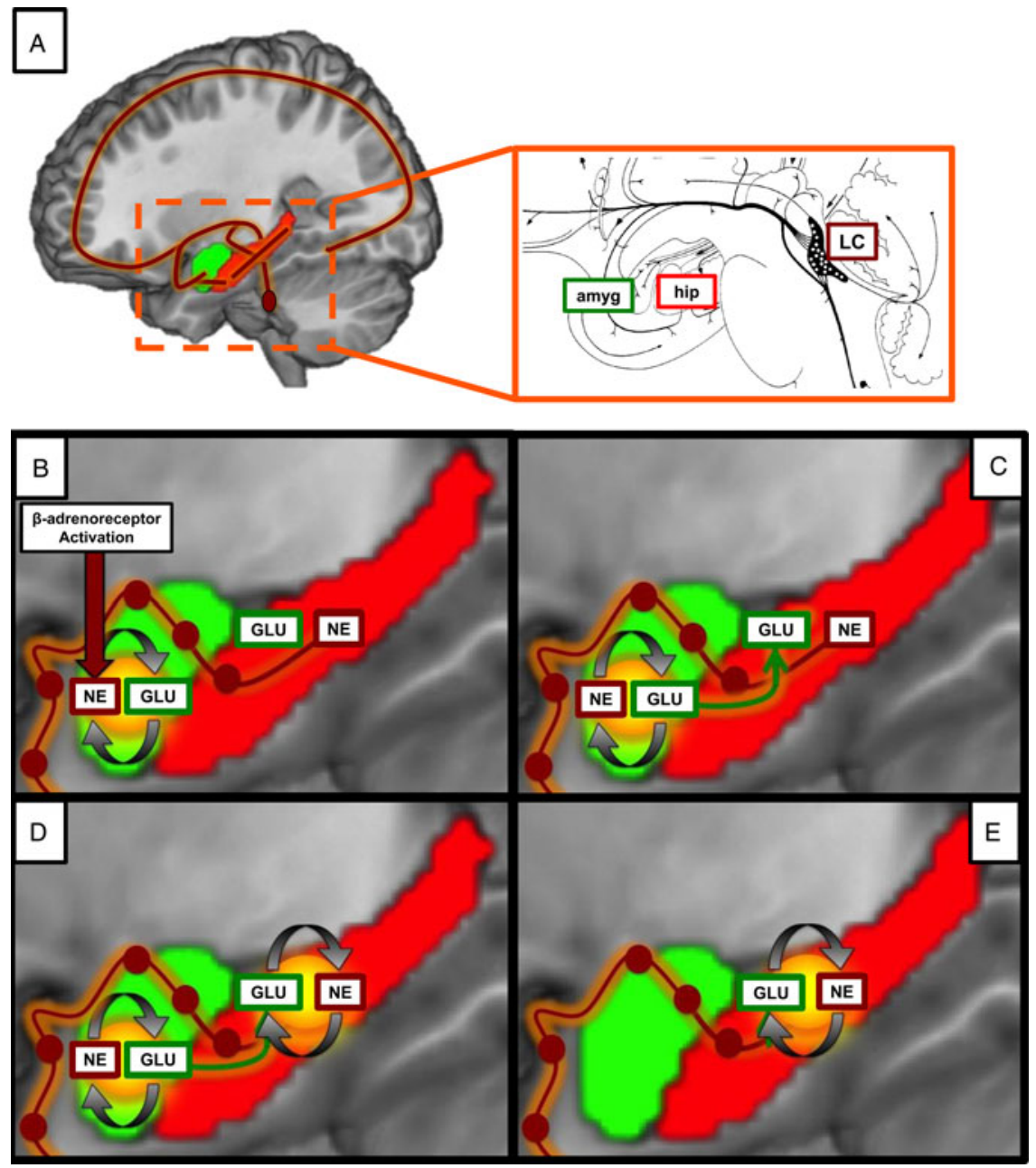

Fig. R2. (Mather et al.) Previous findings suggest that NE can influence hippocampal activity both directly from NE release from LC neurons and also via amygdala glutamatergic pathways. (A) LC neurons innervate both amygdala and hippocampus. (B) NE released during LC activation (or, in the case of McReynolds et al., 2014 discussed in the text, a $\beta$-agonist) interacts with activated local glutamatergic representations within the amygdala to create a hotspot of higher glutamatergic activity. (C) These glutamate-NE hotspots originating in the amygdala amplify hippocampal glutamatergic activity via glutamatergic pathways. Neurons originating in the amygdala connecting to the hippocampus do not release NE, and so amygdala activity will not directly affect NE levels in the hippocampus. (D) However, amygdala modulation of hippocampal glutamatergic activity can increase the probability of local hotspots developing in the hippocampus, because if the LC is depolarized, the amygdala-induced glutamatergic activation in the hippocampus stimulates local NE release and further amplifies glutamatergic activation via glutamate-NE hotspot mechanisms. (E) Even in the absence of amygdala modulatory activity, NE hotspots can develop in the hippocampus from excited glutamatergic activity interacting with NMDA receptors on local LC neuron varicosities to increase local release of NE, as demonstrated in hippocampal slice preparations (e.g., Lalies et al., 1988; Nelson et al., 1980; Pittaluga \& Raiteri, 1990; Vezzani et al., 1987; Wang et al., 1992). Note: Inset in (A) is reprinted with permission from Marien et al. (2004), p. 41, part of Figure 2.

Although in our target article we focused mainly on the immediate effects of NE hotspots, we believe that evidence indicates a role for these hotspots in tag-and-capture scenarios. $\beta$-Adrenergic receptor activity stimulates protein synthesis and gene expression alterations associated with long-term potentiation maintenance (Maity et al. 2016; O’Dell et al. 2010). NE hotspots should play a role in tagand-capture by elevating local NE levels to activate $\beta$ adrenergic receptors, as well as by increasing glutamatergic activation of NMDA receptors. Both $\beta$-adrenergic activity and NMDA activity (in addition to dopamine D1/D5 receptor activity) are essential to "set the learning tag" for an initial weak memory, and $\beta$-adrenergic receptor activation is required during exposure to the modulating novel event occurring an hour later (Moncada et al. 2011). A particularly intriguing finding is that the behavioral tagging phenomenon requires the initial weak event and the subsequent novel event to occur in the same sensory modality, thereby activating the same general population of neurons (Ballarini et al. 2009). Likewise, Dunsmoor et al. (2015) found that fear conditioning enhanced memory for previously learned images only when those images were semantically related to a fear-conditioned category; when images of animals were fear-conditioned, memories for previously learned animals were enhanced, whereas when images of tools were fear-conditioned, memories for previously learned tools were enhanced. This is consistent with the local nature of NE hotspots and raises the 
Response/Mather et al.: Norepinephrine ignites local hotspots of neuronal excitation

Table R2. Data needed to test hypotheses and better understand arousal-priority or NE-glutamate interactions.

Can we measure GANE-proposed neurotransmitter mechanisms in laboratory animals?

Does NE interact with apical amplification priority signaling?

Is "network reset" a general motor-sensory or structurespecific effect?

How close in time does phasic arousal need to be to modulate the priority of another event?

Can we measure GANE-proposed neurotransmitter mechanisms in humans?

Test of NE hotspots in humans

Test Roozendaal et al. argument that NE effects on memory rely on the amygdala.

Inverted-U curve
Direct measurements of local glutamate levels and NE or $\beta$ adrenergic receptor activation levels in awake cortex with arousal/cue manipulations would make it possible to test our physiological GANE model. New techniques make it possible to track extra-synaptic glutamate activity (Okubo et al. 2010), and researchers are getting closer to being able to monitor levels of NE and G-couple protein receptor activation at spatial resolutions corresponding to a representational network

(Muller et al. 2014).

The Larkum \& Phillips hypothesis that NE modulates apical amplification in the output neurons of cortex as the mediator of top-down or cortico-cortical priority signals can be examined both in vitro and in vivo. Evidence for such gating would significantly expand the GANE model.

Immediate early genes with the ability to reveal two brain activation sequences separated by a temporal interval could test the reset (reorganizing)-versus-amplification effects of phasic LC activation. We predict evoked sensory representations would be enhanced and stabilized by phasic glutamatergic activation of LC, whereas hippocampal and possible prefrontal representations would be reconfigured. Tonic effects of NE would not evoke reset.

Initial behavioral data suggest that arousal induced by one event can modulate processing of other events occurring within a few seconds (see target article for review). Previous work indicates that glutamate activation of NMDA receptors decays slowly and can last hundreds of milliseconds (Lester et al. 1990), but more work is needed to quantify the timing of glutamate and NE actions at hotspots (allowing for formal modeling, as highlighted by Warren et al. in their commentary).

Advances in human magnetic resonance spectroscopy (MRS) enable the measurement of glutamate metabolites in vivo, but with poor spatial and temporal resolution. One straightforward test of GANE would be to examine whether an arousing stimulus can elicit a local, activity-dependent increase in glutamate levels for a prioritized stimulus.

During task-related fMRI involving an arousal $\times$ priority manipulation, trial-by-trial estimates of pupil dilation to the arousing stimulus could be used to scale BOLD responses in cortical representational regions underlying the high-priority stimulus. This would provide an estimate of how LC responses selectively modulate local cortical activity.

The fact that the hippocampus has many NE receptors suggests that NE can modulate memory consolidation in the hippocampus directly, without amygdala modulation (although $\mathrm{NE}$ release in the amygdala can lead to glutamatergic activation of the hippocampus, it does not directly increase NE in the hippocampus; see Fig. R2). A simple experiment would be to attempt to modulate consolidation of a hippocampally represented memory such as learning the context of a novel object by infusing NE into the hippocampus (as has been done with NE infused into the amygdala [Barsegyan et al. 2014])

A direct examination of inverted-U curve effects with $\mathrm{NE}$ would be of interest. It is not clear if the functional shift seen at high levels of arousal is uniquely, or even critically, due to high NE levels or is a multifactorial effect depending on co-activation of other systems. 
Response/Mather et al.: Norepinephrine ignites local hotspots of neuronal excitation

interesting question of just how widely the plasticity-related proteins stimulated via $\beta$-adrenergic receptor activation at NE hotspots modulate interconnected memory circuitries. The behavioral findings (Ballarini et al. 2009; Dunsmoor et al. 2015) suggest that they do not have an influence much beyond a local region that represents the same category or sensory modality of item. Although much still needs to be worked out about the potentially complementary roles of dopamine and norepinephrine on tag-andcapture phenomena, we believe that thinking about the local nature of the $\beta$-adrenergic activity induced by arousing modulatory events will be fruitful.

\section{R6. GANE amplification of prioritized representations during a "network reset"}

According to a prominent theory, NE release orchestrates a "network reset" that reorients attention and, consequently, reorganizes underlying representational networks during a sudden and unexpected change in environmental imperatives (Bouret \& Sara 2005; Sara \& Bouret 2012). We agree with Sara's perspective that GANE is complementary to the "reset" hypothesis. From the perspective of GANE, whether this type of reorienting occurs will depend on whether there are currently representations with high glutamatergic activity or not. If there are no current strongly active representations, both GANE and the network reset theory predict that the predominant effect of an increase in LC activity would be to enhance reorientation to new salient stimuli. However, when there is already a highly active representation, GANE predicts that an increase in LC activity will further enhance processing of that representation (e.g., Anderson et al. 2006; Knight \& Mather 2009; Sakaki et al. 2014a), rather than having a network reset effect. On the basis of these findings, in our target article we argued that the network reset perspective fails to account for the ability of arousal to enhance memory of preceding high-priority information. Bouret responded by suggesting that enhanced memory for a preceding event could be consistent with a network reset if, when an arousing event occurred, the preceding salient event was now represented in a qualitatively different way that was integrated with the arousing event.

Consistent with Bouret's argument that arousal enhances memory for preceding information when the preceding information is integrated with the arousing events, in fear/ evaluative conditioning paradigms events repeatedly followed by emotional outcomes acquire emotional properties (for a review, see Baeyens et al. 2005). Our previous research also demonstrated that when individuals are presented with neutral cues followed by emotional or neutral outcomes, emotional outcomes facilitate memory for neutral cues only when they are aware of the cue-outcome contingency (Mather \& Knight 2008; Sakaki et al. 2014b).

To address the important question raised by Bouret about whether arousal changes the nature of representations, future research should probe the effects of arousal on the specificity of mental and neuronal representations. At least one recent study suggests active sensory representations are strengthened, rather than altered, by noradrenergic system activation (Shakhawat et al. 2015). In addition, our findings suggest that emotional arousal enhances the veracity of the original representation, or detail memory, rather than gist alone (Sakaki et al. 2014a).

\section{R7. Alternative ways to trigger LC activity}

Although most of the target article focused on how emotionally arousing stimuli shape cognitive processing, nonemotional stimuli can also activate the LC and thereby influence cognition. In this section, we discuss how prediction errors, uncertainty, and competition each influence LC activity.

\section{R7.1. Prediction errors}

Prediction is a central feature of efficient cognitive processing. As described by Ferreira-Santos, GANE fits well with "predictive coding" frameworks of cognition: Sudden mismatches between predicted and actual sensory and affective inputs represent an important form of conflict and competition that can elicit arousal and LC activity. Supporting this view, pupil dilation has been linked to the occurrence of prediction errors (Braem et al. 2015; Preuschoff et al. 2011). Furthermore, in monkeys, phasic LC activity ceases to signal the occurrence of reward once the reward follows a specific action predictably (Sara \& Segal 1991). Other research also indicates that affect enhances prediction error responses (Vogel et al. 2015a) and that prediction errors are a fundamental component of generating interceptive feelings (Barrett \& Simmons 2015).

\section{R7.2. Uncertainty}

As pointed out by Nassar et al., as well as by Bouret, it is important to consider the purpose of having one level of arousal modulate cognitive processing differently than another level. When is it useful for cognitive processing to remain focused on previously salient information? And when will it be advantageous to be open to new prioritized information? Nassar and colleagues argue that during times of uncertainty, it is especially important not simply to focus on current prioritized cues, but to amplify incoming prioritized sensory information (Yu \& Dayan 2005). They review findings that pupil diameter is larger during periods of uncertainty than when expectations are reliable. Thus, tonically higher levels of $\mathrm{NE}$ should decrease the threshold for new salient stimuli to ignite hotspots. They suggest that older adults' deficits in learning under conditions of uncertainty may be linked to age-related declines in LC function.

\section{R7.3. Competition and conflict}

As highlighted by Phaf, there is much evidence that competition and conflict between representations induce arousal. These stimuli/events are also likely to produce hotspots, based on evidence that conflict, along with novelty, target detection, uncertainty, and performance errors, elicit LC activity (for reviews, see Berridge \& Waterhouse 2003; Nieuwenhuis et al. 2005a; Ullsperger et al. 2010; Yu \& Dayan 2005). Fundamentally, GANE predicts that any stimulus that activates the LC-NE system will produce hotspots in an activity-dependent manner, regardless of 
Response/Mather et al.: Norepinephrine ignites local hotspots of neuronal excitation

whether NE release is triggered by something emotional or not. If competition elicits arousal, it could very well be an effect driven by prediction errors (i.e., significant discrepancies between feedforward and feedback inputs; see sect. R7.1), initiating a network reset via the LC.

Phaf also discusses the distinct but complementary roles of theta and gamma oscillations in signaling and resolving stimulus conflict, respectively. According to Phaf, theta arises from conflict, is a substrate of arousal, and helps select dominant representations via intercortical communication. Subsequently, gamma oscillations facilitate a resetting and stabilization of "winning" representations. His description is consistent with Sara's empirical data. In her commentary, Sara describes evidence that stimulating the LC briefly suppresses gamma oscillations for $200 \mathrm{~ms}$, which is followed by a near doubling of the gamma power immediately afterward, as well as an increase in theta power (Sara 2015). Interestingly, in an early report of conflict activating LC, the absence of expected reward elicited a specific theta band increase $(\sim 7.7 \mathrm{~Hz})$ in hippocampus (Gray \& Ball 1970). This effect was later demonstrated to require forebrain norepinephrine (Gray et al. 1975). It could be useful to reexamine this theta signature of LC activation (for more recent support, see Walling et al. 2011) and its role in synchronizing activity for prioritized representations. Another interesting question is whether (as suggested in the target article) NE hotspots enhance local gamma power via a $\beta$-adrenergic pathway, thereby increasing selective attention.

\section{R8. Additional mechanistic considerations/ complications for GANE}

As noted by several commentators, GANE is necessarily a simplification of a complex reality. It does not, for example, incorporate the function of postsynaptic $\alpha_{2}$-receptors, the subthreshold input promoting role of $\alpha_{1}$-receptors, the synergistic role of $\alpha_{1}$ - with $\beta$-adrenergic receptors or recently described astrocytic functions of $\alpha_{1}$-receptors. The co-release of peptides from LC varicosities is not considered; neither is the probable role of other neuromodulators known to be elevated in various forms of arousal discussed. This is a beginning that will, ideally, lead to a more veridical model of cortical self-regulation that addresses how neurotransmitters released during arousal interact with local cortical conditions to modulate activity in flexible yet highly targeted ways.

\section{R8.1. Varied effects of adrenoreceptors}

As highlighted in several commentaries, the GANE model does not incorporate all known adrenoreceptor functions. These omissions include the role of postsynaptic $\alpha_{2}$-receptors that play important roles in the PFC (see commentaries by Abdallah et al. and Todd et al. and that also occur in other areas of neocortex (Venkatesan et al. 1996). Navarra \& Waterhouse and Gaucher \& Edeline point out that $\alpha_{1}$-adrenoreceptors have more varied actions, including synergism with $\beta$-adrenoreceptor effects, potentiation of effects on their own, and astrocytic action. In particular, they highlight that the role of $\alpha_{1}$-adrenoreceptor in sensory cortex may be facilitatory: When activated, these receptors appear to potentiate postsynaptic excitatory responses and can boost subthreshold inputs (for a review, see Berridge \& Waterhouse 2003). Furthermore, global astrocytic calcium waves are initiated via LC-NE activation of astrocytic $\alpha_{1}$-adrenoreceptors (Ding et al. 2013), consistent with a model in which LC-NE global effects recruit both $\alpha_{1^{-}}$and $\alpha_{2}$-adrenoreceptors.

\section{R8.2. Suppressive effects of NE in sensory regions}

Gaucher \& Edeline emphasize the suppressive actions of exogenous $\mathrm{NE}$ on processing in auditory cortex as being inconsistent with GANE. But their finding that a small population of auditory neurons encoding natural stimuli are enhanced by NE (Gaucher \& Edeline 2015) and contribute to discrimination is similar to newer findings in olfactory cortex that LC-NE modulation is essential for difficult natural odor discrimination and increases the stability of small distributed odor representations (Shakhawat et al. 2015), as predicted by GANE.

\section{R8.3. Differential effects of adrenergic receptors in prefrontal and posterior cortex}

Abdallah et al. highlight the differences between the actions of NE on classic sensory synapses in subcortical and posterior sensory regions and newly evolved circuits in layer 3 of the dorsolateral PFC (DLPFC). On the basis of animal and human research, they suggest hotspot effects are most likely to occur in sensory and limbic (e.g., amygdala, hippocampus) synapses where $\beta$-adrenoreceptors promote glutamate responses and long-term potentiation. In the PFC, in contrast to "classic" sensory areas, $\beta$-adrenoreceptor activation has been found to impair rather than enhance postsynaptic function via increased cAMP signaling (Arnsten et al. 2015a; Ramos \& Arnsten 2007). Like $\beta$-adrenoreceptors, $\alpha_{1^{-}}$and $\alpha_{2}$-adrenoreceptors also appear to have contrasting influence on neuronal activity in the PFC versus sensory cortices. Although $\alpha_{1}$-receptors enhance sensory neuron firing, they tend to impair PFC function and working memory (Ramos \& Arnsten 2007). On the other hand, whereas $\alpha_{2}$-receptors enhance inhibitory signals and suppress noisy activity in the posterior cortex, their activation strengthens dorsolateral PFC functional network connectivity and promotes working memory (Arnsten et al. 2012).

These inverted rules of adrenoreceptor function in the PFC have important implications for how GANE influences cognitive processing during sudden arousal. Although an arousal-induced surge of NE may disrupt working memory representations in the DLPFC (e.g., current event models), it should also transiently enhance the throughput of strong glutamatergic signals in the hippocampus (Brown et al. 2005). Therefore, DLPFC impairments may facilitate reorientation during arousal to information that has bottom-up salience and is associated with hotspots of high activity in sensory regions but not in PFC.

\section{R8.4. Relative timing of arousal and prioritization process}

The key distinction outlined in the previous section between the effects of $\mathrm{NE}$ in sensory cortices and limbic regions versus the PFC agrees well with the timing hypotheses proposed by Warren et al. In their commentary, 
Response/Mather et al.: Norepinephrine ignites local hotspots of neuronal excitation

Warren and colleagues present evidence that the relative strength of bottom-up and top-down (cognitive control) priority inputs changes rapidly within a single trial. Whereas bottom-up salience dominates the competition for mental resources early on, cognitive control processes take longer to develop and overcome the initial dominance of perceptual salience. Warren et al. suggest that this timevariant model of salience determines whether phasic arousal enhances or impairs task-relevant (but not perceptually salient) information.

Indeed, the GANE model predicts that arousal-induced NE release will bias competition in favor of whatever information has the highest priority at that moment. Experiencing arousal while a representation is highly active should strengthen that representation regardless of whether top-down goals or bottom-up salience prioritized the representation, because the representation was activated before moderate to high levels of $\mathrm{NE}$ could disrupt goal-directed processing in the PFC (Ramos \& Arnsten 2007). In contrast, the source of priority may matter more when experiencing arousal before a stimulus is perceived. Although prestimulus arousal should amplify the effects of bottom-up salience, it may diminish the effects of top-down priority if, as outlined in the previous section, working memory processes that help maintain and implement processing goals are impaired by the arousal (Ramos \& Arnsten 2007).

Data from our lab provide clear evidence that prestimulus arousal enhances the impact of bottom-up salience (Lee et al. 2014b; Sutherland \& Mather 2012), whereas poststimulus arousal enhances the impact of top-down prioritization (Lee et al. 2015; Sakaki et al. 2014a). Whether arousal enhances priority for the other two combinations remains to be seen. We have not yet tested scenarios in which something perceptually salient is followed by something arousing, but GANE would predict that as long as the representation associated with that perceptually salient item were still strongly active when arousal increased, it would benefit further from the arousal. In contrast, as outlined above, the situation in which arousal occurs before top-down prioritization occurs could show the reverse effect; insofar as arousal disrupts the ability of the PFC to prioritize an otherwise nonsalient stimulus, arousal should diminish the impact of top-down priority because the goal-relevant representation is not highly activated. Consistent with this, we have found that playing an emotional sound before a brief display of letters makes it harder for participants to selectively report the letters in the high point value color (Sutherland et al., under review). Given the impairing effects of high NE on DLPFC, for a prestimulus arousal to enhance processing of a goal-relevant item, the goal prioritization process would need to be relatively independent of the PFC, perhaps because it is automatic or habitual.

\section{R8.5. Inverted-U relationship between LC firing and cognitive selectivity}

Aston-Jones and Cohen (2005) proposed an inverted-U model of tonic NE function, in which low tonic LC activity promotes being inattentive and nonalert, moderate LC activity promotes being focused, and high tonic LC activity promotes distractibility. In their commentary, Navarra \& Waterhouse ask where along the inverted-
$\mathrm{U}$ function the glutamate-NE interactions proposed in GANE would operate. Their question is in part inspired by data from Devilbiss and Waterhouse (2000), who simultaneously administered glutamate and NE into in vitro rat barrel field cortex slices. They found that some cells showed a monotonic suppression of the excitatory postsynaptic response to glutamate, as NE increased. Other cells showed an inverse $\mathrm{U}$ shape, in which there were increasing glutamate-evoked discharges as NE increased to $5 \mathrm{nA}$, but then decreasing glutamateevoked discharges as $\mathrm{NE}$ tonic levels were further increased (10-30 nA). These findings suggest that tonic levels of NE modulate postsynaptic responses to glutamatergic input, which is quite interesting. In particular, it seems that high tonic levels of $\mathrm{NE}$ would quiet activity in neurons exhibiting this postsynaptic NE suppression, which could contribute to the general decrease in neural noise seen under arousal (one interesting side note is that they found that, unlike in layers II/III, NE-induced facilitation of glutamate-evoked responses was the predominant response in layer $\mathrm{V}$, which may be connected to the apical amplification ideas of Larkum \& Phillips). However, the in vitro preparation of the study eliminated the LC from the equation and so did not provide the opportunity to observe the glutamate-evoked local release of $\mathrm{NE}$ proposed in GANE. As outlined in Table R2, more research is needed measuring in vivo interactions of glutamate and NE, as the GANE hotspot mechanism involves interactions between the LC and distant cortical representations.

\section{R8.6. Individual differences}

Geva points out that tonic levels of arousal predict whether infants orient toward novel or familiar stimuli, and suggests that infancy is an interesting test case for GANE, as, unlike in later stages of development, infants lack an "established neural network set with implicit know-hows" that provide the glutamatergic priority signal necessary to ignite hotspots under arousal. Differences at the other end of life are also relevant, as Nassar et al. point out. Genetic variation in adrenergic receptors also may matter; Todd et al. make the case that ADRA2b deletion carriers have reduced inhibitory autoreceptor function.

\section{R9. Conclusions}

As evinced by the diverse range of commentary, the NE hotspot mechanism goes beyond just the emotion-cognition literature to explain how arousal influences different forms of cognitive selectivity. One of GANE's most vital contributions is that it showcases the ability of the cortex to regulate its own processing efficiency. Such local control of cognition represents a fundamental mechanism of adaptive brain function that has the potential to explain a variety of cognitive phenomena. As GANE exemplifies, synaptic activity is not just passively modified by neuromodulators. Instead, under situations of arousal that demand our attention, such as threat or excitement, salient brain signals recruit the ingredients necessary to form lasting memories. 
References/Mather et al.: Norepinephrine ignites local hotspots of neuronal excitation

\section{References}

[The letters “a” and " $r$ " before author's initials stand for target article and response references, respectively]

Abercrombie, H. C., Speck, N. S. \& Monticelli, R. M. (2006) Endogenous cortisol elevations are related to memory facilitation only in individuals who are emotionally aroused. Psychoneuroendocrinology 31(2):187-96. [BR]

Abraham, W. C. \& Tate, W. P. (1997) Metaplasticity: A new vista across the field of synaptic plasticity. Progress in Neurobiology 52(4):303-23. [BAS]

Abraham, W. C. \& Williams, J. M. (2008) LTP maintenance and its protein synthesisdependence. Neurobiology of Learning and Memory 89(3):260-68. [aMM]

Ackermann, S. \& Rasch, B. (2014) Differential effects of non-REM and REM sleep on memory consolidation? Current Neurology and Neuroscience Reports 14:430. doi: 10.1007/s11910-013-0430-8. [AB]

Agmo, A. (1999) Sexual motivation: An inquiry into events determining the occurrence of sexual behavior. Behavioural Brain Research 105(1):129-50. [HM]

Albrecht, S. \& Carbon, C. C. (2014) The fluency amplification model: Fluent stimuli show more intense but not evidently more positive evaluations. Acta Psychologica 148:195-203. [C-CC, rMM]

Alger, B. E. \& Nicoll, R. A. (1980) Epileptiform burst afterhyperolarization: Calcium-dependent potassium potential in hippocampal CAl pyramidal cells. Science 210(4474):1122-24. [aMM]

Alnæs, D., Sneve, M. H., Espeseth, T., Endestad, T., van de Pavert, S. H. P. \& Laeng, B. (2014) Pupil size signals mental effort deployed during multiple object tracking and predicts brain activity in the dorsal attention network and the locus coeruleus. Journal of Vision 14(4). doi: 10.1167/14.4.1. [aMM]

Altman, J. (1963) Autoradiographic investigation of cell proliferation in the brains of rats and cats. The Anatomical Record 145:573-91. [CH]

Amaral, D. G. (1978) A Golgi study of cell types in the hilar region of the hippocampus in the rat. Journal of Comparative Neurology 182:851-914. [CH]

Amaral, D. G., Behniea, H. \& Kelly, J. L. (2003) Topographic organization of projections from the amygdala to the visual cortex in the macaque monkey. Neuroscience 118(4):1099-120. [aMM]

Amaral, D. G., Scharfman, H. E. \& Lavenex, P. (2007) The dentate gyrus: Fundamental neuroanatomical organization (dentate gyrus for dummies). Progress in Brain Research 163:3-22. [CH]

Amaral, D. G. \& Witter, M. P. (1989) The three-dimensional organization of the hippocampal formation: A review of anatomical data. Neuroscience 31:57191. [CH]

Anderson, A. K. (2005) Affective influences on the attentional dynamics supporting awareness. Journal of Experimental Psychology: General 134(2):258-81. doi: 10.1037/0096-3445.134.2.258. [aMM]

Anderson, A. K., Christoff, K., Panitz, D., De Rosa, E. \& Gabrieli, J. D. (2003) Neural correlates of the automatic processing of threat facial signals. The Journal of Neuroscience 23(13):5627-33. [rMM]

Anderson, A. K. \& Phelps, E. A. (2001) Lesions of the human amygdala impair enhanced perception of emotionally salient events. Nature 411:305-309. [HDC]

Anderson, A. K., Wais, P. E. \& Gabrieli, J. D. E. (2006) Emotion enhances remembrance of neutral events past. Proceedings of the National Academy of Sciences of the United States of America 103(5):1599-604. doi: 10.1073/ pnas.0506308103. [arMM, BAS]

Andrés, M. E., Bustos, G. \& Gysling, K. (1993) Regulation of $\left[{ }^{3} \mathrm{H}\right]$ norepinephrine release by $\mathrm{N}$-methyl-D-aspartate receptors in minislices from the dentate gyrus and the CA1-CA3 area of the rat hippocampus. Biochemical Pharmacology 46:1983-87. [CH]

Aracri, P., Banfi, D., Pasini, M. E., Amadeo, A. \& Becchetti, A. (2015) Hypocretin (orexin) regulates glutamate input to fast-spiking interneurons in layer $\mathrm{V}$ of the Fr2 region of the murine prefrontal cortex. Cerebral Cortex 25:1330-47. doi: 10.1093/cercor/bht326. [AB]

Arnsten, A. F. (2015) Stress weakens prefrontal networks: Molecular insults to higher cognition. Nature Neuroscience 18:1376-85. [CGA]

Arnsten, A. F. \& Cai, J. (1993) Postsynaptic alpha-2 receptor stimulation improves memory in aged monkeys: Indirect effects of yohimbine versus direct effects of clonidine. Neurobiology of Aging 14(6):597-603. [aMM]

Arnsten, A. F. \& Goldman-Rakic, P. S. (1985) Alpha 2-adrenergic mechanisms in prefrontal cortex associated with cognitive decline in aged nonhuman primates. Science 230(4731):1273-76. [aMM]

Arnsten, A. F., Raskind, M. A., Taylor, F. B. \& Connor, D. F. (2015a) The effects of stress exposure on prefrontal cortex: Translating basic research into successful treatments for post-traumatic stress disorder. Neurobiology of Stress 1:89-99. [rMM]

Arnsten, A. F., Steere, J. C. \& Hunt, R. D. (1996) The contribution of alpha 2noradrenergic mechanisms of prefrontal cortical cognitive function: Potential significance for attention-deficit hyperactivity disorder. Archives of General Psychiatry 53:448-55. [RMT]
Arnsten, A. F., Wang, M. J. \& Paspalas, C. D. (2012) Neuromodulation of thought: Flexibilities and vulnerabilities in prefrontal cortical network synapses. Neuron 76(1):223-39. [MEL, rMM]

Arnsten, A. F. T. (2009) Stress signalling pathways that impair prefrontal cortex structure and function. Nature Reviews Neuroscience 10(6):410-22. Available at: http://doi.org/10.1038/nrn2648. [SB]

Arnsten, A. F. T. (2011) Catecholamine influences on dorsolateral prefrontal cortical networks. Biological Psychiatry 69(12):e89-e99. Available at: http://www.sciencedirect.com/science/article/pii/S0006322311001193. [arMM]

Arnsten, A. F. T. \& Goldman-Rakic, P. S. (1984) Selective prefrontal cortical projections to the region of the locus coeruleus and raphe nuclei in the rhesus monkey. Brain Research 306(1/2):9-18. Available at: http://dx.doi.org/10.1016/ 0006-8993(84)90351-2. [aMM]

Arnsten, A. F. T., Raskind, M., Taylor, F. B. \& Connor, D. F. (2015b) The effects of stress exposure on prefrontal cortex: Translating basic research into successful treatments for post-traumatic stress disorder. Neurobiology of Stress 1:89-99. [CGA]

Arnsten, A. F. T. \& Wang, M. (2016) Targeting prefrontal cortical systems for drug development: Potential therapies for cognitive disorders. Annual Review of Pharmacology and Toxicology 56:339-60. doi: 10.1146/annurev-pharmtox010715-103617. [CGA]

Arnsten, A. F. T., Wang, M. \& Paspalas, C. D. (2012) Neuromodulation of thought: Flexibilities and vulnerabilities in prefrontal cortical network synapses. Neuron 76(1):223-39. [CGA]

Astafiev, S. V., Snyder, A. Z., Shulman, G. L. \& Corbetta, M. (2010) Comment on "Modafinil shifts human locus coeruleus to low-tonic, high-phasic activity during functional MRI" and "Homeostatic sleep pressure and responses to sustained attention in the suprachiasmatic area." Science 328(5976):309. [aMM]

Aston-Jones, G. \& Bloom, F. E. (1981) Nonrepinephrine-containing locus coeruleus neurons in behaving rats exhibit pronounced responses to non-noxious environmental stimuli. The Journal of Neuroscience 1(8):887-900. [aMM, SB, SJS]

Aston-Jones, G. \& Cohen, J. D. (2005) An integrative theory of locus coeruleusnorepinephrine function: Adaptive gain and optimal performance. Annual Review of Neuroscience 28:403-50. http://doi.org/10.1146/annurev.neuro.28. 061604.135709. [arMM, SB, EE, MRN]

Aston-Jones, G., Rajkowski, J. \& Cohen, J. (1999) Role of locus coeruleus in attention and behavioral flexibility. Biological Psychiatry 46(9):1309-20. [arMM, RN]

Aston-Jones, G., Rajkowski, J. \& Kubiak, P. (1997) Conditioned responses of monkey locus coeruleus neurons anticipate acquisition of discriminative behavior in a vigilance task. Neuroscience 80(3):697-715. Available at: http://www.ncbi.nlm. nih.gov/pubmed/9276487. [SJS]

Aston-Jones, G., Rajkowski, J., Kubiak, P. \& Alexinsky, T. (1994) Locus coeruleus neurons in monkey are selectively activated by attended cues in a vigilance task. The Journal of Neuroscience 14(7):4467-80. [BAS]

Aston-Jones, G., Shipley, M. T., Chouvet, G., Ennis, M., van Bockstaele, E. J., Pieribone, V. A., Shiekhattar, R., Akaoka, H., Drolet, G. \& Astier, B. (1991) Afferent regulation of locus coeruleus neurons: Anatomy, physiology and pharmacology. Progress in Brain Research 88:47-75. [SB]

Awh, E., Belopolsky, A. V. \& Theeuwes, J. (2012) Top-down versus bottom-up attentional control: A failed theoretical dichotomy. Trends in Cognitive Sciences 16(8):437-43. [aMM]

Bach, D. R., Talmi, D., Hurlemann, R., Patin, A. \& Dolan, R. J. (2011) Automatic relevance detection in the absence of a functional amygdala. Neuropsychologia 49(5):1302-305. [rMM]

Bachmann, T. \& Hudetz, A. G. (2014) It is time to combine the two main traditions in the research on the neural correlates of consciousness: $\mathrm{C}=\mathrm{L} \times \mathrm{D}$. Frontiers in Psychology 5:Article 940. doi: 10.3389/fpsyg.2014.00940. [MEL]

Baeyens, F., Field, A. P. \& Houwer, J. D. (2005) Associative learning of likes and dislikes: Some current controversies and possible ways forward. Cognition and Emotion 19(2):161-74. [rMM]

Bakker, A., Kirwan, C. B., Miller, M. \& Stark, C. E. L. (2008) Pattern separation in the human hippocampal CA3 and dentate gyrus. Science 319(5870):1640-42. [aMM]

Ballarini, F., Moncada, D., Martinez, M. C., Alen, N. \& Viola, H. (2009) Behavioral tagging is a general mechanism of long-term memory formation. Proceedings of the National Academy of Sciences of the United States of America 106 (34):14599-604. [MR, rMM]

Baluch, F. \& Itti, L. (2011) Mechanisms of top-down attention. Trends in Neurosciences 34(4):210-24. [aMM]

Bangasser, D. \& Valentino, R. (2012) Sex differences in molecular and cellular substrates of stress. Cellular and Molecular Neurobiology 32(5):709-23. doi: 10.1007/s10571-012-9824-4. [aMM]

Barnacle, G. E., Schaefer, A., Tsivilis, D. \& Talmi, D. (2015) Understanding Emotional Memory:Cognitive Factors. University of Manchester, School of psychological sciences. [DT]

Barnacle, Schaefer, Tsvilis \& Talmi, in preparation. $\quad[\mathrm{rMM}]$ 
Barrett, L. F. \& Bar, M. (2009) See it with feeling: Affective predictions during object perception. Philosophical Transactions of the Royal Society B: Biological Sciences 364:1325-34. doi: 10.1098/rstb.2008.0312. [FF-S]

Barrett, L. F. \& Kensinger, E. A. (2010) Context is routinely encoded during emotion perception. Psychological Science 21:595-99. [KK]

Barrett, L. F., Mesquita, B. \& Gendron, M. (2011) Context in emotion perception. Current Directions in Psychological Science 20:286-90. [KK]

Barrett, L. F. \& Simmons, W. K. (2015) Interoceptive predictions in the brain. Nature Reviews Neuroscience 16:419-29. doi: 10.1038/nrn3950. [FF-S, $\mathrm{rMM}]$

Barsegyan, A., McGaugh, J. L. \& Roozendaal, B. (2014) Noradrenergic activation of the basolateral amygdala modulates the consolidation of object-in-context recognition memory. Frontiers in Behavioral Neuroscience 8:Article 160. [arMM, BR]

Bassant, M. H., Ennouri, K. \& Lamour, Y. (1990) Effects of iontophoretically applied monoamines on somatosensory cortical neurons of unanesthetized rats. Neuroscience 39:431-39. [QG]

Bauer, F., Cheadle, S. W., Parton, A., Müller, H. J. \& Usher, M. (2009) Gamma flicker triggers attentional selection without awareness. Proceedings of the National Academy of Sciences of the United States of America 106:1666-71. [RHP]

Bayer, S. A., Yackel, J. W. \& Puri, P. S. (1982) Neurons in the rat dentate gyrus granular layer substantially increase during juvenile and adult life. Science 216:890-92. [CH]

Beaudet, A. \& Descarries, L. (1978) The monoamine innervation of rat cerebral cortex: Synaptic and nonsynaptic axon terminals. Neuroscience 3(10):851-60. $[\mathrm{aMM}]$

Bechara, A., Damasio, H., Damasio, A. R. \& Lee, G. P. (1999) Different contributions of the human amygdala and ventromedial prefrontal cortex to decisionmaking. Journal of Neuroscience 19(13):5473-81. [rMM]

Bechara, A., Tranel, D., Damasio, H., Adolphs, R., Rockland, C. \& Damasio, A. R. (1995) Double dissociation of conditioning and declarative knowledge relative to the amygdala and hippocampus in humans. Science 269(5227):1115-18. [BR, rMM]

Beck, D. M. \& Kastner, S. (2009) Top-down and bottom-up mechanisms in biasing competition in the human brain. Vision Research 49(10):1154-65. doi: 10.1016/ j.visres.2008.07.012. [aMM]

Becker, M. W. \& Leinenger, M. (2011) Attentional selection is biased toward moodcongruent stimuli. Emotion 11:1248-54. [KK]

Bekar, L. K., Wei, H. S. \& Nedergaard, M. (2012) The locus coeruleus-norepinephrine network optimizes coupling of cerebral blood volume with oxygen demand. Journal of Cerebral Blood Flow and Metabolism 32:2135-45. [aMM]

Beldjoud, H., Barsegyan, A. \& Roozendaal, B. (2015) Noradrenergic activation of the basolateral amygdala enhances object recognition memory and induces chromatin remodeling in the insular cortex. Frontiers in Behavioral Neuroscience 9:108. [BR]

Benavides-Piccione, R., Arellano, J. I. \& DeFelipe, J. (2005) Catecholaminergic innervation of pyramidal neurons in the human temporal cortex. Cerebral Cortex 15(10):1584-91. doi: 10.1093/cercor/bhi036. [aMM]

Bennion, K. A., Ford, J. H., Murray, B. D. \& Kensinger, E. A. (2013) Oversimplification in the study of emotional memory. Journal of the International Neuropsychological Society 19(9):953-61. [BR]

Berg, D. K. (2011) Timing is everything, even for cholinergic control. Neuron 71:68. doi: 10.1016/j.neuron.2011.06.029. [AB]

Bergado, J. A., Lucas, M. \& Richter-Levin, G. (2011) Emotional tagging: A simple hypothesis in a complex reality. Progress in Neurobiology 94(1):64-76. doi: 10.1016/j.pneurobio.2011.03.004. [aMM]

Berridge, C. W. \& Abercrombie, E. D. (1999) Relationship between locus coeruleus discharge rates and rates of norepinephrine release within neocortex as assessed by in vivo microdialysis. Neuroscience 93:1263-70. [RN]

Berridge, C. \& Foote, S. (1991) Effects of locus coeruleus activation on electroencephalographic activity in neocortex and hippocampus. The Journal of Neuroscience $11(10): 3135-45 . \quad$ [aMM, QG]

Berridge, C., Page, M., Valentino, R. \& Foote, S. (1993) Effects of locus coeruleus inactivation on electroencephalographic activity in neocortex and hippocampus. Neuroscience 55(2):381-93. [aMM, QG]

Berridge, C. W., Schmeichel, B. E. \& Espana, R. A. (2012) Noradrenergic modulation of wakefulness/arousal. Sleep Medicine Reviews 16(2):187-97. doi: 10.1016/j.smrv.2011.12.003. [aMM]

Berridge, C. W. \& Waterhouse, B. D. (2003) The locus coeruleus-noradrenergic system: Modulation of behavioral state and state-dependent cognitive processes. Brain Research Reviews 42(1):33-84. doi: 10.1016/s0165-0173(03) 00143-7. [arMM, SB, CH, RN]

Bickler, P. E. \& Hansen, B. M. (1996) $\alpha_{2}$-Adrenergic agonists reduce glutamate release and glutamate receptor-mediated calcium changes in hippocampal slices during hypoxia. Neuropharmacology 35(6):679-87. [aMM]
Bienenstock, E. L., Cooper, L. N. \& Munro, P. W. (1982) Theory for the development of neuron selectivity: Orientation specificity and binocular interaction in visual cortex. The Journal of Neuroscience 2(1):32-48. [BAS]

Bigham, M. H. \& Lidow, M. S. (1995) Adrenergic and serotonergic receptors in aged monkey neocortex. Neurobiology of Aging 16(1):91-104. [aMM]

Bindra, D. (1974) A motivational view of learning, performance, and behavior modification. Psychological Review 81(3):199. [HM]

Birnbaum, S. B., Yuan, P., Wang, M., Vijayraghavan, S., Bloom, A., Davis, D. Gobeske, K. T., Sweatt, J. D., Manji, H. K. \& Arnsten, A. F. T. (2004) Protein kinase $\mathrm{C}$ overactivity impairs prefrontal cortical regulation of working memory. Science 306:882-84. [CGA]

Bishop, S. J. (2007) Neurocognitive mechanisms of anxiety: An integrative account. Trends in Cognitive Sciences 11(7):307-16. [aMM]

Bliss, T. V. P. \& Collingridge, G. L. (1993) A synaptic model of memory: Long-term potentiation in the hippocampus. Nature 361(6407):31-39. [aMM]

Bloomer, W. A., VanDongen, H. M. \& VanDongen, A. M. (2008) Arc/Arg3.1 translation is controlled by convergent $N$-methyl-D-aspartate and $\mathrm{G}_{\mathrm{s}}$-coupled receptor signaling pathways. Journal of Biological Chemistry 283(1):582-92. [aMM]

Bocanegra, B. R. \& Zeelenberg, R. (2009) Dissociating emotion-induced blindness and hypervision. Emotion 9(6):865-73. doi: 10.1037/a0017749. [aMM]

Böckler, A., Alpay, G. \& Stürmer, B. (2011) Accessory stimuli affect the emergence of conflict, not conflict control: A Simon-task ERP study. Experimental Psychology 58:102-109. doi: 10.1027/1618-3169/a000073. [CMW]

Bosman, C. A., Lansink C. S. \& Pennartz, C. M. (2014) Functions of gamma-band synchronization in cognition: From single circuits to functional diversity across cortical and subcortical systems. European Journal of Neuroscience 39:198299. [SJS]

Both, S., Boxtel, G., Stekelenburg, J., Everaerd, W. \& Laan, E. (2005) Modulation of spinal reflexes by sexual films of increasing intensity. Psychophysiology 42(6):726-31. [HM]

Both, S., Spiering, M., Everaerd, W. \& Laan, E. (2004) Sexual behavior and responsiveness to sexual stimuli following laboratory-induced sexual arousal. Journal of Sex Research 41(3):242-58. [HM]

Botvinick, M. M., Nystrom, L. E., Fissell, K., Carter, C. S. \& Cohen, J. D. (1999) Conflict monitoring versus selection-for-action in anterior cingulate cortex. Nature 402(6758):179-81. [aMM]

Bouret, S., Duvel, A., Onat, S. \& Sara, S. J. (2003) Phasic activation of locus ceruleus neurons by the central nucleus of the amygdala. The Journal of Neuroscience 23(8):3491-97. [aMM]

Bouret, S. \& Richmond, B. J. (2015) Sensitivity of locus ceruleus neurons to reward value for goal-directed actions. The Journal of Neuroscience 35(9):4005-14. doi 10.1523/jneurosci.4553-14.2015. [aMM, RG]

Bouret, S. \& Sara, S. J. (2002) Locus coeruleus activation modulates firing rate and temporal organization of odour-induced single-cell responses in rat piriform cortex. European Journal of Neuroscience 16:2371-82. [QG]

Bouret, S. \& Sara, S. J. (2004) Reward expectation, orientation of attention and locus coeruleus-medial frontal cortex interplay during learning. European Journal of Neuroscience 20(3):791-802. Available at: http://doi.org/10.1111/j.1460-9568. 2004.03526.x. [SB, SJS]

Bouret, S. \& Sara, S. J. (2005) Network reset: A simplified overarching theory of locus coeruleus noradrenaline function. Trends in Neurosciences 28(11):574-82. Available at: http://dx.doi.org/10.1016/j.tins.2005.09.002. [arMM, SB, SJS]

Bradley, M. M., Costa, V. D. \& Lang, P. J. (2015) Selective looking at natural scenes: Hedonic content and gender. International Journal of Psychophysiology 98 (1):54-58. [rMM]

Bradley, M. M., Greenwald, M. K., Petry, M. C. \& Lang, P. J. (1992) Remembering pictures: Pleasure and arousal in memory. Journal of Experimental Psychology: Learning, Memory and Cognition 18(2):379-90. [HM]

Bradley, M. M., Miccoli, L., Escrig, M. A. \& Lang, P. J. (2008) The pupil as a measure of emotional arousal and autonomic activation. Psychophysiology 45(4):602-607. doi: 10.1111/j.1469-8986.2008.00654.x. [AM, rMM]

Braem, S., Coenen, E., Bombeke, K., van Bochove, M. E. \& Notebaert, W. (2015) Open your eyes for prediction errors. Cognitive, Affective and Behavioral Neuroscience 15(2):374-80. [rMM]

Brede, M., Philipp, M., Knaus, A., Muthig, V. \& Hein, L. (2004) $\alpha_{2}$-Adrenergic receptor subtypes - Novel functions uncovered in gene-targeted mouse models. Biology of the Cell 96(5):343-48. [aMM]

Bremner, J. D., Innis, R. B., Ng, C. K., Staib, L. H., Salomon, R. M., Bronen, R. A., Duncan, J., Southwick, S. M., Krystal, J. H., Rich, D., Zubal, G., Dey, H., Soufer, R. \& Charney, D. S. (1997) Positron emission tomography measurement of cerebral metabolic correlates of yohimbine administration in combatrelated posttraumatic stress disorder. Archives of General Psychiatry 54:24654. [CGA]

Bressler, S. L. \& Menon, V. (2010) Large-scale brain networks in cognition: Emerging methods and principles. Trends in Cognitive Sciences 14(6):27790. [aMM] 
Briand, L. A., Gritton, H., Howe, W. M., Young, D. A. \& Sarter, M. (2007) Modulators in concert for cognition: Modulator interactions in the prefrontal cortex. Progress in Neurobiology 83(2):69-91. [aMM]

Brown, R. A. M., Walling, S. G., Milway, J. S. \& Harley, C. W. (2005) Locus ceruleus activation suppresses feedforward interneurons and reduces beta-gamma electroencephalogram frequencies while it enhances theta frequencies in rat dentate gyrus. Journal of Neuroscience 25(8):1985-91. doi: 10.1053/jneurosci.4307-04-2005. [arMM]

Bryant, J. \& Miron, D. (2003) Excitation-transfer theory and three-factor theory of emotion. In: Communication and emotion: Essays in honor of Dolf Zillmann, ed. J. Bryant, D. Roskos-Ewoldsen \& J. Cantor, pp. 31-59. Erlbaum. [KK]

Bryant, R. A., McGrath, C. \& Felmingham, K. L. (2013) The roles of noradrenergic and glucocorticoid activation in the development of intrusive memories. PLoS ONE 8(4):e62675. Available at: http://www.ncbi.nlm.nih.gov/pubmed/ 23658640. [RH]

Buchanan, T. W. \& Lovallo, W. R. (2001) Enhanced memory for emotional material following stress-level cortisol treatment in humans. Psychoneuroendocrinology 26 (3):307-17. Available at: http://www.ncbi.nlm.nih.gov/pubmed/11166493. [RH]

Burban, A., Faucard, R., Armand, V., Bayard, C., Vorobjev, V. \& Arrang, J.-M. (2010) Histamine potentiates $N$-methyl-D-aspartate receptors by interacting with an allosteric site distinct from the polyamine binding site. Journal of Pharmacology and Experimental Therapeutics 332(3):912-21. [aMM]

Burke, J. F., Zaghloul, K. A., Jacobs, J., Williams, R. B., Sperling, M. R., Sharan, A D. \& Kahana, M. J. (2013) Synchronous and asynchronous theta and gamma activity during episodic memory formation. The Journal of Neuroscience 33 (1):292-304. [aMM]

Bush, G., Luu, P. \& Posner, M. I. (2000) Cognitive and emotional influences in anterior cingulate cortex. Trends in Cognitive Sciences 4(6):215-22. [aMM]

Buzsáki, G. \& Wang, X.-J. (2012) Mechanisms of gamma oscillations. Annual Review of Neuroscience 35(1):203-25. doi: 10.1146/annurev-neuro-062111-150444. [aMM]

Cahill, L., Babinsky, R., Markowitsch, H. J. \& McGaugh, J. L. (1995) The amygdala and emotional memory. Nature 377(6547):295-96. [BR]

Cahill, L., Gorski, L. \& Le, K. (2003) Enhanced human memory consolidation with post-learning stress: Interaction with the degree of arousal at encoding. Learning and Memory 10(4):270-74. doi: 10.1101/lm.62403. [aMM]

Cahill, L., Prins, B., Weber, M. \& McGaugh, J. L. (1994) Beta-adrenergic activation and memory for emotional events. Nature 371(6499):702-704. [BR]

Cai, W., Chen, T., Ryali, S., Kochalka, J., Li, C.-S. R. \& Menon, V. (2015) Causal interactions within a frontal-cingulate-parietal network during cognitive control: Convergent evidence from a multisite-multitask investigation. Cerebral Cortex 26(5):2140-53. doi: 10.1093/cercor/bhv046. [aMM]

Callejas, A., Lupiáñez, J., Funes, M. J. \& Tudela, P. (2005) Modulations among the alerting, orienting and executive control networks. Experimental Brain Research 167:27-37. doi: 10.1007/s00221-005-2365-z. [CMW]

Callejas, A., Lupiánez, J. \& Tudela, P. (2004) The three attentional networks: On their independence and interactions. Brain and Cognition 54(3):225-27. [CMW]

Campbell, B. A., Wood, G. \& McBride, T. (1997) Origins of orienting and defensive responses: An evolutionary perspective. In: Attention and orienting: Sensory and motivational processes, ed. P. J. Lang, R. F. Simons, M. Balaban \& R. Simons, pp. 41-67. Psychology Press. [HM]

Canli, T., Zhao, Z., Brewer, J., Gabrieli, J. D. \& Cahill, L. (2000) Event-related activation in the human amygdala associates with later memory for individual emotional experience. The Journal of Neuroscience 20(19):RC99. [BR]

Canolty, R. T., Edwards, E., Dalal, S. S., Soltani, M., Nagarajan, S. S., Kirsch, H. E., Berger, M. S., Barbaro, N. M. \& Knight, R. T. (2006) High gamma power is phase-locked to theta oscillations in human neocortex. Science 313(5793):162628. doi: 10.1126/science.1128115. [aMM]

Canolty, R. T. \& Knight, R. T. (2010) The functional role of cross-frequency coupling. Trends in Cognitive Sciences 14(11):506-15. Available at: http://dx.doi. org/10.1016/j.tics.2010.09.001. [aMM]

Cantor, J. R., Zillmann, D. \& Bryant, J. (1975) Enhancement of experienced sexual arousal in response to erotic stimuli through misattribution of unrelated residual excitation. Journal of Personality and Social Psychology 32(1):69. [JRH]

Carbon, C. C. (2014) Understanding human perception by human-made illusions. Frontiers in Human Neuroscience 8(566):1-6. [C-CC]

Cardin, J. A., Carlen, M., Meletis, K., Knoblich, U., Zhang, F., Deisseroth, K., Tsai, L.-H. \& Moore, C. I. (2009) Driving fast-spiking cells induces gamma rhythm and controls sensory responses. Nature 459(7247):663-67. doi: 10.1038/ nature08002. [aMM]

Carlen, M., Meletis, K., Siegle, J. H., Cardin, J. A., Futai, K., Vierling-Claassen, D., Ruhlmann, C., Jones, S. R., Deisseroth, K., Sheng, M., Moore, C. I. \& Tsai, L. H. (2012) A critical role for NMDA receptors in parvalbumin interneurons for gamma rhythm induction and behavior. Molecular Psychiatry 17(5):537-48. Available at: http://www.nature.com/mp/journal/v17/n5/suppinfo/mp201131s1. html. [aMM]
Carrasco, G. A. \& Van de Kar, L. D. (2003) Neuroendocrine pharmacology of stress European Journal of Pharmacology 463(1-3):235-72. Available at: http://dx.doi org/10.1016/S0014-2999(03)01285-8. [aMM]

Carstensen, L. L., Fung, H. H. \& Charles, S. T. (2003) Socioemotional selectivity theory and the regulation of emotion in the second half of life. Motivation and Emotion 27:103-23. [KK]

Carter, M. E., Yizhar, O., Chikahisa, S., Nguyen, H., Adamantidis, A., Nishino, S., Deisseroth, K. \& de Lecea, L. (2010) Tuning arousal with optogenetic modulation of locus coeruleus neurons. Nature Neuroscience 13(12):1526-33. Available at: http://doi.org/10.1038/nn.2682. [arMM, SB]

Cavanagh, J. F., Eisenberg, I., Guitart-Masip, M., Huys, Q. \& Frank, M. J. (2013) Frontal theta overrides Pavlovian learning biases. The Journal of Neuroscience 33(19):8541-48. [HM]

Cedarbaum, J. M. \& Aghajanian, G. K. (1978) Afferent projections to the rat locus coeruleus as determined by a retrograde tracing technique. Journal of Comparative Neurology 178(1):1-15. [rMM]

Cerf, M., Frady, E. P. \& Koch, C. (2009) Faces and text attract gaze independent of the task: Experimental data and computer model. Journal of Vision 9(12):10. [aMM]

Chalifoux, J. R. \& Carter, A. G. (2011) Glutamate spillover promotes the generation of NMDA spikes. The Journal of Neuroscience 31(45):16435-46. [MEL]

Chamberlain, S. R. \& Robbins, T. W. (2013) Noradrenergic modulation of cognition: Therapeutic implications. Journal of Psychopharmacology 27(8):694-718. [aMM]

Chambers, R. A., Bremner, J. D., Moghaddam, B., Southwick, S. M., Charney, D. S. \& Krystal, J. H. (1999) Glutamate and post-traumatic stress disorder: Toward a psychobiology of dissociation. Seminars in Clinical Neuropsychiatry 4:27481. [CGA]

Chandler, D. J., Gao, W.-J. \& Waterhouse, B. D. (2014) Heterogeneous organization of the locus coeruleus projections to prefrontal and motor cortices. Proceedings of the National Academy of Sciences of the United States of America 111 (18):6816-21. [aMM, QG]

Chandler, D. J., Lamperski, C. S. \& Waterhouse, B. D. (2013) Identification and distribution of projections from monoaminergic and cholinergic nuclei to functionally differentiated subregions of prefrontal cortex. Brain Research 1522:38-58. [QG]

Chang, C. C., Goshima, Y. \& Misu, Y. (1986) Evidence for the existence of stereoselective presynaptic $\beta_{1}$-adrenoceptors on noradrenergic and dopaminergic neurons in the rat hypothalamus. The Japanese Journal of Pharmacology 42 (3):447-49. [aMM]

Chan-Palay, V. \& Asan, E. (1989) Quantitation of catecholamine neurons in the locus coeruleus in human brains of normal young and older adults and in depression. The Journal of Comparative Neurology 287:357-72. [MRN]

Chau, L. S. \& Galvez, R. (2012) Amygdala's involvement in facilitating associative learning-induced plasticity: A promiscuous role for the amygdala in memory acquisition. Frontiers in Integrative Neuroscience 6:92. doi: 10.3389/ fnint.2012.00092. [aMM]

Chen, F. J. \& Sara, S. J. (2007) Locus coeruleus activation by foot shock or electrical stimulation inhibits amygdala neurons. Neuroscience 144(2):472-81. [aMM]

Cheun, J. E. \& Yeh, H. H. (1992) Modulation of GABAA receptor-activated current by norepinephrine in cerebellar Purkinje cells. Neuroscience 51:951-60. $[\mathrm{RN}]$

Choi, J. M., Padmala, S. \& Pessoa, L. (2012) Impact of state anxiety on the interaction between threat monitoring and cognition. NeuroImage 59(2):1912-23. [aMM]

Christianson, S. Å. \& Mjörndal, T. (1985) Adrenalin, emotional arousal and memory. Scandinavian Journal of Psychology 26:237-48. [AM]

Cichon, J. \& Gan, W. B. (2015) Branch-specific dendritic $\mathrm{Ca}^{2+}$ spikes cause persistent synaptic plasticity. Nature 520(7546):180-85. [MEL]

Cirelli, C., Huber, R., Gopalakrishnan, A., Southard, T. L. \& Tononi, G. (2005) Locus ceruleus control of slow-wave homeostasis. The Journal of Neuroscience 25(18):4503-11. doi: 10.1523/jneurosci.4845-04.2005. [aMM]

Cirelli, C., Pompeiano, M. \& Tononi, G. (1996) Neuronal gene expression in the waking state: A role for the locus coeruleus. Science 274(5290):1211-15. [aMM]

Cirelli, C. \& Tononi, G. (2000) Differential expression of plasticity-related genes in waking and sleep and their regulation by the noradrenergic system. The Journal of Neuroscience 20(24):9187-94. [aMM]

Clark, A. (2013) Whatever next? Predictive brains, situated agents, and the future of cognitive science. Behavioral and Brain Sciences 36:181-253. doi: 10.1017/ S0140525X12000477. [FF-S]

Clayton, E. C., Rajkowski, J., Cohen, J. D. \& Aston-Jones, G. (2004) Phasic activation of monkey locus ceruleus neurons by simple decisions in a forced-choice task. The Journal of Neuroscience 24(44):9914-20. Available at: http://doi.org/10. 1523/JNEUROSCI.2446-04.2004. [SB]

Clelland, C. D., Choi, M., Romberg, C., Clemenson, G. D., Jr., Fragniere, A., Tyers, P., Jessberger, S., Saksida, L. M., Barker, R. A., Gage, F. H. \& Bussey, T. J. 
(2009) A functional role for adult hippocampal neurogenesis in spatial pattern separation. Science 325: 210-13. [CH]

Clewett, D., Schoeke, A. \& Mather, M. (2014) Locus coeruleus neuromodulation of memories encoded during negative or unexpected action outcomes. Neurobiology of Learning and Memory 111:65-70. [aMM]

Clore, G. L. \& Huntsinger, J. R. (2007) How emotions inform judgment and regulate thought. Trends in Cognitive Sciences 11(9):393-99. [JRH]

Cocchi, L., Zalesky, A., Fornito, A. \& Mattingley, J. B. (2013) Dynamic cooperation and competition between brain systems during cognitive control. Trends in Cognitive Sciences 17(10):493-501. [aMM]

Cohen, J. D., Servan-Schreiber, D. \& McClelland, J. L. (1992) A parallel distributed processing approach to automaticity. The American Journal of Psychology 105:239-69. doi: 10.2307/1423029. [CMW]

Constantinople, C. M. \& Bruno, R. M. (2011) Effects and mechanisms of wakefulness on local cortical networks. Neuron 69(6):1061-68. Available at: http://dx. doi.org/10.1016/j.neuron.2011.02.040. [aMM, AB]

Corbetta, M., Patel, G. \& Shulman, G. L. (2008) The reorienting system of the human brain: From environment to theory of mind. Neuron 58(3):306-24. [aMM, SJS]

Correa, A., Cappucci, P., Nobre, A. C. \& Lupiáñez, J. (2010) The two sides of temporal orienting: Facilitating perceptual selection, disrupting response selection. Experimental Psychology 57:142-48. doi: 10.1027/1618-3169/ a000018. [CMW]

Cousijn, H., Rijpkema, M., Qin, S., van Marle, H. J., Franke, B., Hermans, E. J., van Wingen, G. \& Fernández, G. (2010) Acute stress modulates genotype effects on amygdala processing in humans. Proceedings of the National Academy of Sciences of the United States of America (21):9867-72. [aMM]

Cox, D. J., Racca, C. \& Lebeau, F. E. N. (2008) $\beta$-Adrenergic receptors are differentially expressed in distinct interneuron subtypes in the rat hippocampus. The Journal of Comparative Neurology 509(6):551-65. doi: 10.1002/cne.21758. [aMM]

Craig, A. D. (2009) How do you feel - now? The anterior insula and human awareness. Nature Reviews Neuroscience 10(1):59-70. doi: 10.1038/nrn2555. [aMM]

Craik, F. I. \& Lockhart, R. S. (1972) Levels of processing: A framework for memory research. Journal of Verbal Learning and Verbal Behavior 11(6):671-84. [JRH]

Critchley, H. D., Tang, J., Glaser, D., Butterworth, B. \& Dolan, R. J. (2005) Anterior cingulate activity during error and autonomic response. NeuroImage 27(4):88595. [aMM]

Crockett, M. J., Clark, L. \& Robbins, T. W. (2009) Reconciling the role of serotonin in behavioral inhibition and aversion: Acute tryptophan depletion abolishes punishment-induced inhibition in humans. The Journal of Neuroscience 29 (38):11993-99. [HM]

Cunningham, W. A. \& Brosch, T. (2012) Motivational salience: Amygdala tuning from traits, needs, values, and goals. Current Directions in Psychological Science 21:54-59. doi: 10.1177/0963721411430832. [AM]

Dahl, D. \& Winson, J. (1985) Action of norepinephrine in the dentate gyrus. I. Stimulation of locus coeruleus. Experimental Brain Research 59:491-96. $[\mathrm{CH}]$

Dalley, J. W., McGaughy, J., O’Connell, M. T., Cardinal, R. N., Levita, L. \& Robbins, T. W. (2001) Distinct changes in cortical acetylcholine and noradrenaline efflux during contingent and noncontingent performance of a visual attentional task. The Journal of Neuroscience 21(13):4908-14. [SB]

Damasio, A. R., Everitt, B. \& Bishop, D. (1996) The somatic marker hypothesis and the possible functions of the prefrontal cortex [and discussion]. Philosophical Transactions of the Royal Society B: Biological Sciences 351(1346):1413-20. [HM]

Dang-Vu, T. T., Schabus, M., Desseilles, M., Albouy, G., Boly, M., Darsaud, A., Gais, S., Rauchs, G., Sterpenich, V. \& Vandewalle, G. (2008) Spontaneous neural activity during human slow wave sleep. Proceedings of the National Academy of Sciences of the United States of America 105(39):15160-65. [aMM]

Dani, J. A. \& Bertrand, D. (2007) Nicotinic acetylcholine receptors and nicotinic cholinergic mechanisms of the central nervous system. Annual Review of Pharmacology and Toxicology 47:699-729. doi: 10.1146/annurev.pharmtox.47.120505.105214. $\quad[\mathrm{AB}]$

Datta, S. (2010) Cellular and chemical neuroscience of mammalian sleep. Sleep Medicine 11:431-40. doi: 10.1016/j.sleep.2010.02.002. [AB]

Dayan, P. \& Yu, A. J. (2006) Phasic norepinephrine: A neural interrupt signal for unexpected events. Network: Computation in Neural Systems 17:335-50. doi 10.1080/09548980601004024. [FF-S]

De Carvalho Myskiw, J., Furini, C. R. G., Benetti, F. \& Izquierdo, I. (2014) Hippocampal molecular mechanisms involved in the enhancement of fear extinction caused by exposure to novelty. Proceedings of the National Academy of Sciences of the United States of America 111(12):4572-77. [MR]

de Kloet, E. R., Joels, M. \& Holsboer, F. (2005) Stress and the brain: From adaptation to disease. Nature Reviews Neuroscience 6(6):463-75. Available at: http:// www.ncbi.nlm.nih.gov/pubmed/15891777. [RH]
De Martino, B., Strange, B. A. \& Dolan, R. J. (2008) Noradrenergic neuromodulation of human attention for emotional and neutral stimuli. Psychopharmacology (Berlin) 197:127-36. [HDC, RMT]

de Quervain, D. J., Kolassa, I.-T., Ertl, V., Onyut, P. L., Neuner, F., Elbert, T. \& Papassotiropoulos, A. (2007) A deletion variant of the $\alpha_{2 b}$-adrenoceptor is related to emotional memory in Europeans and Africans. Nature Neuroscience 10(9):1137-39. [aMM, RMT]

de Quervain, D. J., Roozendaal, B., Nitsch, R. M., McGaugh, J. L. \& Hock, C. (2000) Acute cortisone administration impairs retrieval of long-term declarative memory in humans. Nature Neuroscience 3(4):313-14. Available at: http://www. ncbi.nlm.nih.gov/pubmed/10725918. [RH]

De Vos, H., Vauquelin, G., Keyser, J., Backer, J. P. \& Liefde, I. (1992) Regional distribution of $\alpha_{2 \mathrm{~A}}$-and $\alpha_{2 \mathrm{~B}}$-Adrenoceptor subtypes in postmortem human brain. Journal of Neurochemistry 58(4):1555-60. [aMM]

Decamp, E., Clark, K. \& Schneider, J. S. (2011) Effects of the alpha-2 adrenoceptor agonist guanfacine on attention and working memory in aged non-human primates. European Journal of Neuroscience 34(6):1018-22. [aMM]

Delaney, A. J., Crane, J. W. \& Sah, P. (2007) Noradrenaline modulates transmission at a central synapse by a presynaptic mechanism. Neuron 56(5):880-92. Available at: http://dx.doi.org/10.1016/j.neuron.2007.10.022. [aMM]

Denny, B. T., Inhoff, M. C., Zerubavel, N., Davachi, L. \& Ochsner, K. N. (2015) Getting over it: Long-lasting effects of emotion regulation on amygdala response. Psychological Science 26(9):1377-88. [TDH]

Descarries, L., Watkins, K. C. \& Lapierre, Y. (1977) Noradrenergic axon terminals in the cerebral cortex of rat: III. Topometric ultrastructural analysis. Brain Research 133(2):197-222. [aMM]

Desimone, R. \& Duncan, J. (1995) Neural mechanisms of selective visual attention. Annual Review of Neuroscience 18:193-222. [aMM, RHP]

Devauges, V. \& Sara, S. J. (1990) Activation of the noradrenergic system facilitates an attentional shift in the rat. Behavioural Brain Research 39(1):19-28. [SB]

Devauges, V. \& Sara, S. J. (1991) Memory retrieval enhancement by locus coeruleus stimulation: Evidence for mediation by $\beta$-receptors. Behavioural Brain Research 43(1):93-97. [aMM]

Devilbiss, D. M. \& Waterhouse, B. D. (2000) Norepinephrine exhibits two distinct profiles of action on sensory cortical neuron responses to excitatory synaptic stimuli. Synapse 37(4):273-82. [EE, RN, rMM]

Devilbiss, D. M. \& Waterhouse, B. D. (2004) The effects of tonic locus ceruleus output on sensory-evoked responses of ventral posterior medial thalamic and barrel field cortical neurons in the awake rat. Journal of Neuroscience 24:1077385. $[\mathrm{QG}]$

Devilbiss, D. M. \& Waterhouse, B. D. (2011) Phasic and tonic patterns of locus coeruleus output differentially modulate sensory network function in the awake rat. Journal of Neurophysiology 105(1):69-87. doi: 10.1152/jn.00445.2010. [aMM]

Devilbiss, D. M., Waterhouse, B. D., Berridge, C. W. \& Valentino, R. (2012) Corticotropin-releasing factor acting at the locus coeruleus disrupts thalamic and cortical sensory-evoked responses. Neuropsychopharmacology 37(9):202030. $[\mathrm{aMM}]$

Diamond, D. M., Park, C. R., Campbell, A. M. \& Woodson, J. C. (2005) Competitive interactions between endogenous LTD and LTP in the hippocampus underlie the storage of emotional memories and stress-induced amnesia. Hippocampus 15(8):1006-25. [aMM]

Diamond, D. M., Park, C. R. \& Woodson, J. C. (2004) Stress generates emotional memories and retrograde amnesia by inducing an endogenous form of hippocampal LTP. Hippocampus 14(3):281-91. [BAS]

Diekelmann, S. \& Born, J. (2010) The memory function of sleep. Nature Reviews Neuroscience 11(2):114-26. [aMM]

Ding, F., O'Donnell, J., Thrane, A. S., Zeppenfeld, D., Kang, H., Xie, L., Wang, F. \& Nedergaard, M. (2013) $\alpha_{1}$-Adrenergic receptors mediate coordinated $\mathrm{Ca}^{2+}$ signaling of cortical astrocytes in awake, behaving mice. Cell Calcium 54(6):387-94. [rMM]

Dolcos, F., Diaz-Granados, P., Wang, L. H. \& McCarthy, G. (2008) Opposing influences of emotional and non-emotional distracters upon sustained prefrontal cortex activity during a delayed-response working memory task. Neuropsychologia 46(1):326-35. doi: 10.1016/j.neuropsychologia.2007.07.010 [aMM]

Dolcos, F., Iordan, A. D. \& Dolcos, S. (2011) Neural correlates of emotion-cognition interactions: A review of evidence from brain imaging investigations. Journal of Cognitive Psychology 23:669-94. [aMM]

Dolcos, F., LaBar, K. S. \& Cabeza, R. (2004) Interaction between the amygdala and the medial temporal lobe memory system predicts better memory for emotional events. Neuron 42(5):855-63. [aMM, BR]

Dolcos, F. \& McCarthy, G. (2006) Brain systems mediating cognitive interference by emotional distraction. Journal of Neuroscience 26(7):2072-79. doi: 10.1523/ JNEUROSCI.5042-05.2006. [aMM]

Donchin, E. \& Coles, M. G. (1988) Is the P300 component a manifestation of context updating? Behavioral and Brain Sciences 11:357-427. [DT] 
Donoso, M., Collins, A. G. E. \& Koechlin, E. (2014) Foundations of human reasoning in the prefrontal cortex. Science 344(6191):1481-86. [TDH]

Dreisbach, G. \& Fischer, R. (2012) Conflicts as aversive signals. Brain and Cognition 78:94-98. [RHP]

Dudek, S. M. \& Bear, M. F. (1992) Homosynaptic long-term depression in area CA1 of hippocampus and effects of N-methyl-D-aspartate receptor blockade. Proceedings of the National Academy of Sciences of the United States of America 89(10):4363-67. [BAS]

Duman, R. S. \& Enna, S. (1986) A procedure for measuring $\alpha_{2}$-adrenergic receptormediated inhibition of cyclic AMP accumulation in rat brain slices. Brain Research 384(2):391-94. [aMM]

Duncan, J. (1996) Cooperating brain systems in selective perception and action. In: Attention and Performance XVI, ed. T. Inui \& J. L. McClelland, pp. 549-78. MIT Press. [RHP]

Duncan, J. (2006) EPS Mid-Career Award 2004 - Brain mechanisms of attention. Quarterly Journal of Experimental Psychology 59(1):2-27. doi: 10.1080/ 17470210500260674. [aMM]

Dunsmoor, J. E., Murty, V. P., Davachi, L. \& Phelps, E. A. (2015) Emotional learning selectively and retroactively strengthens memories for related events. Nature 520(7547): 345-48. [arMM, MR]

Dutton, D. G. \& Aron, A. P. (1974) Some evidence for heightened sexual attraction under conditions of high anxiety. Journal of Personality and Social Psychology 30(4):510-17. [JRH, rMM]

Duval, E. R., Moser, J. S., Huppert, J. D. \& Simons, R. F. (2013) What's in a face? The late positive potential reflects the level of facial affect expression. Journal of Psychophysiology 27:27-38. [KK]

Easterbrook, J. A. (1959) The effect of emotion on cue utilization and the organization of behavior. Psychological Review 66(3):183-201. doi: 10.1037/ h0047707. [aMM]

Easterbrook, J. A. (1982) This week's citation classic. Current Contents (12):20. [aMM]

Eckert, M. A., Menon, V., Walczak, A., Ahlstrom, J., Denslow, S., Horwitz, A. \& Dubno, J. R. (2009) At the heart of the ventral attention system: The right anterior insula. Human Brain Mapping 30(8):2530-41. doi: 10.1002/ hbm.20688. [aMM]

Eckhoff, P., Wong-Lin, K. F. \& Holmes, P. (2009) Optimality and robustness of a biophysical decision-making model under norepinephrine modulation. Journal of Neuroscience 29(13):4301-11. [CMW]

Edeline, J.-M. (1995) The $\alpha_{2}$-adrenergic antagonist idazoxan enhances the frequency selectivity and increases the threshold of auditory cortex neurons. Experimental Brain Research 107:221-40. [QG]

Edeline, J.-M (2012) Beyond traditional approaches to understanding the functional role of neuromodulators in sensory cortices. Frontiers in Behavioral Neuroscience 6:45. [QG]

Edeline, J.-M., Manunta, Y. \& Hennevin, E. (2011) Induction of selective plasticity in the frequency tuning of auditory cortex and auditory thalamus neurons by locus coeruleus stimulation. Hearing Research 274:75-84. [QG]

Edmiston, E. K., McHugo, M., Dukic, M. S., Smith, S. D., Abou-Khalil, B., Eggers, E. \& Zald, D. H. (2013) Enhanced visual cortical activation for emotional stimuli is preserved in patients with unilateral amygdala resection. The Journal of Neuroscience 33(27):11023-31. [rMM]

Egli, R. E., Kash, T. L., Choo, K., Savchenko, V., Matthews, R. T., Blakely, R. D. \& Winder, D. G. (2005) Norepinephrine modulates glutamatergic transmission in the bed nucleus of the stria terminalis. Neuropsychopharmacology 30(4):65768. [aMM]

Ego-Stengel, V., Bringuier V. \& Shulz D. E. (2002) Noradrenergic modulation of functional selectivity in the cat visual cortex: An in vivo extracellular and intracellular study. Neuroscience 111(2):275-89. [QG]

Ehlers, M. E., Palombo, D. J., Mueller, D., Levine, B., Anderson, A. K. \& Todd, R. M. (2015) Grey matter differences are predicted by variation in the ADRA2b gene. Poster presented at the Annual Meeting of the Society for Neuroscience, Chicago, IL, USA. [RMT]

Ehrlich, I., Humeau, Y., Grenier, F., Ciocchi, S., Herry, C. \& Luthi, A. (2009) Amygdala inhibitory circuits and the control of fear memory. Neuron 62(6):75771. Available at: http://www.ncbi.nlm.nih.gov/pubmed/19555645. [RH]

Einhäuser, W., Stout, J., Koch, C. \& Carter, O. L. (2008) Pupil dilation reflects perceptual selection and predicts subsequent stability in perceptual rivalry. Proceedings of the National Academy of Sciences of the United States of America 105(5):1704-709. Available at: http://doi.org/10.1073/pnas.0707727105. [SB]

Ekman, P. (1993) Facial expression and emotion. The American Psychologist 48:384-92. [TDH]

Elam, M., Thorén, P. \& Svensson, T. H. (1986) Locus coeruleus neurons and sympathetic nerves: Activation by visceral afferents. Brain Research 375(1):11725. [HDC, rMM]

Elam, M., Yoa, T., Svensson, T. \& Thoren, P. (1984) Regulation of locus coeruleus neurons and splanchnic, sympathetic nerves by cardiovascular afferents. Brain Research 290(2):281-87. [HDC, rMM]
Eldar, E. (2014) Focus versus breadth: The effects of neural gain on information processing. Doctoral dissertation, Princeton University. [EE]

Eldar, E., Cohen, J. D. \& Niv, Y. (2013) The effects of neural gain on attention and learning. Nature Neuroscience 16(8):1146-53. [aMM, EE, CMW]

Eldar, E., Niv, Y. \& Cohen, J. D. (in press) Do you see the forest or the tree? Neural gain and breadth versus focus in perceptual processing. Psychological Science. $[\mathrm{EE}]$

Elliot, A. J. \& Covington, M. V. (2001) Approach and avoidance motivation. Educational Psychology Review 13(2):73-92. [HM]

Eppinger, B., Hämmerer, D. \& Li, S.-C. (2011) Neuromodulation of reward-based learning and decision making in human aging. Annals of the New York Academy of Sciences 1235:1-17. [MRN]

Eppinger, B., Kray, J., Mock, B. \& Mecklinger, A. (2008) Better or worse than expected? Aging, learning, and the ERN. Neuropsychologia 46:521-39. [MRN]

Eschenko, O., Magri, C., Panzeri, S. \& Sara, S. J. (2012) Noradrenergic neurons of the locus coeruleus are phase locked to cortical up-down states during sleep. Cerebral Cortex 22(2):426-35. [aMM]

Eschenko, O. \& Sara, S. J. (2008) Learning-dependent, transient increase of activity in noradrenergic neurons of locus coeruleus during slow wave sleep in the rat: Brain stem-cortex interplay for memory consolidation? Cerebral Cortex 18 (11):2596-603. doi: 10.1093/cercor/bhn020. [aMM]

Eysenck, M. W., Derakshan, N., Santos, R. \& Calvo, M. G. (2007) Anxiety and cognitive performance: Attentional control theory. Emotion 7(2):336. [aMM]

Facchinetti, L. D., Imbiriba, L. A., Azevedo, T. M., Vargas, C. D. \& Volchan, E. (2006) Postural modulation induced by pictures depicting prosocial or dangerous contexts. Neuroscience Letters 410(1):52-56. [HM]

Fallon, J. H., Koziell, D. A. \& Moore, R. Y. (1978) Catecholamine innervation of the basal forebrain: II. Amygdala, suprarhinal cortex and entorhinal cortex. The Journal of Comparative Neurology 180(3):509-31. doi: 10.1002/ cne.901800308. [aMM]

Fan, J., Gu, X., Guise, K. G., Liu, X., Fossella, J., Wang, H. \& Posner, M. I. (2009) Testing the behavioral interaction and integration of attentional networks. Brain and Cognition 70: 209-20. doi: 10.1016/j.bandc.2009.02.002. [CMW]

Fan, J., McCandliss, B. D., Sommer, T., Raz, A. \& Posner, M. I. (2002) Testing the efficiency and independence of attentional networks. Journal of Cognitive Neuroscience 14(3):340—47. [CMW]

Fastenrath, M., Coynel, D., Spalek, K., Spalek, K., Milnik, A., Gschwind, L., Roozendaal, B., Papassotiropoulos, A. \& de Quervain, J. F. (2014) Dynamic modulation of amygdala-hippocampal connectivity by emotional arousal. The Journal of Neuroscience 34(42):13935-47. [BR]

Fazendeiro, T., Chenier, T. \& Winkielman, P. (2007) How dynamics of thinking create affective and cognitive feelings: Psychology and neuroscience of the connection between fluency, liking, and memory. In: Social neuroscience, ed. E. Harmon-Jones \& P. Winkielman, pp. 271-89. Guilford Press. [RHP]

Fecteau, J. H. \& Munoz, D. P. (2006) Salience, relevance, and firing: A priority map for target selection. Trends in Cognitive Sciences 10(8):382-90. doi: 10.1016/j. tics.2006.06.011. [aMM]

Feinstein, J. S., Adolphs, R., Damasio, A. \& Tranel, D. (2011) The human amygdala and the induction and experience of fear. Current Biology 21(1):34-38. [rMM]

Feinstein, J. S., Buzza, C., Hurlemann, R., Follmer, R. L., Dahdaleh, N. S., Coryell, W. H., Welsh, M. J., Tranel, D. \& Wemmie, J. A. (2013) Fear and panic in humans with bilateral amygdala damage. Nature Neuroscience 16(3):270-72. [rMM]

Feldman, L. A. (1995) Valence focus and arousal focus: Individual differences in the structure of affective experience. Journal of Personality and Social Psychology 69:153-66. doi: 10.1037/0022-3514.69.1.153.search. [AM]

Ferrero, J. J., Alvarez, A. M., Ramirez-Franco, J., Godino, M. C., Bartolome-Martin, D., Aguado, C., Torres, M., Lujan, R., Ciruela, F. \& Sanchez-Prieto, J. (2013) $\beta$ Adrenergic receptors activate Epac, translocate Munc13-1 and enhance the Rab3A-Riml $\alpha$ interaction to potentiate glutamate release at cerebrocortical nerve terminals. Journal of Biological Chemistry 288(43):31370-85. [aMM]
[ä.

Ferry, B., Roozendaal, B. \& McGaugh, J. L. (1999a) Basolateral amygdala noradrenergic influences on memory storage are mediated by an interaction between beta- and alpha-1-adrenoceptors. The Journal of Neuroscience 19:5119-23. [CGA]

Ferry, B., Roozendaal, B. \& McGaugh, J. L. (1999b) Involvement of alpha-1-adrenoceptors in the basolateral amygdala in modulation of memory storage. European Journal of Pharmacology 372:9-16. [CGA]

Fink, K., Göthert, M., Molderings, G. \& Schlicker, E. (1989) N-Methyl-D-aspartate (NMDA) receptor-mediated stimulation of noradrenaline release, but not release of other neurotransmitters, in the rat brain cortex: Receptor location, characterization and desensitization. Naunyn-Schmiedeberg's Archives of Pharmacology 339(5):514-21. [aMM]

Fink, K., Schultheiß, R. \& Göthert, M. (1992) Stimulation of noradrenaline release in human cerebral cortex mediated by $N$-methyl-D-aspartate (NMDA) and nonNMDA receptors. British Journal of Pharmacology 106(1):67-72. [aMM] 
Fischer, J. \& Whitney, D. (2012) Attention gates visual coding in the human pulvinar. Nature Communications 3:1051. [aMM]

Fischer, R., Plessow, F. \& Kiesel, A. (2010) Auditory warning signals affect mechanisms of response selection: Evidence from a Simon task. Experimental Psychology 57:89-97. doi: 10.1027/1618-3169/a000012. [CMW]

Florin-Lechner, S. M., Druhan, J. P., Aston-Jones, G. \& Valentino, R. J. (1996) Enhanced norepinephrine release in prefrontal cortex with burst stimulation of the locus coeruleus. Brain Research 742(1/2):89-97. [aMM, RN]

Foote, S. L., Aston-Jones, G. \& Bloom, F. E. (1980) Impulse activity of locus coeruleus neurons in awake rats and monkeys is a function of sensory stimulation and arousal. Proceedings of the National Academy of Sciences of the United States of America 77(5):3033-37. [aMM, SB]

Foote, S. L., Freedman, R. \& Oliver, A. P. (1975) Effects of putative neurotransmitters on neuronal activity in monkey auditory cortex. Brain Research 86 (2):229-42. [aMM, QG]

Foote, S. L. \& Morrison, J. H. (1987) Extrathalamic modulation of cortical function. Annual Review of Neuroscience 10:67-95. doi: 10.1146/annurev. neuro.10.1.67. [aMM]

Frankland, P. W. \& Josselyn, S. A. (2015) Memory allocation. Neuropsychopharmacology 40(1):243-43. [aMM]

Freedman, R., Hoffer, B. J., Woodward, D. J. \& Puro, D. (1977) Interaction of norepinephrine with cerebellar activity evoked by mossy and climbing fibers. Experimental Neurology 55(1):269-88. [aMM]

Frey, S., Bergado-Rosado, J., Seidenbecher, T., Pape, H.-C. \& Frey, J. U. (2001) Reinforcement of early long-term potentiation (early-LTP) in dentate gyrus by stimulation of the basolateral amygdala: Heterosynaptic induction mechanisms of late-LTP. The Journal of Neuroscience 21(10):3697-703. [aMM]

Frey, U. \& Morris, R. G. (1997) Synaptic tagging and long-term potentiation. Nature $385(6616): 533-36 . \quad[\mathrm{MR}]$

Frey, U. \& Morris, R. G. (1998) Synaptic tagging: Implications for late maintenance of hippocampal long-term potentiation. Trends in Neurosciences 21(5):18188. $[\mathrm{MR}]$

Fries, P. (2009) Neuronal gamma-band synchronization as a fundamental process in cortical computation. Annual Review of Neuroscience 32(1):209-24. doi: 10.1146/annurev.neuro.051508.135603. [aMM]

Fries, P., Reynolds, J. H., Rorie, A. E. \& Desimone, R. (2001) Modulation of oscillatory neuronal synchronization by selective visual attention. Science 291 (5508):1560-63. doi: 10.2307/3082512. [aMM]

Frijda, N. H. (1986) The emotions. Cambridge University Press. [AM]

Friston, K. (2005) A theory of cortical responses. Philosophical Transactions of the Royal Society B: Biological Sciences 360:815-36. doi: 10.1098/ rstb.2005.1622. [FF-S]

Friston, K. (2010) The free-energy principle: A unified brain theory? Nature Reviews Neuroscience 11(2):127-38. [aMM, FF-S]

Fuster, J. M. (1991) The prefrontal cortex and its relation to behavior. Progress in Brain Research 87:201-11. [SJS]

Gais, S., Rasch, B., Dahmen, J. C., Sara, S. \& Born, J. (2011) The memory function of noradrenergic activity in non-REM sleep. Journal of Cognitive Neuroscience 23 (9):2582-92. doi: 10.1162/jocn.2011.21622. [aMM]

Gambino, F., Pagès, S., Kehayas, V., Baptista, D., Tatti, R., Carleton, A. \& Holtmaat, A. (2014) Sensory-evoked LTP driven by dendritic plateau potentials in vivo. Nature 515(7525):116-19. [MEL]

Gardner, J. M. \& Karmel, B. Z. (1983) Attention and arousal in preterm and fullterm neonates. Infants born at risk: Behavior and development, ed. T. Field \& A. Sostek, pp. 69-98. Grune \& Stratton. [RG]

Gardner, J. M. \& Karmel, B. Z. (1984) Arousal effects on visual preferences in neonates. Developmental Psychology 20(3):374-77. doi: 10.1037/00121649.20.3.374. [RG]

Gardner-Medwin, A. (1976) The recall of events through the learning of associations between their parts. Proceedings of the Royal Society of London B 194:375402. [CH]

Garfinkel, S. N., Barrett, A. B., Minati, L., Dolan, R. J., Seth, A. K. \& Critchley, H. D. (2013). What the heart forgets: Cardiac timing influences memory for words and is modulated by metacognition and interoceptive sensitivity. Psychophysiology 50(6):505-12. doi: 10.1111/psyp.12039. [HDC, rMM]

Garfinkel, S. N., Minati, L., Gray, M. A., Seth, A. K., Dolan, R. J. \& Critchley, H. D. (2014) Fear from the heart: Sensitivity to fear stimuli depends on individual heartbeats. The Journal of Neuroscience 34(19):6573-82. [HDC, rMM]

Garrett, D. D., Samanez-Larkin, G. R., MacDonald, S. W. S., Lindenberger, U., Mcintosh, A. R. \& Grady, C. L. (2013) Moment-to-moment brain signal variability: A next frontier in human brain mapping? Neuroscience and Biobehavioral Reviews 37:610-24. [MRN]

Gaspar, P., Berger, B., Febvret, A., Vigny, A. \& Henry, J. P. (1989) Catecholamine innervation of the human cerebral cortex as revealed by comparative immunohistochemistry of tyrosine hydroxylase and dopamine-beta-hydroxylase. Journal of Comparative Neurology 279(2):249-71. [aMM]
Gaucher, Q. \& Edeline, J. M. (2015) Stimulus-specific effects of noradrenaline in auditory cortex: Implications for the discrimination of communication sounds. The Journal of Physiology 593(4):1003-20. [QG, rMM]

Gehring, W. J. \& Willoughby, A. R. (2002) The medial frontal cortex and the rapid processing of monetary gains and losses. Science 295(5563):2279-82. doi: 10.2307/3076349. [aMM]

Gelinas, J. N. \& Nguyen, P. V. (2005) Beta-adrenergic receptor activation facilitates induction of a protein synthesis-dependent late phase of long-term potentiation. Journal of Neuroscience 25(13):3294-303. doi: 10.1523/jneurosci.417504.2005. [aMM]

Gelinas, J. N., Tenorio, G., Lemon, N., Abel, T. \& Nguyen, P. V. (2008) ß-Adrenergic receptor activation during distinct patterns of stimulation critically modulates the PKA-dependence of LTP in the mouse hippocampus. Learning and Memory 15(5):281-89. [aMM]

Gereau, R. \& Conn, P. J. (1994) A cyclic AMP-dependent form of associative synaptic plasticity induced by coactivation of beta-adrenergic receptors and metabotropic glutamate receptors in rat hippocampus. The Journal of Neuroscience 14(5):3310-18. [aMM]

Geva, R., Eshel, R., Leitner, Y., Valevski, A. F. \& Harel, S. (2006) Neuropsychological outcome of children with intrauterine growth restriction: A 9-year prospective study. Pediatrics 118(1):91-100. doi: 10.1542/peds.2005-2343. [RG]

Geva, R. \& Feldman, R. (2008) A neurobiological model for the effects of early brainstem functioning on the development of behavior and emotion regulation in infants: Implications for prenatal and perinatal risk. Journal of Child Psychology and Psychiatry 49(10):1031-41. doi: 10.1111/j.1469-7610.2008.01918.x. [RG]

Geva, R., Gardner, J. M. \& Karmel, B. Z. (1999) Feeding-based arousal effects on visual recognition memory in early infancy. Developmental Psychology $35(3): 640-50 . \quad[R G]$

Geva, R., Yaron, H. \& Kuint, J. (2013) Neonatal sleep predicts attention orienting and distractibility. Journal of Attention Disorders 20(2):138-50. [RG]

Ghersi, C., Bonfanti, A., Manzari, B., Feligioni, M., Raiteri, M. \& Pittaluga, A. (2003) Pharmacological heterogeneity of release-regulating presynaptic AMPA/kainate receptors in the rat brain: Study with receptor antagonists. Neurochemistry International 42:283-92. doi: 10.1016/S0197-0186(02)00129-8. [AB]

Ghosh, S. \& Chattarji, S. (2015) Neuronal encoding of the switch from specific to generalized fear. Nature Neuroscience 18(1):112-20. [BR]

Gilbert, P. E., Kesner, R. P. \& Lee, I. (2001) Dissociating hippocampal subregions: A double dissociation between dentate gyrus and CA1. Hippocampus 11:62636. $[\mathrm{CH}]$

Gilsbach, R. \& Hein, L. (2008) Presynaptic metabotropic receptors for acetylcholine and adrenaline/noradrenaline. In: Pharmacology of neurotransmitter release, ed. T. C. Südhof \& K. Starke, pp. 261-88. Springer. [aMM]

Gilzenrat, M. S., Holmes, B. D., Rajkowski, J., Aston-Jones, G. \& Cohen, J. D. (2002) Simplified dynamics in a model of noradrenergic modulation of cognitive performance. Neural Networks 15:647-63. [CMW]

Gire, D. H. \& Schoppa, N. E. (2008) Long-term enhancement of synchronized oscillations by adrenergic receptor activation in the olfactory bulb. Journal of Neurophysiology 99(4):2021-25. doi: 10.1152/jn.01324.2007. [aMM]

Goldman-Rakic, P. S. (1990) Cellular and circuit basis of working memory in prefrontal cortex of nonhuman primates. Progress in Brain Research 85:325-35. [SJS]

Gonzales, R. A., Brown, L. M., Jones, T. W., Trent, R. D., Westbrook, S. L. \& Leslie, S. W. (1991) N-Methyl-D-aspartate mediated responses decrease with age in Fischer 344 rat brain. Neurobiology of Aging 12(3):219-25. [aMM]

Goolsby, B. A., Shapiro, K. L., Silvert, L., Kiss, M., Fragopanagos, N., Taylor, J. G., Eimer, M., Nobre, A. C. \& Raymond, J. E. (2009) Feature-based inhibition underlies the affective consequences of attention. Visual Cognition 17:50030. $[$ RHP $]$

Goossens, L., Kukolja, J., Onur, O. A., Fink, G. R., Maier, W., Griez, E., Schruers, K. \& Hurlemann, R. (2009) Selective processing of social stimuli in the superficial amygdala. Human Brain Mapping 30(10):3332-38. Available at: http://www. ncbi.nlm.nih.gov/pubmed/19347877. [RH]

Göthert, M. \& Fink, K. (1991) Stimulation of noradrenaline release in the cerebral cortex via presynaptic $N$-Methyl-D-aspartate (NMDA) receptors and their pharmacological characterization. In: Recent advances in neuropharmacology, ed. H. Bönisch, K.-H. Graefe, S. Z. Langer \& E. Schömig, pp. 121-27. Springer. [aMM]

Grace, A. A., Floresco, S. B., Goto, Y. \& Lodge, D. J. (2007) Regulation of firing of dopaminergic neurons and control of goal-directed behaviors. Trends in Neurosciences 30(5):220-27. [MR]

Grant, S. J., Aston-Jones, G. \& Redmond, D. E., Jr. (1988) Responses of primate locus coeruleus neurons to simple and complex sensory stimuli. Brain Research Bulletin 21(3):401-10. [aMM]

Gratton, G., Coles, M. G. H., Sirevaag, E. J., Eriksen, C. W. \& Donchin, E. (1988) Pre- and post-stimulus activation of response channels: A psychophysiological analysis. Journal of Experimental Psychology: Human Perception and Performance 14:331-44. doi: 10.1037/0096-1523.14.3.331. [CMW] 
Gray, J. \& Ball, G. (1970) Frequency-specific relation between hippocampal theta rhythm, behavior, and amobarbital action. Science 168(3936):1246-48. [rMM]

Gray, J., McNaughton, N., James, D. \& Kelly, P. (1975) Effect of minor tranquillisers on hippocampal $\theta$ rhythm mimicked by depletion of forebrain noradrenaline. Nature 258:424-25. [rMM]

Gray, M. A., Rylander, K., Harrison, N. A., Wallin, B. G. \& Critchley. H. D. (2009) Following one's heart: Cardiac rhythms gate central initiation of sympathetic reflexes. The Journal of Neuroscience 29:1817-25. [HDC]

Gregory, R. L. (1970) The intelligent eye. Weidenfeld and Nicolson. [C-CC]

Grienberger, C., Chen, X. \& Konnerth, A. (2014) NMDA receptor-dependent multidendrite $\mathrm{Ca}^{2+}$ spikes required for hippocampal burst firing in vivo. Neuron 81(6):1274-81. [MEL]

Grilli, M., Zappettini, S., Zanardi, A., Lagomarsino, F., Pittaluga, A., Zoli, M. \& Marchi, M. (2009) Exposure to an enriched environment selectively increases the functional response of the pre-synaptic NMDA receptors which modulate noradrenaline release in mouse hippocampus. Journal of Neurochemistry 110 (5):1598-606. doi: 10.1111/j.1471-4159.2009.06265.x. [aMM]

Groch, S., Wilhelm, I., Diekelmann, S., Sayk, F., Gais, S. \& Born, J. (2011) Contribution of norepinephrine to emotional memory consolidation during sleep. Psychoneuroendocrinology 36(9):1342-50. [aMM]

Gross, J. J. (2015) Emotion regulation: Current status and future prospects. Psychological Inquiry 26(1):1-26. [TDH]

Gross, J. J. \& Feldman Barrett, L. (2011) Emotion generation and emotion regulation: One or two depends on your point of view. Emotion Review 3(1):8-16. [TDH]

Grudzien, A., Shaw, P., Weintraub, S., Bigio, E., Mash, D. C. \& Mesulam, M. M. (2007) Locus coeruleus neurofibrillary degeneration in aging, mild cognitive impairment and early Alzheimer's disease. Neurobiology of Aging 28:327-35. [MRN]

Grühn, D. \& Scheibe, S. (2008) Age-related differences in valence and arousal ratings of pictures from the International Affective Picture System (IAPS): Do ratings become more extreme with age? Behavior Research Methods 40(2):51221. [rMM]

Guitart-Masip, M., Duzel, E., Dolan, R. \& Dayan, P. (2014) Action versus valence in decision making. Trends in Cognitive Sciences 18(4):194-202. [HM]

Guitart-Masip, M., Fuentemilla, L., Bach, D. R., Huys, Q. J., Dayan, P., Dolan, R. J. \& Duzel, E. (2011) Action dominates valence in anticipatory representations in the human striatum and dopaminergic midbrain. The Journal of Neuroscience 31(21):7867-75. [HM]

Guitart-Masip, M., Huys, Q. J., Fuentemilla, L., Dayan, P., Duzel, E. \& Dolan, R. J. (2012) Go and no-go learning in reward and punishment: Interactions between affect and effect. NeuroImage 62(1):154-66. [HM]

Gulledge, A. T., Bucci, D. J., Zhang, S. S., Matsui, M. \& Yeh, H. H. (2009) M1 receptors mediate cholinergic modulation of excitability in neocortical pyramidal neurons. Journal of Neuroscience 29:9888-902. doi: 10.1523/JNEUROSCI.1366-09.2009. [AB]

Gurfinkel, E. (1973) Physical foundations of stabilography. Agressologie: Revue internationale de physio-biologie et de pharmacologie appliquees aux effets de l'agression 14(Spec No C):9-13. [HM]

Hagenaars, M. A., Oitzl, M. \& Roelofs, K. (2014a) Updating freeze: Aligning animal and human research. Neuroscience and Biobehavioral Reviews 47:165-76. [HM]

Hagenaars, M. A., Roelofs, K. \& Stins, J. F. (2014b) Human freezing in response to affective films. Anxiety, Stress and Coping 27(1):27-37. [HM]

Haggerty, D. C., Glykos, V., Adams, N. E. \& LeBeau, F. E. N. (2013) Bidirectional modulation of hippocampal gamma $(20-80 \mathrm{~Hz})$ frequency activity in vitro via alpha $(\alpha)$ - and beta $(\beta)$-adrenergic receptors (AR). Neuroscience 253(0):142-54 Available at: http://dx.doi.org/10.1016/j.neuroscience.2013.08.028. [aMM]

Hahn, S., Carlson, C., Singer, S. \& Gronlund, S. D. (2006) Aging and visual search: Automatic and controlled attentional bias to threat faces. Acta Psychologica 123:312-36. [KK]

Hahn, U. (2011) The problem of circularity in evidence, argument, and explanation. Perspectives on Psychological Science 6(2):172-82. [JRH]

Haider, B., Häusser, M. \& Carandini, M. (2013) Inhibition dominates sensory responses in the awake cortex. Nature 493(7430):97-100. [aMM]

Hajcak, G., MacNamara, A. \& Olvet, D. M. (2010) Event-related potentials, emotion, and emotion regulation: An integrative review. Developmental Neuropsychology 35(2):129-55. [DT]

Hamann, S. B., Ely, T. D., Grafton, S. T. \& Kilts, C. D. (1999) Amygdala activity related to enhanced memory for pleasant and aversive stimuli. Nature Neuroscience 2(3):289-93. [BR]

Han, J.-H., Kushner, S. A., Yiu, A. P., Cole, C. J., Matynia, A., Brown, R. A., Neve, R. L., Guzowski, J. F., Silva, A. J. \& Josselyn, S. A. (2007) Neuronal competition and selection during memory formation. Science 316(5823):457-60. [aMM]

Harley, C. W. (2007) Norepinephrine and the dentate gyrus. Progress in Brain Research 163:299-318. [CH]

Harley, C. W., Lalies, M. D. \& Nutt, D. J. (1996) Estimating the synaptic concentration of norepinephrine in dentate gyrus which produces $\beta$-receptor mediated long-lasting potentiation in vivo using microdialysis and intracerebroventricular norepinephrine. Brain Research 710(1):293-98. [aMM]

Harsay, H. A., Spaan, M., Wijnen, J. G. \& Ridderinkhof, K. R. (2012) Error awareness and salience processing in the oddball task: Shared neural mechanisms. Frontiers in Human Neuroscience 6:246. doi: 10.3389/ fnhum.2012.00246. [aMM]

Harsing, L. G. \& Matyus, P. (2013) Mechanisms of glycine release, which build up synaptic and extrasynaptic glycine levels: The role of synaptic and non-synaptic glycine transporters. Brain Research Bulletin 93:110-19. [aMM]

Hasselmo, M. E., Linster, C., Patil, M., Ma, D. \& Cekic, M. (1997) Noradrenergic suppression of synaptic transmission may influence cortical signal-to-noise ratio. Journal of Neurophysiology 77(6):3326-39. [aMM]

Hassert, D. L., Miyashita, T. \& Williams, C. L. (2004) The effects of peripheral vagal nerve stimulation at a memory-modulating intensity on norepinephrine output in the basolateral amygdala. Behavioral Neuroscience 118:79-88. [HDC]

Hatfield, T. \& McGaugh, J. L. (1999) Norepinephrine infused into the basolateral amygdala posttraining enhances retention in a spatial water maze task. Neurobiology of Learning and Memory 71(2):232-39. [aMM]

Havranek, M. M., Hulka, L. M., Tasiudi, E., Eisenegger, C., Vonmoos, M., Preller, K. H., Mossner, R., Baumgartner, M. R., Seifritz, E., Grunblattt, E. \& Quednow, B. B. (2015) $\alpha 2 \mathrm{~A}$-adrenergic receptor polymorphisms and mRNA expression levels are associated with delay discounting in cocaine users. Addiction Biology. doi: 10.1111/adb.12324. [RMT]

Hawrylycz, M. J., Lein, E. S., Guillozet-Bongaarts, A. L., Shen, E. H., Ng, L., Miller, J. A., van de Lagemaat, L. N., Smith, K. A., Ebbert, A., Riley, Z. L., Abajian, C., Beckmann, C. F., Bernard, A., Bertagnolli, D., Boe, A. F., Cartagena, P. M., Chakravarty, M. M., Chapin, M., Chong, J., Dalley, R. A., Daly, B. D., Dang, C., Datta, S., Dee, N., Dolbeare, T. A., Faber, V., Feng, D., Fowler, D. R., Goldy, J., Gregor, B. W., Haradon, Z., Haynor, D. R., Hohmann, J. G., Horvath, S., Howard, R. E., Jeromin, A., Jochim, J. M., Kinnunen, M., Lau, C., Lazarz, E. T., Lee, C., Lemon, T. A., Li, L., Li, Y., Morris, J. A., Overly, C. C., Parker, P. D., Parry, S. E., Reding, M., Royall, J. J., Schulkin, J., Sequeira, P. A., Slaughterbeck, C. R., Smith, S. C., Sodt, A. J., Sunkin, S. M., Swanson, B. E., Vawter, M. P., Williams, D., Wohnoutka, P., Zielke, H. R., Geschwind, D. H., Hof, P. R., Smith, S. M., Koch, C., Grant, S. G. \& Jones, A. R. (2012) An anatomically comprehensive atlas of the adult human brain transcriptome. Nature 489:391-99. [RMT]

Headley, D. B. \& Pare, D. (2013) In sync: Gamma oscillations and emotional memory. Frontiers in Behavioral Neuroscience 7. doi: 10.3389/ fnbeh.2013.00170. [aMM]

Headley, D. B. \& Weinberger, N. M. (2011) Gamma-band activation predicts both associative memory and cortical plasticity. The Journal of Neuroscience 31 (36):12748-58. doi: 10.1523/jneurosci.2528-11.2011. [aMM]

Headley, D. B. \& Weinberger, N. M. (2013) Fear conditioning enhances gamma oscillations and their entrainment of neurons representing the conditioned stimulus. The Journal of Neuroscience 33(13):5705-17. doi: 10.1523/jneurosci.4915-12.2013. [aMM]

Heerebout, B. T. \& Phaf, R. H. (2010a) Emergent oscillations in evolutionary simulations: Oscillating networks increase switching efficacy. Journal of Cognitive Neuroscience 22:807-23. [RHP]

Heerebout, B. T. \& Phaf, R. H. (2010b) Good vibrations switch attention: An affective function for network oscillations in evolutionary simulations. Cognitive, Affective and Behavioral Neuroscience 10:217-29. [RHP]

Heerebout, B. T., Tap, A. E. Y., Rotteveel, M. \& Phaf, R. H. (2013) Gamma flicker elicits positive affect without awareness. Consciousness and Cognition 22:28189. [RHP]

Helbig, T. D., Borod, J. C., Frisina, P. G., Tse, W., Voustianiouk, A., Olanow, C. W. \& Gracies, J.-M. (2011) Emotional processing affects movement speed. Journal of Neural Transmission 118(9):1319-22. [HM]

Hermans, E. J., Battaglia, F. P., Atsak, P., de Voogd, L. D., Fernández, G. \& Roozendaal, B. (2014) How the amygdala affects emotional memory by altering brain network properties. Neurobiology of Learning and Memory 112:2-16. [aMM, BR]

Hermans, E. J., van Marle, H. J. F., Ossewaarde, L., Henckens, M., Qin, S. Z., van Kesteren, M. T. R., Schoots, V. C., Cousijn, H., Rijpkema, M., Oostenveld, R. \& Fernandez, G. (2011) Stress-related noradrenergic activity prompts large-scale neural network reconfiguration. Science 334(6059):1151-53. doi: 10.1126/ science.1209603. [aMM, BR]

Herrero, I. \& Sánchez-Prieto, J. (1996) cAMP-dependent facilitation of glutamate release by $\beta$-adrenergic receptors in cerebrocortical nerve terminals. Journal of Biological Chemistry 271(48):30554-60. [aMM]

Hervé-Minvielle, A. \& Sara, S. J. (1995) Rapid habituation of auditory responses of locus coeruleus cells in anesthetized and awake rats. NeuroReport 6:45-50. [SJS]

Hillman, C. H., Rosengren, K. S. \& Smith, D. P. (2004) Emotion and motivated behavior: Postural adjustments to affective picture viewing. Biological Psychology 66(1):51-62. [HM]

Hirata, A., Aguilar, J. \& Castro-Alamancos, M. A. (2006) Noradrenergic activation amplifies bottom-up and top-down signal-to-noise ratios in sensory thalamus. 
References/Mather et al.: Norepinephrine ignites local hotspots of neuronal excitation

Journal of Neuroscience 26(16):4426-36. doi: 10.1523/jneurosci.529805.2006. [aMM]

Hommel, B. (1994) Spontaneous decay of response-code activation. Psychological Research 56:261-68. doi: 10.1007/BF00419656. [CMW]

Hopfield, J. J. (1982) Neural networks and physical systems with emergent collective computational abilities. Proceedings of the National Academy of Sciences of the United States of America 79:2554-58. [CH]

Horslen, B. C. \& Carpenter, M. G. (2011) Arousal, valence and their relative effects on postural control. Experimental Brain Research 215(1):27-34. [HM]

Howells, F. M., Stein, D. J. \& Russell, V. A. (2010) Perceived mental effort correlates with changes in tonic arousal during attentional tasks. Behavioral and Brain Functions 6:39. doi: 10.1186/1744-9081-6-39. [RG]

Hu, H., Real, E., Takamiya, K., Kang, M.-G., Ledoux, J., Huganir, R. L. \& Malinow, R. (2007) Emotion enhances learning via norepinephrine regulation of AMPAreceptor trafficking. Cell 131(1):160-73. [BAS]

Hu, P., Stylos-Allan, M. \& Walker, M. P. (2006) Sleep facilitates consolidation of emotional declarative memory. Psychological Science 17(10):891-98. [aMM]

Huang, S., Huganir, R. L. \& Kirkwood, A. (2013) Adrenergic gating of Hebbian spike-timing-dependent plasticity in cortical interneurons. The Journal of Neuroscience 33(32):13171-78. doi: 10.1523/jneurosci.5741-12.2013. [aMM]

Huang, S., Rozas, C., Treviño, M., Contreras, J., Yang, S., Song, L., Yoshioka, T., Lee, H.-K. \& Kirkwood, A. (2014) Associative Hebbian synaptic plasticity in primate visual cortex. The Journal of Neuroscience 34(22):7575-79. [aMM]

Huang, Y. \& Rao, R. P. N. (2011) Predictive coding. Wiley Interdisciplinary Reviews: Cognitive Science 2:580-93. doi: 10.1002/wcs.142. [FF-S]

Hurlemann, R. (2006) Noradrenergic control of emotion-induced amnesia and hypermnesia. Reviews in the Neurosciences 17(5):525-32. Available at: http:// www.ncbi.nlm.nih.gov/pubmed/17180877. [RH]

Hurlemann, R. (2008) Noradrenergic-glucocorticoid mechanisms in emotioninduced amnesia: From adaptation to disease. Psychopharmacology 197(1):1323. Available at: http://www.ncbi.nlm.nih.gov/pubmed/18038126. [RH, rMM]

Hurlemann, R., Hawellek, B., Matusch, A., Kolsch, H., Wollersen, H., Madea, B. Vogeley, K., Maier, W. \& Dolan, R. J. (2005) Noradrenergic modulation of emotion-induced forgetting and remembering. Journal of Neuroscience 25 (27):6343-49. Available at: http://www.ncbi.nlm.nih.gov/pubmed/16000624. $[\mathrm{RH}]$

Hurlemann, R., Matusch, A., Hawellek, B., Klingmuller, D., Kolsch, H., Maier, W. \& Dolan, R. J. (2007a) Emotion-induced retrograde amnesia varies as a function of noradrenergic-glucocorticoid activity. Psychopharmacology 194(2):261-69. Available at: http://www.ncbi.nlm.nih.gov/pubmed/17588225. [RH]

Hurlemann, R., Rehme, A. K., Diessel, M., Kukolja, J., Maier, W., Walter, H. \& Cohen, M. X. (2008) Segregating intra-amygdalar responses to dynamic facial emotion with cytoarchitectonic maximum probability maps. 1 Journal of Neuroscience Methods 172(1):13-20. Available at: http://www.ncbi.nlm.nih.gov/ pubmed/18486975. [RH]

Hurlemann, R., Wagner, M., Hawellek, B., Reich, H., Pieperhoff, P., Amunts, K., Oros-Peusquens, A. M., Shah, N. J., Maier, W. \& Dolan, R. J. (2007b) Amygdala control of emotion-induced forgetting and remembering. Neuropsychologia 45 (5):877-84. Available at: http://www.ncbi.nlm.nih.gov/pubmed/17027866. $[\mathrm{RH}]$

Hurlemann, R., Walter, H., Rehme, A. K., Kukolja, J., Santoro, S. C., Schmidt, C., Schnell, K., Musshoff, F., Keysers, C., Maier, W., Kendrick, K. M. \& Onur, O. A. (2010) Human amygdala reactivity is diminished by the beta-noradrenergic antagonist propranolol. Psychological Medicine 40(11):1839-48. Available at: http://www.ncbi.nlm.nih.gov/pubmed/20102667. [RH]

Hurley, L., Devilbiss, D. \& Waterhouse, B. (2004) A matter of focus: Monoaminergic modulation of stimulus coding in mammalian sensory networks. Current Opinion in Neurobiology 14(4):488-95. [aMM]

Hutchinson, J. B. \& Turk-Browne, N. B. (2012) Memory-guided attention: Control from multiple memory systems. Trends in Cognitive Sciences 16(12):576-79. [aMM]

Ishigaki, H., Miyao, M. \& Ishihara, S. Y. (1991) Change of pupil size as a function of exercise. Journal of Human Ergology 20(1):61-66. [rMM]

Ito, T. A., Cacioppo, J. T. \& Lang, P. J. (1998) Eliciting affect using the International Affective Picture System: Trajectories through evaluative space. Personality and Social Psychology Bulletin 24:855-79. [KK]

Itti, L. \& Koch, C. (2000) A saliency-based search mechanism for overt and covert shifts of visual attention. Vision Research 40(10-12):1489-506. doi: 10.1016/ S0042-6989(99)00163-7. [aMM]

Jacobs, B. L. (1986) Single unit activity of locus coeruleus neurons in behaving animals. Progress in Neurobiology 27(2):183-94. [SB]

James, W. (1913) The principles of psychology, vol 2. Henry Holt. [SB]

Jasper, J. R., Lesnick, J. D., Chang, L. K., Yamanishi, S. S., Chang, T. K., Hsu, S. A., Daunt, D. A., Bonhaus, D. W. \& Eglen, R. M. (1998) Ligand efficacy and potency at recombinant alpha2 adrenergic receptors: Agonist-mediated [35S] GTPgammaS binding. Biochemistry and Pharmacology 55:1035-43. [RMT]
Javoy-Agid, F., Scatton, B., Ruberg, M., L'heureux, R., Cervera, P., Raisman, R., Maloteaux, J.-M., Beck, H. \& Agid, Y. (1989) Distribution of monoaminergic, cholinergic, and GABAergic markers in the human cerebral cortex. Neuroscience 29(2):251-59. [aMM]

Jepma, M. \& Nieuwenhuis, S. (2011) Pupil diameter predicts changes in the exploration-exploitation trade-off: Evidence for the adaptive gain theory. Journal of Cognitive Neuroscience 23:1587-96. Available at: http://doi.org/10. 1162/jocn.2010.21548. [SB, MRN]

Ji, X.-H., Cao, X.-H., Zhang, C.-L., Feng, Z.-J., Zhang, X.-H., Ma, L. \& Li, B.-M. (2008) Pre- and postsynaptic $\beta$-adrenergic activation enhances excitatory synaptic transmission in layer V/VI pyramidal neurons of the medial prefrontal cortex of rats. Cerebral Cortex 18(7):1506-20. doi: 10.1093/cercor/bhm177. [aMM]

Jodo, E., Chiang, C. \& Aston-Jones, G. (1998) Potent excitatory influence of prefrontal cortex activity on noradrenergic locus coeruleus neurons. Neuroscience 83(1):63-79. Available at: http://dx.doi.org/10.1016/S0306-4522(97)00372-2. [aMM]

Joëls, M., Fernandez, G. \& Roozendaal, B. (2011) Stress and emotional memory: A matter of timing. Trends in Cognitive Sciences 15(6):280-88. [rMM]

Johansen, J. P., Diaz-Mataix, L., Hamanaka, H., Ozawa, T., Ycu, E., Koivumaa, J., Kumar, A., Hou, M., Deisseroth, K. \& Boyden, E. S. (2014) Hebbian and neuromodulatory mechanisms interact to trigger associative memory formation. Proceedings of the National Academy of Sciences of the United States of America 111(51):E5584-92. [aMM]

Jones, B. E. (2004) Activity, modulation and role of basal forebrain cholinergic neurons innervating the cerebral cortex. Progress in Brain Research 145:15769. [aMM]

Jones, B. E. (2011) Neurobiology of waking and sleeping. Handbook of Clinical Neurology 98:131-49. doi: 10.1016/B978-0-444-52006-7.00009-5. [AB]

Jones, B. E. \& Hassani, O. K. (2008) The role of Hcrt/Orx and MCH neurons in sleep-wake state regulation. Sleep 36:1769-72. doi: 10.5665/sleep.3188. [AB]

Jones, B. E. \& Moore, R. Y. (1977) Ascending projections of the locus coeruleus in the rat: II. Autoradiographic study. Brain Research 127(1):23-53. Available at: http://dx.doi.org/10.1016/0006-8993(77)90378-X. [aMM]

Jones, S. M., Snell, L. D. \& Johnson, K. M. (1987) Phencyclidine selectively inhibits $\mathrm{N}$-methyl-D-aspartate-induced hippocampal $[3 \mathrm{H}]$ norepinephrine release. Journal of Pharmacology and Experimental Therapeutics 240(2):492-97. [aMM]

Joshi, S., Li, Y., Kalwani, R. \& Gold, J. I. (2016) Relationships between pupil diameter and neuronal activity in the locus coeruleus, colliculi, and cingulate cortex. Neuron 89:221-34. [MRN]

Kalaria, R., Andorn, A., Tabaton, M., Whitehouse, P., Harik, S. \& Unnerstall, J. (1989) Adrenergic receptors in aging and Alzheimer's Disease: Increased $\beta_{2^{-}}$ receptors in prefrontal cortex and hippocampus. Journal of Neurochemistry 53 (6):1772-81. [aMM]

Kalpachidou, T., Raftogianni, A., Melissa, P., Kollia, A.-M., Stylianopoulou, F. \& Stamatakis, A. (2015) Effects of a neonatal experience involving reward through maternal contact on the noradrenergic system of the rat prefrontal cortex. Cerebral Cortex 26(9):3866-77. doi: 10.1093/cercor/bhv192. [RG]

Kandel, E. R. (2012) The molecular biology of memory: cAMP, PKA, CRE, CREB1, CREB-2, and CPEB. Molecular Brain 5(1):14. [aMM]

Kaplan, R. L., Van Damme, I. \& Levine, L. J. (2012) Motivation matters: Differing effects of pre-goal and post-goal emotions on attention and memory. Frontiers in Psychology 3: 404. [aMM]

Karst, H., Berger, S., Turiault, M., Tronche, F., Schütz, G. \& Joëls, M. (2005) Proceedings of the National Academy of Sciences of the United States of America 102(52):19204-207. Available at: http://www.ncbi.nlm.nih.gov/pubmed/ 16361444. [RH]

Kaspar, K. (2013) What guides overt attention under natural conditions? Past and future research. ISRN Neuroscience Article ID 868491:1-8. [KK]

Kaspar, K. \& König, P. (2012) Emotions and personality traits as high-level factors in visual attention: A review. Frontiers in Human Neuroscience 6:321. [KK]

Kaspar, K., Ramos Gameiro, R. \& König, P. (2015) Feeling good, searching the bad: Positive priming increases attention and memory for negative stimuli on webpages. Computers in Human Behavior 53:332-43. [KK]

Katsuki, H., Izumi, Y. \& Zorumski, C. F. (1997) Noradrenergic regulation of synaptic plasticity in the hippocampal CA1 region. Journal of Neurophysiology 77 (6):3013-20. [aMM]

Keil, A. \& Ihssen, N. (2004) Identification facilitation for emotionally arousing verbs during the attentional blink. Emotion 4(1):23-35. doi: 10.1037/15283542.4.1.23. [aMM]

Keitel, C., Andersen, S. K., Quigley, C. \& Müller, M. M. (2013) Independent effects of attentional gain control and competitive interactions on visual stimulus processing. Cerebral Cortex 23(4):940-46. [aMM]

Kemp, A. \& Manahan-Vaughan, D. (2008) $\beta$-adrenoreceptors comprise a critical element in learning-facilitated long-term plasticity. Cerebral Cortex 18 (6):1326-34. [BAS] 
Kendrick, K. M., Levy, F. \& Keverne, E. B. (1992) Changes in the sensory processing of olfactory signals induced by birth in sheep. Science 256(5058):83336. $[\mathrm{RG}]$

Kennedy, B. L. \& Most, S. B. (2012) Perceptual, not memorial, disruption underlies emotion-induced blindness. Emotion 12(2):199-202. [aMM]

Kensinger, E. A. (2004) Remembering emotional experiences: The contribution of valence and arousal. Reviews in the Neurosciences 15(4):241-51. [aMM]

Kensinger, E. A. (2008) Age differences in memory for arousing and nonarousing emotional words. Journal of Gerontology: Psychological Sciences 63:P13P18. [rMM]

Kensinger, E. A., Garoff-Eaton, R. J. \& Schacter, D. L. (2007) Effects of emotion on memory specificity: Memory trade-offs elicited by negative visually arousing stimuli. Journal of Memory and Language 56(4):575-91. doi: 10.1016/j. jml.2006.05.004. [aMM]

Kilpatrick, L. \& Cahill, L. (2003) Amygdala modulation of parahippocampal and frontal regions during emotionally influenced memory storage. NeuroImage 20 (4):2091-99. [aMM]

Kim, J. J. \& Yoon, K. S. (1998) Stress: Metaplastic effects in the hippocampus. Trends in Neurosciences 21(12):505-509. [BAS]

Kim, M. A., Lee, H. S., Lee, B. Y. \& Waterhouse, B. D. (2004) Reciprocal connections between subdivisions of the dorsal raphe and the nuclear core of the locus coeruleus in the rat. Brain Research 1026(1):56-67. [QG]

Kim, S. \& Hamann, S. B. (2007) Neural correlates of positive and negative emotion regulation. Journal of Cognitive Neuroscience 19:776-98. [KK]

Kimura, F. \& Nakamura, S. (1985) Locus coeruleus neurons in the neonatal rat: Electrical activity and responses to sensory stimulation. Developmental Brain Research 23(2):301-305. Available at: http://dx.doi.org/10.1016/0165-3806(85) 90055-0. [RG]

Kitchigina, V., Vankov, A., Harley, C. \& Sara, S. J. (1997) Novelty-elicited, noradrenaline-dependent enhancement of excitability in the dentate gyrus. European Journal of Neuroscience 9:41-47. [CH]

Klein, R. M. \& Ivanoff, J. (2011) The components of visual attention and the ubiquitous Simon effect. Acta Psychologica 136:225-34. doi: 10.1016/j actpsy.2010.08.003. [CMW]

Klimek, V., Stockmeier, C., Overholser, J., Meltzer, H. Y., Kalka, S., Dilley, G. \& Ordway, G. A. (1997) Reduced levels of norepinephrine transporters in the locus coeruleus in major depression. Journal of Neuroscience 17:8451-58. [CGA]

Klink, P. C., Jentgens, P. \& Lorteije, J. A. (2014) Priority maps explain the roles of value, attention, and salience in goal-oriented behavior. The Journal of Neuroscience 34(42):13867-69. [aMM]

Klumpers, F., Morgan, B., Terburg, D., Stein, D. J. \& van Honk, J. (2014) Impaired acquisition of classically conditioned fear-potentiated startle reflexes in humans with focal bilateral basolateral amygdala damage. Social Cognitive and Affective Neuroscience 10(9):1161-68. [BR, rMM]

Knight, M. \& Mather, M. (2009) Reconciling findings of emotion-induced memory enhancement and impairment of preceding items. Emotion 9(6):763-81. doi: 10.1037/a0017281. [arMM, BAS]

Knight, M., Seymour, T. L., Gaunt, J. T., Baker, C., Nesmith, K. \& Mather, M (2007) Aging and goal-directed emotional attention: Distraction reverses emotional biases. Emotion 7(4):705-14. doi: 10.1037/1528-3542.7.4.705. [arMM, KK]

Kobayashi, M., Kojima, M., Koyanagi, Y., Adachi, K., Imamura, K. \& Koshikawa, N. (2009) Presynaptic and postsynaptic modulation of glutamatergic synaptic transmission by activation of $\alpha \mathrm{l}$ - and $\beta$-adrenoceptors in layer $\mathrm{V}$ pyramidal neurons of rat cerebral cortex. Synapse 63(4):269-81. doi: 10.1002/ syn.20604. [aMM]

Koch, C. \& Ullman, S. (1984) Selecting one among the many: A simple network implementing shifts in selective visual attention. MIT Artificial Intelligence Laboratory Technical Report Memo $770 . \quad[\mathrm{CH}]$

Koch, C. \& Ullman, S. (1987) Shifts in selective visual attention: Towards the underlying neural circuitry. In: Matters of intelligence, ed. L. M. Vaina, pp. 11541. Springer. $[\mathrm{CH}]$

Kocsis, B., Li, S. \& Hajos, M. (2007) Behavior-dependent modulation of hippocampal EEG activity by the selective norepinephrine reuptake inhibitor reboxetine in rats. Hippocampus 17(8):627-33. doi: 10.1002/hipo.20299. $[\mathrm{aMM}]$

Kolta, A., Diop, L. \& Reader, TA (1987) Noradrenergic effects on rat visual cortex: Single-cell microiontophoretic studies of alpha-2 adrenergic receptors. Life Sciences 20:281-89. [QG]

Korotkova, T., Fuchs, E. C., Ponomarenko, A., von Engelhardt, J. \& Monyer, H. (2010) NMDA receptor ablation on parvalbumin-positive interneurons impairs hippocampal synchrony, spatial representations, and working memory. Neuron 68(3):557-69. Available at: http://dx.doi.org/10.1016/j.neuron.2010.09.017. [aMM]

Koss, M. C., Gherezghiher, T. \& Nomura, A. (1984) CNS adrenergic inhibition of parasympathetic oculomotor tone. Journal of the Autonomic Nervous System 10 (1):55-68. [rMM
Krauseneck, T., Padberg, F., Roozendaal, B., Grathwohl, M., Weis, F., Hauer, D., Kaufmann, I., Schmoeckel, M. \& Schelling, G. (2010) A $\beta$-adrenergic antagonist reduces traumatic memories and PTSD symptoms in female but not in male patients after cardiac surgery. Psychological Medicine 40(05):861-69. [rMM]

Krebs, R. M., Fias, W., Achten, E. \& Boehler, C. N. (2013) Picture novelty attenuates semantic interference and modulates concomitant neural activity in the anterior cingulate cortex and the locus coeruleus. NeuroImage 74(0):179-87. Available at: http://dx.doi.org/10.1016/j.neuroimage.2013.02.027. [aMM]

Krugers, H. J., Karst, H. \& Joels, M. (2012) Interactions between noradrenaline and corticosteroids in the brain: From electrical activity to cognitive performance. Frontiers in Cellular Neuroscience 6:Article 15. [rMM]

Krystal, J. H., Sanacora, G. \& Duman, R. S. (2013) Rapid-acting glutamatergic antidepressants: The path to ketamine and beyond. Biological Psychiatry 73:1133-41. [CGA]

Kuhbandner, C. \& Zehetleitner, M. (2011) Dissociable effects of valence and arousal in adaptive executive control. PLoS ONE 6(12):e29287. [arMM]

Kuhl, P. K. (2007) Is speech learning "gated" by the social brain? Developmental Science 10(1):110-20. doi: 10.1111/j.1467-7687.2007.00572.x. [RG]

Kuhl, P. K., Williams, K. A., Lacerda, F., Stevens, K. N. \& Lindblom, B. (1992) Linguistic experience alters phonetic perception in infants by 6 months of age. Science 255(5044):606-608. doi: 10.1126/science.1736364. [RG]

Kuhlmann, S. \& Wolf, O. T. (2006) Arousal and cortisol interact in modulating memory consolidation in healthy young men. Behavioral Neuroscience 120(1):217-23. [BR]

Kukolja, J., Klingmuller, D., Maier, W., Fink, G. R. \& Hurlemann, R. (2011) Noradrenergic-glucocorticoid modulation of emotional memory encoding in the human hippocampus. Psychological Medicine 41(10):2167-76. Available at: http://www.ncbi.nlm.nih.gov/pubmed/21375794. [RH, rMM]

Kukolja, J., Schlapfer, T. E., Keysers, C., Klingmuller, D., Maier, W., Fink, G. R. \& Hurlemann, R. (2008) Modeling a negative response bias in the human amygdala by noradrenergic-glucocorticoid interactions. Journal of Neuroscience 28 (48):12868-76. Available at: http://www.ncbi.nlm.nih.gov/pubmed/ 19036981. [RH, rMM]

Kuo, S. P. \& Trussell, L. O. (2011) Spontaneous spiking and synaptic depression underlie noradrenergic control of feed-forward inhibition. Neuron 71(2):30618. $[\mathrm{aMM}]$

LaBar, K. S. \& Cabeza, R. (2006) Cognitive neuroscience of emotional memory. Nature Reviews Neuroscience 7(1):54-64. [aMM]

Lakatos, P., Karmos, G., Mehta, A. D., Ulbert, I. \& Schroeder, C. E. (2008) Entrainment of neuronal oscillations as a mechanism of attentional selection. Science 320(5872):110-13. [aMM]

Lalies, M., Middlemiss, D. N. \& Ransom, R. (1988) Stereoselective antagonism of NMDA-stimulated noradrenaline release from rat hippocampal slices by MK801. Neuroscience Letters 91(3):339-42. [arMM]

Lally, N., Mullins, P. G., Roberts, M. V., Price, D., Gruber, T. \& Haenschel, C. (2014) Glutamatergic correlates of gamma-band oscillatory activity during cognition: A concurrent ER-MRS and EEG study. NeuroImage 85:823-33. [aMM]

LaLumiere, R. T., Buen, T. V. \& McGaugh, J. L. (2003) Post-training intra-basolateral amygdala infusions of norepinephrine enhance consolidation of memory for contextual fear conditioning. Journal of Neuroscience 23(17):6754-58. [aMM]

Lambertz, M. \& Langhorst, P. (1995) Cardiac rhythmic patterns in neuronal activity are related to the firing rate of the neurons: I. Brainstem reticular neurons of dogs. Journal of the Autonomic Nervous System 51:153-63. [HDC]

Lambertz, M., Schulz, G. \& Langhorst, P. (1995) Cardiac rhythmic patterns in neuronal activity related to the firing rate of the neurons: II. Amygdala neurons of cats. Journal of the Autonomic Nervous System 51:165-73. [HDC]
[HD

Lang, P. J., Greenwald, M. K., Bradley, M. M. \& Hamm, A. O. (1993) Looking at pictures: Affective, facial, visceral, and behavioral reactions. Psychophysiology 30:261-73. [AM]

Langer, S. Z. (2008) Presynaptic autoreceptors regulating transmitter release. Neurochemistry International 52(1/2):26-30. Available at: http://dx.doi.org/10.1016/ j.neuint.2007.04.031. [aMM]

Larkum, M. (2013) A cellular mechanism for cortical associations: An organizing principle for the cerebral cortex. Trends in Neuroscience 36:141-51. [MEL]

Larkum, M. E. Nevian, T., Sandler, M., Polsky, A. \& Schiller, J. (2009) Synaptic integration in tuft dendrites of layer 5 pyramidal neurons: A new unifying principle. Science 325:756-60. [MEL]

Larkum, M. E., Waters, J., Sakmann, B. \& Helmchen, F. (2007) Dendritic spikes in apical dendrites of neocortical layer $2 / 3$ pyramidal neurons. The Journal of Neuroscience 27:8999-9008. [MEL]

Larkum, M. E., Zhu, J. J. \& Sakmann, B. (1999) A new cellular mechanism for coupling inputs arriving at different cortical layers. Nature 98 (6725):338-41. [MEL]

Lavín, C., San Martín, R. \& Rosales Jubal, E. (2014) Pupil dilation signals uncertainty and surprise in a learning gambling task. Frontiers in Behavioral Neuroscience 7:218. [MRN] 
References/Mather et al.: Norepinephrine ignites local hotspots of neuronal excitation

Lavzin, M., Rapoport, S., Polsky, A., Garion, L. \& Schiller, J. (2012) Nonlinear dendritic processing determines angular tuning of barrel cortex neurons in vivo. Nature 490:397-401. [MEL]

Lecas, J. C. (2001) Noradrenergic modulation of tactile responses in rat cortex: Current source-density and unit analyses. Comptes Rendus de l'Académie des Sciences - III 324:33-44. [QG]

Lecas, J. C. (2004) Locus coeruleus activation shortens synaptic drive while decreasing spike latency and jitter in sensorimotor cortex: Implications for neuronal integration. European Journal of Neuroscience 19:2519-30. [QG]

Leclerc, C. M. \& Kensinger, E. A. (2008) Effects of age on detection of emotional information. Psychology and Aging 23(1):209-15. [arMM]

Lee, J. L., Milton, A. L. \& Everitt, B. J. (2006) Reconsolidation and extinction of conditioned fear: Inhibition and potentiation. The Journal of Neuroscience 26 (39):10051-56. [aMM]

Lee, M. G., Hassani, O. K., Alonso, A. \& Jones, B. E. (2005) Cholinergic basal forebrain neurons burst with theta during waking and paradoxical sleep. Journal of Neuroscience 25:4365-69. doi: 10.1523/JNEUROSCI.0178-05.2005. [AB]

Lee, T. H., Baek, J., Lu, Z. L. \& Mather, M. (2014a) How arousal modulates the contrast sensitivity function. Emotion 5:978-84. [aMM]

Lee, T. H., Greening, S. G. \& Mather, M. (2015) Encoding on goal-relevant stimuli is strengthened by emotional stimuli in memory. Frontiers in Psychology 6:1173. [rMM]

Lee, T. H., Itti, L. \& Mather, M. (2012) Evidence for arousal-biased competition in perceptual learning. Frontiers in Emotion Science 3:241. [aMM]

Lee, T. H., Sakaki, M., Cheng, R., Velasco, R. \& Mather, M. (2014b) Emotional arousal amplifies the effects of biased competition in the brain. Social Cognitive and Affective Neuroscience 9(12):2067-77. doi: 10.1093/scan/nsu015. [arMM]

Lehmann, J., Valentino, R. \& Robine, V. (1992) Cortical norepinephrine release elicited in situ by $N$-methyl-D-aspartate (NMDA) receptor stimulation: A microdialysis study. Brain Research 599(1):171-74. Available at: http://dx.doi. org/10.1016/0006-8993(92)90868-A. [aMM]

Lester, R. A., Clements, J. D., Westbrook, G. L. \& Jahr, C. E. (1990) Channel kinetics determine the time course of NMDA receptor-mediated synaptic currents. Nature 346:565-67. [rMM]

Leutgeb, J. K., Leutgeb, S., Moser, M.-B. \& Moser, E. I. (2007) Pattern separation in the dentate gyrus and CA3 of the hippocampus. Science 315:961-66. [CH]

Levine, L. J. \& Edelstein, R. S. (2009) Emotion and memory narrowing: A review and goal-relevance approach. Cognition and Emotion 23(5):833-75. doi: 10.1080/02699930902738863. [aMM]

Levitt, P., Rakic, P. \& Goldman-Rakic, P. (1984) Region-specific distribution of catecholamine afferents in primate cerebral cortex: A fluorescence histochemical analysis. The Journal of Comparative Neurology 227(1):23-36. doi: 10.1002/ cne.902270105. [aMM]

Levy, W. B. \& Steward, O. (1979) Synapses as associative memory elements in the hippocampal formation. Brain Research 175:233-45. [CH]

Li, B.-M. \& Mei, Z.-T. (1994) Delayed response deficit induced by local injection of the alpha-2 adrenergic antagonist yohimbine into the dorsolateral prefrontal cortex in young adult monkeys. Behavioral and Neural Biology 62:134-39. [CGA]

Liddell, B. J., Brown, K. J., Kemp, A. H., Barton, M. J., Das, P., Peduto, A., Gordon, E. \& Williams, L. M. (2005) A direct brainstem-amygdala-cortical "alarm" system for subliminal signals of fear. NeuroImage 24(1):235-43. [aMM]

Lim, S. L., Padmala, S. \& Pessoa, L. (2009) Segregating the significant from the mundane on a moment-to-moment basis via direct and indirect amygdala contributions. Proceedings of the National Academy of Sciences of the United States of America 106(39):16841-46. doi: 10.1073/pnas.0904551106. [aMM]

Lindgren, M. E., Fagundes, C. P., Alfano, C. M., Povoski, S. P., Agnese, D. M., Arnold, M. W., Farrar, W. B., Yee, L. D., Carson, W. E. \& Schmidt, C. R. (2013) Beta blockers may reduce intrusive thoughts in newly diagnosed cancer patients. Psychooncology 22(8):1889-94. [rMM]

Lindquist, K. A., Wager, T. D., Kober, H., Bliss-Moreau, E. \& Barrett, L. F. (2012) The brain basis of emotion: A meta-analytic review. Behavioral and Brain Sciences 35:121-202. [TDH]

Lisman, J., Grace, A. A. \& Duzel, E. (2011) A neoHebbian framework for episodic memory; role of dopamine-dependent late LTP. Trends in Neurosciences 34 (10):536-47. [MR]

Liu, D. L. J., Graham, S. \& Zorawski, M. (2008) Enhanced selective memory consolidation following post-learning pleasant and aversive arousal. Neurobiology of Learning and Memory 89(1):36-46. doi: 10.1016/j.nlm.2007.09.001. [aMM]

Liu, T.-L., Chen, D.-Y. \& Liang, K. (2009) Post-training infusion of glutamate into the bed nucleus of the stria terminalis enhanced inhibitory avoidance memory: An effect involving norepinephrine. Neurobiology of Learning and Memory 91 (4):456-65. [aMM]

Livingstone, M. S. \& Hubel, D. H. (1981) Effects of sleep and arousal on the processing of visual information in the cat. Nature 291(5816):554-61. [aMM]

Loftus, E. F. (1975) Leading questions and the eyewitness report. Cognitive Psychology 7(4):560-72. [JRH]
Long, N. M., Danoff, M. S. \& Kahana, M. J. (2015) Recall dynamics reveal the retrieval of emotional context. Psychonomic Bulleting and Review 22(5):1328-33. [DT]

Lovitz, E. S. \& Thompson, L. T. (2015) Memory-enhancing intra-basolateral amygdala clenbuterol infusion reduces post-burst afterhyperpolarizations in hippocampal CA1 pyramidal neurons following inhibitory avoidance learning. Neurobiology of Learning and Memory 119(1):34-41. [BR, rMM]

Luccini, E., Musante, V., Neri, E., Brambilla Bas, M., Severi, P., Raiteri, M. \& Pittaluga, A. (2007) Functional interactions between presynaptic NMDA receptors and metabotropic glutamate receptors co-expressed on rat and human noradrenergic terminals. British Journal of Pharmacology 151(7):1087-94. [aMM]

Luczak, A., Bartho, P. \& Harris, K. D. (2013) Gating of sensory input by spontaneous cortical activity. The Journal of Neuroscience 33(4):1684-95. [aMM]

Lüscher, C. \& Malenka, R. C. (2012) NMDA receptor-dependent long-term potentiation and long-term depression (LTP/LTD). Cold Spring Harbor Perspectives in Biology 4(6). doi: 10.1101/cshperspect.a005710. [aMM]

Ly, V., Huys, Q. J., Stins, J. F., Roelofs, K. \& Cools, R. (2014) Individual differences in bodily freezing predict emotional biases in decision making. Frontiers in Behavioral Neuroscience 8:Article 237. [HM]

Lynch, M. (2004) Long-term potentiation and memory. Physiological Reviews 84 (1):87-136. [aMM]

Lynch, M. \& Bliss, T. (1986) Noradrenaline modulates the release of $\left[{ }^{14} \mathrm{C}\right]$ glutamate from dentate but not from CA1/CA3 slices of rat hippocampus. Neuropharmacology 25:493-98. [CH]

MacKay, D. G., Shafto, M., Taylor, J. K., Marian, D. E., Abrams, L. \& Dyer, J. R. (2004) Relations between emotion, memory, and attention: Evidence from taboo Stroop, lexical decision, and immediate memory tasks. Memory and Cognition 32(3):474-88. [aMM]

MacLeod, J. W., Lawrence, M. A., McConnell, M. M., Eskes, G. A., Klein, R. M. \& Shore, D. I. (2010) Appraising the ANT: Psychometric and theoretical considerations of the Attention Network Test. Neuropsychology 24(5):637. [CMW]

Madison, D. \& Nicoll, R. (1982) Noradrenaline blocks accommodation of pyramidal cell discharge in the hippocampus. Nature 299:636-38. [aMM]

Magistretti, P. J., Morrison, J. H., Shoemaker, W. J., Sapin, V. \& Bloom, F. E. (1981) Vasoactive intestinal polypeptide induces glycogenolysis in mouse cortical slices: A possible regulatory mechanism for the local control of energy metabolism. Proceedings of the National Academy of Sciencesof the United States of America 78(10):6535-39. [aMM]

Maity, S., Jarome, T. J., Blair, J., Lubin, F. D. \& Nguyen, P. V. (2015) Norepinephrine goes nuclear: Epigenetic modifications during long-lasting synaptic potentiation triggered by activation of beta-adrenergic receptors. The Journal of Physiology 594(4):863-81. [rMM]

Manaye, K. F., McIntire, D. D., Mann, D. M. A. \& German, D. C. (1995) Locuscoeruleus cell loss in the aging human brain: A nonrandom process. Journal of Comparative Neurology 358(1):79-87. doi: 10.1002/cne.903580105. [aMM, MRN]

Manunta, Y. \& Edeline, J.-M. (1997) Effects of noradrenaline on frequency tuning of auditory cortex neurons. European Journal of Neuroscience 9:833-47. [QG]

Manunta, Y. \& Edeline, J.-M. (1998) Effects of noradrenaline on rate-level function of auditory cortex neurons: Is there a gating effect of noradrenaline? Experimental Brain Research 118:361-72. [QG]

Manunta, Y. \& Edeline, J.-M. (1999) Effects of norepinephrine on frequency tuning of auditory cortex neurons during wakefulness and slow wave sleep. European Journal of Neuroscience 11:2134-50. [QG]

Manunta, Y. \& Edeline, J. M. (2004) Noradrenergic induction of selective plasticity in the frequency tuning of auditory cortex neurons. Journal of Neurophysiology 92:1445-63. [QG]

Marchi, M. \& Grilli, M. (2010) Presynaptic nicotinic receptors modulating neurotransmitter release in the central nervous system: Functional interactions with other coexisting receptors. Progress in Neurobiology 92:105-11. doi: 10.1016/j pneurobio.2010.06.004. [AB]

Marien, M. R., Colpaert, F. C. \& Rosenquist, A. C. (2004) Noradrenergic mechanisms in neurodegenerative diseases: a theory. Brain Research Reviews 45(1):38-78. [rMM]

Markovic, J., Anderson, A. K. \& Todd, R. M. (2014) Tuning to the significant: Neura and genetic processes underlying affective enhancement of visual perception and memory. Behavioural Brain Research 259:229-41. [aMM, RMT]

Marr, D. (1971) Simple memory: A theory for archicortex. Philosophical Transactions of the Royal Society B: Biological Sciences 262:23-81. [CH]

Marrocco, R. T., Lane, R. F., McClurkin, J. W., Blaha, C. D. \& Alkire, M. F. (1987) Release of cortical catecholamines by visual stimulation requires activity in thalamocortical afferents of monkey and cat. The Journal of Neuroscience 7:2756-67. [EE, RN]

Martins, A. R. \& Froemke, R. C. (2015) Coordinated forms of noradrenergic plasticity in the locus coeruleus and primary auditory cortex. Nature Neuroscience 18(10):1483-92. [QG]

Marzo, A., Bai, J. \& Otani, S. (2009) Neuroplasticity regulation by noradrenaline in mammalian brain. Current Neuropharmacology 7(4):286. [aMM] 
Marzo, A., Totah, N. K., Neves, R. M., Logothetis, N. K. \& Eschenko, O. (2014) Unilateral electrical stimulation of rat locus coeruleus elicits bilateral response of norepinephrine neurons and sustained activation of medial prefrontal cortex. Journal of Neurophysiology 111(12):2570-88. doi: 10.1152/jn.00920.2013. [aMM]

Mather, M. (2007) Emotional arousal and memory binding: An object-based framework. Perspectives on Psychological Science 2(1):33-52. doi: 10.1111/ j.1745-6916.2007.00028.x. [aMM]

Mather, M. \& Knight, M. (2008) The emotional harbinger effect: Poor context memory for cues that previously predicted something arousing. Emotion 8 (6):850-60. [rMM]

Mather, M. \& Harley, C. W. (2016) The locus coeruleus: Essential for maintaining cognitive function and the aging brain. Trends in Cognitive Sciences 20:21426. [aMM]

Mather, M. \& Knight, M. R. (2006) Angry faces get noticed quickly: Threat detection is not impaired among older adults. Journals of Gerontology Series B: Psychological Sciences and Social Sciences 61:P54-P57. [arMM]

Mather, M. \& Sutherland, M. R. (2011) Arousal-biased competition in perception and memory. Perspectives on Psychological Science 6(2):114-33. doi: 10.1177/ 1745691611400234. [aMM, AM, BR]

McCormick, D. A. \& Prince, D. A. (1986) Mechanisms of action of acetylcholine in the guinea-pig cerebral cortex in vitro. Journal of Physiology 375:169-94. doi: 10.1113/jphysiol.1986.sp016112. [AB]

McEwen, B. S., Bowles, N. P., Gray, J. D., Hill, M. N., Hunter, R. G., Karatsoreos, I. N. \& Nasca, C. (2015) Mechanisms of stress in the brain. Nature Neuroscience 18(10):1353-63. Available at: http://www.ncbi.nlm.nih.gov/pubmed/ 26404710. [RH]

McGaugh, J. L. (2000). Memory: A century of consolidation. Science 287:248-51. [aMM]

McGaugh, J. L. (2002) Memory consolidation and the amygdala: A systems perspective. Trends in Neuroscience 25(9):456-61. [BR]

McGaugh, J. L. (2004) The amygdala modulates the consolidation of memories of emotionally arousing experiences. Annual Review of Neuroscience 27:1-28. [aMM]

McGaugh, J. L. (2013) Making lasting memories: Remembering the significant. Proceedings of the National Academy of Sciences of the United States of America 110(Suppl. 2):10402-407. doi: 10.1073/pnas.1301209110. [aMM, AB]

McGaughy, J., Ross, R. S. \& Eichenbaum, H. (2008) Noradrenergic, but not cholinergic, deafferentation of prefrontal cortex impairs attentional set-shifting. Neuroscience 153(1):63-71. Available at: http://doi.org/10.1016/j.neuroscience. 2008.01.064. [SB]

McHugh, T. J., Jones, M. W., Quinn, J. J., Balthasar, N., Coppari, R., Elmquist, J. K., Lowell, B. B., Fanselow, M. S., Wilson, M. A. \& Tonegawa, S. (2007) Dentate gyrus NMDA receptors mediate rapid pattern separation in the hippocampal network. Science 317:94-99. [CH]

McIntyre, C. K., Hatfield, T. \& McGaugh, J. L. (2002) Amygdala norepinephrine levels after training predict inhibitory avoidance retention performance in rats. European Journal of Neuroscience 16(7):1223-26. [aMM, MR]

McIntyre, C. K., McGaugh, J. L. \& Williams, C. L. (2012) Interacting brain systems modulate memory consolidation. Neuroscience and Biobehavioral Reviews 36:1750-62. [aMM]

McIntyre, C. K., Miyashita, T., Setlow, B., Marjon, K. D., Steward, O., Guzowski, J F. \& McGaugh, J. L. (2005) Memory-influencing intra-basolateral amygdala drug infusions modulate expression of Arc protein in the hippocampus. Proceedings of the National Academy of Sciences of the United States of America 102(30):10718-23. [BR, rMM]

McLean, J. \& Waterhouse, B. D. (1994) Noradrenergic modulation of cat area 17 neuronal responses to moving stimuli. Brain Research 667:83-97. [QG]

McNaughton, B. L. \& Morris, R. G. M. (1987) Hippocampal synaptic enhancement and information storage within a distributed memory system. Trends in Neurosciences $10: 408-15$. $[\mathrm{CH}]$

McNaughton, N. \& Corr, P. J. (2004) A two-dimensional neuropsychology of defense: Fear/anxiety and defensive distance. Neuroscience and Biobehavioral Reviews 28(3):285-305. [HM]

McReynolds, J. R., Anderson, K. M., Donowho, K. M. \& McIntyre, C. K. (2014) Noradrenergic actions in the basolateral complex of the amygdala modulate Arc expression in hippocampal synapses and consolidation of aversive and nonaversive memory. Neurobiology of Learning and Memory 115:49-57. Available at: http://dx.doi.org/10.1016/j.nlm.2014.08.016. [rMM]

Meldrum, B. S. (2000) Glutamate as a neurotransmitter in the brain: Review of physiology and pathology. The Journal of Nutrition 130(4):1007S-15S. [aMM]

Menon, V. \& Uddin, L. Q. (2010) Saliency, switching, attention and control: A network model of insula function. Brain Structure and Function 214(5/6):65567. doi: 10.1007/s00429-010-0262-0. [aMM]

Merz, C. J., Tabbert, K., Schweckendiek, J., Klucken, T., Vaitl, D., Stark, R. \& Wolf, O. T. (2010) Investigating the impact of sex and cortisol on implicit fear conditioning with fMRI. Psychoneuroendocrinology 35(1):33-46. Available at: http://www.ncbi.nlm.nih.gov/pubmed/19683399. [RH]
Meyer, K. (2015) The role of dendritic signaling in the anesthetic suppression of consciousness. Anesthesiology 122:1415-31. [MEL]

Mihov, Y., Mayer, S., Musshoff, F., Maier, W., Kendrick, K. M. \& Hurlemann, R. (2010) Facilitation of learning by social-emotional feedback in humans is betanoradrenergic-dependent. Neuropsychologia 48(10):3168-72. Available at: http://www.ncbi.nlm.nih.gov/pubmed/20457167. [RH]

Miranda, M. I. \& McGaugh, J. L. (2004) Enhancement of inhibitory avoidance and conditioned taste aversion memory with insular cortex infusions of 8-Br-cAMP Involvement of the basolateral amygdala. Learning and Memory 11(3):312-17. doi: $10.1101 / 1 \mathrm{~lm} .72804 . \quad$ [aMM]

Misu, Y. \& Kubo, T. (1986) Presynaptic $\beta$-adrenoceptors. Medicinal Research Reviews 6(2):197-225. [aMM]

Mobley, P. \& Greengard, P. (1985) Evidence for widespread effects of noradrenaline on axon terminals in the rat frontal cortex. Proceedings of the National Academy of Sciences of the United States of America 82(3):945-47. [aMM]

Moghaddam, B., Bolinao, M. L., Stein-Behrens, B. \& Sapolsky, R. (1994) Glucocortcoids mediate the stress-induced extracellular accumulation of glutamate. Brain Research 655(1):251-54. [rMM]

Mohanty, A., Gitelman, D. R., Small, D. M. \& Mesulam, M. M. (2008) The spatial attention network interacts with limbic and monoaminergic systems to modulate motivation-induced attention shifts. Cerebral Cortex 18(11):2604-13. doi: 10.1093/cercor/bhn021. [aMM]

Moises, H. C., Waterhouse, B. D. \& Woodward, D. J. (1983) Locus coeruleus stimulation potentiates local inhibitory processes in rat cerebellum. Brain Research Bulletin 10:795-804. [RN]

Moises, H. C., Woodward, D. J., Hoffer, B. J. \& Freedman, R. (1979) Interactions of norepinephrine with Purkinje cell responses to putative amino acid neurotransmitters applied by microiontophoresis. Experimental Neurology 64:493-515. [EE]

Moncada, D., Ballarini, F., Martinez, M. C., Frey, J. U. \& Viola, H. (2011) Identification of transmitter systems and learning tag molecules involved in behavioral tagging during memory formation. Proceedings of the National Academy of Sciences of the United States of America 108(31):12931-36. doi: 10.1073/ pnas.1104495108. [arMM, MR]

Moncada, D. \& Viola, H. (2007) Induction of long-term memory by exposure to novelty requires protein synthesis: Evidence for a behavioral tagging. The Journal of Neuroscience 27(28):7476-81. doi: 10.1523/jneurosci.108307.2007. [aMM, MR]

Montagrin, A., Brosch, T. \& Sander, D. (2013) Goal conduciveness as a key determinant of memory facilitation. Emotion 13(4):622-28. doi: 10.1037/ a0033066. [arMM, AM]

Monti, J. M., Torterolo, P. \& Lagos, P. (2013) Melanin-concentrating hormone control of sleep-wake behavior. Sleep Medicine Reviews 17:293-98. doi: 10.1016/j.smrv.2012.10.002. [AB]

Montoya, E. R., Bos, P. A., Terburg, D., Rosenberger, L. A. \& van Honk, J. (2014) Cortisol administration induces global down-regulation of the brain's reward circuitry. Psychoneuroendocrinology 47:31-42. Available at: http://www.ncbi. nlm.nih.gov/pubmed/25001954. [RH]

Moray, N. (1959) Attention in dichotic listening: Affective cues and the influence of instructions. Quarterly Journal of Experimental Psychology 11(1):56-60. [aMM]

Moriceau, S. \& Sullivan, R. M. (2004) Unique neural circuitry for neonatal olfactory learning. The Journal of Neuroscience 24(5):1182-89. doi: 10.1523/jneurosci.4578-03.2004. [RG, RMT ]

Morilak, D. A., Fornal, C. \& Jacobs, B. L. (1986) Single unit activity of noradrenergic neurons in locus coeruleus and serotonergic neurons in the nucleus raphe dorsalis of freely moving cats in relation to the cardiac cycle. Brain Research 399 (2):262-70. [HDC, rMM]

Morimoto, M., Morita, N., Ozawa, H., Yokoyama, K. \& Kawata, M. (1996) Distribution of glucocorticoid receptor immunoreactivity and mRNA in the rat brain: An immunohistochemical and in situ hybridization study. Neuroscience Research 26(3):235-69. [rMM]

Morrison, J. H. \& Foote, S. L. (1986) Noradrenergic and serotoninergic innervation of cortical, thalamic, and tectal visual structures in Old and New World monkeys. Journal of Comparative Neurology 243(1):117-38. doi: 10.1002/ cne.902430110. [aMM]

Most, S. B., Chun, M. M., Widders, D. M. \& Zald, D. H. (2005) Attentional rubbernecking: Cognitive control and personality in emotion-induced blindness. Psychonomic Bulletin and Review 12(4):654-61. [aMM]

Mouradian, R. D., Seller, F. M. \& Waterhouse, B. D. (1991) Noradrenergic potentiation of excitatory transmitter action in cerebrocortical slices: Evidence of mediation by an alphal-receptor-linked second messenger pathway. Brain Research 546:83-95. [CGA, RN]

Mouras, H., Lelard, T., Ahmadi, S., Godefroy, O. \& Krystkowiak, P. (2015) Freezing behavior as a response to sexual visual stimuli as demonstrated by posturography. PLoS ONE 10(5):e0127097. [HM]

Muller, A., Joseph, V., Slesinger, P. A. \& Kleinfeld, D. (2014) Cell-based reporters reveal in vivo dynamics of dopamine and norepinephrine release in murine cortex. Nature Methods 11(12):1245-52. [rMM] 
Murase, S., Inui, K. \& Nosaka, S. (1994) Baroreceptor inhibition of the locus coeruleus noradrenergic neurons Neuroscience 61:635-43. [HDC]

Murphy, P. R., O’Connell, R. G., O’Sullivan, M., Robertson, I. H. \& Balsters, J. H. (2014) Pupil diameter covaries with BOLD activity in human locus coeruleus. Human Brain Mapping 35(8):4140-54. [aMM]

Murray, B. D., Holland, A. C. \& Kensinger, E. A. (2013) Episodic memory and emotion. In: Handbook of cognition and emotion, ed. M. D. Robinson, E. Watkins \& E. Harmon-Jones, pp. 156-75. Guilford Press. [aMM]

Murre, J., Phaf, R. \& Wolters, G. (1992) CALM: Categorizing and learning module. Neural Networks 5:55-82. [RHP]

Murty, V. P., Ritchey, M., Adcock, R. A. \& LaBar, K. S. (2010) fMRI studies of successful emotional memory encoding: A quantitative meta-analysis. Neuropsychologia 48(12):3459-69. doi: 10.1016/j.neuropsychologia.2010.07.030. [aMM]

Murugaiah, K. D. \& O’Donnell, J. M. (1995a) Beta adrenergic receptors facilitate norepinephrine release from rat hypothalamic and hippocampal slices. Research Communications in Molecular Pathology and Pharmacology 90(2):179-90. [aMM]

Murugaiah, K. D. \& O'Donnell, J. M. (1995b) Facilitation of noradrenaline release from rat brain slices by $\beta$-adrenoceptors. Naunyn-Schmiedeberg's Archives of Pharmacology 351(5):483-90. [aMM]

Muth, C. \& Carbon, C. C. (2013) The aesthetic aha: On the pleasure of having insights into Gestalt. Acta Psychologica 144(1):25-30. [C-CC]

Muth, C., Pepperell, R. \& Carbon, C. C. (2013) Give me Gestalt! Preference for cubist artworks revealing high detectability of objects. Leonardo 46(5):48889. [C-CC]

Muth, C., Raab, M. H. \& Carbon, C. C. (2015) The stream of experience when watching artistic movies. Dynamic aesthetic effects revealed by the continuous evaluation procedure (CEP). Frontiers in Psychology 6(365):1-13. [C-CC]

Nabavi, S., Fox, R., Proulx, C. D., Lin, J. Y., Tsien, R. Y. \& Malinow, R. (2014) Engineering a memory with LTD and LTP. Nature 511(7509):348-52. doi: 10.1038/nature13294. [aMM]

Nai, Q., Dong, H.-W., Hayar, A., Linster, C. \& Ennis, M. (2009) Noradrenergic regulation of GABAergic inhibition of main olfactory bulb mitral cells varies as a function of concentration and receptor subtype. Journal of Neurophysiology 101(5):2472-84. [aMM]

Nai, Q., Dong, H. W., Linster, C. \& Ennis, M. (2010) Activation of alphal and alpha2 noradrenergic receptors exert opposing effects on excitability of main olfactory bulb granule cells. Neuroscience 169:882-92. [RN]

Nakamura, S., Kimura, F. \& Sakaguchi, T. (1987) Postnatal development of electrical activity in the locus ceruleus. Journal of Neurophysiology 58(3):510-24. [RG]

Nakamura, S. \& Sakaguchi, T. (1990). Development and plasticity of the locus coeruleus: A review of recent physiological and pharmacological experimentation. Progress in Neurobiology 34(6):505-26. Available at: http://dx.doi.org/10. 1016/0301-0082(90)90018-C. [RG]

Nassar, M. R., Bruckner, R., Gold, J. I., Li, S., Heekeren, H. R. \& Eppinger, B. (2016)Age differences in learning emerge from an insufficient representation of uncertainty in older adults. Nature Communications. [MRN]

Nassar, M. R., Rumsey, K. M., Wilson, R. C., Parikh, K., Heasly, B. \& Gold, J. I. (2012) Rational regulation of learning dynamics by pupil-linked arousal systems. Nature Neuroscience 15(7):1040-46. Available at: http://doi.org/10.1038/nn. 3130. [SB, MRN]

Nelson, M. F., Zaczek, R. \& Coyle, J. T. (1980) Effects of sustained seizures produced by intrahippocampal injection of kainic acid on noradrenergic neurons: Evidence for local control of norepinephrine release. Journal of Pharmacology and Experimental Therapeutics 214(3):694-702. [arMM]

Neuman, R. \& Harley, C. (1983) Long-lasting potentiation of the dentate gyrus population spike by norepinephrine. Brain Research 273:162-65. [CH]

Nicholson, E. L., Bryant, R. A. \& Felmingham, K. L. (2014) Interaction of noradrenaline and cortisol predicts negative intrusive memories in posttraumatic stress disorder. Neurobiology of Learning and Memory 112:204-11. Available at: http://www.ncbi.nlm.nih.gov/pubmed/24296460. [RH]

Nicoll, R. A. (1988) The coupling of neurotransmitter receptors to ion channels in the brain. Science 241(4865):545-51. [aMM]

Nielsen, S. E., Barber, S. J., Chai, A., Clewett, D. V. \& Mather, M. (2015) Sympathetic arousal increases a negative memory bias in young women with low sex hormone levels. Psychoneuroendocrinology 62:96-106. [rMM]

Nielsen, S. E., Chai, A. \& Mather, M. (in preparation) Sympathetic arousal enhances memory for negative stimuli in older women not taking hormone replacement therapy. [rMM]

Nielsen, S. E. \& Mather, M. (2015) Comparison of two isometric handgrip protocols on sympathetic arousal in women. Physiology and Behavior 142:5-13. $[\mathrm{rMM}]$

Nielson, K. A. \& Powless, M. (2007) Positive and negative sources of emotional arousal enhance long-term word-list retention when induced as long as $30 \mathrm{~min}$ after learning. Neurobiology of Learning and Memory 88(1):40-47. doi: 10.1016/j.nlm.2007.03.005. [aMM]
Nieuwenhuis, S., Aston-Jones, G. \& Cohen, J. D. (2005a) Decision making, the P3, and the locus coeruleus-norepinephrine system. Psychological Bulletin 131 (4):510-32. [rMM]

Nieuwenhuis, S., De Geus, E. J. \& Aston-Jones, G. (2011) The anatomical and functional relationship between the P3 and autonomic components of the orienting response. Psychophysiology 48:162-75. [MRN]

Nieuwenhuis, S. \& de Kleijn, R. (2013) The impact of alertness on cognitive control. Journal of Experimental Psychology: Human Perception and Performance 39 (6): 1797. [CMW]

Nieuwenhuis, S., Gilzenrat, M. S., Holmes, B. D. \& Cohen, J. D. (2005b) The role of the locus coeruleus in mediating the attentional blink: A neurocomputational theory. Journal of Experimental Psychology: General 134:291-307. [CMW]

Nitz, D. \& McNaughton, B. (2004) Differential modulation of CAl and dentate gyrus interneurons during exploration of novel environments. Journal of Neurophysiology 91:863-72. [CH]

Niu, Y., Todd, R. \& Anderson, A. K. (2012) Affective salience can reverse the effects of stimulus-driven salience on eye movements in complex scenes. Frontiers in Psychology 3:336. doi: 10.3389/fpsyg.2012.00336. [aMM]

Nomura, S., Bouhadana, M., Morel, C., Faure, P., Cauli, B., Lambolez, B. \& Hepp, R. (2014) Noradrenalin and dopamine receptors both control cAMP-PKA signaling throughout the cerebral cortex. Frontiers in Cellular Neuroscience 8:247. [aMM]

Oades, R. D. (1985) The role of noradrenaline in tuning and dopamine in switching between signals in the CNS. Neuroscience and Biobehavioral Reviews 9(2):26182. $[\mathrm{aMM}]$

O'Dell, T. J., Connor, S. A., Gelinas, J. N. \& Nguyen, P. V. (2010) Viagra for your synapses: Enhancement of hippocampal long-term potentiation by activation of beta-adrenergic receptors. Cellular Signalling 22(5):728-36. doi: 10.1016/j. cellsig.2009.12.004. [arMM]

O’Donnell, J., Zeppenfeld, D., McConnell, E., Pena, S. \& Nedergaard, M. (2012) Norepinephrine: A neuromodulator that boosts the function of multiple cell types to optimize CNS performance. Neurochemical Research 37(11):2496512. doi: 10.1007/s11064-012-0818-x. [aMM]

Öhman, A., Flykt, A. \& Esteves, F. (2001) Emotion drives attention: Detecting the snake in the grass. Journal of Experimental Psychology: General 130(3):46678. $[\mathrm{aMM}]$

Oke, A., Keller, R., Mefford, I. \& Adams, R. N. (1978) Lateralization of norepinephrine in human thalamus. Science 200(4348):1411-13. [aMM]

O'Keefe, J. \& Dostrovsky, J. (1971) The hippocampus as a spatial map: Preliminary evidence from unit activity in the freely-moving rat. Brain Research 34:171-75. [SJS]

Okubo, Y. \& Iino, M. (2011) Visualization of glutamate as a volume transmitter. The Journal of Physiology 589(3):481-88. [aMM]

Okubo, Y., Sekiya, H., Namiki, S., Sakamoto, H., Iinuma, S., Yamasaki, M., Watanabe, M., Hirose, K. \& Iino, M. (2010) Imaging extrasynaptic glutamate dynamics in the brain. Proceedings of the National Academy of Sciences of the United States of America 107(14):6526-31. [arMM]

Olpe, H. R., Glatt, A., Laszlo, J. \& Schellenberg, A. (1980) Some electrophysiological and pharmacological properties of the cortical, noradrenergic projection of the locus coeruleus in the rat. Brain Research 186:9-19. [QG]

Olshausen, B. A., Anderson, C. H. \& Van Essen, D. C. (1993) A neurobiological model of visual attention and invariant pattern recognition based on dynamic routing of information. The Journal of Neuroscience 13:4700-19. [CH]

Onur, O. A., Walter, H., Schlaepfer, T. E., Rehme, A. K., Schmidt, C., Keysers, C. Maier, W. \& Hurlemann, R. (2009) Noradrenergic enhancement of amygdala responses to fear. Social Cognitive and Affective Neuroscience 4(2):119-26. Available at: http://www.ncbi.nlm.nih.gov/pubmed/19246474. [RH]

O'Reilly, R. C. \& McClelland, J. L. (1994) Hippocampal conjunctive encoding, storage, and recall: Avoiding a trade-off. Hippocampus 4:661-82. [CH]

Pacak, K. \& Palkovits, M. (2001) Stressor specificity of central neuroendocrine responses: Implications for stress-related disorders. Endocrine Reviews 22 (4):502-48. [aMM]

Padmala, S., Lim, S.-L. \& Pessoa, L. (2010) Pulvinar and affective significance: Responses track moment-to-moment stimulus visibility. Frontiers in Human Neuroscience 4. doi: 10.3389/fnhum.2010.00064. [aMM]

Padmala, S. \& Pessoa, L. (2008) Affective learning enhances visual detection and responses in primary visual cortex. Journal of Neuroscience 28(24):6202-10. doi: 10.1523/jneurosci.1233-08.2008. [aMM]

Palamarchouk, V. S., Zhang, J.-J., Zhou, G., Swiergiel, A. H. \& Dunn, A. J. (2000) Hippocampal norepinephrine-like voltammetric responses following infusion of corticotropin-releasing factor into the locus coeruleus. Brain Research Bulletin 51(4):319-26. [aMM]

Palmer, L. M., Shai, A. S., Reeve, J. E., Andersen, H. L., Paulsen, O. \& Larkum, M E. (2014) NMDA spikes enhance action potential generation during sensory input. Nature Neuroscience 17:383-90. [MEL]

Panksepp, J. (2007) Neurologizing the psychology of affects: How appraisal-based constructivism and basic emotion theory can coexist. Perspectives on Psychological Science 2(3):281-96. [TDH] 
Panksepp, J. \& Biven, L. (2012) The archaeology of mind: Neuroevolutionary origins of human emotions (Norton series on interpersonal neurobiology). WW Norton. [HM]

Parikh, V., Man, K., Decker, M. W. \& Sarter, M. (2008) Glutamatergic contributions to nicotinic acetylcholine receptor agonist-evoked cholinergic transients in the prefrontal cortex. Journal of Neuroscience 28:3769-80. doi: 10.1523/JNEUROSCI.5251-07.2008. [AB]

Park, H. D., Correia, S., Ducorps, A. \& Tallon-Baudry, C. (2014) Spontaneous fluctuations in neural responses to heartbeats predict visual detection. Nature Neuroscience 17:612-18. [HDC]

Parkhurst, D., Law, K. \& Niebur, E. (2002) Modeling the role of salience in the allocation of overt visual attention. Vision Research 42(1):107-23. [aMM]

Parpura, V. \& Haydon, P. G. (2000) Physiological astrocytic calcium levels stimulate glutamate release to modulate adjacent neurons. Proceedings of the National Academy of Sciences of the United States of America 97(15):8629-34. [aMM]

Parrott, W. G. \& Sabini, J. (1990) Mood and memory under natural conditions: Evidence for mood incongruent recall. Journal of Personality and Social Psychology 59:321-36. [KK]

Pastor, M. C., Bradley, M. M., Löw, A., Versace, F., Moltó, J. \& Lang, P. J. (2008) Affective picture perception: Emotion, context, and the late positive potential. Brain Research 1189:145-51. [DT]

Patin, A. \& Hurlemann, R. (2011) Modulating amygdala responses to emotion: Evidence from pharmacological fMRI. Neuropsychologia 49(4):706-17. Available at: http://www.ncbi.nlm.nih.gov/pubmed/20933529. [RH]

Paukert, M., Agarwal, A., Cha, J., Doze, V. A., Kang, J. U. \& Bergles, D. E. (2014) Norepinephrine controls astroglial responsiveness to local circuit activity. Neuron 82(6):1263-70. [aMM]

Pawlak, V., Wickens, J. R., Kirkwood, A. \& Kerr, J. N. (2010) Timing is not every thing: Neuromodulation opens the STDP gate. Frontiers in Synaptic Neuroscience $2: 146 . \quad[\mathrm{aMM}]$

Payne, J. D., Chambers, A. M. \& Kensinger, E. A. (2012) Sleep promotes lasting changes in selective memory for emotional scenes. Frontiers in Integrative Neuroscience 6:108. doi: 10.3389/fnint.2012.00108. [aMM]

Payne, J. D., Nadel, L., Allen, J. J. B., Thomas, K. \& Jacobs, J. (2002) The effects of experimentally induced stress on false recognition. Memory 10:1-6. [JRH]

Payne, J. D., Stickgold, R., Swanberg, K. \& Kensinger, E. A. (2008) Sleep preferentially enhances memory for emotional components of scenes. Psychological Science 19(8):781. doi: 10.1111/j.1467-9280.2008.02157.x. [aMM]

Paz, R., Bauer, E. P. \& Paré, D. (2008) Theta synchronizes the activity of medial prefrontal neurons during learning. Learning and Memory 15(7):524-31. $[\mathrm{aMM}]$

Pellerin, L. \& Magistretti, P. J. (2012) Sweet sixteen for ANLS. Journal of Cerebral Blood Flow and Metabolism 32(7):1152-66. [aMM]

Pessoa, L. (2008) On the relationship between emotion and cognition. Nature 9:14858. [RHP]

Pessoa, L. (2009) How do emotion and motivation direct executive control? Trends in Cognitive Sciences 13(4):160-66. [aMM]

Pessoa, L. (2013) The cognitive-emotional brain: From interactions to integration: MIT Press. [aMM]

Pessoa, L. \& Adolphs, R. (2010) Emotion processing and the amygdala: From a "low road" to "many roads" of evaluating biological significance. Nature Reviews Neuroscience 11(11):773-83. [aMM]

Petersen, S. E. \& Posner, M. I. (2012) The attention system of the human brain: 20 years after. Annual Review of Neuroscience 35:73-89. doi: 10.1146/annurevneuro-062111-150525. [FF-S]

Petralia, R., Yokotani, N. \& Wenthold, R. (1994) Light and electron microscope distribution of the NMDA receptor subunit NMDAR1 in the rat nervous system using a selective anti-peptide antibody. Journal of Neuroscience 14 (2):667-96. [aMM]

Petroff, O. A. (2002) Book review: GABA and glutamate in the human brain. The Neuroscientist 8(6):562-73. [aMM]

Pfaff, D. W. (2006) Brain arousal and information theory: Harvard University Press. [rMM]

Phaf, R. H. \& Rotteveel, M. (2005) Affective modulation of recognition bias. Emotion 5:309-18. [RHP]

Phaf, R. H. \& Rotteveel, M. (2012) Affective monitoring: A generic mechanism for affect elicitation. Frontiers in Psychology 3:47. doi: 10.3389/ fpsyg.2012.00047. [RHP]

Phaf, R. H., Van der Heijden, A. H. C. \& Hudson, P. T. (1990) SLAM: A connectionist model for attention in visual selection tasks. Cognitive Psychology 22:273-341. [RHP]

Phan, K. L., Wager, T., Taylor, S. F. \& Liberzon, I. (2002) Functional neuroanatomy of emotion: A meta-analysis of emotion activation studies in PET and fMRI. NeuroImage 16:331-48. [aMM]

Phelps, E. A. (2004) Human emotion and memory: Interactions of the amygdala and hippocampal complex. Current Opinion in Neurobiology 14(2):198-202. [aMM]
Phelps, E. A., Ling, S. \& Carrasco, M. (2006) Emotion facilitates perception and potentiates the perceptual benefits of attention. Psychological Science 17 (4):292-99. doi: 10.1111/j.1467-9280.2006.01701.x. [aMM]

Phillips, W. A. (2015) Cognitive functions of intracellular mechanisms for contextual amplification. Brain and Cognition. Available at: http://dx.doi.org/10.1016/j. bandc.2015.09.005. [MEL, rMM]

Phillips, W. A., Clark, A. \& Silverstein, S. M. (2015) On the functions, mechanisms, and malfunctions of intracortical contextual modulation. Neuroscience and Biobehavioral Reviews 52:1-20. doi: 10.1016/j.neubiorev.2015.02.010. [MEL]

Piech, R. M., McHugo, M., Smith, S. D., Dukic, M. S., Van Der Meer, J., AbouKhalil, B., Most, S. B. \& Zald, D. H. (2011) Attentional capture by emotional stimuli is preserved in patients with amygdala lesions. Neuropsychologia 49 (12):3314-19. [rMM]

Piech, R. M., McHugo, M., Smith, S. D., Dukic, M. S., Van Der Meer, J., AbouKhalil, B. \& Zald, D. H. (2010) Fear-enhanced visual search persists after amygdala lesions. Neuropsychologia 48(12):3430-35. [rMM]

Pittaluga, A. \& Raiteri, M. (1990) Release-enhancing glycine-dependent presynaptic NMDA receptors exist on noradrenergic terminals of hippocampus. European Journal of Pharmacology 191(2):231-34. [arMM, CH]

Pittaluga, A. \& Raiteri, M. (1992) N-Methyl-D-aspartic acid (NMDA) and nonNMDA receptors regulating hippocampal norepinephrine release: I. Location on axon terminals and pharmacological characterization. Journal of Pharmacology and Experimental Therapeutics 260(1):232-37. [aMM]

Pittaluga, A., Fedele, E., Risiglione, C. \& Raiteri, M. (1993) Age-related decrease of the NMDA receptor-mediated noradrenaline release in rat hippocampus and partial restoration by D-cycloserine. European Journal of Pharmacology 231 (1):129-34. [aMM]

Pittaluga, A., Pattarini, R., Andrioli, G. C., Viola, C., Munari, C. \& Raiteri, M. (1999 Activity of putative cognition enhancers in kynurenate test performed with human neocortex slices. Journal of Pharmacology and Experimental Therapeutics 290(1):423-28. [aMM]

Poe, G. \& Sara, S. J. (2014) Locus coeruleus activity time-locked to hippocampal rhythms during sleep. Program No. 652.16 2014 Neuroscience Meeting Planner, Washington, DC: Society for Neuroscience. Online [SJS]

Polack, P.-O., Friedman, J. \& Golshani, P. (2013) Cellular mechanisms of brain statedependent gain modulation in visual cortex. Nature Neuroscience 16(9):133139. [aMM]

Ponzio, A. \& Mather, M. (2014) Hearing something emotional affects memory for what was just seen: How arousal amplifies trade-off effects in memory consolidation. Emotion 14:1137-42. [arMM]

Pool, E., Brosch, T., Delplanque, S. \& Sander, D. (2015) Attentional bias for positive emotional stimuli: A meta-analytic investigation. Psychological Bulletin 142 (1):79-106. [AM, rMM]

Popoli, M., Yan, Z., McEwen, B. S. \& Sanacora, G. (2012) The stressed synapse: The impact of stress and glucocorticoids on glutamate transmission. Nature Reviews Neuroscience 13(1):22-37. [rMM]

Pourtois, G., Schettino, A. \& Vuilleumier, P. (2013) Brain mechanisms for emotional influences on perception and attention: What is magic and what is not. Biological Psychology 92(3):492-512. [aMM]

Preuschoff, K. (2011) Pupil dilation signals surprise: Evidence for noradrenaline's role in decision making. Frontiers in Neuroscience 5:Article 115. doi: 10.3389/ fnins.2011.00115/abstract. [MRN]

Preuschoff, K., Marius’t Hart, B. \& Einhäuser, W. (2011) Pupil dilation signals surprise: Evidence for noradrenaline's role in decision making. Frontiers in Neuroscience 5:Article 115. Available at: http://doi.org/10.3389/fnins.2011. 00115. [SB, rMM]

Price, J. L. \& Amaral, D. G. (1981) An autoradiographic study of the projections of the central nucleus of the monkey amygdala. The Journal of Neuroscience 1 (11):1242-59. [aMM]

Proulx, E., Suri, D., Heximer, S. P., Vaidya, V. A. \& Lambe, E. K. (2014) Early stress prevents the potentiation of muscarinic excitation by calcium release in adult prefrontal cortex. Biological Psychiatry 76:315-23. doi: 10.1016/j.biopsych.2013.10.017. [AB]

Przybyslawski, J., Roullet, P. \& Sara, S. J. (1999) Attenuation of emotional and nonemotional memories after their reactivation: Role of $\beta$ adrenergic receptors. The Journal of Neuroscience 19(15):6623-28. [aMM]

Ptak, R. (2012) The frontoparietal attention network of the human brain action, saliency, and a priority map of the environment. The Neuroscientist 18(5):502 15. $[\mathrm{aMM}]$

Purushothaman, G., Marion, R., Li, K. \& Casagrande, V. A. (2012) Gating and control of primary visual cortex by pulvinar. Nature Neuroscience 15(6):90512. [aMM]

Quirarte, G. L., Roozendaal, B. \& McGaugh, J. L. (1997) Glucocorticoid enhancement of memory storage involves noradrenergic activation in the basolateral amygdala. Proceedings of the National Academy of Sciences of the United States of America 94(25):14048-53. [BR] 
Rainville, P., Duncan, G. H., Price, D. D., Carrier, B. \& Bushnell, M. C. (1997) Pain affect encoded in human anterior cingulate but not somatosensory cortex. Science 277(5328):968-71. doi: 10.2307/2892916. [aMM]

Raio, C. M., Orederu, T. a, Palazzolo, L., Shurick, A. A. \& Phelps, E. A. (2013) Cognitive emotion regulation fails the stress test. Proceedings of the National Academy of Sciences of the United States of America 110(37):15139-44. [TDH]

Raiteri, M., Garrone, B. \& Pittaluga, A. (1992) N-Methyl-D-aspartic acid (NMDA) and non-NMDA receptors regulating hippocampal norepinephrine release. II. Evidence for functional cooperation and for coexistence on the same axon terminal. Journal of Pharmacology and Experimental Therapeutics 260:23842. $[\mathrm{CH}]$

Rajkowski, J., Kubiak, P. \& Aston-Jones, G. (1994) Locus coeruleus activity in monkey: Phasic and tonic changes are associated with altered vigilance. Brain Research Bulletin 35(5/6):607-16. Available at: http://dx.doi.org/10.1016/0361 9230(94)90175-9. [RG]

Rajkowski, J., Majczynski, H., Clayton, E. \& Aston-Jones, G. (2004) Activation of monkey locus coeruleus neurons varies with difficulty and performance in a target detection task. Journal of Neurophysiology 92(1):361-71. [RN, SJS]

Ramos, B. P. \& Arnsten, A. F. T. (2007) Adrenergic pharmacology and cognition: Focus on the prefrontal cortex. Pharmacology and Therapeutics 113(3):52336. [arMM]

Ramos, B. P., Stark, D., Verduzco, L., van Dyck, C. H. \& Arnsten, A. F. (2006) $\alpha 2$ Aadrenoceptor stimulation improves prefrontal cortical regulation of behavior through inhibition of cAMP signaling in aging animals. Learning and Memory 13(6):770-76. [aMM, RMT]

Rangel, S. \& Leon, M. (1995) Early odor preference training increases olfactory bulb norepinephrine. Developmental Brain Research 85(2):187-91. Available at: http://dx.doi.org/10.1016/0165-3806(94)00211-H. [aMM, RG]

Rao, R. P. N. \& Ballard, D. H. (1999) Predictive coding in the visual cortex: A functional interpretation of some extra-classical receptive-field effects. Nature Neuroscience 2:79-97. doi: 10.1038/4580. [FF-S]

Rasch, B. \& Born, J. (2013) About sleep's role in memory. Physiological Reviews 93:681-766. doi: 10.1152/physrev.00032.2012. [AB]

Rasch, B., Spalek, K., Buholzer, S., Luechinger, R., Boesiger, P., Papassotiropoulos, A. \& Quervain, D. J. F. d. (2009) A genetic variation of the noradrenergic system is related to differential amygdala activation during encoding of emotional memories. Proceedings of the National Academy of Sciences of the United States of America 106(45):19191-96. doi: 10.2307/25593165. [aMM, RMT]

Rauchs, G., Feyers, D., Landeau, B., Bastin, C., Luxen, A., Maquet, P. \& Collette, F. (2011) Sleep contributes to the strengthening of some memories over others, depending on hippocampal activity at learning. The Journal of Neuroscience 31 (7):2563-68. doi: 10.1523/jneurosci.3972-10.2011. [aMM]

Raymond, J. E., Fenske, M. J. \& Tavassoli, N. T. (2003) Selective attention determines emotional responses to novel visual stimuli. Psychological Science 14:537-42. [RHP]

Redondo, R. L. \& Morris, R. G. (2011) Making memories last: The synaptic tagging and capture hypothesis. Nature Reviews Neuroscience 12(1):17-30. [MR, $\mathrm{rMM}]$

Reed, A. E., Chan, L. \& Mikels, J. A. (2014) Meta-analysis of the age-related positivity effect: Age differences in preferences for positive over negative information. Psychology and Aging 29:1-15. [KK]

Rei, D., Mason, X., Seo, J., Gräff, J., Rudenko, A., Wang, J., Rueda, R., Siegert, S., Cho, S., Canter, R. G., Mungenast, A. E., Deisseroth, K. \& Tsai, L. H. (2015) Basolateral amygdala bidirectionally modulates stress-induced hippocampal learning and memory deficits through a p25/Cdk5-dependent pathway. Proceedings of the National Academy of Sciences of the United States of America 112(23):7291-96. doi: 10.1073/pnas.1415845112. [rMM]

Reicher, G. M., Snyder, C. R. \& Richards, J. T. (1976) Familiarity of background characters in visual scanning. Journal of Experimental Psychology: Human Perception and Performance 2(4):522. [aMM]

Reimer, J., Froudarakis, E., Cadwell, C. R., Yatsenko, D., Denfield, G. H. \& Tolias, A. S. (2014) Pupil fluctuations track fast switching of cortical states during quiet wakefulness. Neuron 84(2):355-62. [aMM]

Reisberg, D. \& Heuer, F. (2004) Memory for emotional events. In: Memory and emotion, ed. D. Reisberg \& P. Hertel, pp. 3-41. Oxford University Press. [aMM, AM]

Reynolds, J. H. \& Desimone, R. (2003) Interacting roles of attention and visual salience in V4. Neuron 37(5):853-63. [aMM]

Reynolds, J. H. \& Heeger, D. J. (2009) The normalization model of attention. Neuron 61(2):168-85. [aMM]

Reznikov, L. R., Grillo, C. A., Piroli, G. G., Pasumarthi, R. K., Reagan, L. P. \& Fadel, J. (2007) Acute stress-mediated increases in extracellular glutamate levels in the rat amygdala: Differential effects of antidepressant treatment. European Journal of Neuroscience 25(10):3109-14. [rMM]

Richardson, M. P., Strange, B. A. \& Dolan, R. J. (2004) Encoding of emotional memories depends on amygdala and hippocampus and their interactions. Nature Neuroscience 7(3):278-85. [aMM, BR]
Richter-Levin, G. \& Akirav, I. (2003) Emotional tagging of memory formation - in the search for neural mechanisms. Brain Research Reviews 43(3):247-56. [aMM]

Rinne, A., Birk, A. \& Bünemann, M. (2013) Voltage regulates adrenergic receptor function. Proceedings of the National Academy of Sciences of the United States of America 110(4):1536-41. [aMM]

Ritchey, M., Dolcos, F. \& Cabeza, R. (2008) Role of amygdala connectivity in the persistence of emotional memories over time: An event-related fMRI investigation. Cerebral Cortex 18(11):2494-504. doi: 10.1093/cercor/bhm262. [aMM]

Robertson, S. D., Plummer, N. W., de Marchena, J. \& Jensen, P. (2013) Developmental origins of central norepinephrine neuron diversity. Nature Neuroscience 16(8):1016-23. [aMM]

Robinson, R. B. \& Siegelbaum, S. A. (2003) Hyperpolarization-activated cation currents: From molecules to physiological function. Annual Review of Physiology 65(1):453-80. [aMM]

Roediger, H. L. \& McDermott, K. B. (1995) Creating false memories: Remembering words not presented in lists. Journal of Experimental Psychology: Learning, Memory, and Cognition 21(4):803. [JRH]

Roelofs, K., Hagenaars, M. A. \& Stins, J. (2010) Facing freeze: Social threat induces bodily freeze in humans. Psychological Science 21:1575-81. [HM]

Rogawski, M. A. \& Aghajanian, G. K. (1982) Activation of lateral geniculate neurons by locus coeruleus or dorsal noradrenergic bundle stimulation: Selective blockade by the alphal-adrenoceptor antagonist prazosin. Brain Research 250:31-39. [RN]

Rolls, E. T. (1989) The representation and storage of information in neural networks in the primate cerebral cortex and hippocampus. In: The computing neuron, ed. R. Durbin, C. Miall \& G. Mitchison, pp. 125-59. Addison-Wesley. [CH]

Roozendaal, B., Castello, N. A., Vedana, G., Barsegyan, A. \& McGaugh, J. L. (2008) Noradrenergic activation of the basolateral amygdala modulates consolidation of object recognition memory. Neurobiology of Learning and Memory 90 (3):576-79. doi: 10.1016/j.nlm.2008.06.010. [aMM]

Roozendaal, B. \& McGaugh, J. L. (2011) Memory modulation. Behavioral Neuroscience 125(6):797-824. doi: 10.1037/a0026187. [AM, BR]

Roozendaal, B., Nguyen, B. T., Power, A. E. \& McGaugh, J. L. (1999) Basolateral amygdala noradrenergic influence on the memory-enhancing effect of glucocorticoid receptor activation in the hippocampus. Proceedings of the National Academy of Sciences of the United States of America 96(20):11642-47. [BR]

Rosanova, M. \& Ulrich, D. (2005) Pattern-specific associative long-term potentiation induced by a sleep spindle-related spike train. The Journal of Neuroscience 25 (41):9398-405. doi: 10.1523/jneurosci.2149-05.2005. [aMM]

Rotteveel, M. \& Phaf, R. H. (2007) Mere exposure in reverse: Mood and motion modulate memory bias. Cognition and Emotion 21:1323-46. [RHP]

Russell, J. A. (1989) Measures of emotion. In: Emotion: Theory, research, and experience, ed. R. Plutchik \& H. Kellerman, pp. 83-111. Academic Press. [AM]

Saalmann, Y. B. \& Kastner, S. (2009) Gain control in the visual thalamus during perception and cognition. Current Opinion in Neurobiology 19(4):408-14. [aMM]

Saalmann, Y. B., Pinsk, M. A., Wang, L., Li, X. \& Kastner, S. (2012) The pulvinar regulates information transmission between cortical areas based on attention demands. Science 337(6095):753-56. doi: 10.1126/science.1223082. [aMM]

Sadaghiani, S. \& D’Esposito, M. (2014) Functional characterization of the cinguloopercular network in the maintenance of tonic alertness. Cerebral Cortex. doi: 10.1093/cercor/bhu072 [aMM]

Sahay, A., Scobie, K. N., Hill, A. S., O'Carroll, C. M., Kheirbek, M. A., Burghardt, N. S., Fenton, A. A., Dranovsky, A. \& Hen, R. (2011) Increasing adult hippocampal neurogenesis is sufficient to improve pattern separation. Nature 472 (7344):466-70. [CH]

Sakaki, M., Fryer, K. \& Mather, M. (2014a) Emotion strengthens high priority memory traces but weakens low priority memory traces. Psychological Science 25(2):387-95. doi: 10.1177/0956797613504784 [arMM, CMW]

Sakaki, M., Ycaza-Herrera, A. E. \& Mather, M. (2014b) Association learning for emotional harbinger cues: When do previous emotional associations impair and when do they facilitate subsequent learning of new associations? Emotion 14 (1):115. [rMM]

Saletin, J. M., Goldstein, A. N. \& Walker, M. P. (2011) The role of sleep in directed forgetting and remembering of human memories. Cerebral Cortex 21 (11):2534-41. doi: 10.1093/cercor/bhr034. [aMM]

Salgado, H., Garcia-Oscos, F., Martinolich, L., Hall, S., Restom, R., Tseng, K. Y. \& Atzori, M. (2012a) Pre- and postsynaptic effects of norepinephrine on $\gamma$-aminobutyric acid-mediated synaptic transmission in layer $2 / 3$ of the rat auditory cortex. Synapse 66(1):20-28. [aMM]

Salgado, H., Kohr, G. \& Trevino, M. (2012b) Noradrenergic "tone" determines dichotomous control of cortical spike-timing-dependent plasticity. Scientific Reports 2:7. doi: 417 10.1038/srep00417. [aMM]

Samuels, E. R. \& Szabadi, E. (2008a) Functional neuroanatomy of the noradrenergic locus coeruleus: Its roles in the regulation of arousal and autonomic function. Part I. Principles of functional organisation. Current Neuropharmacology 6 (3):235-53. doi: 10.2174/157015908785777229. [aMM]

Samuels, E. R. \& Szabadi, E. (2008b) Functional neuroanatomy of the noradrenergic locus coeruleus: Its roles in the regulation of arousal and autonomic function. 
References/Mather et al.: Norepinephrine ignites local hotspots of neuronal excitation

Part II. Physiological and pharmacological manipulations and pathological alterations of locus coeruleus activity in humans. Current Neuropharmacology 6 (3):254-85. doi: 10.2174/157015908785777193. [aMM]

Sander, D. (2013) Models of emotion: The affective neuroscience approach. In: The Cambridge handbook of human affective neuroscience, ed. J. L. Armony \& P. Vuilleumier, pp. 5-53. Cambridge University Press. [AM]

Sander, D., Grafman, J. \& Zalla, T. (2003) The human amygdala: An evolved system for relevance detection. Reviews in the Neurosciences 14(4):303-16. [aMM, $\mathrm{AM}$ ]

Sander, D., Grandjean, D. \& Scherer, K. R. (2005) A systems approach to appraisal mechanisms in emotion. Neural Networks 18:317-52. doi: 10.1016/j. neunet.2005.03.001. [AM]

Sandi, C. (2011) Glucocorticoids act on glutamatergic pathways to affect memory processes. Trends in Neurosciences 34(4):165-76. [rMM]

Saper, C. B., Fuller, P. M., Pedersen, N. P., Lu, J. \& Scammel, T. E. (2010) Sleep state switching. Neuron 68:1023-42. doi: 10.1016/j.neuron.2010.11.032. [AB]

Sara, S. J. (2000) Strengthening the shaky trace through retrieval. Nature Reviews Neuroscience 1(3):212-13. Available at: http://doi.org/10.1038/35044575. [SB]

Sara, S. J. (2009) The locus coeruleus and noradrenergic modulation of cognition. Nature Reviews Neuroscience 10(3):211-23. [aMM, RG]

Sara, S. J. (2010) Reactivation, retrieval, replay and reconsolidation in and out of sleep: Connecting the dots. Frontiers in Behavioral Neuroscience 4. doi: 10.3389/fnbeh.2010.00185. [aMM]

Sara, S. J. (2015) Locus coeruleus in time with the making of memories. Current Opinion in Neurobiology 35:87-94. [SJS, rMM]

Sara, S. J. \& Bouret, S. (2012) Orienting and reorienting: The locus coeruleus mediates cognition through arousal. Neuron 76(1):130-41. doi: 10.1016/j. neuron.2012.09.011. [arMM, SB, SJS]

Sara, S. J. \& Segal, M. (1991) Plasticity of sensory responses of locus coeruleus neurons in the behaving rat: Implications for cognition. Progress in Brain Research 88:571-85. [arMM, SJS]

Sato, H., Fox, K. \& Daw, N. W. (1989) Effect of electrical stimulation of locus coeruleus on the activity of neurons in the cat visual cortex. Journal of Neurophysiology 62:946-58. [QG]

Saunders, C. \& Limbird, L. E. (1999) Localization and trafficking of $\alpha_{2}$-adrenergic receptor subtypes in cells and tissues. Pharmacology and Therapeutics 84 (2):193-205. [aMM]

Scharfman, H. E. (1994) Evidence from simultaneous intracellular recordings in rat hippocampal slices that area CA3 pyramidal cells innervate dentate hilar mossy cells. Journal of Neurophysiology 72:2167-80. [CH]

Scharfman, H. E. (1995) Electrophysiological evidence that dentate hilar mossy cells are excitatory and innervate both granule cells and interneurons. Journal of Neurophysiology 74:179-94. [CH]

Scharfman, H. E. \& Myers, C. E. (2012) Hilar mossy cells of the dentate gyrus: A historical perspective. Frontiers in Neural Circuits 6:106. [CH]

Schmidt, S. L., Chew, E. Y., Bennett, D. V., Hammad, M. A. \& Frölich, F. (2013) Differential effects of cholinergic and noradrenergic neuromodulation on spontaneous cortical network dynamics. Neuropharmacology 72:259-73. doi: 10.1016/j.neuropharm.2013.04.045. [AB]

Schoenbaum, G. \& Roesch, M. R. (2005) Orbitofrontal cortex, associative learning, and expectancies. Neuron 47(5):633-36. [aMM]

Schultz, W. \& Dickinson, A. (2000) Neuronal coding of prediction errors. Annual Review in Neuroscience 23:473-500. doi: 10.1146/annurev.neuro.23.1.473. [FF-S]

Schupp, H. T., Flaisch, T., Stockburger, J. \& Junghofer, M. (2006) Emotion and attention: Event-related brain potential studies. Brain 156:31-51. [DT]

Schupp, H. T., Schmälzle, R., Flaisch, T., Weike, A. I. \& Hamm, A. O. (2012) Affective picture processing as a function of preceding picture valence: An ERP analysis. Biological Psychology 91(1):81-87. [DT]

Schwabe, L., Tegenthoff, M., Höffken, O. \& Wolf, O. T. (2012) Simultaneous glucocorticoid and noradrenergic activity disrupts the neural basis of goal-directed action in the human brain. The Journal of Neuroscience 32(30):10146-55. $[\mathrm{rMM}]$

Schwager, S. \& Rothermund, K. (2013) Counter-regulation triggered by emotions: Positive/negative affective states elicit opposite valence biases in affective processing. Cognition and Emotion 27:839-55. [KK]

Schwarze, U., Bingel, U. \& Sommer, T. (2012) Event-related nociceptive arousal enhances memory consolidation for neutral scenes. The Journal of Neuroscience 32(4):1481-87. [aMM]

Sears, R. M., Fink, A. E., Wigestrand, M. B., Farb, C. R., de Lecea, L. \& LeDoux, J E. (2013) Orexin/hypocretin system modulates amygdala-dependent threat learning through the locus coeruleus. Proceedings of the National Academy of Sciences of the United States of America 110(50):20260-65. [aMM]

Seeley, W. W., Menon, V., Schatzberg, A. F., Keller, J., Glover, G. H., Kenna, H., Reiss, A. L. \& Greicius, M. D. (2007) Dissociable intrinsic connectivity networks for salience processing and executive control. The Journal of Neuroscience 27(9):2349-56. [aMM]
Segal, M. \& Bloom, F. E. (1976) The action of norepinephrine in the rat hippocampus: IV. The effects of locus coeruleus stimulation on evoked hippocampal unit activity. Brain Research 107(3):513-25. [aMM]

Segal, S. K. \& Cahill, L. (2009) Endogenous noradrenergic activation and memory for emotional material in men and women. Psychoneuroendocrinology 34 (9):1263-71. doi: 10.1016/j.psyneuen.2009.04.020. [aMM]

Segal, S. K., Stark, S. M., Kattan, D., Stark, C. E. \& Yassa, M. A. (2012) Norepinephrine-mediated emotional arousal facilitates subsequent pattern separation. Neurobiology of Learning and Memory 97(4):465-69. [aMM]

Selden, N. R. W., Cole, B. J., Everitt, B. J. \& Robbins, T. W. (1990) Damage to ceruleo-cortical noradrenergic projections impairs locally cued but enhances spatially cued water maze acquisition. Behavioural Brain Research 39(1):29-51. Available at: http://dx.doi.org/10.1016/0166-4328(90)90119-Y. [RG]

Serences, J. T. \& Yantis, S. (2007) Spatially selective representations of voluntary and stimulus-driven attentional priority in human occipital, parietal, and frontal cortex. Cerebral Cortex 17(2):284-93. [aMM]

Servan-Schreiber, D., Printz, H. \& Cohen, J. D. (1990) A network model of catecholamine effects: Gain, signal-to-noise ratio, and behavior. Science 249 (4971):892-95. [EE, CMW]

Seth, A. K. (2013) Interoceptive inference, emotion, and the embodied self. Trends in Cognitive Sciences 17:565-73. doi: 10.1016/j.tics.2013.09.007. [FF-S]

Shackman, A. J., Salomons, T. V., Slagter, H. A., Fox, A. S., Winter, J. J. \& Davidson, R. J. (2011) The integration of negative affect, pain and cognitive control in the cingulate cortex. Nature Reviews Neuroscience 12(3):154-67. [aMM]

Shakhawat, A. M., Gheidi, A., MacIntyre, I. T., Walsh, M. L., Harley, C. W. \& Yuan, Q. (2015) Arc-expressing neuronal ensembles supporting pattern separation require adrenergic activity in anterior piriform cortex: An exploration of neural constraints on learning. The Journal of Neuroscience 35(41):14070-75. [rMM]

Sheppes, G., Scheibe, S., Suri, G., Radu, P., Blechert, J. \& Gross, J. J. (2014) Emotion regulation choice: A conceptual framework and supporting evidence. Journal of Experimental Psychology. General 143(1):163-81. [TDH]

Sherman, S. M. (2005) Thalamic relays and cortical functioning. Progress in Brain Research 149:107-26. [aMM]

Sheth, S. A., Mian, M. K., Patel, S. R., Asaad, W. F., Williams, Z. M., Dougherty, D D., Bush, G. \& Eskandar, E. N. (2012) Human dorsal anterior cingulate cortex neurons mediate ongoing behavioural adaptation. Nature 488(7410):218-21. [aMM]

Shibata, E., Sasaki, M., Tohyama, K., Kanbara, Y, Otsuka, K., Ehara, S. \& Sakai, A. (2006) Age-related changes in locus ceruleus on neuromelanin magnetic resonance imaging at 3 Tesla. Magnetic Resonance in Medical Sciences 5:197200. [MRN]

Shipp, S. (2003) The functional logic of cortico-pulvinar connections. Philosophical Transactions: Biological Sciences 358(1438):1605-24. doi: 10.2307/3558264. [aMM]

Shohamy, D. \& Adcock, R. A. (2010) Dopamine and adaptive memory. Trends in Cognitive Sciences 14(10):464-72. [MR]

Shumikhina, S. \& Molotchnikoff, S. (1999) Pulvinar participates in synchronizing neural assemblies in the visual cortex, in cats. Neuroscience Letters 272(2):13539. $[\mathrm{aMM}]$

Sidlauskaite, J., Wiersema, J. R., Roeyers, H., Krebs, R. M., Vassena, E., Fias, W., Brass, M., Achten, E. \& Sonuga-Barke, E. (2014) Anticipatory processes in brain state switching - Evidence from a novel cued-switching task implicating default mode and salience networks. NeuroImage 98:359-65. [aMM]

Simon, H. A. (1967) Motivational and emotional controls of cognition. Psychological Review 71(1):29-39. [TDH]

Singer, B. \& Toates, F. M. (1987) Sexual motivation. Journal of Sex Research 23 (4):481-501. [HM]

Singer, W. (1993) Synchronization of cortical activity and its putative role in information processing and learning. Annual Review of Physiology 55(1):349-74. doi: 10.1146/annurev.ph.55.030193.002025. [aMM]

Skelly, L. R. \& Decety, J. (2012) Passive and motivated perception of emotional faces: Qualitative and quantitative changes in the face processing network. PLoS ONE 7(6):e40371. doi: 10.1371/journal.pone.0040371. [aMM]

Sladek, J. R., Jr. \& Sladek, C. D. (1978) Relative quantitation of monoamine histofluorescence in young and old non-human primates. In: Parkinson's disease - II Aging and neuroendocrine relationships, ed. C. E. Finch, D. Potter \& A. D. Kenny, pp. 231-39. Springer. [aMM]

Small, K. M., Brown, K. M., Forbes, S. L. \& Liggett, S. B. (2001) Polymorphic deletion of three intracellular acidic residues of the alpha $2 \mathrm{~B}$-adrenergic receptor decreases $\mathrm{G}$ protein-coupled receptor kinase-mediated phosphorylation and desensitization. The Journal of Biological Chemistry 276:4917-22. [RMT]

Smith, S. D., Most, S. B., Newsome, L. A. \& Zald, D. H. (2006) An emotion-induced attentional blink elicited by aversively conditioned stimuli. Emotion 6(3):52327. [aMM]

Smith, S. L., Smith, I. T., Branco, T. \& Häusser, M. (2013) Dendritic spikes enhance stimulus selectivity in cortical neurons in vivo. Nature 503(7474):115-20. [MEL] 
References/Mather et al.: Norepinephrine ignites local hotspots of neuronal excitation

Snow, P. J., Andre, P. \& Pompeiano, O. (1999) Effects of locus coeruleus stimulation on the responses of SI neurons of the rat to controlled natural and electrical stimulation of the skin. Archives Italiennes de Biologie 137:1-28. [QG]

Sohal, V. S., Zhang, F., Yizhar, O. \& Deisseroth, K. (2009) Parvalbumin neurons and gamma rhythms enhance cortical circuit performance. Nature 459(7247):698702. [aMM]

Soltani, A. \& Koch, C. (2010) Visual saliency computations: Mechanisms, constraints, and the effect of feedback. The Journal of Neuroscience 30(38):12831-43. [aMM]

Sorg, O. \& Magistretti, P. J. (1991) Characterization of the glycogenolysis elicited by vasoactive intestinal peptide, noradrenaline and adenosine in primary cultures of mouse cerebral cortical astrocytes. Brain Research 563(1):227-33. [aMM]

Southwick, S. M., Bremner, J. D., Rasmusson, A., Morgan, C. A. R., Arnsten, A. \& Charney, D. S. (1999) Role of norepinephrine in the pathophysiology and treatment of posttraumatic stress disorder. Biological Psychiatry 46:1192-204. [CGA]

Southwick, S. M., Krystal, J. H., Morgan, C. A., Johnson, D., Nagy, L. M., Nicolaou, A., Heninger, G. R. \& Charney, D. S. (1993) Abnormal noradrenergic function in posttraumatic stress disorder. Archives of General Psychiatry 50:266-74. [CGA]

Srinivasan, N. \& Hanif, A. (2010) Global-happy and local-sad: Perceptual processing affects emotion identification. Cognition and Emotion 24:1062-69. [RHP]

Starke, K. (2001). Presynaptic autoreceptors in the third decade: Focus on $\alpha_{2}$ adrenoceptors. Journal of Neurochemistry 78(4):685-93. [aMM]

Steblay, N. M. (1992) A metaanalytic review of the weapon focus effect. Law and Human Behavior 16(4):413-24. doi: 10.1007/BF02352267. [aMM]

Stein, M. B., Kerridge, C., Dimsdale, J. E. \& Hoyt, D. B. (2007) Pharmacotherapy to prevent PTSD: Results from a randomized controlled proof-of-concept trial in physically injured patients. Journal of Traumatic Stress 20(6):923-32. [rMM]

Steriade, M. \& McCarley, R. W. (2005) Brain control of wakefulness and sleep. Kluwer Academic/Plenum. $\quad[\mathrm{AB}]$

Sterley, T. L., Howells, F. M. \& Russell, V. A. (2013) Maternal separation increases $\mathrm{GABA}(\mathrm{A})$ receptor-mediated modulation of norepinephrine release in the hippocampus of a rat model of ADHD, the spontaneously hypertensive rat. Brain Research 1497:23-31. doi: 10.1016/j.brainres.2012.12.029. [RG]

Sterpenich, V., D’Argembeau, A., Desseilles, M., Balteau, E., Albouy, G., Vandewalle, G., Degueldre, C., Luxen, A., Collette, F. \& Maquet, P. (2006) The locus ceruleus is involved in the successful retrieval of emotional memories in humans. The Journal of Neuroscience 26(28):7416-23. Available at: http://doi. org/10.1523/JNEUROSCI.1001-06.2006. [aMM, SB]

Stins, J. F. \& Beek, P. J. (2007) Effects of affective picture viewing on postural control. BMC Neuroscience 8(1):83. [HM]

Storbeck, J. \& Clore, G. L. (2008) Affective arousal as information: How affective arousal influences judgments, learning, and memory. Social and Personality Psychology Compass 2(5):1824-43. [C-CC, JRH]

Strange, B. A. \& Dolan, R. J. (2004) Beta-adrenergic modulation of emotional memory-evoked human amygdala and hippocampal responses. Proceedings of the National Academy of Sciences of the United States of America 101 (31):11454-58. [aMM, BR, BAS]

Strange, B. A. \& Dolan, R. J. (2007) Beta-adrenergic modulation of oddball responses in humans. Behavioral and Brain Functions 3:29. [aMM]

Strange, B. A., Hurlemann, R. \& Dolan, R. J. (2003) An emotion-induced retrograde amnesia in humans is amygdala- and beta-adrenergic-dependent. Proceedings of the National Academy of Sciences of the United States of America 100 (23):13626-31. Available at: http://www.ncbi.nlm.nih.gov/pubmed/ 14595032. [aMM, RH, BR, BAS, RMT]

Straube, T., Korz, V., Balschun, D. \& Frey, J. U. (2003) Requirement of betaadrenergic receptor activation and protein synthesis for LTP-reinforcement by novelty in rat dentate gyrus. The Journal of Physiology 552(3):953-60. doi: 10.1113/jphysiol.2003.049452. [aMM]

Sutherland, M. R., Lee, T. H. \& Mather, M. (under review) Arousal impairs topdown prioritization in selective attention. [arMM]

Sutherland, M. R. \& Mather, M. (2012) Negative arousal amplifies the effects of saliency in short-term memory. Emotion 12:1367-72. doi: 10.1037/a0027860. [arMM, AM]

Sutherland, M. R. \& Mather, M. (under review) Both positive and negative arousing sounds increase the impact of visual salience. [aMM, RHP]

Sutherland, M. R., McQuiggan, D. A., Ryan, J. D. \& Mather, M. (in press) Perceptual salience does not influence emotional arousal's impairing effects on topdown attention. Emotion $[\mathrm{aMM}]$

Sved, A. F., Cano, G., Passerin, A. M. \& Rabin, B. S. (2002) The locus coeruleus, Barrington's nucleus, and neural circuits of stress. Physiology and Behavior 77 (4):737-42. [aMM]

Svensson, T. H. (1987) Peripheral, autonomic regulation of locus coeruleus noradrenergic neurons in brain: Putative implications for psychiatry and psychopharmacology. Psychopharmacology (Berl) 92:1-7. [HDC]
[HD

Swanson, L. W. \& Hartman, B. K. (1975) The central adrenergic system: An immunofluorescence study of the location of cell bodies and their efferent connections in the rat utilizing dopamine- $B$-hydroxylase as a marker. The
Journal of Comparative Neurology 163(4):467-505. doi: 10.1002 cne.901630406. [aMM]

Takahashi, K., Kayama, Y., Lin, J. S. \& Sakai, K. (2010) Locus coeruleus neuronal activity during the sleep-waking cycle in mice. Neuroscience 169:1116-26. doi 10.1016/j.neuroscience.2010.06.009. [AB]

Talmi, D. (2013) Enhanced emotional memory: Cognitive and neural mechanisms. Current Directions in Psychological Science 22(6):430-36. [aMM]

Tamietto, M. \& de Gelder, B. (2010) Neural bases of the non-conscious perception of emotional signals. Nature Reviews Neuroscience 11(10):697-709. [aMM]
[aM

Tamir, M., Bigman, Y. E., Rhodes, E., Salerno, J. \& Schreier, J. (2015) An expectancy-value model of emotion regulation: Implications for motivation, emotional experience, and decision making. Emotion 15(1):90-103. [TDH]

Tenorio, G., Connor, S. A., Guévremont, D., Abraham, W. C., Williams, J., O’Dell, T. J. \& Nguyen, P. V. (2010) “Silent” priming of translation-dependent LTP by $\beta$-adrenergic receptors involves phosphorylation and recruitment of AMPA receptors. Learning and Memory 17(12):627-38. [aMM]

Terakado, M. (2014) Adrenergic regulation of GABA release from presynaptic terminals in rat cerebral cortex. Cortex 56:49-57. [aMM]

Terburg, D., Morgan, B. E., Montoya, E. R., Hooge, I. T., Thornton, H. B., Hariri, A. R. Panksepp, J., Stein, D. J. \& van Honk, J. (2012) Hypervigilance for fear after basolateral amygdala damage in humans. Translational Psychiatry 2: el15. [BR]

Todd, R. M., Cunningham, W. A., Anderson, A. K. \& Thompson, E. (2012) Affectbiased attention as emotion regulation. Trends in Cognitive Sciences 16:36572. [RMT $]$

Todd, R. M., Ehlers, M. R., Muller, D. J., Robertson, A., Palombo, D. J., Freeman, N., Levine, B. \& Anderson, A. K. (2015) Neurogenetic variations in norepinephrine availability enhance perceptual vividness. The Journal of Neuroscience 35:6506-16. [RMT]

Todd, R. M., Müller, D. J., Lee, D. H., Robertson, A., Eaton, T., Freeman, N., Palombo, D. J., Levine, B. \& Anderson, A. K. (2013) Genes for emotionenhanced remembering are linked to enhanced perceiving. Psychological Science 24(11):2244-53. doi: 10.1177/0956797613492423. [aMM, RMT]

Todd, R. M., Muller, D. J., Palombo, D. J., Robertson, A., Eaton, T., Freeman, N., Levine, B. \& Anderson, A. K. (2014) Deletion variant in the ADRA2B gene increases coupling between emotional responses at encoding and later retrieval of emotional memories. Neurobiological Learning and Memory 112:222-29. [RMT]

Todd, R. M., Palombo, D. J., Levine, B. \& Anderson, A. K. (2011) Genetic differences in emotionally enhanced memory. Neuropsychologia 49(4):734-44. [aMM]

Tooby, J. \& Cosmides, L. (1990) The past explains the present: Emotional adaptations and the structure of ancestral environments. Ethology and Sociobiology 11 (4):375-424. [TDH]

Tooley, V., Brigham, J. C., Maass, A. \& Bothwell, R. K. (1987) Facial recognition: Weapon effect and attentional focus. Journal of Applied Social Psychology 17 (10):845-59. [aMM]

Tose, R., Kushikata, T., Yoshida, H., Kudo, M., Furukawa, K., Ueno, S. \& Hirota, K (2009) Interaction between orexinergic neurons and NMDA receptors in the control of locus coeruleus-cerebrocortical noradrenergic activity of the rat. Brain Research 1250:81-87. [aMM]

Toussay, X., Basu, K., Lacoste, B. \& Hamel, E. (2013) Locus coeruleus stimulation recruits a broad cortical neuronal network and increases cortical perfusion. The Journal of Neuroscience 33(8):3390-401. doi: 10.1523/jneurosci.334612.2013. [aMM]

Tranel, D. \& Damasio, H. (1989) Intact electrodermal skin conductance responses after bilateral amygdala damage. Neuropsychologia 27(4):381-90. Available at http://dx.doi.org/10.1016/0028-3932(89)90046-8. [rMM]

Traynelis, S. F., Wollmuth, L. P., McBain, C. J., Menniti, F. S., Vance, K. M., Ogden, K. K., Hansen, K. B., Yuan, H., Myers, S. J. \& Dingledine, R. (2010) Glutamate receptor ion channels: Structure, regulation, and function. Pharmacological Reviews 62(3):405-96. [aMM]

Treisman, A. (1998) Feature binding, attention and object perception. Philosophical Transactions of the Royal Society of London Series B Biological Sciences 353 (1373):1295-306. [aMM]

Treves, A. \& Rolls, E. T. (1992) Computational constraints suggest the need for two distinct input systems to the hippocampal CA3 network. Hippocampus 2:18999. $[\mathrm{CH}]$

Treviño, M., Frey, S. \& Köhr, G. (2012a) Alpha-1 adrenergic receptors gate rapid orientation-specific reduction in visual discrimination. Cerebral Cortex 22 (11):2529-41. doi: 10.1093/cercor/bhr333. [aMM]

Treviño, M., Huang, S., He, K., Ardiles, A., De Pasquale, R., Guo, Y., Palacios, A., Huganir, R. L. \& Kirkwood, A. (2012b) Pull-push neuromodulation of LTP and LTD enables bidirectional experience-induced synaptic scaling in visual cortex. Neuron 73(3):497-510. [aMM]

Troiani, V. \& Schultz, R. T. (2013) Amygdala, pulvinar \& inferior parietal cortex contribute to early processing of faces without awareness. Frontiers in Human Neuroscience 7:241. doi: 10.3389/fnhum.2013.00241. [aMM] 
Tsuchiya, N., Moradi, F., Felsen, C., Yamazaki, M. \& Adolphs, R. (2009) Intact rapid detection of fearful faces in the absence of the amygdala. Nature Neuroscience 12(10):1224-25. [rMM]

Tully, K. \& Bolshakov, V. Y. (2010) Emotional enhancement of memory: How norepinephrine enables synaptic plasticity. Molecular Brain 3(1):15. [aMM]

Uddin, L. Q. (2015) Salience processing and insular cortical function and dysfunction. Nature Reviews Neuroscience 16(1):55-61. [aMM]

Ueda, H., Goshima, Y., Kubo, T. \& Misu, Y. (1985) Involvement of epinephrine in the presynaptic beta adrenoceptor mechanism of norepinephrine release from rat hypothalamic slices. Journal of Pharmacology and Experimental Therapeutics 232(2):507-12. [aMM]

Ul Haq, R., Liotta, A., Kovacs, R., Rösler, A., Jarosch, M. J., Heinemann, U. \& Behrens, C. J. (2012) Adrenergic modulation of sharp wave-ripple activity in rat hippocampal slices. Hippocampus 22(3):516-33. doi: 10.1002/hipo.20918. [aMM]

Ullsperger, M., Harsay, H., Wessel, J. \& Ridderinkhof, K. R. (2010) Conscious perception of errors and its relation to the anterior insula. Brain Structure and Function 214(5/6):629-43. doi: 10.1007/s00429-010-0261-1. [arMM]

U'Prichard, D. C., Bechtel, W. D., Rouot, B. M. \& Snyder, S. H. (1979) Multiple apparent alpha-noradrenergic receptor binding sites in rat brain: Effect of 6hydroxydopamine. Molecular Pharmacology 16:47-60. [RMT]

Usher, M., Cohen, J. D., Servan-Schreiber, D., Rajkowski, J. \& Aston-Jones, G. (1999) The role of locus coeruleus in the regulation of cognitive performance. Science 283(5401):549-54. [aMM, EE, CMW]

Valentino, R. J. \& Van Bockstaele, E. (2001) Opposing regulation of the locus coeruleus by corticotropin-releasing factor and opioids. Psychopharmacology 158 (4):331-42. [aMM]

Van Bockstaele, E., Bajic, D., Proudfit, H. \& Valentino, R. (2001) Topographic architecture of stress-related pathways targeting the noradrenergic locus coeruleus. Physiology and Behavior 73(3):273-83. [aMM]

Van Bockstaele, E. J., Colago, E. E. O. \& Valentino, R. J. (1998) Amygdaloid corticotropin-releasing factor targets locus coeruleus dendrites: Substrate for the co-ordination of emotional and cognitive limbs of the stress response. Journal of Neuroendocrinology 10(10):743-58. doi: 10.1046/j.1365-2826.1998.00254.x. $[\mathrm{aMM}]$

Van de Cruys, S. \& Wagemans, J. (2011) Putting reward in art: A tentative prediction error account of visual art. $i$-Perception 2(9):1035-62. [C-CC]

Van Dillen, L. F., Papies, E. K. \& Hofmann, W. (2013) Turning a blind eye to temptation: How cognitive load can facilitate self-regulation. Journal of Personality and Social Psychology 104(3):427-43. [TDH]

Van Horn, M. R., Sild, M. \& Ruthazer, E. S. (2013) D-Serine as a gliotransmitter and its roles in brain development and disease. Frontiers in Cellular Neuroscience 7:39. doi: 10.3389/fncel.2013.00039. [aMM]

van Stegeren, A. H., Roozendaal, B., Kindt, M., Wolf, O. T. \& Joels, M. (2010) Interacting noradrenergic and corticosteroid systems shift human brain activation patterns during encoding. Neurobiology of Learning and Memory 93(1):56-65. Available at: http://www.ncbi.nlm.nih.gov/pubmed/19695335. [RH]

Vankov, A., Hervé-Minvielle, A. \& Sara, S. J. (1995) Response to novelty and its rapid habituation in locus coeruleus neurons of the freely exploring rat. European Journal of Neuroscience 7(6):1180-87. doi: 10.1111/j.1460-9568.1995.tb01108.x. [aMM, RG, SJS]

Varazzani, C., San-Galli, A., Gilardeau, S. \& Bouret, S. (2015) Noradrenaline and dopamine neurons in the reward/effort trade-off: A direct electrophysiological comparison in behaving monkeys. The Journal of Neuroscience 35(20):7866-77. Available at: http://doi.org/10.1523/JNEUROSCI.0454-15.2015. [SB]

Varga, C., Oijala, M., Lish, J., Szabo, G. G., Bezaire, M., Marchionni, I., Golshani, P. \& Soltesz, I. (2014) Functional fission of parvalbumin interneuron classes during fast network events. eLife 3:e04006. doi: 10.7554/eLife.04006. [aMM]

Vazey, E. M. \& Aston-Jones, G. (2014) Designer receptor manipulations reveal a role of the locus coeruleus noradrenergic system in isoflurane general anesthesia. Proceedings of the National Academy of Sciences of the United States of America $111(10): 3859-64 . \quad$ [aMM]

Venkatesan, C., Song, X. Z., Go, C. G., Kurose, H. \& Aoki, C. (1996) Cellular and subcellular distribution of $\alpha_{2 \mathrm{~A}}$-adrenergic receptors in the visual cortex of neonatal and adult rats. Journal of Comparative Neurology 365(1):79-95. $[\mathrm{rMM}]$

Vezzani, A., Wu, H. Q. \& Samanin, R. (1987) $\left[{ }^{3} \mathrm{H}\right]$ Norepinephrine release from hippocampal slices is an in vitro biochemical tool for investigating the pharmacological properties of excitatory amino acid receptors. Journal of Neurochemistry 49(5):1438-42. [aMM]

Videen, T. O., Daw, N. W. \& Rader, R. K. (1984) The effect of norepinephrine on visual cortical neurons in kitten and adult cats. Journal of Neuroscience 4:160717. $[\mathrm{QG}]$

Vijayashankar, N. \& Brody, H. (1979) Quantitative study of the pigmented neurons in the nuclei locus coeruleus and subcoeruleus in man as related to aging. Journal of Neuropathology and Experimental Neurology 38(5):490-97. doi: 10.1097/00005072-197909000-00004. [aMM]
Vinck, M., Batista-Brito, R., Knoblich, U. \& Cardin, J. A. (2014) Arousal and locomotion make distinct contributions to cortical activity patterns and visual encoding. Neuron 86(3):740-754. [aMM]

Viola, H., Ballarini, F., Martínez, M. C. \& Moncada, D. (2014) The tagging and capture hypothesis from synapse to memory. In: Progress in molecular biology and translational science, vol. 122, ed. Z. U. Khan and E. Chris Muly, pp. $391-$ 423. Academic Press. [MR]

Vizi, E., Fekete, A., Karoly, R. \& Mike, A. (2010) Non-synaptic receptors and transporters involved in brain functions and targets of drug treatment. British Journal of Pharmacology 160(4):785-809. [aMM]

Vogel, B. O., Shen, C. \& Neuhaus, A. H. (2015a) Emotional context facilitates cortical prediction error responses. Human Brain Mapping 36(9):3641-52. doi: 10.1002/hbm.22868. [FF-S, rMM ]

Vogel, S., Klumpers, F., Krugers, H. J., Fang, Z., Oplaat, K. T., Oitzl, M. S., Joels, M $\&$ Fernandez, G. (2015b) Blocking the mineralocorticoid receptor in humans prevents the stress-induced enhancement of centromedial amygdala connectivity with the dorsal striatum. Neuropsychopharmacology 40(4):947-56. Available at: http://www.ncbi.nlm.nih.gov/pubmed/25355243. [RH]

Von der Gablentz, J., Tempelmann, C., Münte, T. \& Heldmann, M. (2015) Performance monitoring and behavioral adaptation during task switching: An fMRI study. Neuroscience 285:227-35. [aMM, SJS]

Von Stein, A. \& Sarnthein, J. (2000) Different frequencies for different scales of cortical integration: From local gamma to long range alpha/theta synchronization. International Journal of Psychophysiology 38(3):301-13. [aMM]

Vuilleumier, P. (2005a) Emotion and attention interactions in social cognition: Brain regions involved in processing anger prosody. NeuroImage 28:848-58. doi: 10.1016/j.neuroimage.2005.06.023. [AM]

Vuilleumier, P. (2005b) How brains beware: Neural mechanisms of emotional attention. Trends in Cognitive Sciences 9(12):585-94. [aMM]

Vuilleumier, P., Richardson, M. P., Armony, J. L., Driver, J. \& Dolan, R. J. (2004) Distant influences of amygdala lesion on visual cortical activation during emotional face processing. Nature Neuroscience 7(11):1271-78. [BR]

Walling, S. G., Brown, R. A. M., Milway, J. S., Earle, A. G. \& Harley, C. W. (2011) Selective tuning of hippocampal oscillations by phasic locus coeruleus activation in awake male rats. Hippocampus 21(11):1250-62. doi: 10.1002/hipo.20816. [arMM]

Walls, A. B., Heimbürger, C. M., Bouman, S. D., Schousboe, A. \& Waagepetersen, H. S. (2009) Robust glycogen shunt activity in astrocytes: Effects of glutamatergic and adrenergic agents. Neuroscience 158(1):284-92. [aMM]

Wang, J. K., Andrews, H. \& Thukral, V. (1992) Presynaptic glutamate receptors regulate noradrenaline release from isolated nerve terminals. Journal of $\mathrm{Neu}$ rochemistry 58(1):204-11. [aMM]

Wang, L., Kennedy, B. L. \& Most, S. B. (2012) When emotion blinds: A spatiotemporal competition account of emotion-induced blindness. Frontiers in Psychology 3:438. doi: 10.3389/fpsyg.2012.00438. [aMM]

Wang, M., Ramos, B., Paspalas, C., Shu, Y., Simen, A., Duque, A., Vijayraghavan, S., Brennan, A., Dudley, A., Nou, E., Mazer, J. A., McCormick, D. A. \& Arnsten, A. F. T. (2007) Alpha2A-adrenoceptor stimulation strengthens working memory networks by inhibiting cAMP-HCN channel signaling in prefrontal cortex. Cell 129:397-410. [aMM, CGA]

Wang, M., Yang, Y., Wang, C. J., Gamo, N. J., Jin, L. E., Mazer, J. A., Morrison, J. H. Wang, X. J. \& Arnsten, A. F. (2013) NMDA receptors subserve working memory persistent neuronal firing in dorsolateral prefrontal cortex. Neuron 77 (4):736-49. [CGA]

Wang, S.-H., Redondo, R. L. \& Morris, R. G. M. (2010) Relevance of synaptic tagging and capture to the persistence of long-term potentiation and everyday spatial memory. Proceedings of the National Academy of Sciences of the United States of America 107(45):19537-42. [MR]

Wang, X. J. (2002) Probabilistic decision making by slow reverberation in cortical circuits. Neuron 36(5):955-68. [CMW]

Wang, Z. \& McCormick, D. A. (1993) Control of firing mode of corticotectal and corticopontine layer $\mathrm{V}$ burst-generating neurons by norepinephrine, acetylcholine, and 1S, 3R-ACPD. The Journal of Neuroscience 13(5):2199-216. [aMM, MEL]

Waring, J. D. \& Kensinger, E. A. (2011) How emotion leads to selective memory: Neuroimaging evidence. Neuropsychologia 49(7):1831-42. [aMM]

Waterhouse, B. D., Azizi, S. A., Burne, R. A. \& Woodward, D. J. (1990) Modulation of rat cortical area 17 neuronal responses to moving visual stimuli during norepinephrine and serotonin microiontophoresis. Brain Research 514:276-92. [RN]

Waterhouse, B. D., Devilbiss, D., Fleischer, D., Sessler, F. M. \& Simpson, K. L. (1998a) New perspectives on the functional organization and postsynaptic influences of the locus ceruleus efferent projection system. Advances in Pharmacology and Chemotherapy 42:749-54. [QG]

Waterhouse, B. D., Moises, H. C. \& Woodward, D. J. (1981) Alpha-receptormediated facilitation of somatosensory cortical neuronal responses to excitatory synaptic inputs and iontophoretically applied acetylcholine. Neuropharmacology 20:907-20. [CGA] 
References/Mather et al.: Norepinephrine ignites local hotspots of neuronal excitation

Waterhouse, B. D., Moises, H. C. \& Woodward, D. J. (1998b) Phasic activation of the locus coeruleus enhances responses of primary sensory cortical neurons to peripheral receptive field stimulation. Brain Research 790:33-44. [QG]

Waterhouse, B. D., Moises, H. C., Yeh, H. H. \& Woodward, D. J. (1982) Norepinephrine enhancement of inhibitory synaptic mechanisms in cerebellum and cerebral cortex: Mediation by beta adrenergic receptors. Journal of Pharmacology and Experimental Therapeutics 221:495-506. [RN]

Waterhouse, B. D., Mouradian, R., Sessler, F. M. \& Lin, R. C. (2000) Differential modulatory effects of norepinephrine on synaptically driven responses of layer $\mathrm{V}$ barrel field cortical neurons. Brain Research 868:39-47. [CGA]

Waterhouse, B. D., Sessler, F. M., Cheng, J. T., Woodward, D. J., Azizi, S. A. \& Moises, H. C. (1988) New evidence for a gating action of norepinephrine in central neuronal circuits of mammalian brain. Brain Research Bulletin 21:425-32. [RN]

Waterhouse, B. D. \& Woodward, D. J. (1980) Interaction of norepinephrine with cerebrocortical activity evoked by stimulation of somatosensory afferent pathways in the rat. Experimental Neurology 67(1):11-34. Available at: http://dx.doi. org/10.1016/0014-4886(80)90159-4. [aMM, EE]

Weierich, M. R., Wright, C. I., Negreira, A., Dickerson, B. C. \& Barrett, L. F. (2010) Novelty as a dimension in the affective brain. NeuroImage 49(3):2871-78. [aMM]

Weinbach, N. \& Henik, A. (2012) The relationship between alertness and executive control. Journal of Experimental Psychology: Human Perception and Performance 38:1530-40. doi: 10.1037/a0027875. [CMW]

Weinbach, N. \& Henik, A. (2014) Alerting enhances attentional bias for salient stimuli: Evidence from a global/local processing task. Cognition 133(2):414-19. [CMW]

White, C. N., Ratcliff, R. \& Starns, J. J. (2011) Diffusion models of the flanker task: Discrete versus gradual attentional selection. Cognitive Psychology 63:210-38. doi: 10.1016/j.cogpsych.2011.08.001. [CMW]

Wieser, M. J., McTeague, L. M. \& Keil, A. (2011) Sustained preferential processing of social threat cues: Bias without competition? Journal of Cognitive Neuroscience 23(8):1973-86. [aMM]

Wilhelm, H. (2008) The pupil. Current Opinion in Neurology 21(1):36-42. [rMM]

Williams, J. M. G., Mathews, A. \& MacLeod, C. (1996) The emotional Stroop task and psychopathology. Psychological Bulletin 120(1):3-24. [HM]

Wilson, R. C., Geana, A., White, J. M., Ludvig, E. A. \& Cohen, J. D. (2014) Humans use directed and random exploration to solve the explore-exploit dilemma. Journal of Experimental Psychology: General 143:2074-81. [MRN]

Wilson, R. S., Nag, S., Boyle, P. A., Hizel, L. P., Yu, L., Buchman, A. S., Schneider, J. A. \& Bennett, D. A. (2013) Neural reserve, neuronal density in the locus ceruleus, and cognitive decline. Neurology 80(13):1202-208. [aMM, MRN]

Winkielman, P. \& Cacioppo, J. T. (2001) Mind at ease puts a smile on the face: Psychophysiological evidence that processing facilitation elicits positive affect. Journal of Personality and Social Psychology 81(6):989-1000. [rMM]
Wolosker, H. (2007) NMDA receptor regulation by D-serine: New findings and perspectives. Molecular Neurobiology 36(2):152-64. [aMM]

Wulff, P., Ponomarenko, A. A., Bartos, M., Korotkova, T. M., Fuchs, E. C., Bähner, F., Both, M., Tort, A. B. L., Kopell, N. J., Wisden, W. \& Monyer, H. (2009) Hippocampal theta rhythm and its coupling with gamma oscillations require fast inhibition onto parvalbumin-positive interneurons. Proceedings of the National Academy of Sciences of the United States of America 106(9):3561-66. doi: 10.1073/pnas.0813176106. [aMM]

Xu, N. L., Harnett, M. T., Williams, S. R., Huber, D., O’Connor, D. H., Svoboda, K. \& Magee, J. C. (2012) Nonlinear dendritic integration of sensory and motor input during an active sensing task. Nature 492(7428):247-51. [MEL]

Xue, M., Atallah, B. V. \& Scanziani, M. (2014) Equalizing excitation-inhibition ratios across visual cortical neurons. Nature 511(7511):596-600. [aMM]

Yellin, D., Berkovich-Ohana, A. \& Malach, R. (2015) Coupling between pupil fluctuations and resting-state fMRI uncovers a slow build-up of antagonistic responses in the human cortex. NeuroImage 106:414-27. [aMM]

Yoss, R. E., Moyer, N. J. \& Hollenhorst, R. W. (1970) Pupil size and spontaneous pupillary waves associated with alertness, drowsiness, and sleep. Neurology 20 (6):545-54. [rMM]

Yu, A. J. \& Dayan, P. (2005) Uncertainty, neuromodulation, and attention. Neuron 46(4):681-92. Available at: http://doi.org/10.1016/j.neuron.2005.04.026. [arMM, SB]

Yue, B. W. \& Huguenard, J. R. (2001) The role of H-current in regulating strength and frequency of thalamic network oscillations. Thalamus and Related Systems $1(02): 95-103 . \quad[\mathrm{aMM}]$

Zhou, Y., Won, J., Karlsson, M. G., Zhou, M., Rogerson, T., Balaji, J., Neve, R., Poirazi, P. \& Silva, A. J. (2009) CREB regulates excitability and the allocation of memory to subsets of neurons in the amygdala. Nature Neuroscience 12 (11):1438-43. [aMM]

Zhu, H., Brodsky, M., Gorman, A. L. \& Inturrisi, C. E. (2003) Region-specific changes in NMDA receptor mRNA induced by chronic morphine treatment are prevented by the co-administration of the competitive NMDA receptor antagonist LY274614. Molecular Brain Research 114(2):154-62. [aMM]

Zilles, K. \& Amunts, K. (2009) Receptor mapping: Architecture of the human cerebral cortex. Current Opinion in Neurology 22(4):331-39. [aMM]

Zillmann, D. (1983) Transfer of excitation in emotional behavior. In: Social psychophysiology: A sourcebook, ed. J. T. Cacioppo \& R. E. Petty, pp. 215-40. Guilford Press. [KK]

Zitnik, G. A., Clark, B. D. \& Waterhouse, B. D. (2014) Effects of intracerebroventricular corticotropin releasing factor on sensory-evoked responses in the rat visual thalamus. Brain Research 1561:35-47. Available at: http://dx.doi.org/10. 1016/j.brainres.2014.02.048. [aMM] 